\title{
On Autonomous and Teleoperated Aerial Service Robots
}

\author{
Theory and Application
}

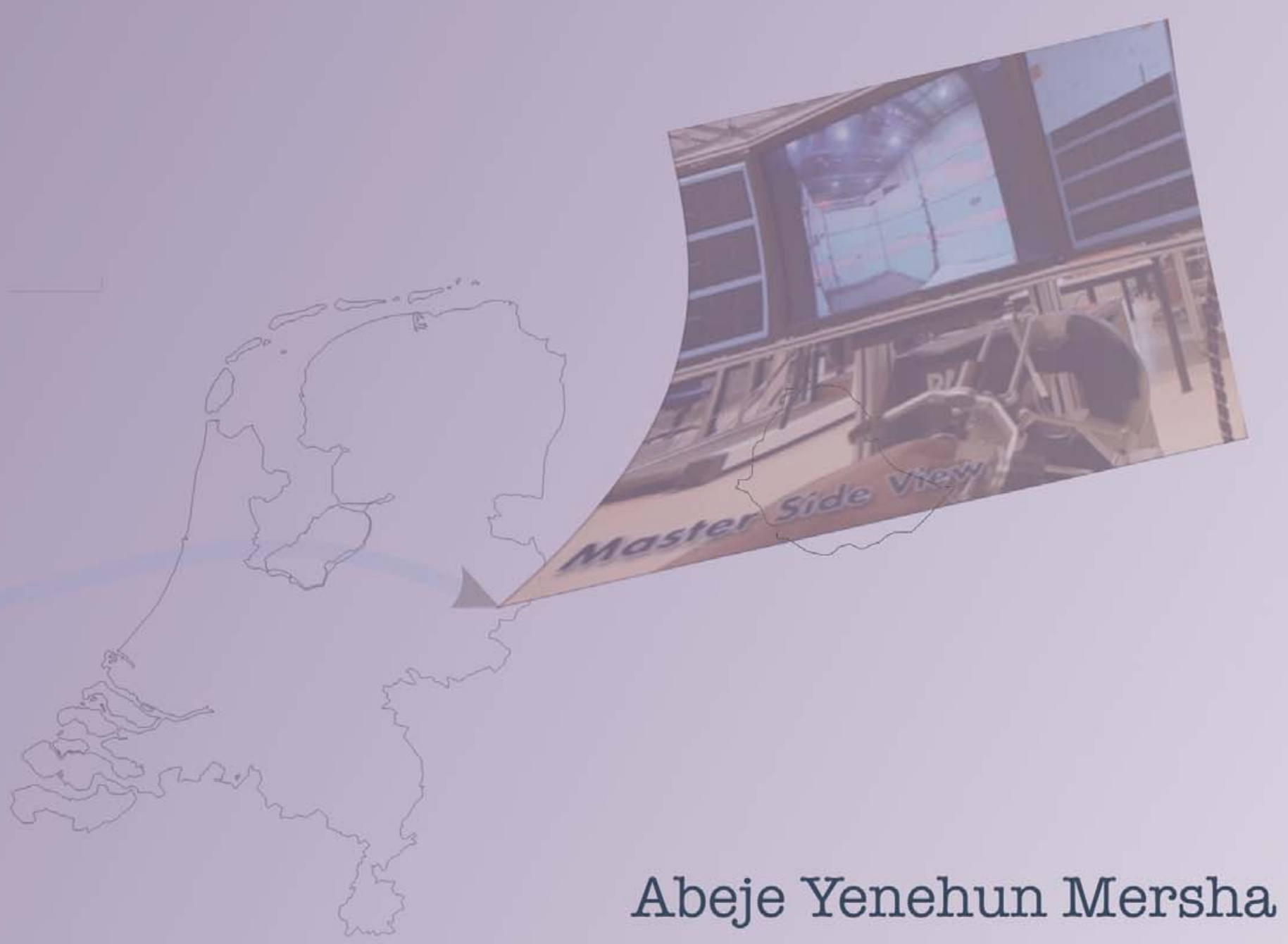




\section{ON AUTONOMOUS AND TELEOPERATED AERIAL SERVICE ROBOTS: \\ THEORY AND APPLICATION}

ABEJE YENEHUN MERSHA 
$\mathrm{PhD}$ dissertation committee:

Chairman and Secretary:

Prof. dr. P.M.G. Apers

Promotor:

Prof. dr. ir. S. Stramigioli University of Twente, NL

Co-promotor:

Dr. R. Carloni

Members:

Prof. dr. R. Mahony

University of Twente, NL

Prof. dr. L. Marconi

Australian National University, Australia

University of Bologna, Italy

Prof. dr. ir. J.M.A. Scherpen

University of Groningen, NL

Prof. dr. ir. M. Steinbuch

Eindhoven University of Technology, NL

$\begin{array}{ll}\text { Prof. dr. ing. P.J.M. Havinga } & \text { University of Twente, NL } \\ \text { Prof. dr. ir. J. van Amerongen } & \text { University of Twente, NL }\end{array}$

The research described in this thesis has been carried out in the Robotics and Mechatronics (RAM) Group, which is a part of the Institute of Centre for Telematics and Information Technology (CTIT) at the University of Twente, and has been financially supported by the European Commission's Seventh Framework Program as part of the AIRobots project under grant no. 248669.
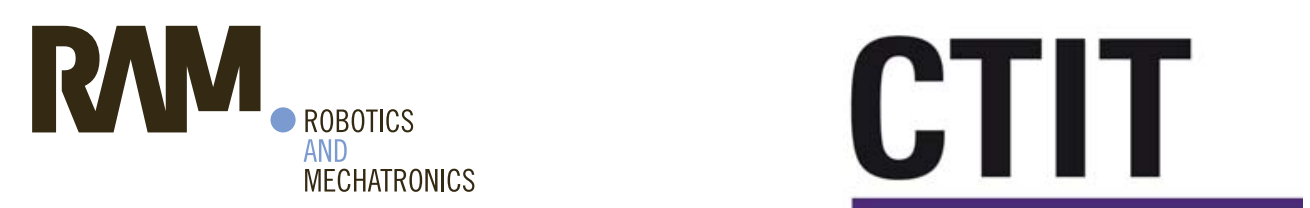

\section{UNIVERSITY OF TWENTE.}

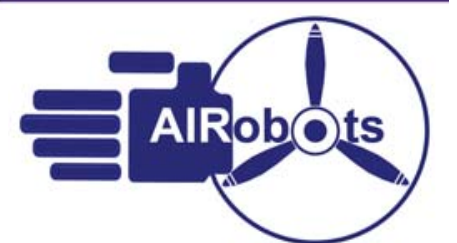

The research reported in this thesis is part of the research program of the Dutch Institute of Systems and Control (DISC). The author has successfully completed the educational program of the Graduate School DISC.

Title: On autonomous and teleoperated aerial service robots: theory and application

Author: Abeje Yenehun Mersha

ISBN: $\quad$ 978-90-365-3658-5

ISSN: $\quad$ 1381-3617 (CTIT Ph.D. Thesis Series No. 14-311)

DOI: $\quad$ 10.3990./1.9789036536585

http://dx.doi.org/10.3990/1.9789036536585

Copyright@ 2014, by Abeje Yenehun Mersha, Enschede, The Netherlands.

All rights reserved. No part of this publication may be reproduced, distributed or transmitted in any form or by any means without the prior written permission of the copyright owner, except in the case of brief quotations for academic reviews. 


\title{
ON AUTONOMOUS AND TELEOPERATED AERIAL SERVICE ROBOTS:

\author{
THEORY AND APPLICATION
}

\author{
DISSERTATION \\ to obtain \\ the degree of doctor at the University of Twente, \\ on the authority of the rector magnificus, \\ prof. dr. H. Brinksma, \\ on account of the decision of the graduation committee, \\ to be publicly defended \\ on Friday 13 June 2014 at 14:45
}

by

Abeje Yenehun Mersha

born on 14 August 1984

in Addis Ababa, Ethiopia. 
This dissertation has been approved by:

Prof. dr. ir. S. Stramigioli, Promotor

Dr. R. Carloni, Co-promotor

Copyright(c) 2014, by Abeje Yenehun Mersha, Enschede, The Netherlands. 
To my most beautiful, precious and priceless princess, my (l|w)ife;- Hiwote 



\title{
ACKNOWLEDGMENT
}

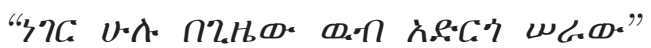 \\ "God has made everything beautiful in its time."
}

a

Ecclesiastes 3:11

It is always quite satisfying to finish a chapter from the book of Life. No matter how bumpy the road may be, it always presents an experience to learn from. This chapter of my scientific journey began during a casual conversation with Raffaella on my Modern Robotics exam, followed by an offer on a possible master's assignment with $\mathrm{PhD}$ position within the AIRobots project.

First, I would like to thank Raffaella for offering me the opportunity to work on this challenging as well as exciting research topic, which have resulted in a fruitful and enjoyable collaboration in the past years. I am grateful for the unrestricted research freedom you have given me during this period. Your continuous and timely feedback on my thesis and all our other scientific works were very instrumental. I would also like to thank my promotor, Stefano, for all the constructive discussions we have had over the years. Despite the fact that our short meetings were very few in number and we often had differences in approach (I believe it is one of the beauties of science), they were all insightful in one way or another, and have broaden my scientific spectrum.

At the beginning of my research, I was the only student working on aerial robotics in our group. But in collaboration with other students and members of the group that came along during this period, we have established ourselves to be one of the few world-class groups active in the field of aerial service robots. In this process, I had the privilege to supervise few students. I am thankful to all of them as they have contributed to this work in some form and various capacities; special thanks to Andi for his great contribution in setting up the initial software architecture and the flight arena.

Matteo, thanks for the wide range of scientific collaborations and friendship we have developed through the years. The countless sleepless nights spent in Bologna and Zürich, during the AIRobots Integration Weeks and Review Meetings, are unforgettable. It is quite remarkable that we had transformed a tiresome day-long drive to Zürich for the AIRobots IW3 to a brainstorming session, during which various points of scientific collaboration were brought up. The workshop we organized at IROS2013 in Tokyo was also conceived during this trip. I will be looking forward to the rest of our collaborations. 
The research in this thesis has been conducted as part of the AIRobots project supported by the European Commission's Seventh Framework Program. Although the project was quite demanding and time consuming, it gave me an opportunity to collaborate with a number of great people; thank-you fellow AIRoboticists. Specially thanks to Lorenzo Marconi, the coordinator of the AIRobots project, for being an inspirational scientist. I am honored to have you in my dissertation committee.

Xiaolie Hou (Eric) and Robert Mahony, thank you for our successful quest to realize the longest intercontinental (Netherlands $\Leftrightarrow$ Australia) haptic teleoperation of aerial robots ever! Looking back, the results we have achieved are worth the countless email exchanges, skype meetings and failed experimental trials, not to mention that Eric had to stay up many nights past midnight and I had to wake up as early as 4 AM to offset the time difference between Canberra and Enschede (). Rob, your critical and constructive comments only added greatly to the value of our collaboration. I am really glad to have you here in my dissertation committee.

I am also thankful to the rest of my dissertation committee: Prof. van Amerongen, Prof. Havinga, Prof. Scherpen and Prof. Steinbuch, for reading my thesis and for being willing to grill me during my public defense. Special thanks to Prof. van Amerongen for your mentorship during my MSc. study.

I am grateful to Jolanda and Carla for all the support during my stay in the group. Our technicians, Gerben, Marcel and Alfred, your technical support is highly appreciated. I would also like to thank all the members of our group for creating conducive and dynamic working environment; I enjoyed our lunches, coffee+'s, and group activities during the yearly brainstorming sessions. I also enjoyed the company of my colleagues during the various international conferences that spanned three continents. Furthermore, special thanks to our group's football team, which I had the honor to have captained. Although you always gave me hard time while trying to assemble a full team, obviously due to other obligations, our winning spirit when we are on the pitch have been amazing.

All my former and current office-mates deserve a special thanks for all the fun times we have spent together through the years. In particular, I am thankful to: Bayan, Yury, Yunyun, Douwe, Oguzcan, Xiaochen, Bart and Tadele, for the special bond we have developed through our various social activities. The numerous board-game nights, which appears to be another name for couples night $(-)$, have been quite wonderful. The road trip to Budapest for Yury and Edit's wedding was really memorable. I will certainly miss our afternoon-walks when I leave the group.

Besides the academic environment, there are a number of people that made life in Enschede very pleasant. I thank Maaike for all tips on life in the Netherlands, and of course the "gezellig" daguitjes. I am immensely grateful to Johannes and his family for making us feel comfortable during our "weekly" visit to St. Mary church.

Bayan and Bert, thank you for being friends in need. Beside everything else, you are always willing to go extra mile to help. Our numerous social activities filled with endless teasing, and sometimes serious discussions have been enriching. Above all, as Hiwote once said, "Thank you for making us laugh" (-). 
Merry and Mesfine, thank you for being true friends, and making us feel at home away from home. There is no better friend than who surprises with a spontaneous invitation for a lovely home made Ethiopian dish, especially after a long day at work. The addition of Abi to the family was the icing on the cake; her presence always enlightens the room.

Although it is difficult to list down the names of all the people that have touched and shaped my life in one way or another, I am ceaselessly grateful to all: family, friends and teachers back in Lideta Catholic Cathedral School (LCCS) and Mekelle University. Dear Abba Tekle, I am very grateful for your never ending mentorship, blessings and friendship ever since I joined LCCS as a KG student.

Finally and most importantly, I would like to express my profound gratitude to my family from both sides of the aisle. Ababa, Amelework, Emushe and Abnet, your constant encouragement and all-rounded support meant a lot. I am very happy to be part your family! Yenehunye and Motheye, I am eternally grateful to have parents like you. You have provided everything I asked for. You have instilled the importance of family and quality education at early age, and you have taught me to stay true to my values; which shaped who I am as a person today. BTW, you are right to be proud of me -$)$. Daduye, thank-you only wouldn't be fair to express how grateful I am for your effort in bringing me up, no matter how rebellious I was

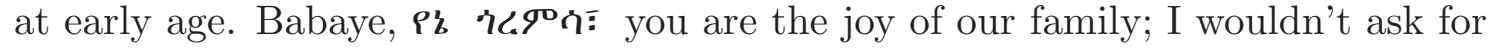

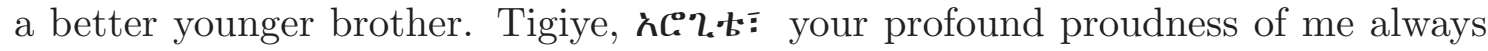
motivates me to make you even prouder. And of course Antiye, my protective brother, you are the rock of our family. You are the best role model I could ever ask for. I really don't know how to express how highly indebted I am to you for everything you have done

Hiwote $\mathbf{P}_{\mathbf{b}} \mathbf{9} \mathbf{q} \mathbf{\Phi} \mathbf{C}_{\mathbf{E}} \mathbf{z}$ it sounds cliché when I say I love you more than you will ever know, but it is very trueeeeee. You are the only person who makes my life worth living. I had the privilege of having smarter wife in every sense of the word. I wish I could redirect some of the questions I will be asked during my defense $\odot$. In particular during this research period, to say that your invaluable input in our countless technical discussions, tips \& tricks, and of course your ruthless critics on my papers made the real difference is an understatement. You are also the only person who makes me pause for a second and doubt my decision. I can't thank God enough for blessing me with you, $\boldsymbol{P}_{\mathbf{b}} \mathbf{\$} \mathbf{3} \boldsymbol{\xi}$. Your never ending and unconditional love keeps me going at all times. Thank you for being the beautiful person that you are. This and all good things in my life are all because of you, and I know,

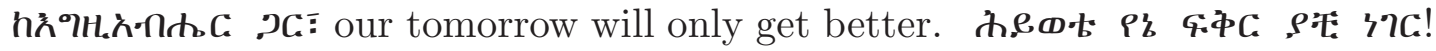

Abeje Yenehun Mersha

June 2014,

Enschede, The Netherlands

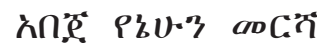

n's 9.90 .

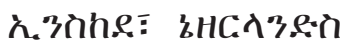





\section{SUMMARY}

Traditionally, aerial robots have been used in applications that do not require physical interaction with the environment. Recently, however, there is a growing interest in using aerial robots for applications that involve active but nondestructive interaction with the environment, especially in the field of service robotics. Aerial service robots are poised to be fundamental parts of tomorrow's service applications for their low-cost, high efficiency, and safety.

The main goal of this thesis is the development of new and advanced control architectures for autonomous and teleoperated aerial service robots. The first part of this thesis describes three different tele-control architectures for aerial robots, whereas the second part describes an autonomous control architecture for aerial manipulators.

The first tele-control architecture addresses both classical challenges, such as time delays and packet losses, and peculiar challenges attributed to using aerial robots, such as pervasive energy dissipation, underactuation, and workspace incompatibility between the master haptic interface and the slave aerial robot. This architecture is designed based on energetic consideration using port-based approach. It works in a plug-and-play fashion with different types of aerial robots and haptic interfaces, and remains passive under various operating conditions.

The second architecture is aimed at achieving improved transparency (both vehicle and environment transparencies), according to a comprehensive definition and measures of transparency provided in this thesis. This controller specifically incorporates methodologies that counter the degradation of transparency due to network-induced imperfections. The significance of these methodologies has been demonstrated by experiments that also include the longest intercontinental haptic teleoperation of aerial robots ever. This architecture is implemented for admittance and impedance tele-control frameworks, providing insight on the different features of both frameworks.

In a step towards achieving human-like capability, the third control architecture aims at varying the impedance of the controlled aerial robot and regulating its interaction force in a unified manner, while providing a multimodal feedback to the operator to achieve transparency. Beyond classical teleoperation that only relies on exchange of position (velocity)/force information to execute the task, this controller also varies the impedance according to the operator's intention. This enables the 
operator to accomplish wide range of tasks, both interactive and noninteractive, in a stable manner while achieving high task-performance. The inclusion of slidingmode observer as part of both the master and the slave controllers adds greatly to the versatility of this control architecture.

Besides the slave controllers designed in the context of teleoperation, which can be used autonomously for various tasks, such as trajectory tracking, obstacle avoidance, and aerial interaction, the last part of this thesis describes a novel autonomous controller for aerial manipulators. This controller is designed based on the unified dynamics of the aerial manipulator, and additionally exploits the dynamics of the robotic manipulator appended to the aerial robot. Results show that this controller greatly improves the stability robustness of the controlled aerial manipulator and expands the flight envelop compared to the current state of the art. 


\section{SAMENVATTING}

Traditioneel worden vliegende robots gebruikt in toepassingen die geen fysieke interactie met de omgeving nodig hebben. Recent is er echter een groeiende interesse in het gebruik van vliegende robots voor applicaties die gebruik maken van actieve, maar niet destructieve, interactie met de omgeving. Dit betreft in het bijzonder het gebied van service robotica. Vliegende service robots kunnen een fundamenteel onderdeel worden van toekomstige service applicaties, vanwege hun lage kostprijs, hoge efficiency en hoge veiligheid.

Het hoofddoel van dit proefschrift is de ontwikkeling van nieuwe en geavanceerde regelarchitecturen voor autonome en op afstand bestuurbare vliegende service robots. Het eerste deel van dit proefschrift beschrijft drie verschillende telebesturingarchitecturen voor vliegende robots. Terwijl het tweede deel een architectuur van autonome regeling voor vliegende manipulatoren beschrijft.

De eerst beschreven telebesturing architectuur richt zich op zowel de klassieke uitdagingen, zoals tijdsvertragingen en verlies van pakketten, als ook de uitzonderlijke uitdagingen bij het gebruik van vliegende robots, zoals de verbreide energie dissipatie, onderactuatie, en incompatibiliteit van het werkgebied van de master haptische interface en de slave vliegende robot. Deze architectuur is ontworpen met energetische afwegingen, gebruikmakend van een poort gebaseerde benadering. Het kan op een plug-and-play manier geïmplementeerd worden met verschillende vliegende robot types en haptische interfaces, en blijft stabiel onder diverse omstandigheden.

De tweede architectuur beoogt een verbeterde transparantie (zowel de voertuig als de omgeving transparatie), gebruikmakend van een uitgebreide definitie en methodes van transparantie beschreven in dit proefschrift. Deze regelaar omvat in het bijzonder methoden die de degradatie van transparantie door netwerk geïnduceerde onvolkomenheden tegenwerken. Het belang van deze methoden is aangetoond met experimenten, waaronder de langste intercontinentale haptische teleoperatie van vliegende robots ooit. Deze architectuur is geïmplementeerd volgens de admittantie en impedantie telebesturing methodiek, hetgeen inzicht in de verschillende functies van beide methodieken verschaft.

Strevend naar een mensachtige bekwaamheid is de derde regelarchitectuur gericht op het variëren van de impedantie van de aangestuurde vliegende robot, in combinatie met het regelen van de interactie kracht op een uniforme wijze, terwijl 
multi-modale terugkoppeling aan de operator gegeven wordt om transparantie te bereiken. Naast de uitwisseling van de positie (snelheid)/kracht informatie om de taak uit te voeren, zoals bij klassieke regelaars, varieeert deze regelaar ook de impelementatie volgens de intentie van de bestuurder. Hierdoor kan de operator een breed scala van taken uitvoeren, zowel interactief als niet-interactief, op een stabiele wijze, gepaard gaand met het bereiken van een hoge prestatie. De integratie van de sliding-modus waarnemer op zowel de master als de slave regelaar, draagt sterk bij aan de veelzijdigheid van deze regelarchitectuur.

Naast de slave regelaars, ontworpen in het kader van teleoperatie, kunnen worden gebruikt voor diverse taken, zoals het volgen van een baan, het ontwijken van obstakels, en interactie in de lucht, beschrijft het laatste deel van dit proefschrift een nieuwe autonome regelaar voor vliegende manipulator. Deze regelaar is gebaseerd op de uniforme dynamiek van een vliegende manipulator. Bovendien is de gebruikte dynamiek van de robot manipulator toegevoegd aan de vliegende robot. Resultaten tonen aan dat deze regelaar de stabiliteit robuustheid van de aangestuurde vliegende manipulator aanzienlijk verbetert en de wendbaarheid vergeleken met de huidige stand van de techniek vergroot. 


\title{
List OF ACRONYMS
}

\author{
AOA Angle of Attack \\ COM Center of Mass \\ DFMAV Ducted-fan Miniaturized Aerial Vehicle \\ DOF Degree of Freedom \\ EMG Electromyography \\ GPS Global Positioning Systems \\ PID Proportional-Integral-Derivative \\ PD Proportional and Derivative \\ ROS Robotic Operating Systems \\ UAV Unmanned Aerial Vehicle \\ UDP User Datagram Protocol \\ VTOL Vertical Take-off and Landing
}





\section{Contents}

Acknowledgment $\quad$ i

Summary $v$

Samenvatting vii

List of Acronyms ix

1 Introduction $\quad 1$

1.1 Aerial Service Robots . . . . . . . . . . . . . . . . . . . . 2

1.2 Control of Aerial Service Robots . . . . . . . . . . . . . . . . 4

1.3 About This Thesis . . . . . . . . . . . . . . . . . 6

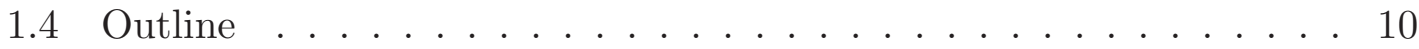

2 A Generic Hapitc Tele-control Architecture for Aerial Robots 13

2.1 Introduction . . . . . . . . . . . . . . . . . . . . . . . 14

2.2 Background . . . . . . . . . . . . . . . . . . . 16

2.3 Teleoperation Control Architecture . . . . . . . . . . . . . . 18

2.4 Virtual Slave System . . . . . . . . . . . . . . . . . . . . . . . . . . . . . . . . . . . . . .

2.5 Real Slave System . . . . . . . . . . . . . . . . . . . . . . . . . . . . . . . . . 25

2.6 Master System . . . . . . . . . . . . . . . . . 26

2.7 Passivity Analysis . . . . . . . . . . . . . . . . . . . . 32

2.8 Simulations . . . . . . . . . . . . . . . . . 34

2.9 Experiments . . . . . . . . . . . . . . . . . . 37

2.10 Conclusion . . . . . . . . . . . . . . . . . . 42

3 Transparency in Haptic Teleoperation of Aerial Robots 45

3.1 Introduction . . . . . . . . . . . . . . . . . 46

3.2 Related Works . . . . . . . . . . . . . . . . . 47

3.3 Transparency in Mobile Robots . . . . . . . . . . . . . . . . 50

3.4 Tele-control Architecture to Enhance Transparency . . . . . . . . . . 54

3.5 Practical Considerations . . . . . . . . . . . . . . . . . 58

3.6 Simulations . . . . . . . . . . . . . . . . . 59 
3.7 Experiments . . . . . . . . . . . . . . . . . . . 64

3.8 Discussion . . . . . . . . . . . . . . . . . . . . . . . . . 73

3.9 Conclusion ....................... 76

4 Multimodal Tele-control architecture for Aerial Robots $\quad 79$

4.1 Introduction . . . . . . . . . . . . . . . . . 80

4.2 Background . . . . . . . . . . . . . . . . . . 83

4.3 The Slave Control Architecture . . . . . . . . . . . . . . . . . 87

4.4 The Master Control Architecture and MultiModal Feedback . . . . . 94

4.5 Practical Considerations . . . . . . . . . . . . . . . . . . 98

4.6 Simulations . . . . . . . . . . . . . . . . . . . 100

4.7 Experiments . . . . . . . . . . . . . . . . . 106

4.8 Conclusion . . . . . . . . . . . . . . . . . . 110

5 A Control Architecture for Aerial Manipulators 113

5.1 Introduction . . . . . . . . . . . . . . . . . . 114

5.2 Dynamics of an Aerial Manipulator . . . . . . . . . . . . 115

5.3 Problem Formulation . . . . . . . . . . . . . . . . . . . . . . 118

5.4 Controller Design . . . . . . . . . . . . . . . . . . . 121

5.5 Stability Analysis . . . . . . . . . . . . . . . . . . 125

5.6 Simulations . . . . . . . . . . . . . . . . . . 127

5.7 Experiments . . . . . . . . . . . . . . . . . . 130

5.8 Discussion . . . . . . . . . . . . . . . . . . . . . . . . . . . . . . 132

5.9 Conclusion . . . . . . . . . . . . . . . . 133

6 Conclusions and Recommendations $\quad \mathbf{1 3 5}$

6.1 Conclusions . . . . . . . . . . . . . . . . . . . 135

6.2 Recommendations ...................... 140

$\begin{array}{lr}\text { Appendices } & 143\end{array}$

A Modeling and Control of underactuated Aerial Robots $\quad \mathbf{1 4 5}$

A.1 Introduction . . . . . . . . . . . . . . . . . . . . 146

A.2 Dynamic Model . . . . . . . . . . . . . . . . . . 146

A.3 Controller Design . . . . . . . . . . . . . . . . . . . . 148

A.4 Results . . . . . . . . . . . . . . . . . . . . 152

A.5 Conclusions . . . . . . . . . . . . . . . . . 155

$\begin{array}{ll}\text { B Parameter Tuning } & 157\end{array}$

$\begin{array}{lrr}\text { C Proof of Proposition } 4.3 & 159\end{array}$

$\begin{array}{ll}\text { About the Author } & 173\end{array}$ 


\section{CHAPTER 1}

\section{INTRODUCTION}

In the early days of robotics, robots, specifically robotic manipulators, have been primarily used in the field of industrial manufacturing for repetitive tasks. Although these robots have significantly improved the quality and quantity of throughput, far beyond that can be achieved by humans alone, their application spectrum was limited as they often need well structured environment and execute their task in a limited workspace [108].

The human desire to explore unstructured and unknown environments without endangering human life, while reducing cost, has led to the development of mobile robots. The term "mobile robot" refers to a large class of unmanned robots that achieve mobility by various methods, such as walking, swimming, and flying. Depending on the environment in which they travel, these robots are broadly classified into ground robots, underwater robots, and aerial robots. Often, however, mobile robot refers to ground robots that achieve locomotion by using wheels or articulated legs [46], [57].

Apart from the mobility freedom they provide in industrial environment, their invention has "opened the doors to the fields", literally. Mobile robots and mobile manipulators (mobile robots on which robotic manipulators are mounted) have been widely used in field applications, such as crop harvesting, land mining, and space exploration [107]. One of the limitations of mobile robots, in the sense of ground robots, is that they are restricted to the surface on which they move. Aerial robots, on the other hand, can virtually navigate through unlimited workspace without being constrained on the ground. The main challenges in dealing with aerial robots are their complex dynamics, limited payload capabilities, and energetic autonomy.

On the bases of how they sustain their flights, aerial robots are often classified as fixed-wing and rotory-wing. Fixed-wing aerial robots include airplanes that use their relative speed and fixed-wing to generate the required lift. They often 


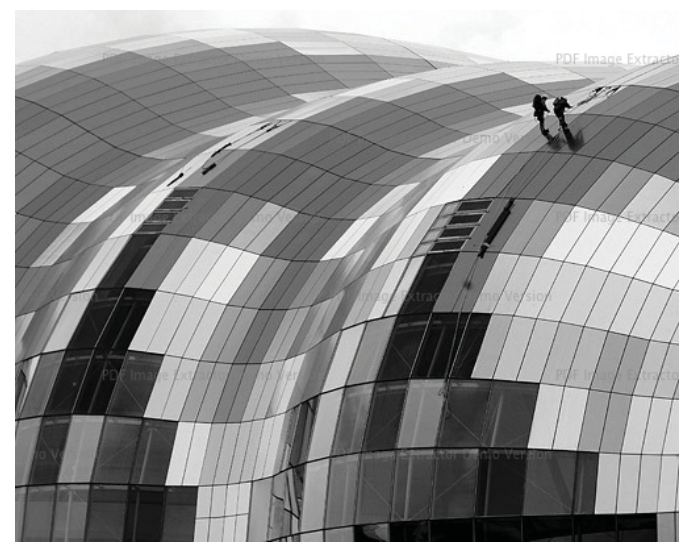

(a) Cleaning (http://www.time.com).

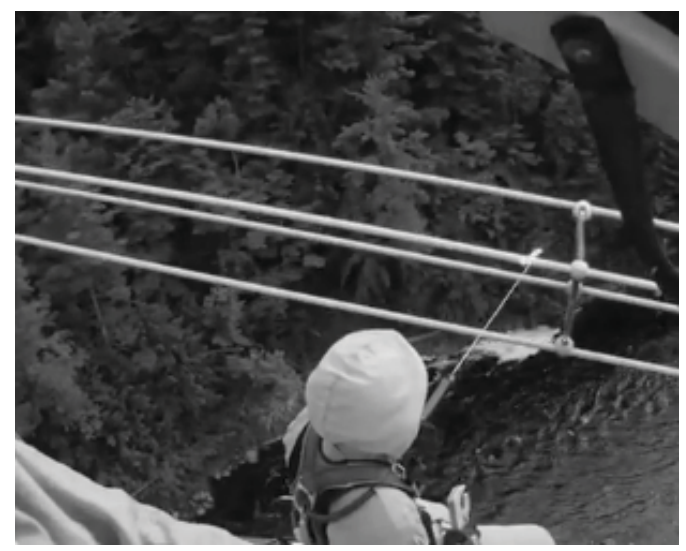

(b) Inspection of high voltage power cables (http://www.haverfield.com).

Figure 1.1: Representative application areas, where aerial service robots can be considered.

require runways to generate the required relative speed for lift-off. On the other hand, rotary-wing aerial robots, in which helicopters are the most well-known example, use the thrust generated by their rotors to propel themselves through the air. Rotary-wing aerial robots are often capable of vertical take-off and landing (VTOL), side-slip, and low-speed flight, hence do not need runways. For this reason, they are widely preferred over their fixed-wing counterparts, particularly for industrial applications in compact environments. Such industrial applications are the main motivations behind the research project that this thesis work has been a part of. Hence, in this thesis, aerial robots primarily refers to unmanned rotarywing aerial vehicles with VTOL capabilities.

\subsection{Aerial Service Robots}

Traditionally, aerial robots have been used in applications that do not require interaction with the environment, such as surveillance, forest fire detection, search and rescue [107]. In essence, avoiding physical interaction is at the core of the control objective. Recently, however, there is a paradigm shift that aims at using aerial robots for applications that involve active but nondestructive interaction with the environment, especially in the field of service robotics.

Service applications where aerial robots can be considered include those tasks which had been exclusively reserved for humans until recently, such as power plant inspection and cleaning skyscraper windows, see Fig. 1.1. The increase in interest to use aerial robots for service applications is fueled partly by technological advancement, but mainly by end-users such as inspection service providers, who foresee their significant benefits. The deployment of aerial robots for inspection, among others, prevents endangering the lives of human personnel, and reduces inspection costs. For instance, power plant inspection should be performed periodically, and it is often done by partially or completely shutting down the plant 


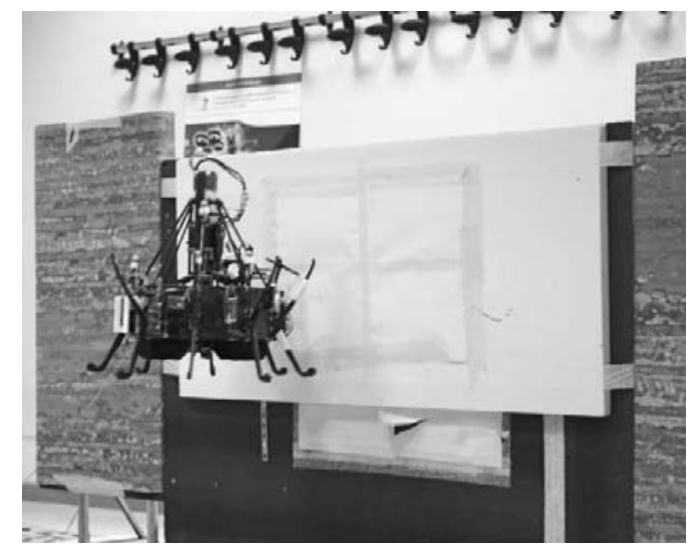

(a) The AIRobots' ducted-fan UAV, endowed with a robotic manipulator, docking on a vertical surface.

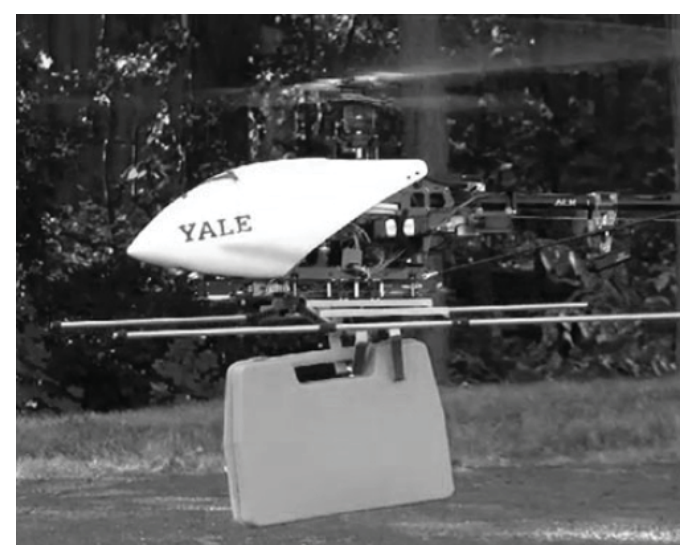

(b) Yale University's minaturized helicopter, endowed with a gripper, transporting a load.

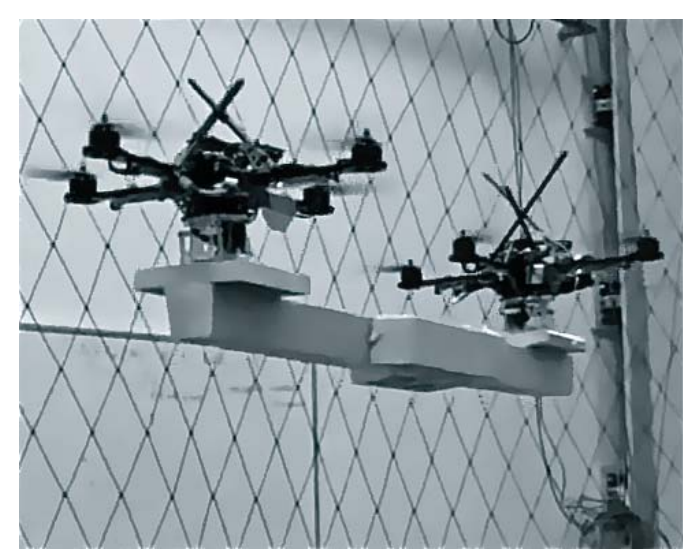

(c) University of Pennsylvania's quadrotors, endowed with grippers, cooperatively transporting a load.

Figure 1.2: Examples of aerial service robots in action.

to avoid endangering the lives of the inspecting personnel. The length of the shutdown is generally proportional to the reduction in the output of the plant, which has direct financial consequences. Furthermore, even in shutdown, a significant number of service personnel get injured or lose their lives while in action. Additionally, not every part of the plant is accessible for inspection, a condition that eventually leads to a reduced life time of the plant. Hence, alternative inspection methodologies that reduce the shutdown time, and do not endanger life are obviously preferred. In this context, aerial robots are poised to be the fundamental parts of tomorrow's inspection technologies.

In general, aerial service robots can be defined as aerial robots aimed at assisting or replacing humans in service applications that require active interaction with the environment, without being constrained on the ground. This newly emerging robot technology has recently attracted roboticists due to its potential applications as well as the related complex scientific challenges. This is manifested by the increase in 
research activities that have been undertaken by growing number of groups around the world [81], [64], [113], [71]. Fig. 1.2 shows some of the resounding recent achievements in the area of aerial service robotics.

These various research groups have been attempting to address different aspects of aerial service robots, such as design of the flying platforms [80], [98], [39], design of manipulation systems [43], [85], sensory fusion [9], [10], control of aerial robots and aerial manipulators [21], [72]. Unified integration and implementation of all these aspects contribute towards partial or complete replacement of the human, and/or effective collaboration of the aerial service robots with the human.

In this thesis, a distinction between aerial robots and aerial manipulator is made. Aerial robot refers to aerial platforms with different mechanical configuration with VTOL capabilities, whereas aerial manipulator refers to any aerial robot on which a robotic manipulator/s of any sort is mounted.

\subsection{Control of Aerial Service Robots}

The deployment of aerial service robots for real-time applications requires possession of several capabilities, such as flight maintenance, safety, and robustness. In general, service robots should be able to accomplish their tasks when deployed in various environments, including unknown, unstructured and GPS denied environments. Application wise, they should be versatile enough to conduct their tasks in free-flight as well as in interaction, as deemed necessary by the task. Depending on the complexity of the task, both in free-flight and interaction, service robots should work independently or in collaboration with humans.

One of the fundamental ingredients to equip aerial service robots with the aforementioned flexible capabilities is the design of advanced control architecture, which is the focus of this thesis.

\subsubsection{Autonomous Aerial Robots}

Aerial robots that are equipped with autonomous control capability are able to accomplish their tasks autonomously, even in the presence of uncertainties that the controller is capable of overcoming. Design of classical autonomous controller for aerial robots is well researched area. Both linear and nonlinear controllers for various mechanical configuration of aerial robots have been designed, [16], [92], [60], [37], [66]. The goal of the controllers include navigation, trajectory tracking and obstacle avoidance.

Design of autonomous aerial interaction controllers, on the contrary, is an emerging research topic and is not yet fully developed. Some of the interesting challenges in control of aerial interaction include dealing with change in dynamics during interaction, stability during a constrained motion, and time-varying interaction force regulation. So far, only few results have been reported, [3], [7], [81]. The state of the art on autonomous aerial interaction control of aerial robots are hybrid position/force controller or impedance controller. The hybrid controllers rely 
on either force measurement data or model-based force estimation for interaction set-point regulation. The main limitation of hybrid controllers in general is their heavy reliance on precise a priori knowledge about their environment or stability issues in case of lack of this knowledge. Impedance controllers, on the other hand, do not suffer from the lack of knowledge about the environment. However, they alone do not intrinsically posses interaction force regulation capabilities. The slave control architecture presented in Chapter 4 of this thesis, addresses the limitations of those controllers by designing a unified variable impedance and force controller for aerial interaction.

Unlike that of aerial robots, design of autonomous controller for aerial manipulators is at its infancy. Limited results have been reported so far [21], [26]. These controllers are mainly aimed at active but safe interaction with the environment. Often, the controllers of the aerial robot and the robotic manipulator are designed independently, disregarding their effect on each other, [91], [102]. Although such an approach is modular in nature, it sometimes significantly limits their performance. To address this limitation, Chapter 5 proposes a novel unified control architecture for aerial manipulators.

\subsubsection{Bilateral Teleoperation}

As the complexity of the tasks that the aerial robots are used for increases, completion of the tasks autonomously becomes difficult, if not impossible. In such situations, incorporating the human in the control loop becomes necessary. Don Norman once said "Roboticists automate what is easy and leave the rest to the human" (www.jnd.org). The inclusion of the human in the control loop brings the astounding human capability of decision-making, perception and adaptability. In general, for the successful completion of the task, effective interaction between the aerial robot and the human is fundamental. To achieve effective interaction, the development of appropriate human-machine interaction interfaces and the design of advanced control algorithms are mandatory. Both are important aspects of the field of teleoperation of robots.

Teleoperation refers to operation of a robot over a distance, in which the distance emphasizes the physical separation between the human and the robot that directly interacts with the environment. Traditionally, vision feedback obtained from an on-board and/or off-board cameras of the aerial robot closes the control loop during teleoperation of aerial robots [20]. However, the emerging aerial service applications are complex and even include active interaction. As a result, situational awareness derived from vision feedback alone may not be adequate enough for completion of a task. Force feedback, on the other hand, in augmentation with vision feedback or alone, restores the sense of direct interaction. In essence, the force feedback results in kinestetically coupling the operator with the environment through master and slave systems.

Teleoperation architectures that consist of force feedback are referred as bilateral teleoperation. Although bilateral teleoperation may in general signify a bi-directional signal flow, it often refers to haptic teleoperation. In this thesis, hap- 
tic and bilateral, teleoperation and tele-control, as well as human operator, user and operator are terms that are used interchangeably in the context of bilateral teleoperation control architectures.

Although it has been more than half a century since the introduction of haptic teleoperation, it is still an active research topic, especially in emerging fields such as aerial robotics. The two main objectives of any tele-control algorithm have been stability and transparency. Loosely speaking, stability measures the degree of robustness in any operating conditions, whereas transparency refers to the degree of performance in timely perception of a desired behavior. Numerous methodologies have been proposed to achieve the two main objectives while addressing classical challenges, such as time delays and packet losses. Nevertheless, the peculiar challenges, due to the type of robots themselves and the evolving complexity of tasks that the robots are aspired to be used for, hinder the direct employment of already developed methodologies. For instance, workspace incompatibility and underactuation of aerial service robot are few of the peculiar challenges. Chapter 2 identifies additional peculiar challenges in this field, and provides practical ways of addressing those challenges as well as the existing classical challenges. The major part of this thesis proposes different advanced tele-control architectures for aerial service robots. Using different mathematical tools, the stability of the proposed architectures and the degree of achievable transparencies are detailed out. Various practical issues that may arise during implementation phase and methods to tackle these issues are also discussed.

\subsection{About This Thesis}

\subsubsection{Objectives}

The main objective of this thesis is the development of new and advanced control architectures for human-machine interaction that enhance the performance of the human operator while performing complex service tasks using aerial robots. While the main focus of the thesis is on bilateral teleoperation, it also includes the design of a novel autonomous controller for aerial manipulators.

The specific goals of this thesis include

- Identifying peculiar challenges in haptic teleoperation of aerial robots, and proposing methods to address both classical and peculiar challenges.

- Designing haptic teleoperation control architectures that are stable and highly performing in free-flight and interaction tasks.

- Designing generic control architectures that are not dependent on the type of aerial robot or haptic interface.

- Designing advanced autonomous control architecture for aerial manipulators that efficiently exploit the robotic manipulators appended to them, which are primarily intended for interaction tasks. 


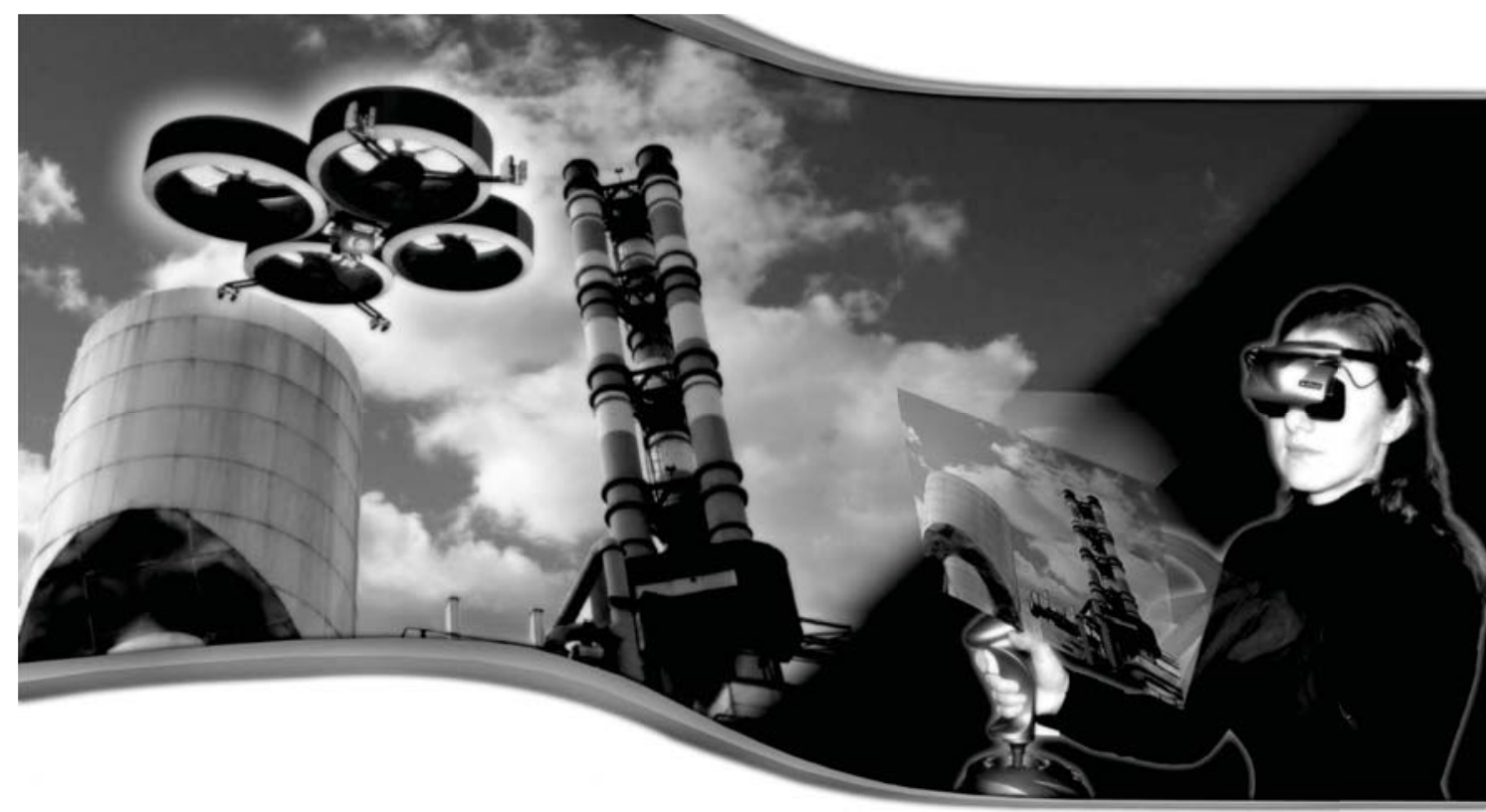

Figure 1.3: Illustration of the AIRobots project vision.

- Validating and verifying the feasibility and efficiency of the proposed control architectures in simulations and experiments in realistic scenarios that can be cornerstones for real-time applications.

\subsubsection{The AIRobots project}

The research presented in this thesis has been conducted as part of the European project entitled "Innovative Aerial Service Robots for Remote Inspection by Contact (AIRobots)". The goal of the AIRobots project is to develop a new generation of aerial service robots that are capable of supporting human beings in all activities which require the ability to interact actively and safely with the environment without being constrained on ground (http://airobots.ing.unibo.it). The step forward with respect to the "classical" field of aerial robotics is to realize aerial vehicles and a control framework that are able to accomplish a large variety of service applications, such as inspection of buildings and large infrastructures, sample picking, and aerial manipulation, autonomously and/or with the human in the control loop, [59].

A graphical sketch of the vision of AIRobots is shown in Fig. 1.3. The aerial robot, equipped with appropriate sensing devices including robotic manipulators, is remotely controlled by means of haptic devices, which allow the operator to remotely supervise the task. The operator is assumed to be a specialist in the service task rather than a pilot.

In this scenario, integrated design schemes between the remote operator and on-board automatic control have been studied according to schemes that are not fixed a priori, but modified according to evolving needs and conditions. As part 


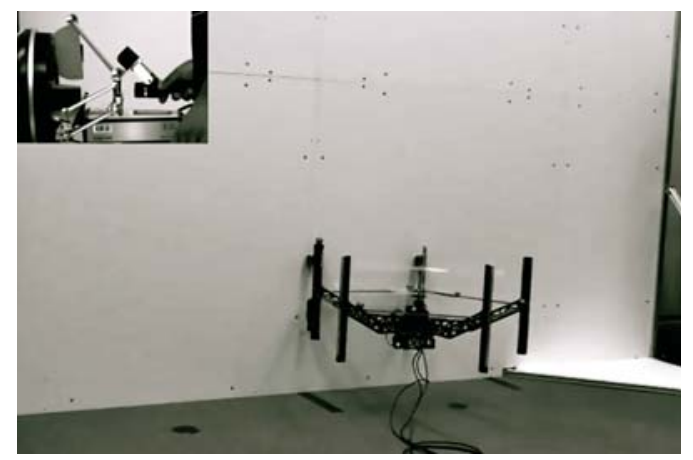

(a) Teleoperation of the AIRobots' coaxial rotor-craft for interaction tasks.

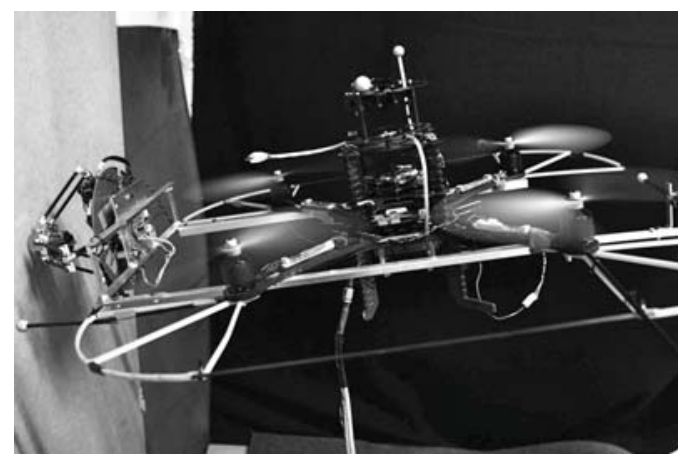

(b) The Asctec pelican's quadrotor, endowed with a robotic manipulator, in interaction task.

Figure 1.4: Aerial platforms used in the AIRobots project.

of the verification process of the applicability of the proposed control algorithms, both autonomous and teleoperation, are tested on minaturized VTOL aerial robots either developed as part of the AIRobots project, such as ducted-fan and coaxial rotorcraft, or commercially available, such as quadrotors, see Figs. 1.2a and 1.4. All the results obtained from the project are intended to provide deeper insight in the complexity and real-time challenges of working with aerial service robots in industrial scenarios. The results are used as fundamental bases for the necessary engineering steps that are required to use the robots for real-time civilian applications.

\subsubsection{Contributions of This Thesis}

With respect to the stated goals in Section 1.3.1, in the context of the AIRobots project and relative to the current state of the art, the main contributions of this thesis are presented in this section.

This thesis provides theory and application of advanced human-robot interaction control architectures that enhance the performance of the human operator while performing complex service tasks using aerial robots. The architectures are designed to perform efficiently in challenging operating conditions that involve an aerial service robot, which acts as flying extensions or replacement of the operator's hand, possibly deployed in a remote and unstructured environment and may involve in active interaction with the environment. Generic bilateral teleoperation algorithms that attempt to create improved telepresence of the human operator, ensure operator and robot safety in the presence of various classical and peculiar challenges, and enable the operator to accomplish a task efficiently with less physical and mental load are presented and discussed in detail. Moreover, an efficient autonomous controller for aerial manipulators is also presented. The development of all the proposed control algorithms include detailed theoretical analysis and extensive simulations and experimental results that illustrate the feasibility and performance of the control architectures in various realistic operating conditions. 
While the algorithms proposed are primarily geared towards the field of aerial service robots, they are generic in nature and are applicable to other fields of robotics. Moreover, the slave controllers designed in the context of bilateral teleoperation can also be used for autonomous control with or without slight adaptation.

The main specific contributions of this thesis are summarized as follows.

- A generic control framework for haptic teleoperation of aerial service robots that achieves improved transparency and remains passive even in the presence of significant network-induced imperfections.

- A versatile multimodal variable impedance control architecture for haptic teleoperation of aerial robots that is capable of regulating time-varying interaction force.

- A novel control architecture for aerial manipulators that exploits the dynamics of a robotic manipulator to improve the maneuverability of the aerial robot.

\subsubsection{List of Publications}

Most of the results of the research described in this thesis are presented in the following articles.

\subsubsection{Journal Articles}

- Mersha, A. Y., Fumagalli, M., Stramigioli, S., and Carloni, R., "A new control architecture for aerial robots: adapt and exploit", IEEE Transactions of Robotics, 2014, In preparation.

- Mersha, A. Y., Xiaolei, H., Mahony, R., Stramigioli, S., and Carloni, R., "Transparency in haptic teleoperation of aerial robots", IEEE Transactions of Robotics, 2014, Under review.

- Mersha, A. Y., Fumagalli, M., Stramigioli, S., and Carloni, R., "Multimodal variable impedance control for haptic teleoperation of interactive aerial robot", The International Journal of Robotics Research, 2014, Under review.

- Mersha, A. Y., Stramigioli, S., and Carloni, R., "On bilateral teleoperation of aerial robots", IEEE Transactions of Robotics, Vol. 30, no. 1., pp. 258274,2014 .

- Carloni, R., Lippielo,V., Massimo D’Auria, Fumagalli, M., Mersha, A. Y., Stramigioli, S., and Siciliano, B., "Robot vision: obstacle-avoidance techniques for unmanned aerial vehicles", IEEE Robotics and Automation Magazine, Vol. 20, no. 4, pp. 22-31, 2013. 


\subsubsection{Conference Papers}

- Mersha, A. Y., Stramigioli, S., and Carloni, R., "A variable impedance control for aerial interaction", In Proceedings of the IEEE International Conference on Intelligent Robots and System, 2014.

- Mersha, A. Y., Stramigioli, S., and Carloni, R., "Exploiting the dynamics of a robotic manipulator for control of UAV", In Proceedings of the IEEE International Conference on Robotics and Automation, 2014.

- Mersha, A. Y., Xiaolei, H., Mahony, R., Stramigioli, S., Corke, P., and Carloni, R., "Intercontinental haptic teleoperation of a flying vehicle: a step towards real-time applications", In Proceedings of the IEEE International Conference on Intelligent Robots and Systems, 2013.

- Mersha, A. Y., Rüesch, A., Stramigioli, S., and Carloni, R., "A contribution to haptic teleoperation of aerial vehicles", In Proceedings of the IEEE International Conference on Intelligent Robots and Systems, 2012.

- Mersha, A. Y., Stramigioli, S., and Carloni, R., "Switching-based mapping and control for haptic teleoperation of aerial robots", In Proceedings of the IEEE International Conference on Intelligent Robots and Systems, 2012.

- Mersha, A. Y., Stramigioli, S., and Carloni, R., "Bilateral teleoperation of underactuated aerial vehicles: the virtual slave concept", In Proceedings of the IEEE International Conference on Robotics and Automation, 2012.

- Rüesch, A., Mersha, A. Y., Stramigioli, S., and Carloni, R., "Kinetic scrolling-based position mapping for haptic teleoperation of unmanned aerial vehicles", In Proceedings of the IEEE International Conference on Robotics and Automation, 2012.

- Mersha, A. Y., Carloni, R., and Stramigioli, S., "Modeling and control of underactuated aerial vehicles", In Proceedings of the IEEE International Conference on Robotics and Automation, 2011.

\subsection{Outline}

The main chapters of this thesis are composed of slightly adapted and self-contained versions of the articles listed in Section 1.3.4. Though there is a repetition of some information for self-containment of each chapter, the chapters are organized in a coherent manner to provide a unified theoretical development and practical contributions, with respect to the goals stated in Section 1.3.1. The outline of this thesis is as follows.

Chapter 2 presents a generic passive control architecture for haptic teleoperation of aerial robots. It is characterized by distinct high-level teleoperation and low-level regulation loops. The architecture addresses both classical and peculiar challenges 
of haptic teleoperation of aerial robots, and its features make it applicable in wide range of applications. It works in a plug-and-play fashion with any type of haptic device, aerial robot and its low-level controller without compromising its passivity.

Chapter 3 defines transparency for teleoperation of mobile robots, both in freemotion and interaction. It provides mathematical definition of vehicle and environment transparencies, and their measure of deviation from ideal transparency. It also investigates methodologies of achieving improved performance in the presence of significant network-induced imperfections. The control architecture is realized in the longest intercontinental setting involving master and slave systems located in the University of Twente, the Netherlands, and the Australian National University, Australia.

Chapter 4 primarily focuses on teleopration of aerial robots involved in interactive tasks. It proposes a multimodal variable impedance interaction controller, which is also capable of regulating time-varying interaction force. Both the impedance and the interaction force are modulated by the human operator. Moreover, a multimodal sensory feedback, which includes force and tactile, are fed back to the human operator to increase awareness of the remote environment, thereby, increasing the task-performance.

Chapter 5 slightly deviates from the other chapters and presents an autonomous free-flight control architecture for aerial manipulators. The framework exploits the dynamics of task-specific robotic manipulators appended to them for secondary tasks. It is primarily used to improve the tracking performance and maneuverability of the aerial robot by widening its flight envelop. The benefit of the proposed framework relative to the state of the art with respect to stability boundaries, tracking performance and parametric properties of the manipulator are also discussed. The analysis provides more insight on how to efficiently exploit the dynamics of the manipulator during control design and mechanical design phases.

The last chapter summarizes the main contributions of this thesis by putting into perspective the conclusions drawn in each chapter. It also provides outlooks on extensions and different directions for possible future research. 



\section{CHAPTER 2}

\section{A Generic Hapitc Tele-control Architecture FOR AERIAL RoBots}

This chapter presents a generic hierarchical passive teleoperation control architecture that effectively addresses the issues of workspace incompatibility and precision, as well as other classical and peculiar challenges. More specifically, the control scheme consists of a user-defined variable scale mapping, a variable impedance master controller and a virtual slave system. The port-based modeling framework has been extensively used in the formulation, providing more insight about energetic flows in the system that are particularly useful for the design of a passive controlled system. Moreover, various practical considerations that are required for the effective usage of the control architecture are discussed. The achieved better precision and overall task performance have been validated and verified by elaborate simulations and experiments.

This chapter is a slightly adapted version of (Mersha et al. 2014a), A.Y. Mersha, S. Stramigioli and R. Carloni, "On Bilateral Teleoperation of Aerial Robots", IEEE Transaction on Robotics, Vol. 30, no. 1., pp. 258-274, 2014. 


\subsection{Introduction}

In the past decade, in the robotic community, there has been great interest in the field of Unmanned Aerial Vehicles (UAVs), particularly in the class of Vertical Take-off and Landing (VTOL) UAVs such as quadrotors, coaxial-rotors and ducted-fans [91], [16], [80]. The ubiquity of VTOL UAVs is mainly attributed to their superior agility, hovering capability and smaller size. Moreover, the surge in interest, along with recent technological advancements in miniaturization of computational boards, sensors and power sources, has been very instrumental for the recent advances in the field.

So far, most of the research works have mainly focused on mechanical design [91], [80] and autonomous controller design [16], [37], [66]. The newly emerging research line in the field, however, has shifted the focus of application from only using UAVs as pilotless aerial vehicles to using them as aerial platforms to perform robotic activities. This is attributed to the rapid expansion of potential application areas in the civil sector such as, power plant inspection by contact, object manipulation, and disaster management.

Deploying UAVs in complex application areas with complete autonomy is not possible or even desirable for various reasons. As a consequence, teleoperated flights, with "human-in-the-loop" control architectures, are beneficial in accomplishing complex tasks. One of the main challenges in teleoperation of UAVs is the reduced situational awareness of the operator due to the lack of multiple sensory feedbacks that could be available for an on-board pilot [47]. To overcome this challenge, the first research in the field used vision feedback by either keeping the UAV in line of sight of the operator or using images coming from on-board cameras [20], [96]. However, the limited resolution and field of view of on-board cameras, high dependency on weather condition, and high transmission latency of image data have resulted in a reduced situational awareness. Recently, some research works have shown that the use of force feedback without or in augmentation with vision feedback in teleoperation of UAVs increases safety and performance [47], [69], [94].

\subsubsection{Peculiar Challenges}

Well-known challenges in classical haptic teleoperation of robotic systems include network-induced imperfections such as time delays and packet losses, which are primary sources of instability and lack of transparency. Many researchers have tried to address these issues since the introduction of teleoperation systems half a century ago [82], [23].

Compared to classical bilateral teleoperation, haptic teleoperation of UAVs poses additional and interesting challenges. The main peculiar challenges, along with methods proposed to address them in the literature, are briefly reviewed as follows.

Master-Slave Kinematic Dissimilarity: Unlike in most classical bilateral teleoperation systems, the master is neither an exact nor a scaled replica of the slave. Furthermore, there exists incompatibility between the bounded and unbounded 
workspaces of the master and slave devices, respectively. Consequently, only direct/scaled state mapping is not applicable for most tasks.

To overcome the master workspace limitation, a car driving metaphor (rate control), i.e., a mapping of the position of the master to the velocity reference of the slave, has been adopted by several authors [47], [69], [94], [111], [22]. Such a mapping strategy is also extensively employed in the field of ground mobile robots [14], [54]. In a rate control strategy, even in the absence of the operator's input, any deviation in the master device from its neutral position may result in an uncontrolled movement of the UAV. In [94], the master controller is designed to explicitly take this condition into account with a control loop closed on the master side. However, the additional control loop creates an additional task for the master controller, whose main objective is displaying haptic feedback based on the state of the UAV or its surrounding environment. This additional controller action may generate a high force, which, as a consequence, limits the force displaying capability and compromises the fidelity of the haptic feedback.

Moreover, rate control compromises the achievable precision, particularly in the presence of network-induced imperfections. As a result, employing this strategy is difficult, if not impossible, in application areas where precision is of an essence, such as object manipulation, sample picking, scratching, and cleaning. To address the workspace and precision issues, a kinetic scrolling based position mapping algorithm has been proposed in [97]. The algorithm is inspired by the kinetic scrolling of smart phones. The main drawback in this algorithm is that the operator has to constantly switch between the modes to cover fairly large workspaces and to change the direction of the trajectory [68].

Underactuatedness of UAVs: Most of the currently existing flying platforms are underactuated (less number of actuators than the DOFs). Generally, one is often interested in teleoperating the translational DOFs, of which, $x$ and $y$ are not directly actuated. As a consequence, it is not possible to directly apply the exact required forces onto these DOFs. Moreover, letting the operator deal with the underactuation directly results in high cognitive overload, as the operator has to solve the underactuation problem.

Virtual Force: In maneuvering tasks, there is no active and direct interaction between the UAV and the environment. So, the haptic feedback does not come from physical contact with the environment, but rather from an artificial potential that provides intuitive cues about the state of the UAV and its surrounding environment, e.g., obstacles.

As yet, the haptic feedback in teleoperation of UAVs comes from an artificial potential field built around obstacles [47], [94], [48], [49] or deviation of the state of the UAV from the desired ones [111], [97] or both [69], [70]. Often, the artificial potential field is built based on sensory feedback, and the generated force feedback indicates how close the UAV is to the obstacle, i.e., the closer the UAV to the obstacle, the higher the force becomes.

Measurement Signals Limitation: It is usually difficult to measure the aerodynamic variables of UAVs with acceptable accuracy. Most measurement feedbacks are limited to estimates of the rigid body states of the UAV [111]. 
Continuous Energy Dissipation: Most UAVs continually dissipate energy even while nearly hovering due to gravity. Hence, direct energetic coupling, as is done in classical bilateral teleoperation, between the master and the real slave system requires infinite energy supply from the operator.

Operator Perception and Control: Human operators have limited capability in simultaneously perceiving and controlling many DOFs of a slave device. They perform well when the number of DOFs that they directly control is few.

\subsubsection{Contribution}

This chapter presents a theoretical formulation and practical realization of a generic passive controller for haptic teleoperation of aerial robots that comprehensively addresses both peculiar and classical challenges in the field. The controller is characterized by its clear distinction between the high-level bilateral teleoperation and the low-level dynamic regulation control loops. The presence of the userdefined variable scale mapping strategy, the variable impedance master controller and the virtual slave system in the teleoperation loop makes it applicable to a wide range of tasks and operating conditions. Furthermore, the multidimensional treatment of the control scheme adds to its generic nature.

Moreover, simulations and experimental results are provided, validating the applicability and effectiveness of the algorithm. Apart from that, detailed practical considerations and implementation issues are discussed. System integration of various technological components that are required to practically realize the proposed control scheme is also presented.

The rest of the chapter is organized as follows. In Section 2.2, a brief background on the port-based modeling framework is presented. In Section 2.3, the teleoperation control architecture is introduced. The main parts of the proposed teleoperation scheme, which include the variable impedance master controller and the virtual slave, are detailed in Sections 2.4-2.7. Validating simulations and experimental results illustrating the performance of the proposed control structure in various operating conditions are given in Sections 2.8 and 2.9. Finally, concluding remarks are provided in Section 2.10.

\subsection{Background}

In this section, a brief background on the port-based modeling framework, which is extensively used in our formulation, is provided. In particular, port-Hamiltonian systems and bond graphs are briefly summarized.

In the port-based framework, a dynamic physical system is modeled as an interconnection among few basic elements that exhibit specific energetic behavior and interact through power ports in an energetically consistent way. The power ports are described by power conjugate variables, called effort $e$ and flow $f$, whose dual product represents power. For instance, in the mechanical domain, the wrench $W$ acting on a body and the twist $T$ of a body are the effort and flow port variables, whose dual product gives the mechanical power transferred to the body. 


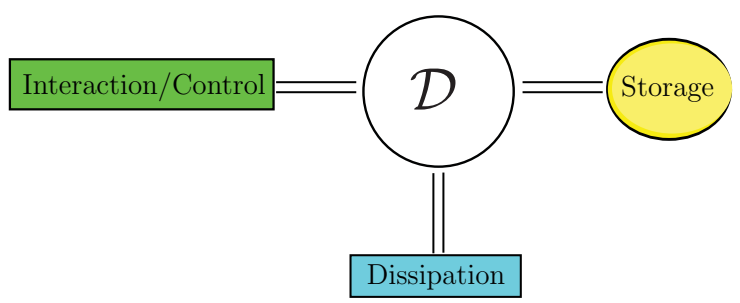

Figure 2.1: Network structure of a generic physical system. The Dirac structure $\mathcal{D}$ defines the power continuous interconnection between the different elements. The internal energy storage element can be either a generalized momentum or a configuration storage element, and the energy dissipative element can be of any element with dissipative action. Interaction/control port represents the port through which the system interacts with the environment and by which the control action is carried out.

\subsubsection{Port-Hamiltonian Systems}

The main ingredients of a port-Hamiltonian system are the state manifold $\mathcal{X}$ of the energy variables $x$ and the Dirac structure $\mathcal{D}$ [15]. A smooth energy function $\mathcal{H}(x): \mathcal{X} \rightarrow \mathbb{R}$, called the Hamiltonian of the system, is assigned to each energy state $x \in \mathcal{X}$ of the system. The rate at which this energy changes, i.e., $\dot{\mathcal{H}}$, is obtained by the dual product of the co-vector $d \mathcal{H}=\frac{\partial H(x)}{\partial x}$ and the vector $\dot{x}$. A port-Hamiltonian system interacts with the external world through its power ports. The power distribution network among the basic energetic elements and the external world is defined by a Dirac structure, which is a mathematical object that describes a power conserving network topology of the system.

Port-Hamiltonian systems are acausal, where the cause and the effect are not known a priori. However, for computational reason, they are usually made causal. The dynamic equation of a generic causal port-Hamiltonian system is described as

$$
\left(\begin{array}{c}
\dot{x} \\
-y
\end{array}\right)=\left(\left[\begin{array}{cc}
J_{1}(x) & g(x) \\
-g^{T}(x) & J_{2}^{T}(x)
\end{array}\right]-\left[\begin{array}{cc}
R_{1}(x) & 0 \\
0 & -R_{2}(x)
\end{array}\right]\right)\left(\begin{array}{c}
\frac{\partial H(x)}{\partial x} \\
u
\end{array}\right)
$$

where $J_{1}, J_{2}$ are skew-symmetric matrices; $g(x)$ is an input matrix $g(x) ; R_{1}(x)$ and $R_{2}(x)$ are positive definite matrix denoting the dissipation; $u$ is the input and $y$ is the passive output of the system. Fig. 2.1 shows a graphical representation of a generic port-Hamiltonian systems.

\subsubsection{Bond Graphs}

A bond graph is a domain independent graphical modeling language for dynamic physical systems. It is composed of elements that represent elementary concepts of energy storage, dissipation and transformation. These elements are interconnected by bonds, represented by half arrows that signify the power flow among the elements. Positive power flows in the direction of the arrow.

The similarity in the fundamental foundation of port-Hamiltonian systems and bond graphs, both based on the concepts of energy and power flows among elements, makes bond graphs highly suitable for compact graphical representation of 


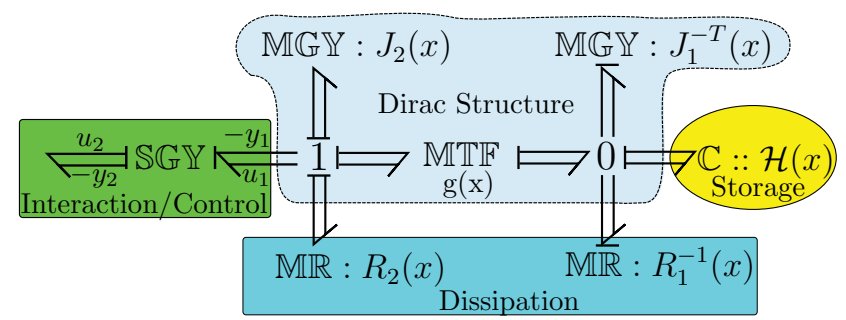

Figure 2.2: Bond graph representation of a port-Hamiltonian system. The Dirac structure is represented by the (Modulated) energy transforming elements denoted by $\mathbb{M T F}$ and $\mathbb{M} \mathbb{G} \mathbb{Y}$, and the junction structures denoted by 0 and 1 . The $\mathbb{C}$ and $\mathbb{R}$ elements represent energy storage and dissipation, respectively. Interaction with the environment and control actions are all collected by the power port denoted by $u$ and $y$. If $u$ and $y$ in (2.1) are effort input and flow output

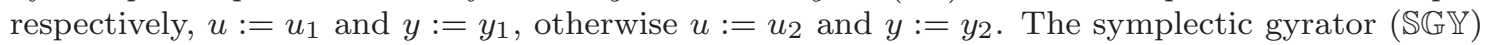
is a gyrator with a transformation ratio of unity and is included only to make the representations more general. Note that the above bond graph representation of port-Hamiltonian systems is not unique.

port-Hamiltonian systems [15]. Representing port-Hamiltonian systems in bond graphs provides a graphical view of the network topology and renders more insight in the system dynamics. This highly facilitates design of control laws by providing more intuition. Fig. 2.2 shows a causal bond graph representation of the portHamiltonian systems, as given in (2.1). It shows the power conserving nature of the Dirac structure and interaction among various dynamical elements.

\subsubsection{Notation}

$\psi^{i} \quad$ A right handed orthonormal coordinate frame $i$.

$H_{j}^{i} \quad$ A $4 \times 4$ homogeneous matrix that transforms coordinate from $\psi^{j}$ to $\psi^{i}$. Mathematically, $H_{j}^{i}=\left(\begin{array}{cc}R_{j}^{i} & p_{j}^{i} \\ 0 & 1\end{array}\right)$, where $R_{j}^{i}$ is a rotation matrix and $p_{j}^{i}$ is a displacement vector.

$T_{j}^{k, i} \quad$ Twist of $\psi^{j}$ with respect to $\psi^{i}$ expressed in $\psi^{k}$.

$W_{i}^{k} \quad$ Wrench applied to a body where $\psi^{i}$ is attached to and expressed in $\psi^{k}$.

$P_{i}^{k} \quad$ Generalized screw momenta of a body where $\psi^{i}$ is attached to and expressed in $\psi^{k}$. $P_{i}^{k}=I_{i}^{k} T_{i}^{k, 0}$, where $I_{i}^{k}$ is a generalized inertial tensor of a body, on which $\psi^{i}$ is attached to and expressed in $\psi^{k}$.

$H(x) \quad$ A Hamiltonian of a system with energy state $x$.

$u$ and $y \quad$ Power port variables representing the input and the corresponding collocated passive output of a system.

Unless otherwise stated or is clear from the expression, vectors and matrices used throughout this chapter are of dimension 4 and $4 \times 4$, respectively.

\subsection{Teleoperation Control Architecture}

Teleoperation systems are primarily designed to transfer the human operator's control/manipulation capability to a possibly remotely located slave system, which 


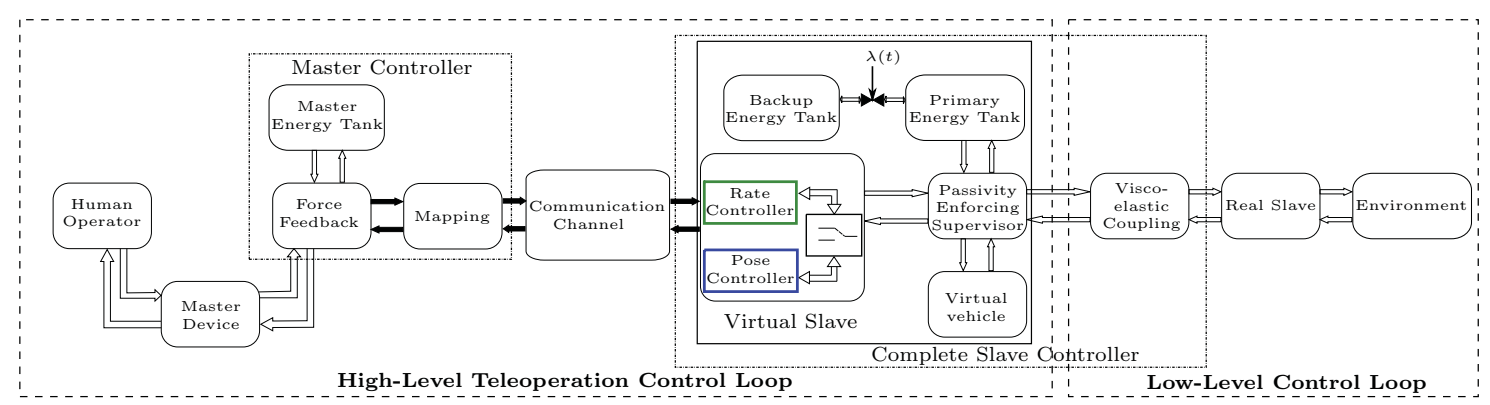

Figure 2.3: Block diagram representation of the overall haptic teleoperation control structure.

directly interacts with the environment, with the use of a master system by maximizing the telepresence of the operator. Both the master and the slave systems are endowed with local controllers. The slave controller generates appropriate control inputs for the slave to follow the operator's command. The master controller, on the other hand, generates force feedback that renders the state of the slave and/or the characteristics of the environment the slave interacts with. The two controllers exchange information through a communication channel, which introduces network-induced imperfections, such as time delays and packet losses.

From the control point of view, the two main control objectives when designing tele-controllers are stability and transparency. In this study, passivity is set as a control objective as it is a sufficient condition for stability and is an intrinsic property of port-Hamiltonian systems.

Fig. 2.3 shows the structure of the proposed control scheme, which is composed of the high-level and low-level control loops. Each subsystem of the complete control scheme is presented in the subsequent sections.

\subsection{Virtual Slave System}

As its name indicates, the virtual slave system is an ideal slave system and it is implemented as part of the real slave's controller (see Fig. 2.3). It serves as a proxy for the real slave and provides numerous merits during haptic teleoperation. As discussed in the subsequent sections, the inclusion of the virtual slave system without much additional computational cost enables to

- hide the underactuation of the real slave;

- energetically couple the master with the slave system;

- facilitate the usability of the proposed teleoperation architecture independently of the flying platform and its associated low-level controller, without compromising the stability of the high-level control loop.

To serve its purpose, the virtual slave dynamically interacts with the master and the slave in a stable way under all operating conditions. It is composed of a multidimensional virtual vehicle, its controller, energy tanks and a passivityenforcing supervisor. 


\subsubsection{Virtual Vehicle}

Intuitively, the virtual vehicle serves as a proxy for the real slave, i.e., the real UAV. For the virtual vehicle to serve as a reliable intermediary between the operator and the real slave, it should reflect the state of the real slave while simultaneously hiding various complexity of the real slave from the operator.

To that end, the virtual vehicle is modeled as a momentum storage element that flies in an ideal environment, where there is neither gravity nor friction [111]. Since it is implemented as part of the real slave's controller, all its states are readily available for use. In addition, it has reduced DOFs, i.e., only the DOFs that the operator can and want to have control over, often all the translations $(x, y$ and $z)$ and the yaw $\left(\theta_{z}\right)$. In other words, the virtual vehicle has less DOFs than the real slave, and it is fully actuated unlike the underactuated real slave. As such, the underactuation of the real slave is hidden from the operator, which could result in high cognitive overload otherwise. Moreover, having reduced DOFs decreases the additional computational cost incurred due to the inclusion of the virtual slave. In this study, the virtual vehicle has four DOFs, in accordance with the characteristic feature of most of the currently existing underactuated flying vehicles.

Let the inertial coordinate frame be $\psi^{0}$, and the body fixed frame $\psi^{b v}$, which is attached to the COM of the virtual vehicle (see Fig. 2.4). The dynamics of the virtual vehicle is described by

$$
\left\{\begin{aligned}
\dot{\bar{P}}_{b v}^{b v} & =\widetilde{\bar{P}}_{b v}^{b v} \frac{\partial H\left(\bar{P}_{b v}^{b v}\right)}{\partial \bar{P}_{b v}^{b v}}+\bar{W}_{b v}^{b v} \\
\bar{T}_{b v}^{b v, 0} & =\frac{\partial H\left(\bar{P}_{b v}^{b v}\right)}{\partial \bar{P}_{b v}^{b v}}
\end{aligned}\right.
$$

where $H\left(\bar{P}_{b v}^{b v}\right)=\frac{1}{2}\left(\bar{P}_{b v}^{b v}\right)^{T}\left(\bar{I}_{b v}^{b v}\right)^{-1} \bar{P}_{b v}^{b v} ; \bar{W}_{b v}^{b v}=\left[\tau_{b v-z}^{b v}, F_{b v-x}^{b v}, F_{b v-y}^{b v}, F_{b v-z}^{b v}\right]^{T}$ and $\bar{T}_{b v}^{b v, 0}=\left[\omega_{b v-z}^{b v}, v_{b v-x}^{b v}, v_{b v-y}^{b v}, v_{b v-z}^{b v}\right]^{T}$ denote the 4 -D port variables, i.e., the resultant wrench acting on the virtual vehicle and the corresponding twist.

$$
\widetilde{\bar{P}}_{b v}^{b v}=\left[\begin{array}{cccc}
0 & -P_{b v}^{b v}[3,1] & P_{b v}^{b v}[2,1] & 0 \\
P_{b v}^{b v}[3,1] & 0 & 0 & 0 \\
-P_{b v}^{b v}[2,1] & 0 & 0 & 0 \\
0 & 0 & 0 & 0
\end{array}\right]
$$

is a skew symmetric matrix representing the power conserving network interconnection of the system. The dynamics of the virtual vehicle is equivalent to the dynamics of a rigid body, whose rotational dynamics around the $x$ - and $y$-axes are constrained.

To reliably convey the state of the real slave and the command of the operator, the virtual vehicle is energetically coupled with both of them through a viscoelastic coupling and a local controller, respectively. $\bar{W}_{b v}^{b v}$ in (2.2) is the resultant wrench due to these couplings. In this way, the virtual vehicle dynamically maps the operator's command to the slave, and the real slave's reaction back to the operator. To increase the reliability performance of the virtual slave, its parameters should be tuned according to the criteria given in Appendix B. 


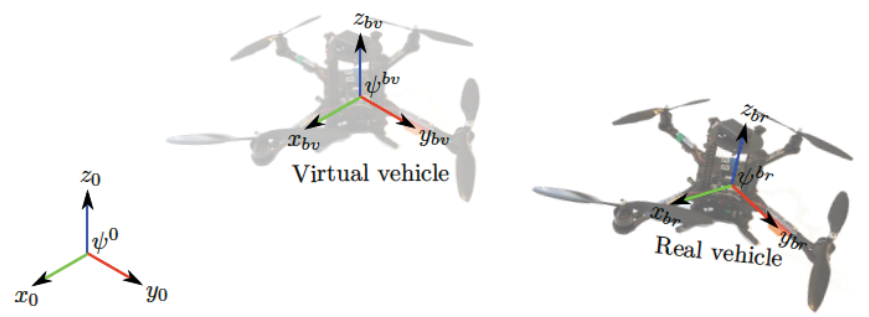

Figure 2.4: Inertial coordinate frame and the body fixed coordinate frames of the virtual and the real vehicles, attached at their respective center of mass.

In the proposed control architecture, every action of the virtual vehicle is associated to an energetic cost. The coupling of the virtual vehicle with both the operator and the real slave is variable. It can vary from full coupling to complete decoupling depending on the availability of energy in a storage, as introduced in the next section. As a consequence, the coupling wrench $\bar{W}_{b v-c}^{b v}$ is applied only if there is energy available in the storage and is given by

$$
\bar{W}_{b v-c}^{b v}=\bar{W}_{b v-v c}^{b v}+\bar{W}_{b v-r c}^{b v}
$$

where $\bar{W}_{b v-v c}^{b v}$ and $\bar{W}_{b v-r c}^{b v}$ are the wrenches due to the virtual slave's local controller and the viscoelastic coupling. Note that $\bar{W}_{b v}^{b v}$ is the modified version of $\bar{W}_{b v-c}^{b v}$ depending on the availability of energy in the local energy tank.

\subsubsection{Energy Tanks}

The concept of monitoring the energy of the system in bilateral teleoperation has been introduced in [109] and [30]. The idea has been formalized and the use of energy tanks has been proposed in [23]. In energy-based haptic teleoperation, actions that ensure the passivity of the system are taken by monitoring the energetic state of the system.

The energy tanks are sources of energy for every action on the virtual vehicle. Depending on the purpose that the tank is used for, two types of energy tanks are identified, namely, primary energy tank and backup energy tank.

The primary energy tank directly interacts with the virtual vehicle, making it the primary source of energy for the virtual vehicle. It has multistates that not only enable the enforcement of passivity, but also controllability of each DOF of the virtual vehicle [69]. The number of states of this tank is equal to the number of DOFs of the virtual vehicle.

The backup energy tank, on the other hand, is source of additional energy for the primary energy tank. It supplies/withdraws energy to/from the primary energy tank based on its current content in relation to the energy cost of the task performed on the virtual vehicle by the operator. For instance, if the operator needs to maneuver the vehicle with a higher velocity that requires higher energy than originally stored in the primary energy tank, the additional energy is supplied from the backup energy tank. To provide more insight and facilitate the design process, the tanks are modeled as dynamical systems. 


\subsubsection{Primary Energy Tank}

The primary energy tank is modeled as a two-port multidimensional $\mathbb{C}$-type storage element. The tank can be considered as an ideal variable spring whose rest-length $l$ and length $x$ can be changed over time. For ease of computation, consider a linear spring with a constant stiffness. The dynamics of this tank is then given by

$$
\left\{\begin{array}{l}
\left(\begin{array}{l}
\dot{x} \\
\dot{l}
\end{array}\right)=\left(\begin{array}{ll}
I & 0 \\
0 & I
\end{array}\right)\left(\begin{array}{l}
u_{x} \\
u_{l}
\end{array}\right) \\
\left(\begin{array}{l}
y_{x} \\
y_{l}
\end{array}\right)=\left(\begin{array}{ll}
I & 0 \\
0 & I
\end{array}\right)\left(\begin{array}{l}
\frac{\partial H(x, l)}{\partial x} \\
\frac{\partial H(x, l)}{\partial l}
\end{array}\right)
\end{array}\right.
$$

where $H(x, l)=\frac{1}{2}(x-l)^{T} K_{t}(x-l)$, in which $K_{t}$ is a $4 \times 4$ positive definite stiffness matrix; $\left(u_{x}, y_{x}\right)$ and $\left(u_{l}, y_{l}\right)$ are the length and the rest-length port variables, through which the tank interact with the virtual vehicle and the backup energy tank, respectively.

The ultimate goal of the interaction between the virtual vehicle and the primary energy tank is to enforce the desired behavior ${ }^{1}$ of the virtual vehicle without violating passivity, which is enforced by monitoring the states of the primary energy tank. To achieve this, the coupling between both the operator and the real vehicle with the virtual vehicle is modulated. This modulated coupling is regulated by the passivity-enforcing supervisor. The supervisor essentially defines the coupled dynamics of the primary energy tank and the virtual vehicle. The coupling wrench $\bar{W}_{b v-c}^{b v}$ in (2.3) modulates the power flow between them. The interconnection dynamics between the two is given by

$$
\left(\begin{array}{c}
\bar{W}_{b v}^{b v} \\
u_{x}
\end{array}\right)=\left(\begin{array}{cc}
0 & \Omega \\
-\Omega^{T} & 0
\end{array}\right)\left(\begin{array}{c}
\bar{T}_{b v}^{b v, 0} \\
y_{x}
\end{array}\right)
$$

where $\Omega=f\left(\bar{W}_{b v-c}^{b v}\right)$ is a diagonal matrix, which is a function of $\bar{W}_{b v-c}^{b v}$ and is assigned by the passivity-enforcing supervisor (see Fig. 2.3). An example on how this assignment of $\Omega$ can be performed is given in Section 2.9.2. Note that the supervisor does not modify $\bar{W}_{b v-c}^{b v}$ if there is enough energy in the primary tank, in that case $\bar{W}_{b v}^{b v}=\bar{W}_{b v-c}^{b v}$.

In bond graph, the coupled dynamics given in (2.5) is represented by the interaction of the primary energy tank $(\mathbb{C})$ and the virtual vehicle $(\mathbb{I}, \mathbb{M} \mathbb{G} \mathbb{Y})$ through the modulated transformer (MTFF), as shown in Fig. 2.5.

\subsubsection{Backup Energy Tank}

The backup energy tank is modeled as a single port 1-D I-type storage element. It is employed in the control structure with the aim of varying the energy content of the primary energy tank, with an ultimate goal of accomplishing various tasks using the virtual vehicle.

\footnotetext{
${ }^{1}$ The desired behavior is enforced by the resultant wrench $W_{b v-c}^{b v}$ of the local controller and the viscoelastic coupling.
} 


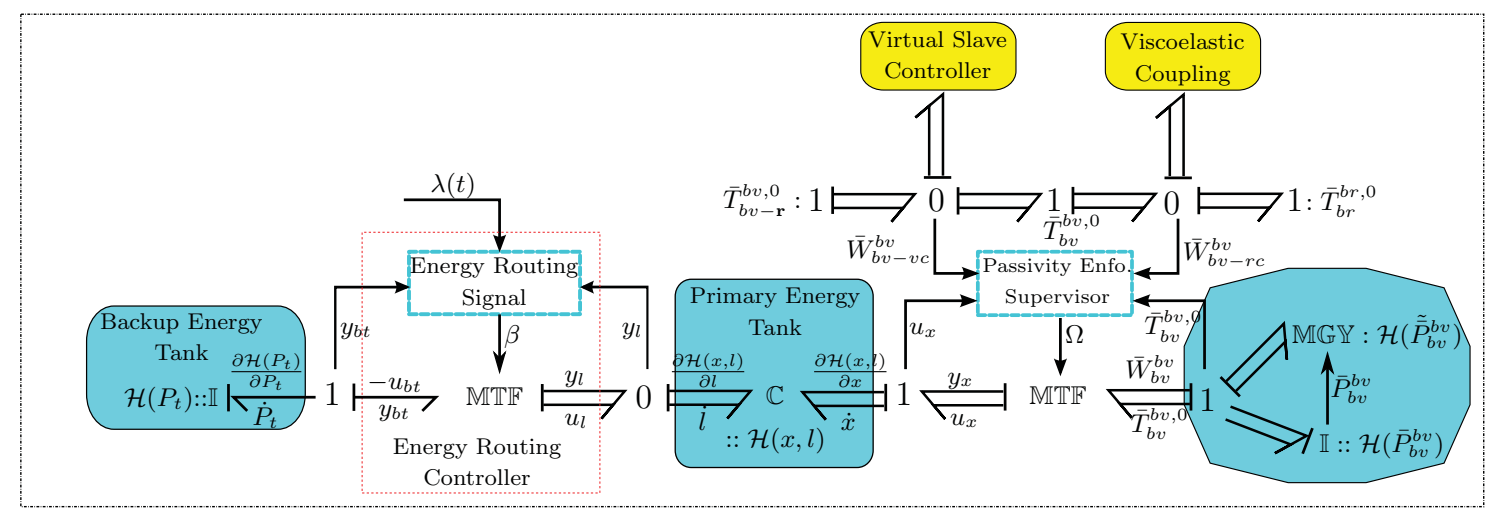

Figure 2.5: Bond graph model of the complete virtual slave system. The primary energy tank $(\mathbb{C})$ has two multidimensional ports through which it interacts with the backup energy tank, (II), and the virtual vehicle (I and $\mathbb{M} \mathbb{G} \mathbb{Y}$ ). The power conserving interactions among them are represented by two $\mathbb{M T T}$ s.

The dynamics of this backup energy tank is described as

$$
\left\{\begin{array}{l}
\dot{P}_{t}=u_{b t} \\
y_{b t}=\frac{\partial H\left(P_{t}\right)}{\partial P_{t}}
\end{array}\right.
$$

where $\left(u_{b t}, y_{b t}\right)$ are the port variables and $H\left(P_{t}\right)=\frac{1}{2 m_{t}} P_{t}^{2}$, in which $m_{t}$ is the inertia of the tank.

The backup tank trades energy with the primary tank with the goal of varying the energy content of the primary tank that is deemed necessary for the task executed by the operator. The primary tank interacts with the backup energy tank through its rest-length port. Among the possible ways to accomplish the desired energy trading in an energetically consistent way, a simple closed-loop energy routing controller is employed. The interaction of the two energy tanks through the energy routing controller is given by

$$
\left(\begin{array}{c}
u_{l} \\
u_{b t}
\end{array}\right)=\left(\begin{array}{cc}
0 & \beta \\
-\beta^{T} & 0
\end{array}\right)\left(\begin{array}{c}
y_{l} \\
y_{b t}
\end{array}\right)
$$

where $\beta=\eta y_{l} y_{b t}^{T}$ is a 4 -D vector, in which $\eta$ is a $4 \times 4$ matrix whose entries determine the magnitude and the direction of the power flow between the two tanks.

A way to achieve the energy trading between the two tanks is to associate the entries of $\eta$ to the desired rest-length $l_{d}$ of the primary tank. To that end, $\eta$ can be chosen as a diagonal matrix, i.e., $\eta=\operatorname{diag}\left\{l-l_{d}\right\} . l_{d}$ can be assigned based on the energy required to accomplish a specific task. In this study, it is associated with the mapping scale, as explained in Section 2.6.1. Clearly, the energy flow between the two tanks is blocked when $\beta=0$, i.e., at least one of $\eta, y_{l}, y_{b t}$ is zero. The blockage that occurs when $\eta=0$ is a desired control objective. However, in other cases, once it occurs, recovering from it would be impossible (i.e., a deadlock occurs). Nevertheless, lower bounding the primary energy tank and nonzero initialization of the backup energy tank prevent this deadlock. 


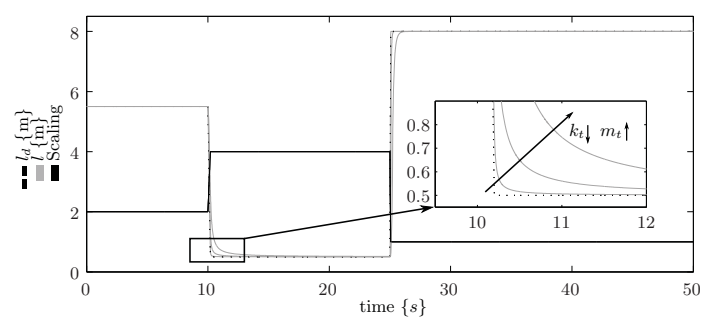

(a) Scaling factor, the desired and actual restlength of the primary energy tank.

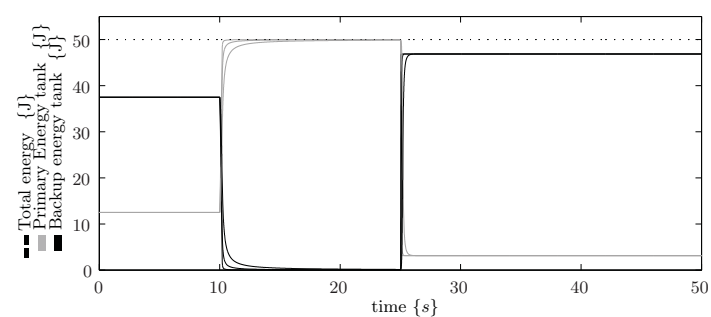

(b) Energies of the primary and backup energy tanks, and their combined energy.

Figure 2.6: Result illustrating the working principle of the interaction between the primary and backup energy tanks.

In Fig. 2.5, the energy routing controller is modeled as an $\mathbb{M T F}$, through which the primary energy tank, a 2-port multidimensional $\mathbb{C}$ element, interacts with the backup energy tank, a 1-port one dimensional $\mathbb{I}$ element.

Remark 2.1 The choice of modeling the primary and backup energy tanks as $\mathbb{C}$ and $\mathbb{I}$ elements is not unique, but rather for better intuition. Each energy tank can be modeled as either $\mathbb{C}$ or $\mathbb{I}$ type storage element as long as the interconnection dynamics with other elements are taken care of accordingly.

Remark 2.2 Similar result can also be achieved by merging the two tanks into one that carries out the responsibilities of the two. However, such distinction is chosen to elegantly present their functions from conceptual point of view.

Fig. 2.6 shows an example of the interaction between the primary and backup energy tanks. For this particular example, only one state of the primary energy is considered and the interaction between the virtual slave and the primary energy tank is blocked. In Fig. 2.6a, it is depicted that the desired rest-length $l_{d}$ is followed by $l$ of the primary energy tank according to the scaling factor (see also Section 2.6.1). Although $l \approx l_{d}$ by the action of the energy routing controller (provided that there is enough energy in the backup energy tank), the rate of convergence depends on $m_{t}^{-1} K_{t}$. The figure also shows the different rate of convergence for different values of $m_{t}^{-1} K_{t}$, i.e., the higher $m_{t}^{-1} K_{t}$, the faster the convergence becomes.

Fig. 2.6b shows the associated energy exchange between the two tanks. By varying $l$, only the required amount energy is presented in the primary tank, leading to a better performance. Observe that, irrespective of how the parameters of the two tanks are tuned, the total energy content of the two tanks remains constant. Hence, the interaction is passive.

\subsubsection{Virtual Slave Controller}

The virtual slave controller can be any type of controller that generates appropriate control inputs to accomplish the task commanded by the operator. The task considered in this work is state (pose and velocity) trajectory tracking. 
Let $\delta_{b v}^{0}=\left[\begin{array}{llll}\theta_{b v}^{0} & p_{b v_{x}}^{0} & p_{b v_{y}}^{0} & p_{b v_{z}}^{0}\end{array}\right]$ be the minimal representation of the pose of $\psi^{b v}$. An example of the dynamics of the trajectory tracking controllers can be given by

$$
\bar{W}_{b v-v c}^{b v}=\left\{\begin{array}{l}
\text { velocity controller } \\
-R_{v}\left(\bar{T}_{b v}^{b v, 0}-\bar{T}_{b v-r}^{b v, 0}\right) \\
\text { pose controller } \\
-\left(\bar{Z}_{b v}^{0}\right)^{T} K_{p}\left(\delta_{b v}^{0}-\delta_{b v-r}^{0}\right)-R_{p}\left(\bar{T}_{b v}^{b v, 0}-\bar{T}_{b v-r}^{b v, 0}\right)
\end{array}\right.
$$

where $\bar{Z}_{b v}^{0}$ is a transformation matrix, such that $\dot{\delta}_{b v}=\bar{Z}_{b v}^{0} \bar{T}_{b v}^{b v, 0}$ holds; $R_{v}, R_{p}, K_{p}$ are $4 \times 4$ positive definite matrices denoting the control parameters. The subscript $r$ denotes the desired reference state.

As shown in Section 2.7, any type of implementation of this controller does not compromise the passivity of the virtual slave system. This is due to the specific way by which the coupling of the virtual vehicle with the operator is implemented through the primary energy tank. Note, however, the performance is influenced by the type of the implemented controller.

\subsection{Real Slave System}

The real slave system is composed of the underactuated UAV and its controller. The VTOL UAVs, considered in this study are characterized by fully actuated rotational dynamics and underactuated translational dynamics. Only the translational DOF along the $z$-axis of the body fixed frame of the real vehicle $\psi^{b r}$ is actuated. Though the proposed tele-control algorithm works in a plug-and-play fashion with any type of VTOL UAVs [91], [16], [80] and their controllers [80], [37], [66], for completeness, a rough overview of an example of a real slave controller is provided hereunder.

The complete dynamics of the real slave is described by

$$
\left\{\begin{aligned}
\dot{P}_{b r}^{b r} & =\widetilde{P}_{b r}^{b r} \frac{\partial H\left(P_{b r}^{b r}\right)}{\partial P_{b r}^{b r}}+W_{b r}^{b r} \\
T_{b r}^{b r, 0} & =\frac{\partial H\left(P_{b r}^{b r}\right)}{\partial P_{b r}^{b r}}
\end{aligned}\right.
$$

where $H\left(P_{b r}^{b r}\right)=\frac{1}{2}\left(P_{b r}^{b r}\right)^{T}\left(I_{b r}^{b r}\right)^{-1} P_{b r}^{b r} ; P_{b r}^{b r}, T_{b r}^{b r, 0}$ and $W_{b r}^{b r}$ are vectors of dimension 6 , representing the generalized screw momenta, twist and wrench, respectively, of the real UAV.

$$
\widetilde{P}_{b r}^{b r}=\left[\begin{array}{cc}
\widetilde{P}_{b r-\omega}^{b r} & \widetilde{P}_{b r-v}^{b r} \\
\widetilde{P}_{b r-v}^{b r} & 0
\end{array}\right]
$$

is a block-wise skew symmetric matrix, consisting the first $\left(\bar{P}_{b r-\omega}^{b r}\right)$ and the last $\left(\bar{P}_{b r-v}^{b r}\right)$ three elements of $P_{b r}^{b r}$.

$W_{b r}^{b r}$ in (2.8) is the resultant wrench acting on the real UAV that gives the controlled real slave system a desired behavior. This is provided by the action of the controller.

$$
W_{\mathrm{cont}}^{b r}=W_{b r}^{b r}-W_{\mathrm{comp}}^{b r}
$$


where $W_{\text {comp }}^{b r}$ collects all wrenches acting on the UAV that need to be compensated by the controller $W_{\text {cont }}^{b r}$, see Appendix A. The compensated wrenches include gravity, friction, and aerodynamics. In free flight, for example, the main goal of the action of $W_{\text {cont }}^{b r}$ is often to track desired trajectory and/or avoid potential obstacles.

The final step in the control design is to allocate $W_{\text {cont }}^{b r}$ to the actuator inputs $\chi$. Depending on the actuation strategy of the real slave, $\chi$ can contain, for example, speed of propellers in quadrotors, thrust and AOA of controller vanes in ductedfans, and swash-plate angles in coaxial rotor-crafts [66]. The generalized mapping of $W_{\text {cont }}^{b r}$ to $\chi$ can be given by a function $\varrho($.$) , i.e.,$

$$
\chi=\varrho\left(W_{\text {cont }}^{b r}\right)
$$

The wrenches $\bar{W}_{b v-r c}^{b v}$ and $W_{b r}^{b r}$ are related by

$$
\bar{W}_{b v-r c}^{b v} \approx-\left(A d_{H_{b v}^{b r}}^{T} W_{b r}^{b r}\right)[3: 6]
$$

where $A d_{H_{b v}^{b r}}$ is the adjoint map used to transform coordinates with respect to which wrenches are expressed, and [3:6] represents the last four components of the 6-D vector.

\subsection{Master System}

The master is a device with a force displaying capability by which the operator interacts with the slave system. It is often characterized by having fully actuated DOFs and limited workspace. In the next sections, mapping and control methods that address issues attributed to using master devices for haptic teleoperation of aerial robots are proposed.

\subsubsection{Mapping}

To address the workspace limitation of the master and maximize the achievable precision, a switching-based variable scaled mapping of the pose of the master to the desired state reference of the slave, either velocity or pose, is proposed. When precision is of an essence, the pose of the master is mapped to a desired pose of the slave, hereafter referred as pose mode. When maneuvering in a wide workspace is desired, the pose of the master is mapped to a possibly scaled velocity reference of the slave, hereafter called rate mode. Such a mapping strategy, though for different applications, have been proposed in [33], [18], where the switching is dictated by the state of the slave, and not by the operator as proposed here. Besides, essential factors like time delay and stability have not been addressed, making the scheme less applicable in a wide range of applications.

In the proposed mapping algorithm, besides the switching between the two modes, the scaling factor can be changed online. Moreover, time delays that necessitate alternative interpretations of the state of the UAV at the time of switching are also considered, providing more flexibility to the strategy. 


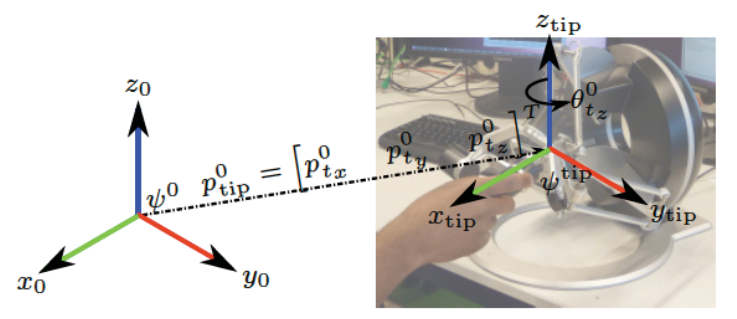

Figure 2.7: Inertial frame, $\psi^{0}$, and the master frame, $\psi^{\text {tip }}$.

It is intuitive to map the pose of the tip of the master device to the reference state of the slave to be controlled. Let the minimal and homogeneous matrix representations of the pose of the tip of the master device be $\delta_{m}=\left[\begin{array}{llll}\theta_{t_{z}}^{0} & p_{t_{x}}^{0} & p_{t_{y}}^{0} & p_{t_{z}}^{0}\end{array}\right]$, and $H_{\text {tip }}^{0}\left(\delta_{m}\right)$, respectively (see Fig. 2.7). Disregarding the rotation of the tip around the $x$ and $y$ axes $^{2}$ due to the underactuation of the slave, $H_{\mathrm{tip}}^{0}\left(\delta_{m}\right)$ has the following form

$$
H_{\text {tip }}^{0}\left(\delta_{m}\right)=\left[\begin{array}{cc}
\mathbf{R}_{\text {tip }}^{0} & p_{\text {tip }}^{0} \\
0 & 1
\end{array}\right]=\left[\begin{array}{cccc}
c\left(\theta_{t_{z}}\right) & -s\left(\theta_{t_{z}}\right) & 0 & p_{t_{x}}^{0} \\
s\left(\theta_{t_{z}}\right) & c\left(\theta_{t_{z}}\right) & 0 & p_{t_{y}}^{0} \\
0 & 0 & 1 & p_{t_{z}}^{0} \\
0 & 0 & 0 & 1
\end{array}\right]
$$

where $\theta_{t_{z}}$ is the rotation around the $z$ axis; $c($.$) and s($.$) represent the cosine and$ sine functions.

The mapping strategy, represented by a nonlinear function $\phi\left(H_{\mathrm{tip}}^{0}\right)$, provides a solution to bridge the gap between the two approaches that have conflicting design requirements to address the workspace and precision-related challenges. In pose mode, $H_{\text {tip }}^{0}$ is mapped to $H_{b v-r}^{0}$, and in rate mode to $\bar{T}_{b v-r}^{b v}$.

$$
\phi\left(H_{\text {tip }}^{0}\right):= \begin{cases}\phi_{\text {pose }}\left(H_{\text {tip }}^{0}\right)=H_{b v-r}^{0} & \text { pose mode } \\ \phi_{\text {rate }}\left(H_{\text {tip }}^{0}\right)=\bar{T}_{b v-r}^{b v, 0} & \text { rate mode }\end{cases}
$$

\subsubsection{Pose Mode}

The pose mode is suitable for achieving better precision while teleoperating in a limited workspace. In this mode, the pose of the tip of the haptic interface is mapped to a scaled pose reference of the virtual vehicle, thereby to the real slave due to the viscoelastic coupling. The mapping function is given by

$$
H_{b v-r}^{0}=\phi_{\text {pose }}\left(H_{\text {tip }}^{0}\right)=\left[\begin{array}{cc}
\mathbf{R}_{b v-r}^{0} & p_{b v-r}^{0} \\
0 & 1
\end{array}\right]
$$

with

$$
\begin{aligned}
\mathbf{R}_{b v-r}^{0} & =\left[\begin{array}{ccc}
c\left(\lambda_{\theta}(t) \theta_{t_{z}}+\alpha_{\theta}\right) & -s\left(\lambda_{\theta}(t) \theta_{t_{z}}+\alpha_{\theta}\right) & 0 \\
s\left(\lambda_{\theta}(t) \theta_{t_{z}}+\alpha_{\theta}\right) & c\left(\lambda_{\theta}(t) \theta_{t_{z}}+\alpha_{\theta}\right) & 0 \\
0 & 0 & 1
\end{array}\right] \\
p_{b v-r}^{0} & =\left[\begin{array}{ccc}
\lambda_{x}(t) p_{t_{x}}^{0}+\alpha_{x} & \lambda_{y}(t) p_{t_{y}}^{0}+\alpha_{y} & \lambda_{z}(t) p_{t_{z}}^{0}+\alpha_{z}
\end{array}\right]
\end{aligned}
$$

\footnotetext{
${ }^{2}$ Without loss of generality, $\psi^{\text {tip }}$ can be considered as having the same orientation around $x$ and $y$ axes as $\psi^{0}$.
} 
where $\lambda=\left[\begin{array}{llll}\lambda_{\theta} & \lambda_{x} & \lambda_{y} & \lambda_{z}\end{array}\right]^{T}$ is the scaling factor and $\alpha=\left[\begin{array}{llll}\alpha_{\theta} & \alpha_{x} & \alpha_{y} & \alpha_{z}\end{array}\right]^{T}$ is a vector representing the desired offset between the pose of the haptic interface and the slave.

In cases where the operator starts teleoperating in this mode at a neutral (calibration) pose of the haptic interface (i.e., $\psi^{\text {tip }}=\psi^{0}$ ),

$$
\alpha=\left[\begin{array}{llll}
\theta_{b v}\left(t_{s}-\tau_{s}\right) & p_{b v_{x}}^{0}\left(t_{s}-\tau_{s}\right) & p_{b v_{y}}^{0}\left(t_{s}-\tau_{s}\right) & p_{b v_{z}}^{0}\left(t_{s}-\tau_{s}\right)
\end{array}\right]^{T},
$$

i.e., the desired offset between the master and the slave is the pose of the slave perceived by the operator at the time of switching. $t_{s}$ and $\tau_{s}$ denote the time of switching and the last backward time delay just before switching, respectively.

However, when the pose mode is started at a nonneutral pose of the haptic device, two main mapping alternatives can be identified. The first alternative considers the pose of the slave, perceived by the operator at the time of switching, as a desired offset between the neutral pose of the master and the slave. This practically resets the center of the achievable workspace of the slave at the perceived pose of the slave at the time of switching. As a result, this submode is referred as reset. The other alternative, referred as the normal submode, considers the relative pose of the master and the slave at the time of switching as the desired offset between the current pose of the two. While the latter submode results in a smooth transition, it results in asymmetric teleoperating workspace with respect to the starting pose of the slave. The offset $\alpha$ in (2.13) in the two submodes is given by

$$
\alpha= \begin{cases}{\left[\begin{array}{l}
\theta_{b v}\left(t_{s}-\tau_{s}\right) \\
p_{b v}^{0}\left(t_{s}-\tau_{s}\right)
\end{array}\right],} & \text { reset } \\
{\left[\begin{array}{c}
\theta_{b v}\left(t_{s}-\tau_{s}\right)-\lambda_{\theta}\left(t_{s}\right) \theta_{t_{z}}\left(t_{s}\right) \\
p_{b v}^{0}\left(t_{s}-\tau_{s}\right)-\operatorname{diag}\left(\lambda_{x, y, z}\left(t_{s}\right)\right) p_{\text {tip }}^{0}\left(t_{s}\right)
\end{array}\right],} & \text { normal }\end{cases}
$$

\subsubsection{Rate Mode}

The rate mode provides the possibility of teleoperating the slave essentially in unlimited workspace using the limited stroke of the haptic interface. In this mode, the pose of the haptic interface is mapped to a scaled twist reference of the slave as follows:

$$
\bar{T}_{b v-r}^{b v, 0}=\phi_{\text {rate }}\left(H_{\text {tip }}^{0}\right)=\left[\begin{array}{c}
\lambda_{\theta}(t) \theta+\alpha_{\theta} \\
\mathbf{R}_{0}^{\operatorname{tip}} \operatorname{diag}\left(\lambda_{x, y, z}\left(t_{s}\right)\right) p_{\text {tip }}^{0}-\alpha_{x, y, z}
\end{array}\right]
$$

Similar to the pose mode, the offset $\alpha$ in the rate mode also provides two possible submodes of mapping when switching occurs at a nonneutral pose of the master device. In the normal submode, the pose at which the rate mode is started is considered as a new zero twist command to the slave, providing a less abrupt change in the twist reference of the slave compared with the reset submode. However, it results in asymmetric twist command, i.e., the maximum twists commanded in the both directions of an axis are different. The offset $\alpha$ in the two submodes is given 
by

$$
\alpha=\left\{\begin{array}{cc}
0, & \text { reset } \\
-\left[\begin{array}{c}
\lambda_{\theta}\left(t_{s}\right) \theta_{t_{z}}\left(t_{s}\right) \\
\mathbf{R}_{0}^{\mathrm{tip}}\left(t_{s}\right) \operatorname{diag}\left(\lambda_{x, y, z}\left(t_{s}\right)\right) p_{\text {tip }}^{0}\left(t_{s}\right)
\end{array}\right], & \text { normal }
\end{array}\right.
$$

Let $s$ be a binary logical input denoting the modes $(s=0$, pose mode and $s=1$, rate mode), then the generalized mapping function can be given by

$$
\phi\left(s, H_{\text {tip }}^{0}\right)=(1-s) \phi_{\text {pose }}\left(H_{\text {tip }}^{0}\right)+s \phi_{\text {rate }}\left(H_{\text {tip }}^{0}\right)
$$

The proposed mapping strategy is based on simple concepts of switching between intuitive modes and submodes. The switching is controlled by the operator and can be performed whenever necessary, providing more flexibility to the mapping algorithm. Moreover, it results in a better transparency since the operator is in control of the switching.

\subsubsection{Force Feedback}

As mentioned in Section 2.1.1, unlike classical teleoperation, in haptic teleoperation of UAVs during maneuvering tasks, the force feedback displayed to the operator is usually virtual. In this study, the haptic feedback that provides cues on the teleoperated state of the slave is proposed. Specifically, it is proportional to the deviation of the teleoperated state of the slave from the operator's command. Mathematically, it is

$$
\bar{W}_{m c}^{0}=-k_{F F}\left(\delta_{m}-\bar{\delta}_{m}\right)
$$

where $k_{F F}$ is a $4 \times 4$ stiffness matrix; the subscripts $m c$ refers to the master controller; $\bar{\delta}_{m}$ denotes the equivalent master pose of the controlled state of the slave; $\bar{\delta}_{m}$ is given by

$$
\begin{aligned}
\bar{\delta}_{m} & =\phi^{\#}\left(s, H_{b v}^{0}, \bar{T}_{b v}^{b v, 0}\right) \\
& =(1-s) \phi_{\text {pose }}^{\#}\left(H_{b v}^{0}\right)+s \phi_{\text {rate }}^{\#}\left(\bar{T}_{b v}^{b v, 0}\right)
\end{aligned}
$$

where $\phi^{\#}$ denotes the mapping function from the controlled state of the slave to the equivalent master pose. It is essentially the inverse of $\phi$.

\subsubsection{Variable Impedance Master Controller}

The main task of the master controller is generating the desired haptic feedback. However, to use the proposed control architecture in a wide range of operating conditions, additional considerations should be made. For instance, to change the force feedback sensitivity, one may need to adjust the stiffness matrix in (4.37). Such a process has energetic consequences that may compromise the passivity unless properly addressed. 
To increase the versatility of the proposed controller, the master controller is endowed with a variable spring and a local energy tank. All parameters of the spring (spring constant, rest-length and length) can be changed, provided that the energy required to complete the task can be made available from the master's energy tank and/or the operator.

Consider a variable spring with an energy function $H\left(k_{F F}, \Delta \delta_{m}, \delta_{l}\right)=H()=$. $\frac{1}{2}\left(\Delta \delta_{m}-\delta_{l}\right)^{T} k_{F F}\left(\Delta \delta_{m}-\delta_{l}\right)$, in which $\Delta \delta_{m}, \delta_{l}$ represent the length and the restlength, respectively. The dynamics of this spring is then given by

$$
\left\{\begin{array}{l}
\left(\begin{array}{c}
\Delta \dot{\delta}_{m} \\
\dot{\delta}_{l} \\
\dot{k}_{F F}
\end{array}\right)=\left(\begin{array}{llll}
I & I & 0 & 0 \\
0 & 0 & I & 0 \\
0 & 0 & 0 & I
\end{array}\right)\left(\begin{array}{c}
u_{\delta_{m}} \\
u_{\bar{\delta}_{m}} \\
u_{\delta_{l}} \\
u_{k_{F F}}
\end{array}\right) \\
\left(\begin{array}{c}
\bar{W}_{\delta_{m}}^{0} \\
\bar{W}_{\bar{\delta}_{m}}^{0} \\
\bar{W}_{\delta_{l}}^{0} \\
\bar{W}_{k_{F F}}^{0}
\end{array}\right)=\left(\begin{array}{lll}
I & 0 & 0 \\
I & 0 & 0 \\
0 & I & 0 \\
0 & 0 & I
\end{array}\right)\left(\begin{array}{l}
\frac{\partial H(.)}{\partial \Delta \delta_{m}} \\
\frac{\partial H(.)}{\partial \delta_{l}} \\
\frac{\partial H(.)}{\partial k_{F F}}
\end{array}\right)
\end{array}\right.
$$

where $u_{\delta_{m}}=\dot{\delta}_{m}$ and $u_{\bar{\delta}_{m}}=-\dot{\bar{\delta}}_{m}$.

The variable spring interacts with the master device and the master's energy tank with the first and the last three power ports, respectively. The power conserving energy consistent dynamic interaction of the spring and the tank is described by the interconnection

$$
\left(\begin{array}{c}
\dot{\Lambda} \\
u_{*}^{0}
\end{array}\right)=\left(\begin{array}{cc}
0 & \sigma \\
-\sigma^{T} & 0
\end{array}\right)\left(\begin{array}{c}
\frac{\partial H(\Lambda)}{\partial \Lambda} \\
W_{*}^{0}
\end{array}\right)
$$

where $\bar{W}_{*}^{0}=\left[\begin{array}{lll}\bar{W}_{\bar{\delta}_{m}}^{0} & \bar{W}_{\delta_{l}}^{0} & \bar{W}_{k_{F F}}^{0}\end{array}\right]^{T}$ and $u_{*}^{0}=\left[\begin{array}{lll}u_{\bar{\delta}_{m}} & u_{\delta_{l}} & u_{k_{F F}}\end{array}\right]^{T} ; H(\Lambda)$ and $\Lambda$ are the Hamiltonian and the state of the tank; $\sigma=\left[\begin{array}{lll}\sigma_{\bar{\delta}_{m}} & \sigma_{\delta_{l}} & \sigma_{k_{F F}}\end{array}\right]$ is a transformation vector.

In (2.21), $\sigma$ is a function of the current and desired states of the spring and the energy tank. It is assigned based on the desired controller behavior for the task. In the considered application for instance, the tasks may include varying the stiffness of the spring to change the sensitivity of the haptic feeling, changing the length of the spring to generate force feedback and/or changing the rest-length to maintain the current position of the slave in the absence of the operator's input in pose mode.

Unlike the energy tanks of the virtual slave system, only initial prefilling of the master tank may not be enough to accomplish the desired tasks. This is primarily attributed to the inevitable energy leakage due to the inherent friction present in the haptic interface and possible dissipative effect of the operator. On the other hand, excessive prefiling of the tank to mitigate the depletion may result in undesired intermediate dynamic behaviors. Here, it is proposed to endow the master controller with virtual energy dissipating elements by which energy can be harvested. 
With reference to (2.1) (see also Fig. 2.2), the dissipating elements of the controller are $R_{1}$ and $R_{2} . R_{1}$ represents a dissipating element acting on the tangent space at the states of the controller $\Delta \delta_{m}, \delta_{l}$, and $k_{F F}$ (for simplicity only at $\Delta \delta_{m}$ is considered here), while $R_{2}$ indicates the one acting on the cotangent space at the input of the controller $\bar{u}_{m c}=u_{\delta_{m}}$. The harvesting strategy is to replenish the energy tank with the amount of energy that is virtually dissipated by $R_{1}$ and $R_{2}$. This can be accomplished by the interconnection dynamics of the damping elements and the master energy tank given by

$$
\left(\begin{array}{c}
\dot{\Lambda} \\
\bar{W}_{R}^{0}
\end{array}\right)=\left(\begin{array}{cc}
0 & \gamma_{m} \zeta \\
-\zeta^{T} & 0
\end{array}\right)\left(\begin{array}{c}
\frac{\partial H(\Lambda)}{\partial \Lambda} \\
u_{R}
\end{array}\right)
$$

where $\left(u_{R}, \bar{W}_{R}^{0}\right)$ is the port variables of the damping element(s) and $\zeta$ is a transformation vector. $\gamma_{m}$ is a binary logical inputs that permits/block the energy harvesting process.

As storing too much energy, however, may result in undesirable dynamics that may even lead to loss of passivity in the desired operating range, it is desirable to dissipate extra energy above a predefined upper bound. This is essentially performed by $\gamma_{m}$, i.e., when $\gamma_{m}=1$, energy is harvested, whereas the harvesting process is disabled when $\gamma_{m}=0$.

For better intuition, the energy replenishing process is explained by means of an example. Consider linear dissipative elements ${ }^{3}$ on both the state of the spring length $\Delta \delta_{m}$ and the input of the master controller $\bar{u}_{m c}$, i.e., $\left(u_{R}\right)^{T}=\left[\begin{array}{ll}\frac{\partial^{T} H(.)}{\partial \Delta \delta_{m}} & \bar{u}_{m c}^{T}\end{array}\right]$ and $\left(\bar{W}_{R}^{0}\right)^{T}=\left[\begin{array}{ll}-\left(R_{1} \frac{\partial^{T} H(.)}{\partial \Delta \delta_{m}}\right)^{T} & -\left(R_{2} \bar{u}_{m c}\right)^{T}\end{array}\right]$. The rate at which energy is dissipated is

$$
\left(\bar{W}_{R}^{0}\right)^{T} u_{R}=-\frac{\partial^{T} H(.)}{\partial \Delta \delta_{m}} R_{1} \frac{\partial H(.)}{\partial \Delta \delta_{m}}-\bar{u}_{m c}^{T} R_{2} \bar{u}_{m c}
$$

Now, the energy tank can be replenished by the same amount of energy that is virtually dissipated in these dissipating elements by choosing ${ }^{4}$

$$
\begin{aligned}
\zeta & =\left[\begin{array}{ll}
\zeta_{R_{1}} & \zeta_{R_{2}}
\end{array}\right] \\
& =\left[\begin{array}{ll}
\left(\frac{\partial H(\Lambda)}{\partial \Lambda}\right)^{-1} \frac{\partial^{T} H(.)}{\partial \Delta \delta_{m}} R_{1} & \left(\frac{\partial H(\Lambda)}{\partial \Lambda}\right)^{-1} \bar{u}_{m c}^{T} R_{2}
\end{array}\right]
\end{aligned}
$$

As it is shown in Section 2.7, with reference to (2.20)-(2.24) the master controller is transformed to a lossless system, provided that $\gamma_{m}=1$. In order not to compromise the fidelity of the haptic feedback due to the addition of damping elements, the force contribution of the damping elements can be saturated. In fact, it is possible to switch OFF the damping effect completely when there is too much energy in the energy tank. However, it may result in a continuous ON-OFF effect that could be distracting for the operator [23]. On the contrary, with the proposed saturated damping, one can accomplish the desired task passively and effectively.

\footnotetext{
${ }^{3}$ The parameters of $R_{1}$ and $R_{2}$ that are considered here are symmetric and positive definite matrices of appropriate dimensions.

4 To avoid numerical singularity while assigning $\zeta$, the energy tank of the master controller should be lower bounded by a small positive number.
} 


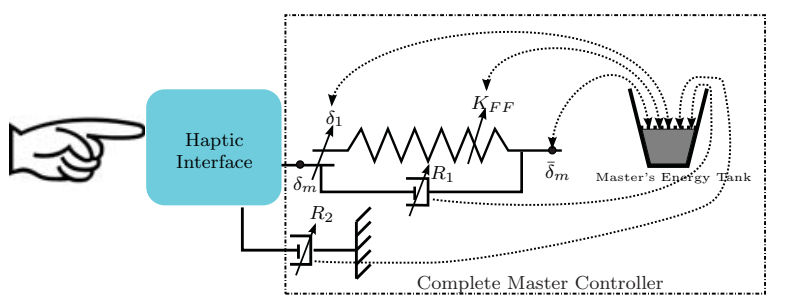

Figure 2.8: Iconic diagram representation of the variable impedance controller.

It may also be necessary to vary the damping parameters significantly. For instance, they can be increased to stabilize the haptic interface according to the change in the geometrical properties of the variable spring in the controller, when there is no operator input to counteract the haptic feedback. This would eventually result in stabilization of the slave even in the absence of the operator's input.

The complete dynamics of the variable impedance controller is obtained by combining (2.20)-(2.24) and is given by

$$
\left\{\begin{array}{l}
\left(\begin{array}{c}
\dot{r} \\
\dot{\Lambda}
\end{array}\right)=\left(\begin{array}{cc}
-\tilde{R}_{1} & -\sigma^{T} \\
\sigma+\gamma_{m} \tilde{\zeta}_{R_{1}} & 0
\end{array}\right)\left(\begin{array}{c}
\frac{\partial H(.)}{\partial r} \\
\frac{\partial H(\Lambda)}{\partial \Lambda}
\end{array}\right)+\left(\begin{array}{c}
\digamma \\
\gamma_{m} \zeta_{R_{2}}
\end{array}\right) \bar{u}_{m c} \\
\bar{W}_{m c}^{0}=\bar{W}_{\delta_{m}}^{0}=\left(\begin{array}{ll}
\digamma^{T} & 0
\end{array}\right)\left(\begin{array}{c}
\frac{\partial H(.)}{\partial r} \\
\frac{\partial H(\Lambda)}{\partial \Lambda}
\end{array}\right)+R_{2} \bar{u}_{m c}
\end{array}\right.
$$

where $r^{T}=\left[\begin{array}{lll}\bar{\delta}_{m} & \delta_{l} & k_{F F}\end{array}\right] ; \tilde{\zeta}_{R_{1}}=\left[\begin{array}{lll}\zeta_{R_{1}} & 0 & 0\end{array}\right] ; \tilde{R}_{1}=\operatorname{diag}\left(\begin{array}{lll}R_{1} & 0 & 0\end{array}\right) ;$ and $\digamma^{T}=$ $\left[\begin{array}{lll}I & 0 & 0\end{array}\right]$.

From (2.25), it can be seen that the modified haptic feedback displayed to the operator is

$$
\bar{W}_{m c}^{0}=k_{F F}\left(\Delta \delta_{m}-\delta_{l}\right)+R_{2} \bar{u}_{m c}
$$

Fig. 2.8 shows the iconic diagram representation of the proposed variable impedance master controller. Fig. 2.9 shows its equivalent representation in bond graphs. The variable spring is modeled as a multiport multidimensional $\mathbb{C}$-element. The energy tank, instead, is modeled as a single state single-port $\mathbb{C}$-element. The power conserving process of both energy replenishing, by using the virtual damping elements, and varying the geometric properties of the variable spring through the coupled dynamics described in (2.25), is captured by the modulated network structure $\mathcal{D}$.

\subsection{Passivity Analysis}

Many authors have used passivity as a desired control objective to design a stable teleoperation system. In rate-based teleoperation of an aerial vehicle, the interconnection between the master controller and the communication channel is not realized through the passive output (velocity), rather through its position. Hence, it is not possible to exploit passivity's invariance property during feedback interconnection to deduce coupled passivity. In rate control, a nonzero constant position 


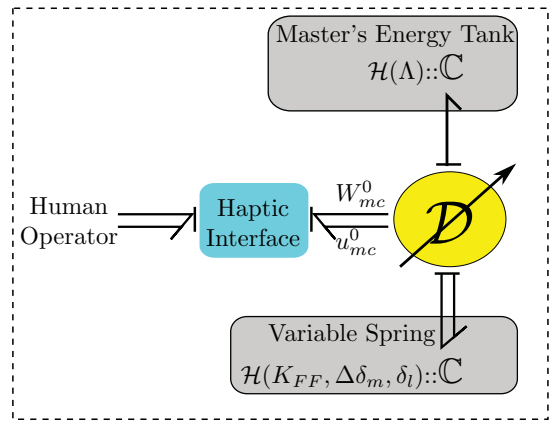

Figure 2.9: Bond graph equivalent of Fig. 2.8. D represents the variable network topology that describes the energy distribution among the elements of the controller. Note that $\left(W_{m c}^{0}\right)^{T}=$ $\left[\begin{array}{lll}0 & 0 & \left(\bar{W}_{m c}^{0}\right)^{T}\end{array}\right]$ and $u_{m c}^{T}=\left[\begin{array}{lll}0 & 0 & \bar{u}_{m c}^{T}\end{array}\right]$.

of the master represents a desired velocity of the slave. Considering the velocity of the master is zero, there is no energy flowing into the system from the human. However, this is not the case on the side of the slave, where motion with a possible force application would result in energy injection to the slave. This section discusses and provides mathematical proof on how this important consideration has been incorporated to achieve passivity of the complete system.

As explained in Sections 2.4 and 2.6, the virtual slave system and the master controller are designed to be passive. The energy tanks of both subsystems, along with their respective coupled dynamics, are paramount for the passiveness of each system. The fact that the passivity of each subsystem is enforced, as opposed to enforcing it on each component of each subsystem, relaxes the conservativeness, and therefore, a better performance can be achieved.

In the proposed algorithm, unlike other energy-based teleoperation algorithms [30], [24], only relevant signals, based upon which the local behaviors of each subsystems are varied, are exchanged across the communication channels. The additional merit of the localness of the energy circuits of each subsystem is that issues associated with energy packet exchange are avoided, such as, additional traffic in the communication network, difficulty of convergence of the energy tanks contents, and reduced performance that stems from the unavailability of the energy in the communication channel.

Assuming that the operator operates passively, the stability of the high-level teleoperation loop is guaranteed under any operating condition. The passivity of the complete virtual slave system and the master controller with respect to their total Hamiltonian, $H\left(\bar{P}_{b v}^{b v}, x, l, P_{t}\right)=H\left(\bar{P}_{b v}^{b v}\right)+H(x, l)+H\left(P_{t}\right)$ and $H\left(k_{F F}, \Delta \delta_{m}, \delta_{l}, \Lambda\right)$ $=H()+.H(\Lambda)$, and supply rate, $u^{T} y=\left(\bar{W}_{b v}^{b v}\right)^{T} \bar{T}_{b v}^{b v, 0}+u_{x}^{T} y_{x}+u_{l}^{T} y_{l}+u_{b t} y_{b t}$ and $u^{T} y=\bar{u}_{m c}^{T} \bar{W}_{m c}^{0}$, is shown as follows.

Proof: The Virtual Slave

$$
\begin{aligned}
\frac{\mathrm{d} H\left(\bar{P}_{b v}^{b v}, x, l, P_{t}\right)}{\mathrm{d} t} & =\frac{\partial H^{T}}{\partial \bar{P}_{b v}^{b v}} \dot{\bar{P}}_{b v}^{b v}+\frac{\partial H^{T}}{\partial x} \dot{x}+\frac{\partial H^{T}}{\partial l} \dot{l}+\frac{\partial H}{\partial P_{t}} \dot{P}_{t} \\
& =\frac{\partial H^{T}}{\partial \bar{P}_{b v}^{b v}} \tilde{\bar{P}}_{b v}^{b v} \frac{\partial H}{\partial \bar{P}_{b v}^{b v}}+\left(\bar{W}_{b v}^{b v}\right)^{T} \bar{T}_{b v}^{b v, 0}+u_{x}^{T} y_{x}+u_{l}^{T} y_{l}+u_{b t} y_{b t} \\
& =0+y_{x}^{T} \Omega^{T} \bar{T}_{b v}^{b v}, 0-\left(\bar{T}_{b v}^{b v, 0}\right)^{T} \Omega y_{x}+y_{b t} \beta^{T} y_{l}-y_{l}^{T} \beta y_{b t}=0
\end{aligned}
$$


Table 2.1: Parameters (in SI units)

\begin{tabular}{|c|c|}
\hline \multicolumn{2}{|c|}{ Quadrotor inertia parameters (experiments) } \\
\hline \multicolumn{2}{|c|}{$I_{b r}^{b r}=\operatorname{diag}(0.002,0.002,0.002,1.3,1.3,1.3)$} \\
\hline \multicolumn{2}{|c|}{ Ducted-fan inertia parameters (simulations) } \\
\hline \multicolumn{2}{|c|}{$I_{b r}^{b r}=\operatorname{diag}(0.018,0.018,0.018,1.5,1.5,1.5)$} \\
\hline Virtual slave and master parameters $\left(I_{4}\right.$ is a $4 \times 4$ identity matrix) \\
\hline$I_{b v}^{b v}=\operatorname{diag}(0.002,1.5,1.51 .5) 10^{-2}$ & $\lambda \in[5,15], \mu=0.1$ \\
\hline$k_{F F}=15 I_{4}, R_{v}=50 I_{4}$ & $K_{p}=100 I_{4}, R_{p}=50 I_{4}$ \\
\hline$k_{c}=150 I_{4}, d_{c}=200 I_{4}$ & $K_{t}=10 I_{4}, m_{t}=0.01$ \\
\hline
\end{tabular}

Clearly, the virtual slave system is a lossless passive system. The control signals due to the operator stimulus and the visco-elastic coupling with the real slave only affect the energy redistribution among the virtual vehicle, the primary and back-up energy tanks.

Proof: $\quad$ The Variable Impedance Master Controller

$$
\begin{aligned}
\frac{\mathrm{d} H(r, \Lambda)}{\mathrm{d} t} & =\frac{\partial H^{T}}{\partial r} \dot{r}+\frac{\partial H^{T}}{\partial \Lambda} \dot{\Lambda} \\
& =-\frac{\partial H^{T}}{\partial r} \tilde{R}_{1} \frac{\partial H}{\partial r}-\frac{\partial H^{T}}{\partial r} \sigma^{T} \frac{\partial H}{\partial \Lambda}+\frac{\partial H^{T}}{\partial r} \digamma \bar{u}_{m c}+\frac{\partial H^{T}}{\partial \Lambda}\left(\sigma+\tilde{\zeta}_{R_{1}}\right) \frac{\partial H}{\partial r}+\frac{\partial H^{T}}{\partial \Lambda} \zeta_{R_{2}} \bar{u}_{m c} \\
& =\left(-\frac{\partial H^{T}}{\partial r} \tilde{R}_{1}+\frac{\partial H^{T}}{\partial \Lambda} \tilde{\zeta}_{R_{1}}\right) \frac{\partial H}{\partial r}+\left(\frac{\partial H^{T}}{\partial r} \digamma+\frac{\partial H^{T}}{\partial \Lambda} \zeta_{R_{2}}\right) \bar{u}_{m c} \\
& =\left(\gamma_{m}-1\right)\left\{\frac{\partial^{T} H(\cdot)}{\partial \Delta \delta_{m}} R_{1} \frac{\partial H(.)}{\partial \Delta \delta_{m}}+\bar{u}_{m c}^{T} R_{2} \bar{u}_{m c}\right\}+\left(\bar{W}_{m c}^{0}\right)^{T} \bar{u}_{m c}
\end{aligned}
$$

Depending on $\gamma_{m} \in\{0,1\}$, the variable impedance master controller is either lossless or strictly passive.

Proof: $\quad$ The High-level Teleoperation Loop

The Hamiltonian of the high-level teleoperation loop is $H=H\left(\bar{P}_{b v}^{b v}, x, l, P_{t}\right)+$ $H\left(k_{F F}, \Delta \delta_{m}, \delta_{l}, \Lambda\right)$, and supply rate $\bar{u}_{m c}^{T} \bar{W}_{m c}^{0}$. As a direct consequence of the aforementioned two proves, the high-level teleoperation loop is passive under all operating conditions.

\subsection{Simulations}

Validating simulations have been run in 20sim (Controllab Products B.V., Enschede, The Netherlands), an energy based software package. In the simulations, a model of a ducted-fan miniature UAV [80] and a trajectory tracking controller, as proposed in [66], are used as a real slave and low-level controller. The operator is modeled as a PD controller on the desired position of the master. Besides, the practical considerations suggested in Section 2.9.2 are incorporated in the dynamic model used for the simulations. The model and control parameters used in the simulations are given in Table 2.1.

\subsubsection{Switching}

The first two simulations show the teleoperation of the UAV in different sub/modes with and without time delays. In both simulations, similar motion profile of the master is used for better comparison, and the UAV is teleoperated in pose mode 
$(0-15 \mathrm{~s})$; rate mode $(15-32 \mathrm{~s})$; pose mode, normal submode $(32-47 \mathrm{~s})$; rate mode, normal submode (47 - $62 \mathrm{~s})$; pose mode, reset submode $(62-77 \mathrm{~s})$ and rate mode, reset submode $(77-90 \mathrm{~s})$.

Fig. 2.10 shows the results of the first two simulations, in which plots with faded and dark lines are results with no time delay (i.e., $\tau=0 \mathrm{~s}$ ) and delay of $\tau=1.2 \mathrm{~s}$, respectively. As can be seen from the plots, the desired trajectory tracking task is performed successfully in all modes with and without time delay. Because of the viscoelastic coupling, the virtual vehicle is able to reliably convey the state of the real slave, enabling the operator to successfully teleoperate the real slave.

As explained in Section 2.6.1, Figs. 2.10c and 2.10d show that the normal submodes in both modes result in a less abrupt transition than the reset submode at the time of switching. This effect is also manifested in the force feedback displayed to the operator. The magnitude and force feedback shown in Fig. 2.10b provides a haptic cue to the operator based on how large and in which direction the UAV is deviating from the command. The magnitude of this force feedback generally gets larger as the time delay in the communication channel increases, which explains why the force feedback in simulation 2 is generally larger than in simulation 1 .

\subsubsection{Variable Scale Mapping}

In the next two simulations, the UAV is tele-controlled in rate mode under variable scale mapping in the presence of delay, $\tau=1.5 \mathrm{~s}$. During the operation, a wind gust of $10 \mathrm{~N}$ is applied along $z$ axis from $55-70 \mathrm{~s}$. Besides, in the third simulation, the operator releases the master device after $70 \mathrm{~s}$.

Figs. 2.11 and 2.12 show the results from the third (faded lines) and fourth (dark lines) simulations. In the first half of both simulations, periodic motion profile of the master (see Fig. 2.11a) is mapped to the velocity of the UAV (see Fig. 2.11c) scaled by $\lambda \in[5,15]$ (see Fig. 2.11a). In the simulations, the energy tanks are initialized based on the guideline presented in Section 2.9.2, i.e., the primary energy tank initially has the maximum kinetic energy that one would like the virtual vehicle to acquire at the initial scaling factor (in the simulations $\lambda(0)=10)$, and the backup tank has the extra energy required while operating using the maximum scaling factor $(\bar{\lambda}=15)$. While the total energy of the complete virtual slave system remains the same (see Fig. 2.12a), the energy is rerouted among the energetic elements depending upon $\lambda$ and $\Omega$ assigned by the supervisor. When $\lambda$ changes, the energy distribution of the primary and backup energy tanks is also changed accordingly by modifying the rest-length of the primary tank and the momentum of the backup tank (see Fig. 2.12b). Similarly, energy is rerouted between the virtual vehicle and the primary energy tank by modifying the length of the primary tank (see Fig. 2.12c) when $\Omega$ changes.

The additional merit of the multi-state energy tank is clearly seen when the wind gust is applied along the $z$ axis. Even if the gust is strong enough to move the real UAV with a velocity more than the maximum allowed for the virtual vehicle, it does not influence the motion along the other axes even in the presence of adequate energy in the primary energy tank. This is due to the fact that passivity is enforced 


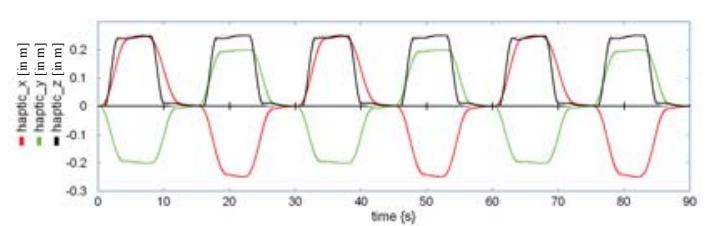

(a) Master position

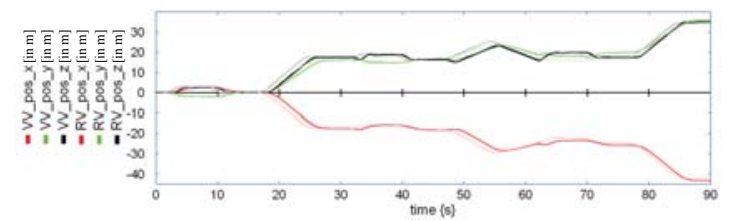

(c) Virtual and real UAV positions

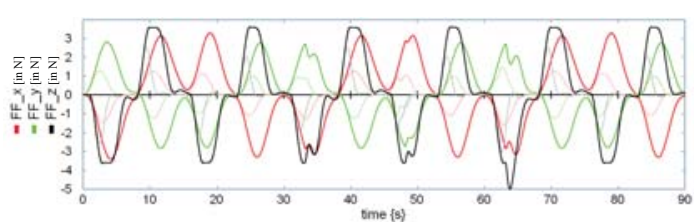

(b) Force feedback

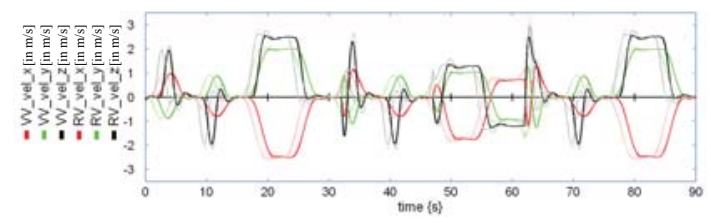

(d) Virtual and real UAV velocities

Figure 2.10: Results of the first $(\tau=0)$ and second $(\tau=1.2)$ simulations, presented by the faded and dark lines, respectively. In plots $2.10 \mathrm{c}$ and $2.10 \mathrm{~d}$, the dashed and solid lines are states of the real and virtual UAVs, respectively.

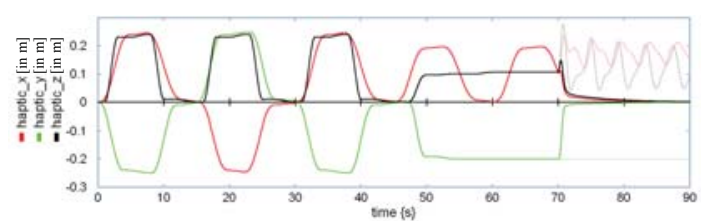

(a) Master positions.

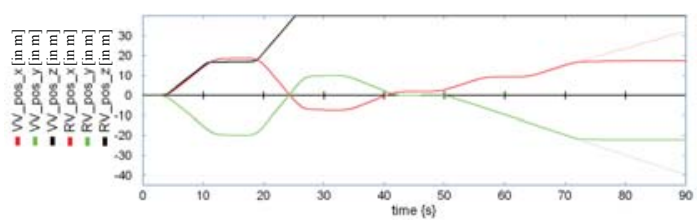

(c) Virtual and real UAV positions.

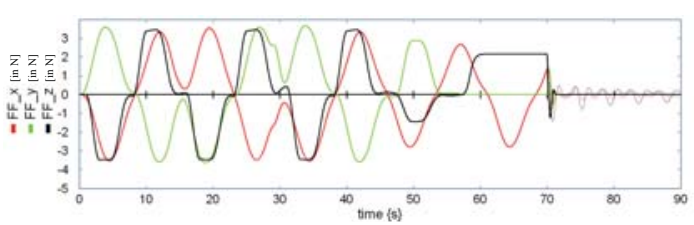

(b) Force feedbacks.

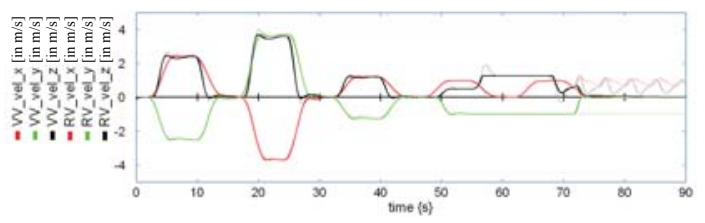

(d) Virtual and real UAV velocities.

Figure 2.11: Results of the third and fourth simulations (both with $\tau=1.5 \mathrm{~s}$ ), presented by the faded and dark lines, respectively. In plots $2.11 \mathrm{c}$ and $2.11 \mathrm{~d}$, the dashed and solid lines are states of the real and virtual UAVs, respectively.

not only based on the total energy but also on the energy allocated for each DOF, which makes the complete system more controllable. Note that because of the viscoelastic coupling, the real slave's velocity is brought back to the allowable limit after a short deviation due to the wind gust.

In the final phase of both simulations, the operator releases the handle of the master device. In simulation 3, because of the nonzero force feedback acting on the master device (see Fig. 2.11b), it keeps on moving differently. So does the slave since the pose of the master is in turn mapped to the controlled state of the slave (the velocity in this case). This unintended effect can still be present with no force feedback as long as the master device is displaced from its neutral pose or if a disturbance acts on the slave. However, as shown in the results of simulation 4, by varying the parameters of the variable impedance master controller (the damping, stiffness and length of the spring), the master is brought to its neutral pose and the slave starts hovering (see Fig. 2.11c). 


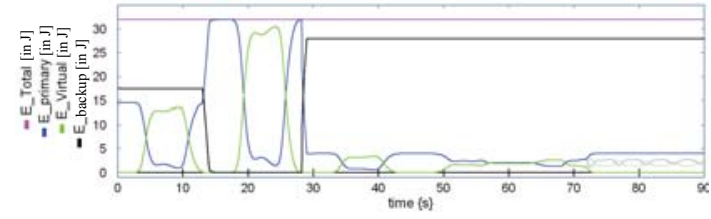

(a) Total energy of the complete virtual slave system, energy content of the primary and backup tanks and the virtual vehicle.

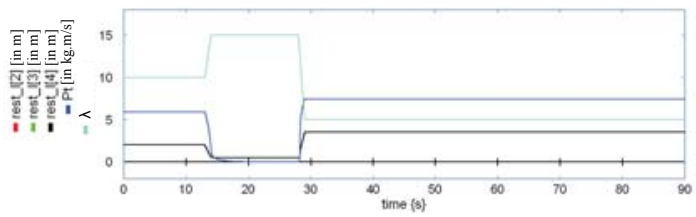

(b) Rest-length of the primary energy tank, momentum of the backup energy tank and the scaling factor.

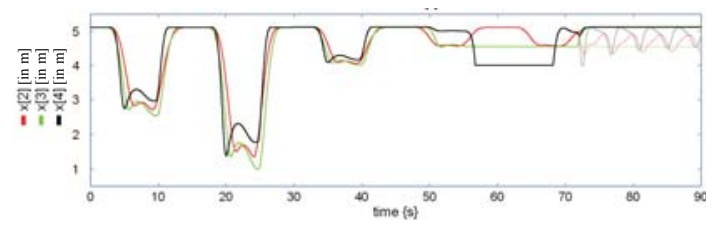

(c) The length of the primary energy tank.

Figure 2.12: Energy contents and the states of the primary, backup energy tanks and the virtual vehicle, and the mapping scale.

\section{$2.9 \quad$ Experiments}

In this section, the experimental setup used to demonstrate the applicability of the algorithm, the most important practical considerations and experimental results illustrating the effectiveness of the proposed control architecture are presented.

\subsubsection{Experimental Setup}

As shown in Fig. 2.13, the hardware architecture of the test setup consists of the ForceDimension Omega6 (Force Dimension, Nyon, Switzerland) haptic interface, the Asctec Pelican quadrotor (Ascending Technologies GmbH, Krailling, Germany), the OptiTrack (NaturalPoint, Inc. Corvallis, OR, USA) external tracking system and a base station. The Omega6 is characterized by high precision position and orientation sensing, 3 DOFs force displaying capability and active gravity compensation. The Asctec Pelican quadrotor is equipped with IMU sensors, ARM7 microprocessors and a Flight Control Unit. The pose of the UAV is estimated using the OptiTrack motion capture system, based on the data from passive markers attached on the vehicle. The ground station consists of two computers running Linux and Windows7 operating systems, in which the high-level teleoperation controller and pose estimation are running, respectively.

For its modularity, flexibility and expandability, the software environment is mainly based on the ROS. Various packages necessary for proper implementation of the proposed algorithm have been partly developed and others already available have been used with modification. In addition, for fast prototyping Matlab (MathWorks, Natick, MA, USA) has also been incorporated in the software architecture. For communication between the base station and the quadrotor, a User Datagram Protocol (UDP) has been used. 


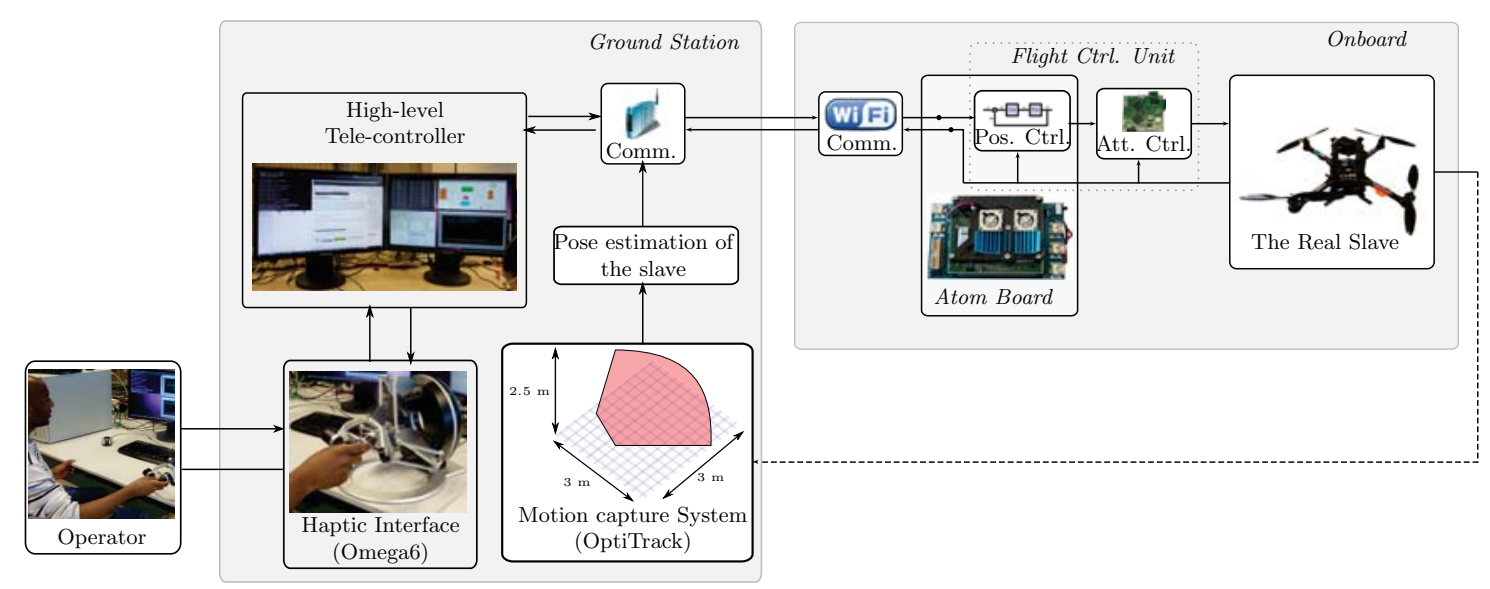

Figure 2.13: The complete experimental setup is the result of a system integration of various hardware and software. It consists of the operator interacting with the complete teleoperation system through the omega6 haptic interface, which is connected to the base station PC. Data is exchanged between the base station and the atom board of the pelican through a wifi connection using UDP protocol. The rigid pose of the pelican is estimated by the OptiTrack.

\subsubsection{Practical Considerations}

For proper realization of the proposed tele-control algorithm and maximization of its effectiveness, various practical considerations should be taken into account. Some of the most important ones are briefly presented hereunder.

Mode Switching and Scaling: The mapping algorithm is flexible enough that it is possible to change the mode of operation of each DOF of the slave and their mapping scale individually. Nevertheless, from implementation viewpoint, it requires different mechanism (either software or hardware) to switch between the modes and change the mapping scale individually. But more importantly, doing so results in high cognitive overload and eventually, deterioration of performance. Hence, switching the modes and the scaling altogether at the same time is largely recommended for task effectiveness.

Haptic Interface Calibration: Most haptic interfaces, currently existing on the market, need to be calibrated before employing them for teleoperation. In most applications it is desirable to choose the zero configuration of the haptic interface at the center of its workspace so that symmetric mapping is possible in both directions of the axis of each DOF. Moreover, as detecting the zero pose of the haptic interface may be important during operation, particularly when switching between modes, creating a small dead zone around the zero pose in its workspace is necessary. Additionally, such dead zone provides a certain degree of decoupling between highly coupled translational DOFs of commercially available haptic interfaces with parallel structure.

Scaling Factor $\lambda$ : It should be chosen based on the desired workspace of the slave, required precision and the maximum force that is desired to be displayed by master device. For instance, a large value of $\lambda$ increases the workspace that can be covered in pose mode, but it compromises the achievable precision.

Moreover, the change in the scaling should occur in a smooth manner. This 
avoids unnecessary inconvenience for the operator due to sudden changes in the force feedback and the control input to the UAV.

Initializing Energy Tanks: Proper initialization of the energy tanks is required in order to accomplish tasks that have associated energetic costs. This can be achieved in various ways. For instance, the primary energy tank can be prefilled with an energy equal to the maximum kinetic energy that one would like the virtual vehicle to acquire at the current mapping scale [69]. The backup energy tank can be prefilled with the additional energy required by the virtual vehicle when teleoperated by the maximum mapping scale [70].

More specifically, by proper choice of the transformation matrix $\Omega$ in (2.5), one can associate the initial state of the primary tank with the maximum velocity that can be acquired by the virtual vehicle. This can be accomplished if the supervisor assigns $\Omega=\bar{Z}^{T} \operatorname{diag}\left(\gamma_{i} \bar{W}_{b v-c}^{b v}(i) / y_{x}(i)\right)$ for $i \in 1,2,3,4$. The entries of $\gamma$ are binary logical inputs that determine the energy flow between the virtual vehicle and the primary energy tank. $\gamma$ is assigned as follows:

$$
\gamma(i)= \begin{cases}0 & (x(i)-l(i))<\mu \text { and } \bar{W}_{b v-c}^{b v}(i) \bar{T}_{b v}^{b v, 0}(i)>0 \\ 1 & \text { otherwise }\end{cases}
$$

where $\mu$ is a very small positive number introduced to avoid numerical singularity. Viscoelastic Coupling and Low-level Controller: As explained in Section 2.5, the low-level controller can be any type of controller depending upon the task to be accomplished. For practical implementation, the viscoelastic coupling can simply be estimated from the relative position and velocity of the virtual vehicle from the real slave. However, for the virtual vehicle to reliably convey the state of the real slave, the viscoelastic coupling between the two should be strong, see Appendix B. Bumpless Switching Controllers and Low-pass Filters: To avoid nonsmooth changes in the control input of the slave system, the switching controller discussed in Section 2.4.3 is designed to be bumpless. Moreover, low-pass filters are incorporated in the control structure to moderate nonsmooth control inputs and force feedbacks that may occur due to switching, significant variable time delays, and packet losses. However, the bandwidth of the filter should be tuned in a way that it adds no significant delay to the controlled system.

Limiting Force Feedback: Often the maximum force displaying capability of haptic interfaces is higher than the force with which the operator can work comfortably. So, to maximize the overall performance while using the haptic feedback, the maximum force that can be displayed to the operator should be limited based on the operator's capability and comfort.

Remark 2.3 The proposed tele-control structure ensures somewhat arbitrary modification of the signals exchanged between the master and slave without compromising the passivity of the high-level teleoperation loop. Hence, the bumpless switching and low-pass filters, and limiting the force feedback that are included to improve the overall performance of the system response, and network-induced imperfections such as significant time-varying delays and packet losses that may be present do not affect the theoretical analysis. 


\subsubsection{Experimental Results}

In this section, representative experimental results obtained by practically realizing the proposed algorithm are presented. All the experiments are carried out in an indoor environment of size $3 \mathrm{~m} \times 3 \mathrm{~m} \times 2.5 \mathrm{~m}$. As a result of the limited workspace, the used mapping scale is $\lambda=4$. Moreover, normal and reset submodes of the pose and rate modes, respectively, are used in the experiments.

\subsubsection{Switching}

In this experiment, the applicability of the switching-based mapping and control algorithm has been verified. Fig. 2.14 shows the obtained results while haptically tele-controlling the UAV in the presence of significant time delays. Specifically, Figs. 2.14a and 2.14b show that the limited stroke of the haptic interface is mapped to either the position or the velocity of the virtual vehicle, based on the state of the switching signal chosen by the operator (the bottom plots in both figures). As can be clearly seen, the virtual vehicle is able to dynamically map the command of the operator and the state of the real UAV because of the presence of a local virtual slave controller and a strong viscoelastic coupling. Fig. $2.14 \mathrm{c}$ shows the haptic feedback displayed to the operator during the course of the experiment. Overall, the result shows the applicability of the proposed algorithm.

\subsubsection{Task-based evaluation}

Several experiments have been conducted to perform a task-based evaluation of the proposed control algorithm. Different subjects, both male and female, have been involved in carrying out the experiments. The subjects have been informed of the task a priori and have carried trial experiments to get familiar with the setup. Each task contains both trajectory tracking and hovering at two distinct positions (see Fig. 2.15). Subjects are informed to track the trajectory and hover for $5 \mathrm{~s}$ at each hovering point. For fair comparison of the achieved performance improvement, every subject has performed four experiments, rate-based and switching-based with and without the presence of time delays.

Table 2.2 shows the metric, based upon which the methods are evaluated, and the results obtained. During the evaluation, experiments where high deviations have been registered are discarded. In both rate and switching based controls, the tracking part is performed in velocity control mode. Therefore, ideally, similar results can be obtained. As a result, the comparison mainly focuses on the accuracy of the hovering position.

The results show that higher accuracy is obtained while using the switchingbased method than the rate-based alone when $\tau=0$. When $\tau=1 \mathrm{~s}$, the error in hovering position accuracy in both methods increases, but yet, the performance of the switching-based is far better. Note that the deterioration in accuracy while using rate mode alone is significant in the presence of time delay.

In addition, the experiment also verified the robust stability of the high-level teleoperation loop under the command of different operators. 

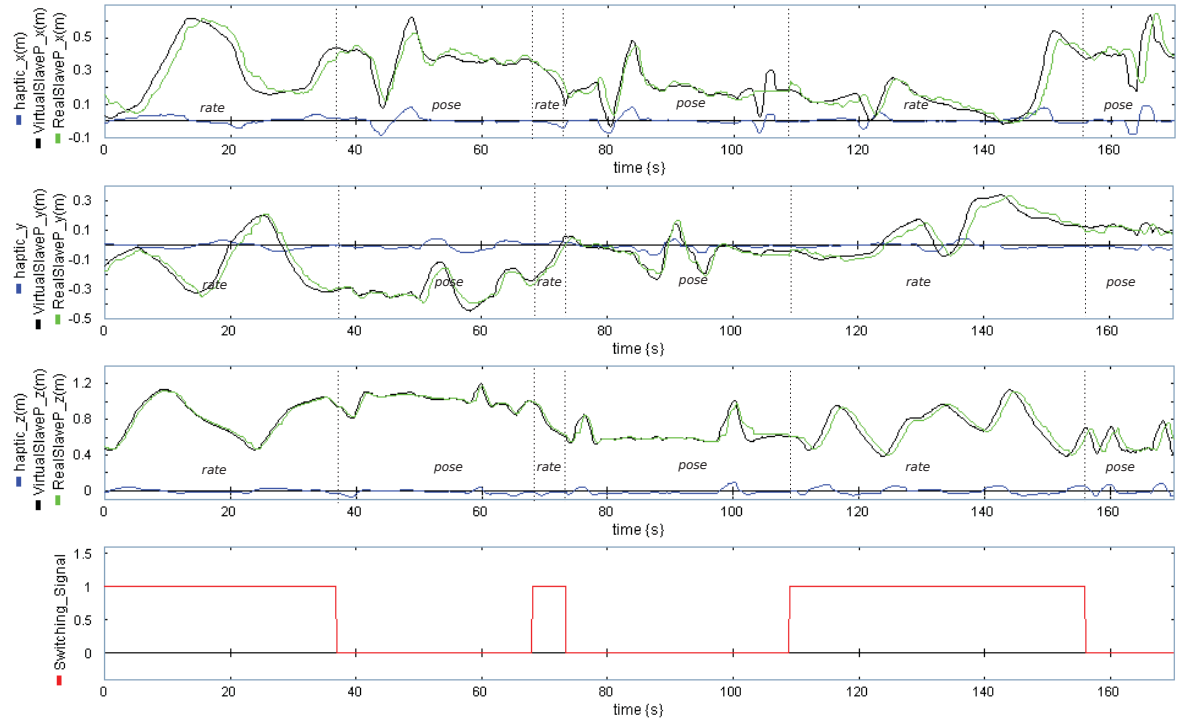

(a) 3D positions of the virtual and real slave.
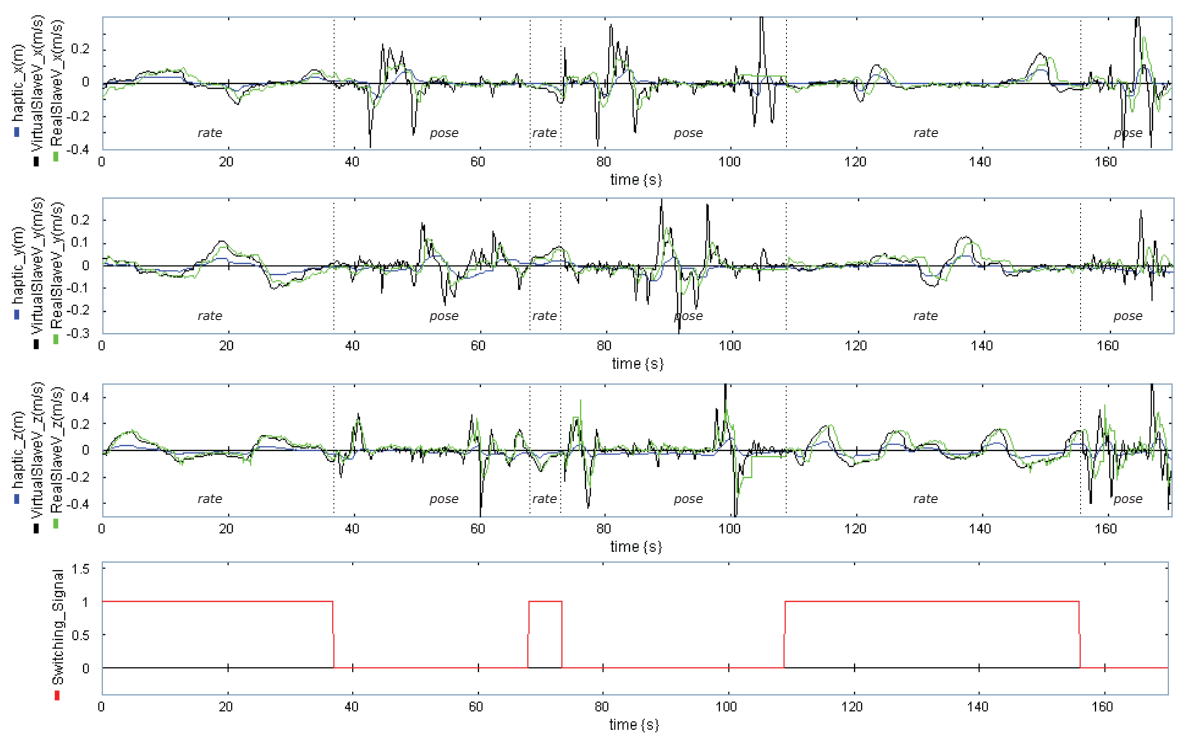

(b) $3 \mathrm{D}$ velocities of the virtual and real slave

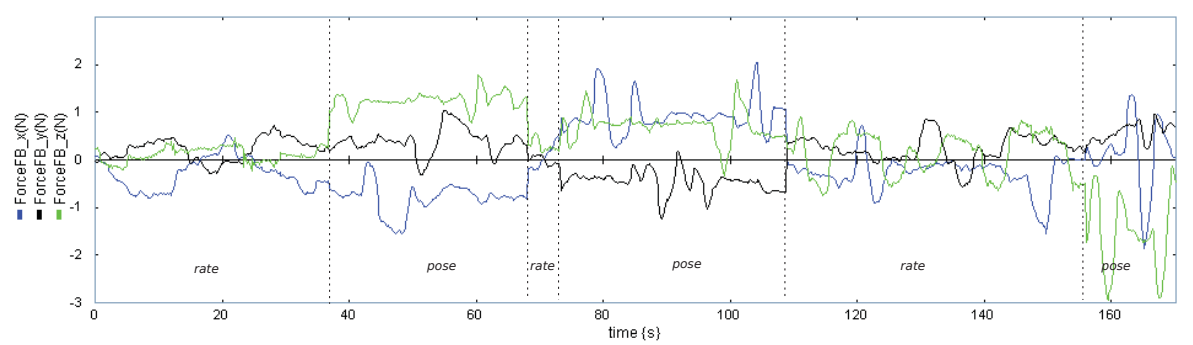

(c) Force Feedbacks.

Figure 2.14: Experimental results. 


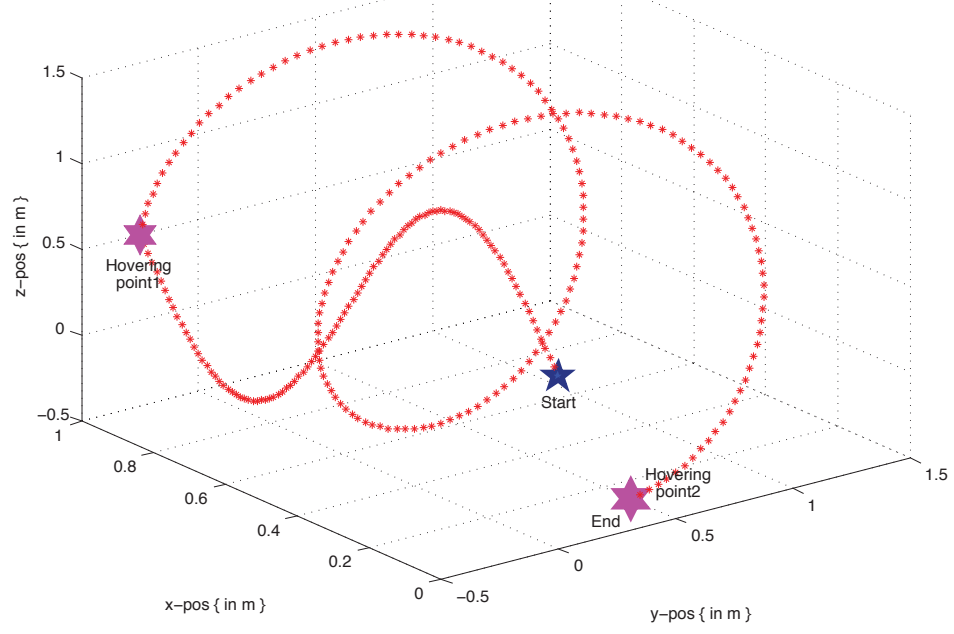

Figure 2.15: The desired trajectory. It contains path following and hovering at the desired hovering points for $5 \mathrm{~s}$.

\begin{tabular}{cccccc}
\hline \hline Mode & \multicolumn{5}{c}{ Evaluation matrices } \\
& \# expt. & err. (ave.) & err. (max) & err. (s.t) & Time \\
\hline \hline $\begin{array}{c}\text { Rate } \\
(\tau=0)\end{array}$ & 36 & 0.0096 & 0.0248 & 0.02 & 96.51 \\
\hline $\begin{array}{c}\text { Switching } \\
(\tau=0)\end{array}$ & 36 & 0.006 & 0.0207 & 0.0111 & 102.26 \\
\hline $\begin{array}{c}\text { Rate } \\
(\tau=1 \text { sec })\end{array}$ & 36 & 0.0162 & 0.1276 & 0.0717 & 122.83 \\
\hline $\begin{array}{c}\text { Switching } \\
(\tau=1 \text { sec })\end{array}$ & 36 & 0.0072 & 0.0368 & 0.0172 & 111.72 \\
\hline
\end{tabular}

Table 2.2: Results of the task-based evaluation experiments. All the measuring metrics are given in SI units.

\subsection{Conclusion}

This chapter described a generic haptic teleoperation control architecture for aerial robots. The presented control architecture comprehensively addresses classical challenges, such as stability and additional peculiar challenges such as workspace limitation of the master and underactuation of the slave.

The proposed variable impedance master controller and the virtual slave system have made the control architecture applicable in a wide range of tasks and operating conditions. Moreover, the proposed mapping strategy that bridges the gap between the conflicting controller design requirements, often implemented to address the inherent workspace incompatibility between the master and the slave and the achievable precision during a task, has greatly increased the versatility of the control architecture. Overall, the fact that the high-level teleoperation loop remains passive in all conditions has made it usable in a plug-and-play fashion with different types of aerial vehicles, along with their low-level controllers and haptic 
interfaces. The passiveness of the high-level is attributed to the energy tanks that the master and the slave are endowed with, and their coupled dynamics that enforces passivity. The clear distinction of the energy circuits of the master and the slave from the modulating signals exchanged between them to modify their respective dynamic behavior has been advantageous. It primarily eliminates additional traffic in the communication channel, avoids unavailability of energy trapped in the channel, and makes the proposed algorithm simple and elegant.

Addressing the suggested practical consideration have greatly contributed to the applicability of the control architecture. Simulations and experimental results verified the effectiveness of the proposed algorithm, even in the presence of significant time delays. Particularly, the conducted task-based evaluation has shown that better precision can be achieved with the proposed algorithm. Although the main objective of the control architecture, from task viewpoint, is teleoperation of aerial vehicles, it can be applied without or with simple modification to other fields of robotics. 



\section{CHAPTER 3}

\section{Transparency in Haptic Teleoperation of AERIAL RoBots}

In spite of the rapidly growing field of haptic teleoperation of aerial robots, and development of stable tele-control strategies, very little attention has been given to improving transparency of the user interface. A key contribution of this chapter is to extend the classical manipulator definition of transparency to mobile robots in noninteractive tasks. In this context, measures of transparency deviation with and without the presence of time-delays are provided. To minimize the transparency deviation that stems from technological limitations, a passive control architecture that incorporates transparency enhancing methodologies is proposed. The control architecture is applicable to both impedance and admittance tele-control frameworks. The effectiveness of the proposed control architecture, with respect to the achievable transparency, is validated via extensive simulations and experiments that also include intercontinental tasks.

This chapter is an adapted composition of (Mersha et al. 2013); Mersha, A. Y., Xiaolei, H., Mahony, R., Stramigioli, S., Corke, P., and Carloni, R., "Intercontinental haptic teleoperation of a flying vehicle: a step towards real-time applications", In Proceedings of the IEEE International Conference on Intelligent Robots and Systems, 2013, and (Mersha et al. 2014d); Mersha, A. Y., Xiaolei, H., Mahony, R., Stramigioli, S., and Carloni, R., "Transparency in Haptic Teleoperation of Aerial Robots", IEEE Transactions of Robotics, 2014, Under review. 


\subsection{Introduction}

Ever since the introduction of the first electro-mechanical master-slave system by Goertz in Argonne National Lab in 1954 [27], the field of telerobotics has expanded greatly. The field encompasses telemanipulation of linear and nonlinear systems [93], real and virtual systems [12], [74], single and multidimensional robots [29], kinematically similar and dissimilar master and slave robots [23], [101], at different scales [99] with and without the presence of non-negligible network-induced imperfections, such as time-varying delays and packet losses [12], [4]. Their application ranges from space [106] to medical surgery [112], and includes production, security, virtual environment, and underwater robotics [31].

Various teleoperation control architectures have been designed to work with the aforementioned types of robotic systems and in different operating conditions, such as linear and nonlinear controllers [5], passivity based controllers [30], adaptive controllers [41], model predictive controllers [77].

These controllers are designed with the main objective of facilitating the projection of the operator's actuation and sensing capabilities to a physically and/or intellectually remote environment, while reducing cost, increasing safety, and improving task-performance. While simplified and generic control architectures have been initially proposed, they are constantly adapted to accommodate wide range of applications and to work in various operating conditions. Besides adaptation of existing control schemes, overcoming peculiar challenges that new application domains pose necessitates the development of new control architectures, such as teleoperation of aerial robots, see [111], [36], [54], [69], [94].

Mobile robots, such as ground robots, underwater robots and aerial robots, have transformed and diversified the employment of robot to unstructured and cluttered environment for handling dangerous and complex tasks, such as waste disposal, and radioactive material handling. Their deployment for complex tasks, such as power plant inspection by contact [59], in teleoperation setting, however, have posed peculiar challenges, such as workspace incompatibility between the master and the slave robots [71]. The research works in haptic teleoperation of aerial robots have, so far, focused on addressing stability and additional unique challenges attributed to aerial robots, [111], [22], [53].

Although stability of a teleoperator is essential for any teleoperation application, any degree of task-performance cannot be inferred from it. Transparency, on the other hand, is often used as a measure of performance [51]. Transparency in haptic teleoperation of mobile robots, and more specifically of aerial robots, still does not have a formal definition.

The main contribution of this study is a complete treatment of transparency as applied to haptic teleoperaion of aerial robots in both free-flight and interaction. In this context, a vehicle transparency and an environment transparency are defined for noninteractive and interactive tasks of aerial robots, respectively. A mathematical tool to measure the deviation of transparency due to the employment of a specific control architecture and/or technological limitations (such as time-varying delays and packet losses) is also provided. 
To alleviate the transparency degradation due to network-induced imperfections, transparency enhancing methodologies are incorporated in the proposed control architecture. These methods include remote impedance reflection and dynamic kinesthetic boundary, which result in an improved transparency. The transparency analysis explicitly includes tele-control architectures with the commonly used impedance and admittance causalities. Moreover, comparative evaluations of both control frameworks, with respect to their merits and practical implementation challenges, are also provided. The different parts of the proposed control scheme are presented by iconic diagrams, bond graphs, and network representations for readers convenience.

The applicability and effectiveness of the proposed control architecture is verified through extensive simulations and experiments. The experimental results also include the longest intercontinental haptic teleoperation of aerial robots. It involves navigation of an aerial robot at the Australian National University, Australia, by a master station located at the University of Twente, The Netherlands. Practical considerations that are necessary for effective utilization of the proposed theory, and system integration of hardware components are also presented.

The chapter is organized as follows. Section 3.2 briefly presents the state of the art in transparency and its limitations in the field of teleoperation of mobile robots. Section 3.3 redefines transparency for haptic teleoperation of aerial robots. The general control design approach, and specific transparency enhancing methodologies in internet-based applications are presented in Section 3.4. Practical considerations, necessary for proper utilization of the proposed controller, are given in Section 3.5. The effectiveness of the proposed control architecture are demonstrated by simulations and experimental tests given in Sections 3.6 and 3.7, respectively. Discussions are presented in Section 3.8, and finally, conclusions are drawn in Section 3.9.

\subsection{Related Works}

Qualitatively, a teleoperator is said to be transparent if the operator cannot distinguish between direct interaction and interaction through the teleoperator with the environment. The first works in quantifying dynamic transparency were concurrently reported in [118] and [51]. In [118], transparency was evaluated on the basis of three ideal responses, whereas in [51], it was based on the correspondence between the mechanical impedance felt by the operator and the real task impedance. Both works presented their analysis using network topology of a two-port teleoperator, originally used to characterize a generic teleoperator in [29] by drawing network analogy of mechanical systems with electrical networks, as shown in Fig. 3.1 .

A characterization of the functional components comprising the teleoperator [51], based on their frequency dependent gains, has been extensively used to quantitatively analyze the transparency that can be achieved by realizing a specific controller, or to synthesize a controller that achieves a desired level of transparency. 


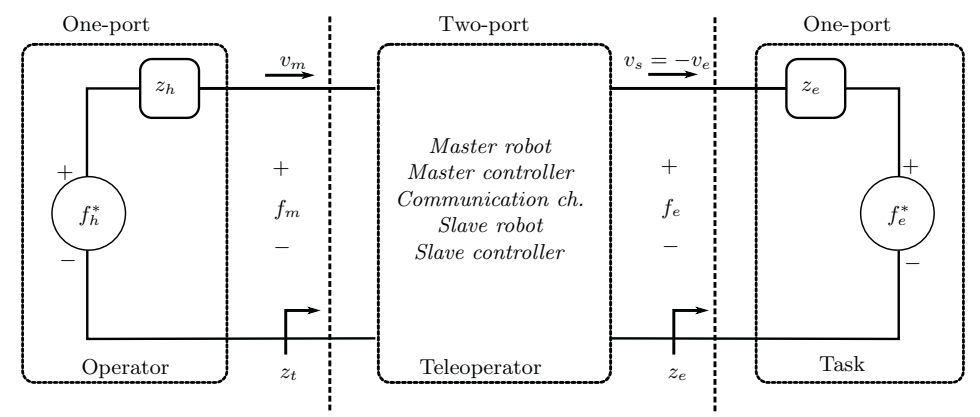

Figure 3.1: Network model of a generic teleoperation control scheme.

Fig. 3.2 shows a generic network representation of a teleoperator, as originally proposed in [51], and extended in [100]. With respect to Figs. 3.1 and 3.2, classical transparency is mathematically described by

$$
\begin{gathered}
z_{t}=z_{e} \\
{\left[\begin{array}{c}
f_{m} \\
-v_{e}
\end{array}\right]=\overbrace{\left[\begin{array}{cc}
0 & 1 \\
-1 & 0
\end{array}\right]}^{\mathbf{H}}\left[\begin{array}{c}
v_{m} \\
f_{e}
\end{array}\right]}
\end{gathered}
$$

where $z_{e}$ is the task impedance and $z_{t}$ is the impedance felt by the operator; and

$$
\mathbf{H}=\left[\begin{array}{ll}
h_{11} & h_{12} \\
h_{21} & h_{22}
\end{array}\right]
$$

in which,

$$
\begin{aligned}
& h_{11}=-\frac{c_{1} c_{4}+\left(z_{m}+c_{m v}\right)\left(z_{s}-c_{s v}\right)}{\left(z_{s}-c_{s v}\right)\left(c_{m f}-1\right)+c_{3} c_{4}} \\
& h_{12}=-\frac{c_{2}\left(z_{s}-c_{s v}\right)+c_{4}\left(c_{s f}-1\right)}{\left(z_{s}-c_{s v}\right)\left(c_{m f}-1\right)+c_{3} c_{4}} \\
& h_{21}=-\frac{c_{1}\left(c_{m f}-1\right)-c_{3}\left(z_{m}+c_{m v}\right)}{\left(z_{s}-c_{s v}\right)\left(c_{m f}-1\right)+c_{3} c_{4}} \\
& h_{22}=-\frac{\left(c_{m f}-1\right)\left(c_{s f}-1\right)-c_{2} c_{3}}{\left(z_{s}-c_{s v}\right)\left(c_{m f}-1\right)+c_{3} c_{4}}
\end{aligned}
$$

where $z_{m}$ and $z_{s}$ represent the master and slave robots impedances, respectively; $c_{m v}, c_{m f}$ and $c_{s v}, c_{s f}$ denote the local force and velocity (position) controllers (subscript $f$ is for force and $v$ is for velocity) of the master and the slave; $c_{i}$, for $i=1,2,3,4$, denotes the force and velocity controllers, whose set-points are sent from the other side across the communication channel.

It is clear from (3.1b) that the ideal teleoperator is energetically neutral, i.e., it does not store, produce, or dissipate energy. This explains why the ideal teleoperator is considered as a massless, infinitely rigid rod [28]. In [79], it is shown that satisfying the following conditions is necessary and sufficient to achieve a global 


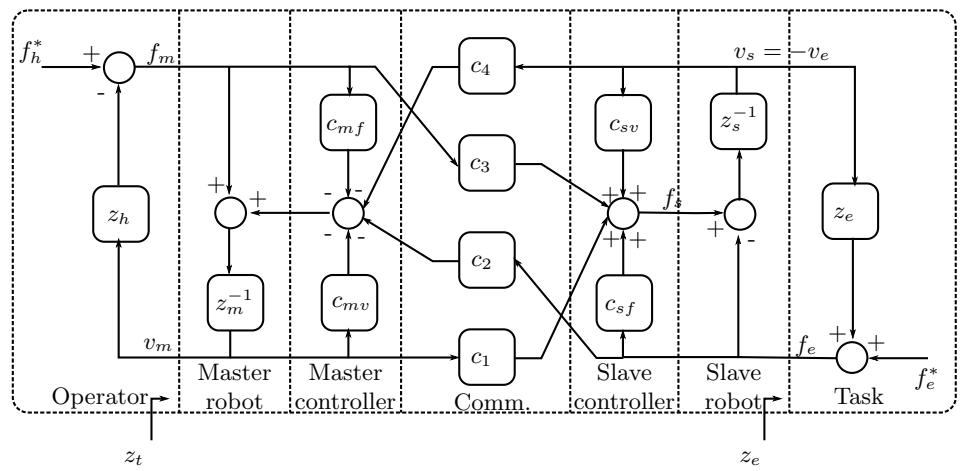

Figure 3.2: The functional block-wise expanded network model of the generic teleoperation scheme shown in Fig. 3.1.

transparency: ${ }^{1}$

$$
\begin{aligned}
& \left(c_{m f}-1\right)\left(z_{s}-c_{s v}\right)+c_{3} c_{4} \neq 0 \\
& c_{1}=z_{s}-c_{s v} \\
& c_{2}=1-c_{m f} \\
& c_{3}=1-c_{s f} \\
& c_{4}=-\left(z_{m}+c_{m v}\right)
\end{aligned}
$$

Various researchers demonstrated that transparency, as given in (3.1), can be achieved by a combination of controllers that satisfy (3.3), using $2-4$ channels, [51], [100], [45].

The transparency conditions given in (3.1b) are derived based on the following implicit assumptions:

- All the parts constituting the teleoperator are linear,

- The master and slave robots have similar kinematics,

- The slave robot is already interacting with the environment.

Furthermore, and more importantly, the above formulation does not account for deterioration of transparency due to mismatch between states of the master and the slave, which are not input or output. For example, correspondence of the force and velocity pairs as in (3.1b), even disregarding network-induced imperfections, does not imply position correspondence. ${ }^{2}$

Even though the transparency conditions in (3.3) have been extensively used to evaluate teleoperation controllers, their application in emerging fields, such as mobile robots, has not been fully investigated and demonstrated. Due to peculiar challenges involving teleoperation of mobile robots, which violates one or more of the underlying assumptions, based on which the transparency conditions are derived, direct adaptation or formulating equivalent notion is necessary.

\footnotetext{
${ }^{1}$ This ubiquitous formulation has been presented in various literature, among others, [50], [51], [99], in various forms.

${ }^{2}$ In some teleoperation algorithms, in which the position information is used to generate the control law, the deviation in position can be implicitly embedded in the exchanged variables.
} 


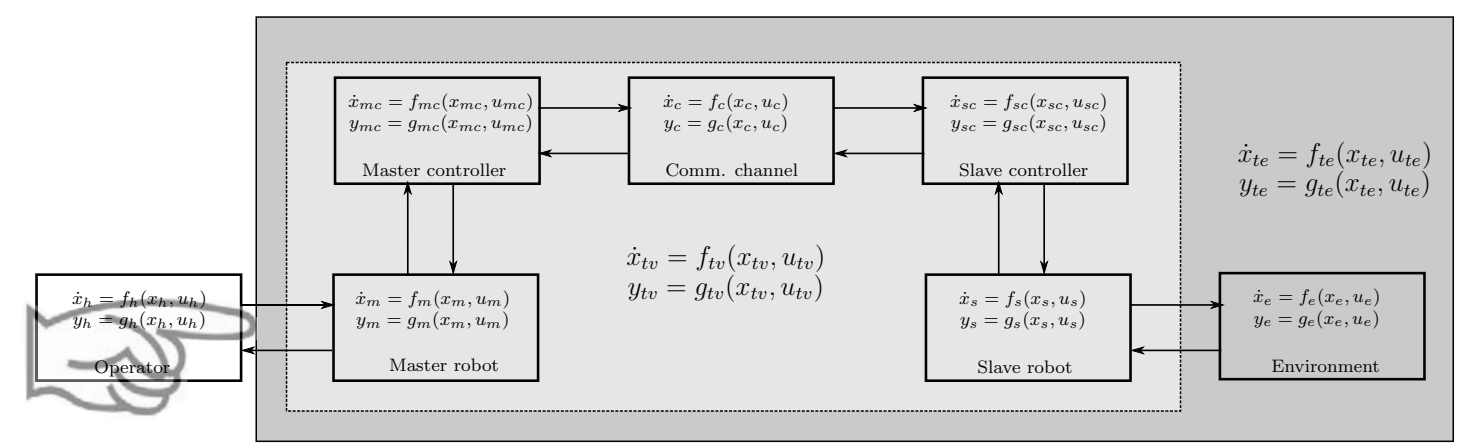

Figure 3.3: A functional block representation of a generic teleoperation scheme, in which each functional block is described in a state space form. With the exception of the operator and the environment blocks, the rest are two-port, and their respective input and output vectors are composition of the inputs and outputs from both ports. The light and dark gray shaded regions represent the composition of the dynamics of each block felt by the operator during free-motion and interaction, respectively.

The only time-domain based transparency definition has been proposed in [103]. Inspired by the framework for interactive robot control proposed in [110], a transparency analysis for a port-Hamiltonian based teleoperation, in which the communication channel is characterized by scattering variables, is proposed. Although this time-domain definition is more general than its most commonly used frequencydomain counterpart, its applicability is only demonstrated in simulation for 1D teleoperation system, whose interconnection between the master and slave system is through power ports. Its application to systems that are represented by a general input-output systems has not been investigated yet.

\subsection{Transparency in Mobile Robots}

In this Section, transparency is redefined in a generic way, such that it also accommodates haptic teleoperation of mobile robots. Since its introduction, different variants of transparency of a telemanipulator have been used as measure of performance. All forms of transparency are defined for tasks involving a manipulation of an environment by using a slave robot. However, in tasks that do not involve interaction with the environment, such as free-flight of aerial robots, the haptic channel designed to achieve maximized transparency in interaction tasks does not provide any information.

In free-flight, for example, the task is to manipulate the slave robot. As a result, the slave robot can not be a part of the teleoperator, rather it becomes part of the task. Hence, to accommodate teleoperation in interactive and noninteractive tasks, transparency is defined here as follows.

\section{Definition 3.1 Transparency}

A teleoperator is said to be transparent if the operator has a timely perception of a direct interaction with the desired behavior of the object of interest.

The key points in the above definition are captured by the following terms: 
- timely perception describes the time-wise match between the behavior perceived by the operator and the actual behavior at the slave side.

- desired behavior refers to the fully/partially controlled or open-loop dynamics of the object of interest. It also embeds the various signal processing and mapping strategies that may be part of the control architecture to enhance the task performance.

- object of interest can be an external environment and/or a slave robot.

The above qualitative definition of transparency is generic and encompasses teleoperations that involve noninteractive maneuver of the slave robot and interactive tasks with the environment. To provide objective tool to measure transparency, an inspiration is drawn from [110] and a time-domain approach is followed. To that end, each one of the seven functional blocks representing a generic teleoperation scheme are represented by a generic state space representation, see Fig. 3.3. Mathematically,

$$
\begin{aligned}
& \dot{x}_{(\bullet)}=f_{(\bullet)}\left(x_{(\bullet)}, u_{(\bullet)}\right) \\
& y_{(\bullet)}=g_{(\bullet)}\left(x_{(\bullet)}, u_{(\bullet)}\right)
\end{aligned}
$$

where $x_{(\bullet)} \in \mathbb{R}^{n}$ is the $n$ - dimensional state of the functional block; $u_{(\bullet)} \in \mathbb{R}^{q}$ and $y_{(\bullet)} \in \mathbb{R}^{p}$ are the input and output of the functional block, with dimensions $q$ and $p$, respectively. $(\bullet) \in\{h, m, m c, c, s c, s, e\}$ denotes the operator, master robot, master controller, communication channel, slave controller, slave robot and environment, respectively. Note that the time dependence of the variables is dropped for brevity.

With respect to Fig. 3.3, it can be seen that the dynamics, which the human operator feels, is a composition of all the dynamics of the remaining blocks. Depending on the occurrence of interaction with the environment, two possible dynamics felt by the human are identified, i.e.,

$$
\begin{gathered}
\left\{\begin{array}{l}
\dot{x}_{t v}=f_{t v}\left(x_{t v}, u_{t v}\right) \\
y_{t v}=g_{t v}\left(x_{t v}, u_{t v}\right)
\end{array}\right. \text { Free-motion } \\
\left\{\begin{array}{l}
\dot{x}_{t e}=f_{t e}\left(x_{t e}, u_{t e}\right) \\
y_{t e}=g_{t e}\left(x_{t e}, u_{t e}\right)
\end{array}\right. \text { Interaction }
\end{gathered}
$$

where $x_{(\bullet)}, u_{(\bullet)}$ and $y_{(\bullet)}$ are the state, the input and the output of the composite blocks. $(\bullet) \in\{t v$ (vehicle teleoperator), te (environment teleoperator) $\}$ denotes the composite of the functional blocks without and with environment, indicated by light and dark gray shades in Fig. 3.3, respectively. Although both the free-motion and interaction dynamics in (3.5) can be uniformly described by one equation, such distinction is deliberately made to emphasize on the different objectives of the tasks during free-motion and interaction.

\section{Definition 3.2 Ideal Transparency}

A teleoperator is said to be ideally transparent if

$$
\left\{\begin{array}{l}
x_{h}-\phi_{t v}^{x}\left(x_{t v}\right)=0 \\
u_{h}-\phi_{t v}^{h}\left(y_{t v}\right)=0 \\
u_{t v}-\phi_{h}^{t v}\left(y_{h}\right)=0
\end{array} \quad\right. \text { Vehicle Transparency }
$$




$$
\left\{\begin{array}{l}
x_{h}-\phi_{t e}^{x}\left(x_{t v}\right)=0 \\
u_{h}-\phi_{t e}^{h}\left(y_{t e}\right)=0 \\
u_{t e}-\phi_{h}^{t e}\left(y_{h}\right)=0
\end{array} \quad\right. \text { Environment Transparency }
$$

where $\phi_{(\bullet)}^{*}$ is a mapping function that establishes the desired correspondence between the states, inputs and outputs of the human operator and the manipulated object of interest, i.e., the slave robot and/or the environment. ${ }^{3}$

The above mathematical definition of ideal transparency does not make any assumptions, confirming its generality. It can be used for any dynamic linear and nonlinear systems, with various mapping strategies, and for teleoperation schemes with admittance and impedance causalities. Note that the classical condition for ideal transparency given in $(3.1 \mathrm{~b})$ is a specific case of $(3.6)$, where the input-output pairs are velocities and forces. ${ }^{4}$

To evaluate the achievable transparency by employing a specific control algorithm, analyze the effect of various components of the teleoperation scheme on the achievable transparency, or even synthesize a controller with a desired level of transparency, it is desirable to have a measure of transparency deviation. In general, however, achieving ideal transparency, as given in Definition 3.2 may not be necessary or desirable to accomplish a task. Hence, not all the states, inputs, and outputs have the same level of importance.

\section{Definition 3.3 Transparency deviation}

The transparency deviation $\Delta$ at time $t$ is given by

$$
\Delta(t)=\left\{\begin{array}{l}
\Delta^{v}(t)=\left\|\begin{array}{c}
\delta_{x}\left(x_{h}-\phi_{t v}^{x}\left(x_{t v}\right)\right) \\
\delta_{u}\left(u_{h}-\phi_{t v}^{h}\left(y_{t v}\right)\right) \\
\delta_{y}\left(u_{t v}-\phi_{h}^{t v}\left(y_{h}\right)\right)
\end{array} \Delta_{\delta_{x}\left(x_{h}-\phi_{t e}^{x}\left(x_{t e}\right)\right)} \Delta^{e}(t)=\right\| \\
\delta_{u}\left(u_{h}-\phi_{t e}^{h}\left(y_{t e}\right)\right) \\
\delta_{y}\left(u_{t e}-\phi_{h}^{t e}\left(y_{h}\right)\right)
\end{array} \|\right.
$$

where $\Delta^{v}$ and $\Delta^{e}$ denote the transparency deviations in free-motion and interaction, respectively; $\|\bullet\|$ denotes the Euclidean norm; $\delta_{(\bullet)}$ is a weighing matrix of appropriate dimension that associates a level of importance. The entries of $\delta_{(\bullet)}$ are between 0 and 1.

\subsubsection{Transparency in the Presence of Time Delay}

In the presence of time delay, transparency, as defined by Definition 3.1 and 3.2, can only be achieved if a perfect model of the human and the manipulated object of interest is embedded in the slave and the master controllers, respectively. As such,

\footnotetext{
${ }^{3}$ The transparency in free-motion is called vehicle transparency because the primary focus of this work is haptic teleoperation of aerial robots.

${ }^{4}$ For the ideal transparency given in $(3.1 \mathrm{~b}), \phi_{t e}^{h}\left(y_{t e}\right)=f_{e}$ and $\phi_{h}^{t e}\left(y_{h}\right)=v_{m}$.
} 


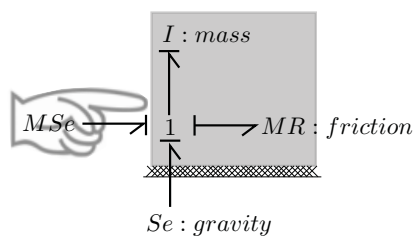

(a) Direct interaction with an aerial robot.

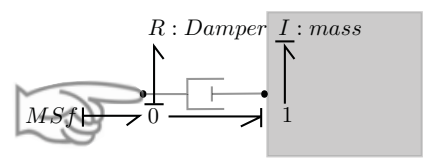

(b) Direct interaction with a controlled aerial robot. The proportional velocity controller is just one example.

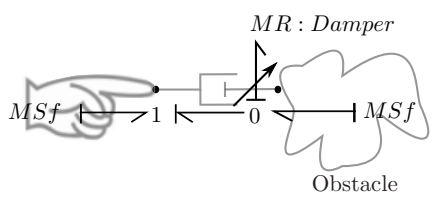

(c) Direct interaction with a environment. Obtacle avoidance type interaction with the environment is just one example.

Figure 3.4: A 1D iconic and bond graph representations of alternatives of direct interactions that can be emulated by the teleoperator during free-flight and interaction of an aerial robot. The aerial robot is represented by its dominant inertial dynamic behavior. Its free-flight and interaction controllers are represented by velocity controllers, with impedance causality.

the transparency degradation due to network-induced imperfections is countered. Nevertheless, modeling the dynamics of the human operator is not easy.

In general, the operator is the source of stimulus, as s/he defines the task. On the other hand, the slave controller is responsible for achieving the desired behavior as commanded by the operator. Hence, time-wise collocated cause and effect perception of the dynamics of the object of interest by the operator is more important for safe and successful task execution. Therefore, in time-delayed teleoperation, it is proposed to evaluate the transparency deviation at the human port, with respect to the ideal behavior.

\section{Definition 3.4 Transparency deviation in the presence of time delay} The transparency deviation $\tilde{\Delta}$ at time $t$ in time-delayed teleoperation is given by

$$
\tilde{\Delta}(t)=\left\{\begin{array}{l}
\tilde{\Delta}^{v}(t)=\left\|\begin{array}{l}
\delta_{x}\left(x_{h}-\phi_{t v}^{x}\left(\tilde{x}_{t v}\right)\right) \\
\delta_{u}\left(u_{h}-\phi_{t v}^{h}\left(\tilde{y}_{t v}\right)\right) \\
\delta_{y}\left(\tilde{u}_{t v}-\phi_{h}^{t v}\left(y_{h}\right)\right) \\
\delta_{x}\left(x_{h}-\phi_{t e}^{x}\left(\tilde{x}_{t e}\right)\right) \\
\delta_{u}\left(u_{h}-\phi_{t e}^{h}\left(\tilde{y}_{t e}\right)\right) \\
\delta_{y}\left(\tilde{u}_{t e}-\phi_{h}^{t e}\left(y_{h}\right)\right)
\end{array}\right\|
\end{array} \|\right.
$$

where $\tilde{\Delta}^{v}$ and $\tilde{\Delta}^{e}$ denote the transparency deviations in the presence of time delay in free-motion and interaction, respectively; the tilde ${ }^{\sim}$ represents the input, state, output variables of the composite block (the shaded areas in Fig. 3.3) at the human port in ideal teleoperation.

It should be noted that it is also possible to evaluate the behavioral deviation at the slave robot side as well, see [103], [110].

\subsubsection{Transparency in Aerial Robot Teleoperation}

In this and subsequent sections, the transparency definition given in the previous section is applied to haptic teleoperation of aerial robots. Establishing a percep- 
tion of direct interaction in haptic teleoperation of aerial robots requires a proper choice of the dynamics to be perceived by the operator. Fig. 3.4 shows some alternatives of the dynamics of the manipulated object, the aerial robot and/or the environment, that the teleoperator attempts to establish direct interaction with. The first alternative attempts to create direct maneuvering of the aerial robots by the operator, where the operator is also responsible to compensate for the action of gravity, friction, and other undesired dynamics. However, such situation quickly exhausts the operator's ability to teleoperate the robot. The second alternative attempts to establish the operator's interaction with a controlled aerial robot, where unwanted forces and higher dynamics are already hidden by the action of the slave controller. The third alternative attempts to establish a direct interaction with the environment.

On the other hand, since the DOFs of the aerial robot that the human operator is interested in haptically tele-controlling are often multiple, different DOFs may be in different flight regimes. For instance, in sliding on a surface while interacting with the environment, or obstacle avoidance in horizontal plane while free-flying in the vertical direction include both free-flight and interaction. Hence, transparency of each DOF of the aerial robot is evaluated independently based on the flight regime it is in. In this work, transparency is measured with respect to the ideal dynamic behaviors shown in Figs. 3.4b and 3.4c, in free-flight and interaction, respectively. Note that achieving ideal vehicle and environment transparencies, even without the presence of network-induce imperfections, requires switching between dynamics of the controlled aerial robot and the environment, based on the flight regime.

\subsection{Tele-control Architecture to Enhance Trans- parency}

In this chapter, a control architecture similar to the previous chapter, which is based on passivity approach using the virtual slave concept, is adopted. Fig. 3.5 shows the iconic and the bond graph representations of the control architecture, with all the necessary parameters and variables used in this chapter labeled. The control architecture shown in Fig. 3.5 is composed of the seven common functional blocks constituting a teleoperation control scheme. The unique features of this architecture include the virtual vehicle and energy based variable coupling between the master and the virtual vehicle, and the virtual vehicle and the real aerial robot. The virtual vehicle essentially serves as a dynamic coupling between the master and the real slave. This coupling is realized by the local virtual slave controller and viscoelastic coupling [69]. Local energy tanks that both the master and the virtual slave are endowed with, ensure the passivity of the tele-control loop. Therefore, the passivity of the controller is not discussed any further, see Section 2.7.

As presented in the subsequent sections, this control architecture is extended to achieve improved transparency in the presence of network-induced imperfections. The applicability of the proposed controller is investigated in both impedance and admittance control frameworks. Classification of a teleoperation scheme into 


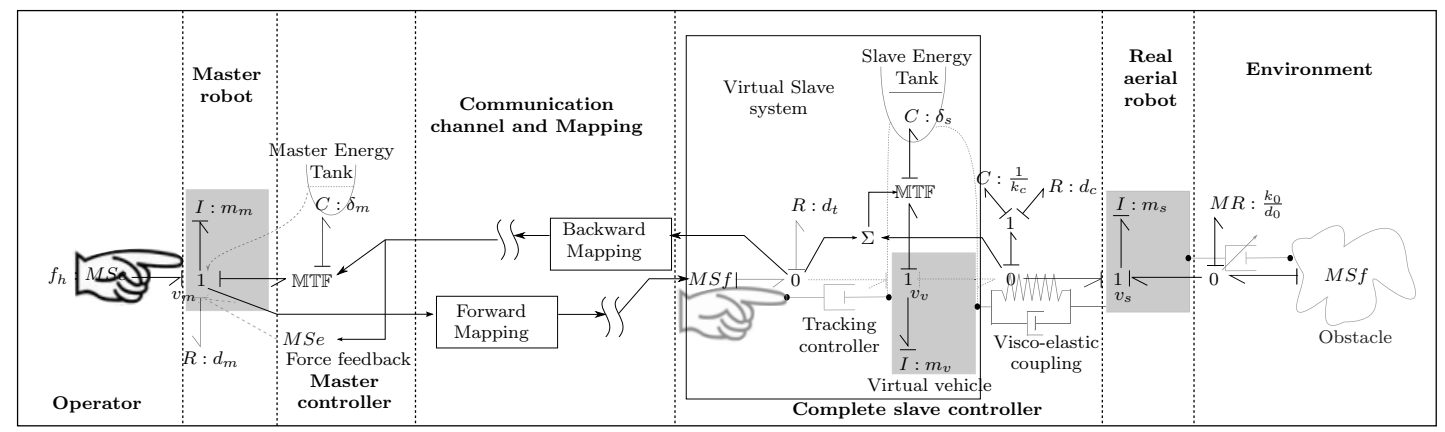

Figure 3.5: The ideal physical model and its equivalent bond graph representation of the complete tele-control scheme. The master and the slave in the teleoperation loop exchange only set-points through the unreliable communication channel. The broken lines connected to the energy tanks show that every control action generated to accomplish the desired task has energetic cost, which can be carried out only when the required amount of energy is available in the local energy tank. The broken half arrow indicates the coupling dynamics realized by the modulated transformer and the energy tank, provided that there is enough energy in the local tank.

impedance and admittance is often based on the causality of the master controller. Further classification based on the causality of the slave controller is also possible. However, in this work, admittance and impedance frameworks refer to the causality of the master controller, and the slave controller's causality in both cases is impedance. In this work, two channel control architecture ${ }^{5}$ similar to the classical P-F and F-P, are used. ${ }^{6}$

The task considered in this work is maneuvering the aerial robot in obstacle strewn environment without collision, and hence, only the translation DOFs of the aerial robot are haptically tele-controlled. Moreover, without loss of generality, a fixed-scale rate-based control strategy is used. With reference to (3.6), the used mapping function is given by

$$
\begin{gathered}
\left\{\begin{array}{c}
v_{v}^{*}(t)=\phi_{t v}^{h}\left(v_{m}(t)\right) \\
f_{m}^{*}(t)=\phi_{h}^{t v}\left(f_{v}((t))\right)
\end{array} \quad\right. \text { Impedance } \\
\left\{\begin{array}{c}
v_{v}^{*}(t)=\phi_{t v}^{h}\left(f_{m}(t)\right) \\
v_{m}^{*}(t)=\phi_{h}^{t v}\left(f_{v}(t)\right)
\end{array}\right.
\end{gathered}
$$

where $\phi_{t v}^{h}$ is an integral function of $v_{m}$ for impedance, i.e., $\phi_{t v}^{h}\left(v_{m}(t)\right):=\lambda_{I} \int v_{m} \cdot d t$, in which $\lambda_{I}$ is a scaling factor; for admittance, $\phi_{t v}^{h}$ is a scalar function of $f_{m}$, i.e., $\phi_{t v}^{h}:=\lambda_{A} \cdot f_{m}$, in which $\lambda_{A}$ is a scaling factor, [35]; $v_{m}$ is the velocity of the master, and $f_{v}$ is the force acting on the object of interest; $v_{v}$ is the velocity of the slave, and $f_{m}$ is the force on the master. The superscript ${ }^{*}$ indicates a possibly scaled reference signal that is sent over the communication channel from the other side of the teleoperation loop.

\footnotetext{
${ }^{5}$ The number of channels indicates the number of position (its derivatives) and force (its derivative) information exchanged across the communication channel.

${ }^{6} \mathrm{P}-\mathrm{F}$ refers to control architectures that send position (its derivatives) from the master to the slave side and send force (its derivatives) from the slave to the master side. In some literature, $\mathrm{P}-\mathrm{F}$ refers to architectures that exchange these signals in reversed order, [45].
} 
To alleviate the transparency degradation in internet-based haptic teleoperation of aerial robots due to time-delays and packet losses, additional extensions of the control architecture in Fig. 3.5 are proposed. The transparency enhancing methodologies include impedance reflection, and dynamic kinesthetic boundary, as described in the following subsections.

\subsubsection{Impedance Reflection}

Although impedance reflection has been used in classical teleoperation schemes [41], [114], its application in the context of aerial robotics is new. The main goal of reflecting the impedance of the remote environment is to bypass the coupling between the master and the slave that suffers from the network-induced imperfections. To achieve reliability in the prediction of the behavior of the remote environment, highly competent local model of the environment is required. The impedance that needs to be reflected in haptic teleoperation of aerial robots is given by both the contributions of the aerial robot and the environment it interacts with. The impedance of the aerial robot can even be identified offline as it does not change during the course of action. To that end, another virtual slave is included at the master side, as shown in Fig. 3.6. But, unlike the virtual slave on the slave side, the mass of this virtual slave is equivalent to the combined mass of the virtual slave on the slave side and the mass of the aerial robot. ${ }^{7}$

On the other hand, the environment that the slave interacts with, such as disturbance due to wind gust, and obstacle, needs to be identified online. This disturbance needs to be reflected at the model of the virtual slave on the master side as well. In the impedance identification process, any environmental disturbance $F_{e}$ that acts on the aerial robot is assumed to be due to the action of a variable damper, which is coupled to the real slave and a certain velocity source. Its dynamics is given by

$$
F_{e}=-d_{e}\left(v_{s}-v_{e}\right)
$$

where $d_{e}$ is the variable damping parameter, and $v_{e}$ is the velocity of the assumed disturbance source.

If $\hat{F}_{e}=-\hat{d}_{e}\left(v_{s}-\hat{v}_{e}\right)$ is the estimation of the environmental force, the estimation error is given by

$$
\begin{aligned}
\tilde{F}_{e} & =\hat{F}_{e}-F_{e} \\
& =\hat{d}_{e}\left(\hat{v}_{e}-v_{s}\right)-d_{e}\left(v_{e}-v_{s}\right) \\
& =\underbrace{\left[\begin{array}{ll}
1 & -v_{s}
\end{array}\right]}_{\Psi^{T}} \underbrace{\left[\begin{array}{c}
\hat{d}_{e} \hat{v}_{e}-d_{e} v_{e} \\
\hat{d}_{e}-d_{e}
\end{array}\right]}_{\tilde{\Theta}}
\end{aligned}
$$

where $\Psi$ is the regressor vector, $\tilde{\Theta}=\hat{\Theta}-\Theta$ is the estimation error.

For estimation of the environmental parameters, the following adaptation law is employed:

$$
\dot{\tilde{\Theta}}=-\gamma \Psi \tilde{F}_{e}
$$

\footnotetext{
${ }^{7}$ The local energy content of this virtual slave should be initialized accordingly, [71].
} 


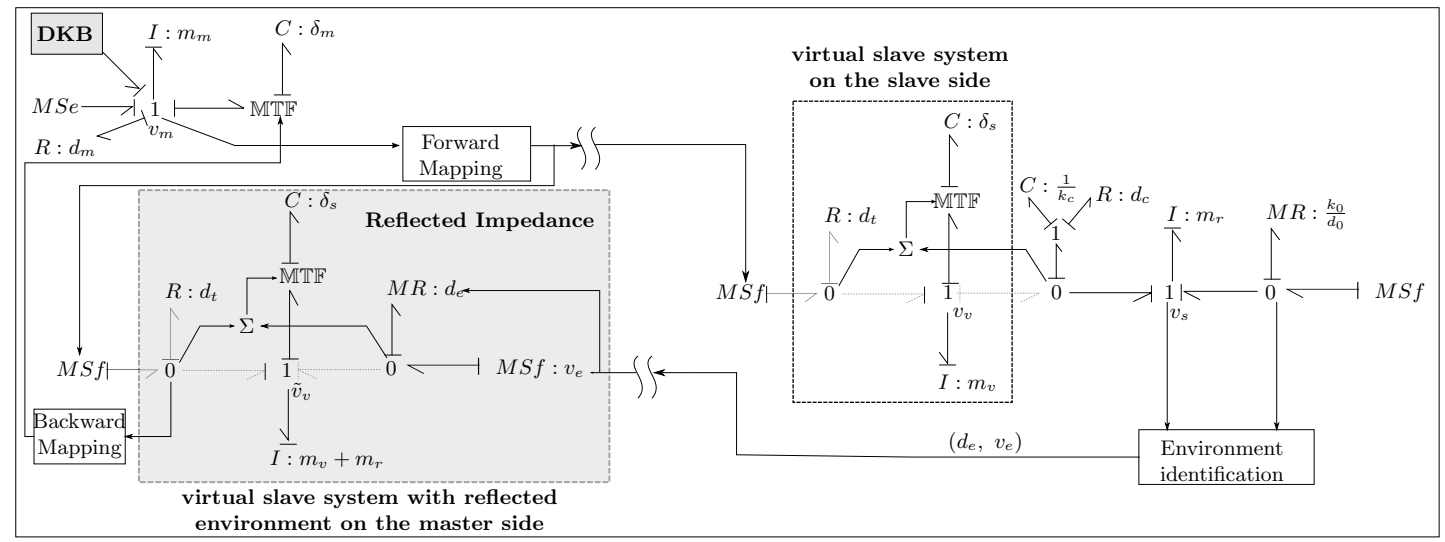

Figure 3.6: Bond graph representation of the proposed control architecture that incorporates the reflected impedance and the DKB at the master side. Note that the parameters of the external environment are identified on the slave side, and hence, the transient phase of the identification process is affected by network-induced imperfections. $\tilde{v}_{v}$ is the velocity of the virtual slave on the master side.

where $\gamma=\operatorname{diag}\left(\gamma_{1}, \gamma_{2}\right)$, with $\gamma_{1}, \gamma_{2}>0$.

Assuming that the environmental parameters are slowly varying with respect to the parameter estimation dynamics, $\dot{\Theta}$ can be safely disregarded, i.e., $\dot{\Theta} \approx 0$. The estimates are, therefore, given by

$$
\left[\begin{array}{ll}
\hat{d}_{e}, & \hat{v}_{e}
\end{array}\right]=\left[\begin{array}{ll}
\hat{\Theta}(2) & \hat{\Theta}(1) \\
\hat{\Theta}(2)
\end{array}\right]
$$

provided that $\Theta(2) \neq 0$. Consider a candidate Lyapunonv function $V:=\tilde{\Theta}^{T} \gamma^{-1} \tilde{\Theta}$, it is easy to verify that the adaptation law given by (3.12) ensures convergence of the estimates, provided that $\Psi^{T} \Psi$ is positive definite, [17].

Impedance reflection can also be intuitively implemented for the admittance framework. Though the admittance configured haptic device can not directly render the environmental force to the operator, the environmental impedance could be perceived indirectly as part of dynamic forces through the change of velocity tracking performance of the virtual slave on the master side due to the environmental force.

\subsubsection{Dynamic Kinesthetic Boundary for Perceiving the Re- mote Environment}

Since the admittance configured haptic interface can not directly render the environmental forces, a Dynamic Kinesthetic Boundary approach (DKB) [34] is proposed to provide the operator with kinesthetic cues (both force and motion) for perceiving remote environment in the workspace of an admittance configured haptic interface. The DKB projects the remote environment to the workspace of the haptic device as a virtual boundary, and modulates the reference to the slave robot in accordance with the virtual boundary for avoidance of possible collisions with obstacles. By exploiting unique kinesthetic cue that is different from the classical 
bilateral force feedback approach, DKB offers clear separation of different haptic sensory channels that enables both vehicle and environmental transparencies at the same time (force cues for vehicle transparency and kinesthetic cues for environmental transparency).

The DKB has not been implemented on impedance configured haptic interface before, but similar approach can be followed. The DKB is constructed with the God-Object on virtual boundary that is derived by the sensor measurement. The position $\beta(t)$ of God-Object can be given by

$$
\beta(t)=\min \left\{\lambda_{I} \frac{\varrho(\theta, \phi, t)-d_{\mathrm{sf}}}{d_{\mathrm{th}}} v_{\max }, \xi_{\max }\right\}
$$

where $\varrho(\theta, \phi, t)$ is the sensor measurement of the obstacle in the bearing of $(\theta, \phi)$ in body fixed frame at time $t, \xi_{\max }$ denotes the maximum mechanical displacement of the impedance haptic interface, $\left(d_{\mathrm{sf}}, d_{\mathrm{th}}\right)$ are the safety distance and the threshold distance to encode thresholds that limit how close the aerial robot will approach obstacles; $v_{\max }$ is the maximum velocity of the slave, i.e., $v_{\max }=\lambda_{I} \xi_{\max }$.

This dynamic boundary is rendered on impedance haptic interface through a contact dynamics. It is proposed to use the Hunt-Crossely contact model [38], i.e.,

$$
f_{\text {env }}^{0}= \begin{cases}k_{d k b} \cdot\left(\Delta^{c}\right)^{n}+d_{d k b} \cdot\left(\Delta^{c}\right)^{n} \cdot v_{m} & \text { for } \Delta^{c}<0 \\ 0 & \text { otherwise }\end{cases}
$$

where $\Delta^{c}=\beta(t)-x_{m}, k_{d k b}$ and $d_{d k b}$ are stiffness and damping parameters of the contact dynamics; $n$ is a constant, which is often between 1 and $2 ; x_{m}$ and $v_{m}$ are position and velocities of the haptic interface.

When the tip of the haptic interface has no contact with the DKB, the operator does not feel the DKB and the haptic interface operates under normal working mode. Otherwise, the operator perceives the DKB, and the haptic interface's workspace becomes bounded by the DKB. Considering the system in (3.9a), the bounds of DKB also bound the maximum velocity reference to the slave robot, and thus obstacle avoidance is achieved in the sense of the DKB converging to the original of the haptic workspace as the slave gets closer to the obstacle.

\subsection{Practical Considerations}

For effective utilization of the proposed control architecture, the following practical considerations, besides those given in Chapter 2, should be taken into account.

Aligning Operator and Robot views: The coordinate frames of the aerial robot and the haptic interface, with respect to which their motions are described, should be adjusted to correspond to the image feedback from the on-board camera. As such, the operator feels as if $\mathrm{s} /$ he is operating the aerial robot from on-board.

Multimodal Feedback: In order to distinguish force feedbacks emanating from the obstacle avoidance and free-flight, it is beneficial to use additional feedback, such as sound.

Selection of $d_{\mathrm{sf}}$ : In the absence of time delays, $d_{\mathrm{sf}}$ can be chosen to be a very small 
safety distance between the aerial robot and potential obstacles. This method can even be employed for close aerial inspection of infrastructures without contact. However, in the presence of time delays, $d_{\text {sf }}$ should be selected based on the upper bound of the round-trip time delay present in the communication channel. Alternatively, for unknown time delays, the translational dynamics control of the aerial vehicle can be augmented by obstacle avoidance forces, as in [67].

Selection of $d_{\mathrm{th}}: d_{\mathrm{th}}$ should be chosen based on both the aerial robot's maximum velocity and volume of the empty space in the environment. High maximum velocity of the robot requires a large threshold $d_{\mathrm{th}}$ for deceleration. Similarly, large volume of empty space leads to large $d_{\mathrm{th}}$ for the operator to perceive the obstacles far away. However, $d_{\text {th }}$ could also be small in large empty space case where the flight close to the obstacles is unlikely and/or interaction with environment is not desired.

Scaling Operator Force Input: Unlike the mechanical constraint on the workspace of an impedance haptic interface, the force applied by the operator on an admittance haptic interface is not bounded, and hence, offers better resolution in the sense of large range of values. Nevertheless, a scaling factor is necessary to adjust the operator force input to provide proper resolution and range of desired references.

\subsection{Simulations}

In this section, simulations illustrating the effect of the proposed transparency enhancing methodologies, with and without the presence of time delays, in both impedance and admittance frameworks, are presented. For comparison in impedance framework, the classical P-F and P-F with impedance reflection, denoted as IR-I, tele-control architectures are implemented. For admittance framework, the classical F-P and F-P with impedance reflection, denoted as IR-A, are implemented. In all the control architectures, a rate-based mapping strategy, as given in (3.9) is used. The transparency deviations are evaluated according to Definitions 3.3 or 3.4, depending upon the presence of time delays.

In the simulations, a 1D dynamic model of the complete architecture is used. The control parameters are tuned according to the guideline provided in Appendix B.

\subsubsection{Impedance Framework}

In the first two simulations, a two channel P-F tele-control architecture with and without impedance reflection (IR-I) is implemented. Since the viscoelastic coupling between the virtual and the real slaves is strong, the states of both slaves are similar. As a consequence, the velocity of the virtual slave at the slave side virtually represents the velocity of the real slave as well. Thus, the velocity of and the force applied to this virtual slave are used as a substitute ${ }^{8}$ for the real slave in

\footnotetext{
${ }^{8}$ If the mass of the virtual slave and the viscoelastic coupling parameters are tuned as suggested in Appendix B, this system serves as a bilateral velocity and force low-pass filter between the
} 
the transparency analysis. In the third simulation, the applicability of the DKB algorithm in impedance framework for obstacle avoidance is illustrated.

\subsubsection{Without network-induced imperfections}

In this simulation, a desired slave velocity is tracked in the presence of environmental disturbance, but without any network-induced imperfection. Fig. 3.7a shows the operator's velocity command, represented by the scaled master position, which is reasonably tracked by the velocity of the slave in both control architectures, with and without the impedance reflection. For the P-F control architecture, when the environmental disturbance shown in Fig. 3.7b is applied to the real slave, the velocity of the slave deviates from the operator's command. This deviation is reflected in the force feedback displayed to the operator, as shown in Fig. 3.7c. This is similar for the IR-I architecture as well, except that there is a slight delay, with respect to its P-F counterpart, due to the delay induced by the impedance identification adaptation law. As it can be seen from Fig. 3.7b, the environmental force estimate, identified through impedance identification law given in (3.12), is comparable to the actual disturbance. For both architectures, the force feedback is dominated by the inertial force of the slave prior to the application of the disturbance, see Fig. $3.7 \mathrm{c}$.

The transparency deviations of both control architectures are evaluated according to Definition 3.3, and the results are shown in Fig. 3.7d. The transparency deviations of both architectures, prior to the application of the disturbance, are similar. These deviations stem from the nonzero inertial and damping forces of the master, which are included in the model to represent part of the master dynamics that is not completely compensated. In general, without the presence of network-induced imperfection the P-F achieves better transparency than the IR-I architecture. This is due to the fact that the delay induced by the adaptation law results in additional deviation between the dynamics felt by the operator and the actual controlled dynamics of the slave.

\subsubsection{With time delay}

In this simulation, the operator attempts to command a similar motion profile for the slave as in the previous simulation, with the presence of a constant time delay of $0.75 \mathrm{~s}$ in both the forward and the backward communication channels. Compared to the results obtained from the previous simulation, the most notable differences are the delayed velocity of the virtual slave in the slave side, and the force feedback displayed to the operator in the P-F architecture, see Figs. 3.8a and 3.8b. On the other hand, in the IR-I architecture, the velocity of the virtual slave on the master side and the force feedback are similar as in the previous simulation, with the exception of the transient during the impedance identification process, see Figs. 3.8a-3.8c.

master and the slave. 


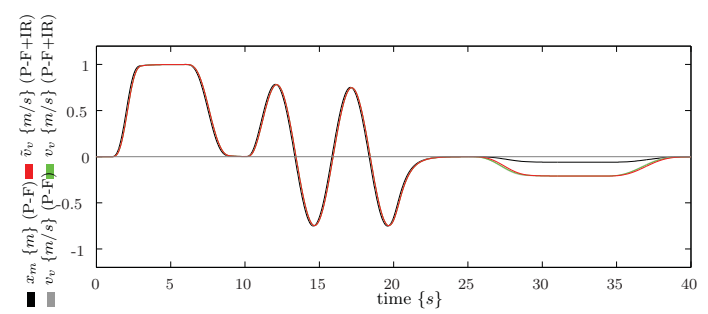

(a) The scaled master position and velocities of the slaves in P-F and IR-I control architectures.

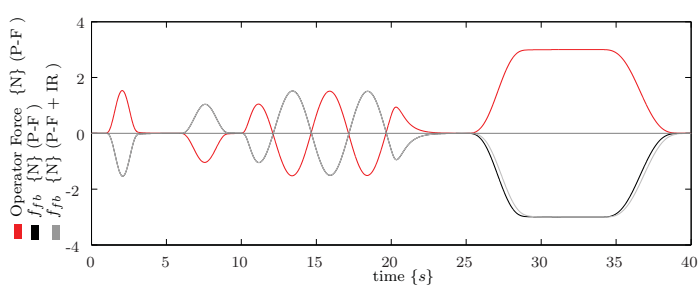

(c) The force feedback displayed to the operator and the force applied by the operator.

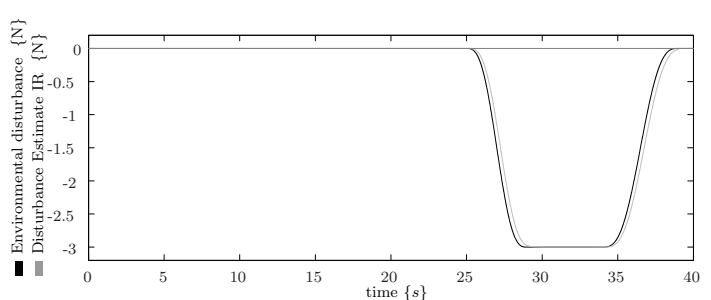

(b) The actual environmental disturbance and its estimate obtained from the identified impedance.

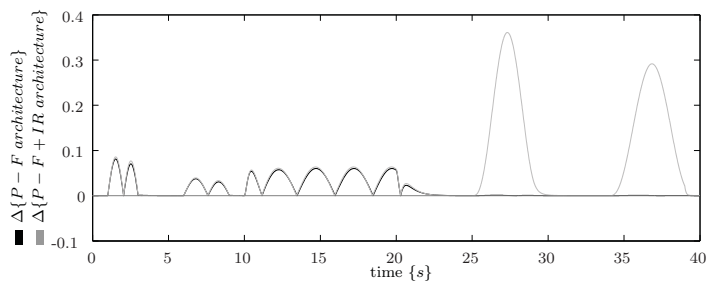

(d) Transparency deviations in P-F and IR-I architectures.

Figure 3.7: Results obtained from the free-flight test in the presence of environmental disturbance without the presence of network-induced imperfections.

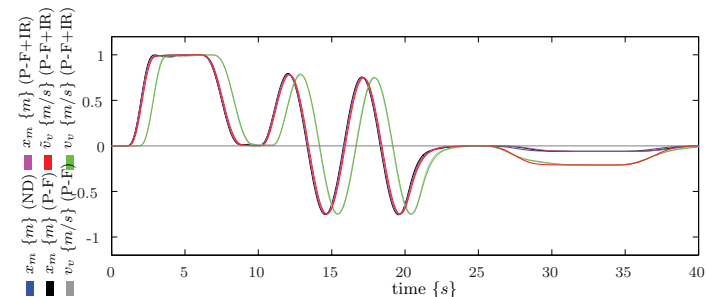

(a) The scaled master position and velocities of the slaves in P-F and IR-I control architectures. $\tilde{v}_{v}$ is the veolity of the virtual slave on the master side, see Fig. 3.6.

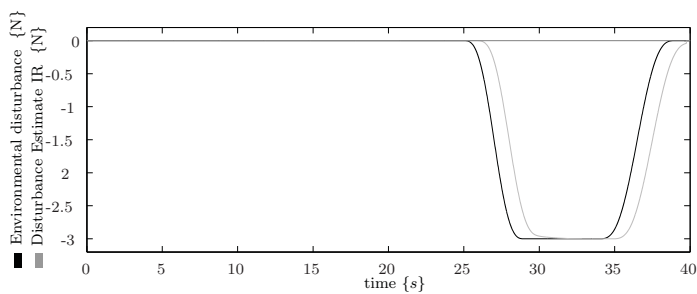

(c) The actual environmental disturbance and its estimate obtained from the identified impedance.

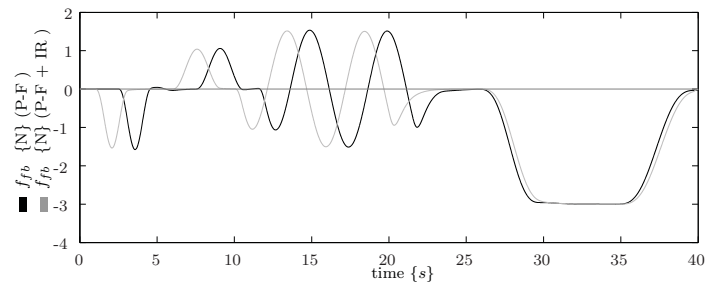

(b) The force feedback displayed to the operator and the force applied by the operator.

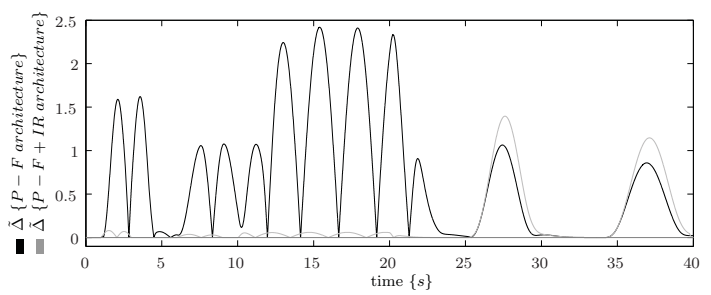

(d) Transparency deviations in P-F and IR-I acrchitectures with the presence of time delays.

Figure 3.8: Results obtained from the free-flight test in the presence of environmental disturbance with the presence of time delays. 
Its clear from Figs. 3.8a and 3.8b that the discrepancy between the operator's command and the force feedback due to the time delay in the P-F architecture creates a distorted perception. The IR-I, on the contrary, does not suffer from this as the operator interacts with the local dynamic model of the remote slave/environment, greatly reducing the impact of time delay on the perception. ${ }^{9}$ The difference in perception is also captured by the transparency deviations shown in Fig. 3.8d, which is evaluated according to Definition 3.4. In general, in the presence of non-negligible time delays, the IR-I architecture outperforms the P-F. Although the transparency of the IR-I architecture decreases during transients of the impedance identifications process (see Figs. 3.8c and 3.8d), it quickly converges to zero once the environmental parameters reach their steady states. Most importantly, the time-wise collocation of the IR provides timely perception of the remote slave/environment, whereas the classical P-F suffers from a lack of it.

\subsubsection{DKB without time delay}

This simulation demonstrates the applicability of DKB algorithm in impedance framework. The task involves free-flight and interaction in the form of obstacle avoidance. In this simulation, the parameters required for the DKB algorithm given in (3.14) are $\xi_{\max }=0.2, v_{\max }=1 \mathrm{~m} / \mathrm{s}$, and the parameters for the boundary constraint given in $(3.15)$ are $k_{\mathrm{dkb}}=1000 \mathrm{~N} / \mathrm{m}$ and $d_{\mathrm{dkb}}=100 \mathrm{Nm} / \mathrm{s}$.

As it can be seen in Figs. 3.9a and 3.9b, the slave moves at the desired velocity according to the operator's command. Once an obstacle is detected, and the slave enters the zone, where obstacle avoidance is activated, the boundary of the master workspace changes dynamically, based on how close the slave is to the perceived obstacle, see Figs. 3.9a-3.9c, and (3.14). Observe from Fig. 3.9b that as long as the operator has no contact with the dynamically changing boundary of the haptic device, there is no environmental force applied on the device.

Fig. 3.9d shows the transparency deviation during the course of this simulation. The deviation is computed according to Definition 3.3 for both vehicle and environment transparencies. In the free-flight, from $0-6.87 \mathrm{~s}$, the deviation is only due to the inertial and damping forces of the haptic device. However, at the start of interaction with the environment, the observed large deviation is due to the fact that the operator is perceiving both the environmental interaction force and the force required to maneuver the controlled slave. Ideal transparency requires switching between the two forces depending upon contact. Hence, this significant environmental transparency deviation is the price paid to avoid switching. Ideal environmental transparency is eventually restored when the slave robot comes to halt.

\footnotetext{
${ }^{9}$ In fact, loosely speaking, for the duration that the remote slave/environment is not varying, the remote slave in IR-I architecture is controlled in feed-forward mode.
} 


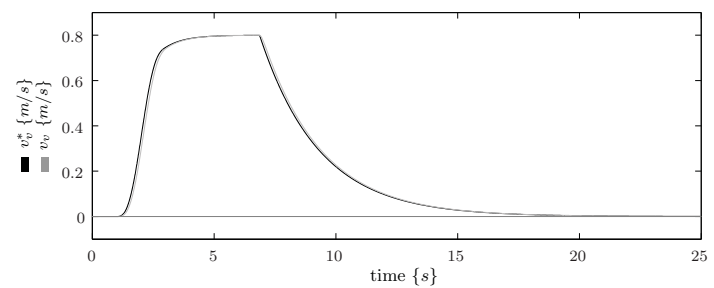

(a) The scaled master position and velocity of the virtual slave.

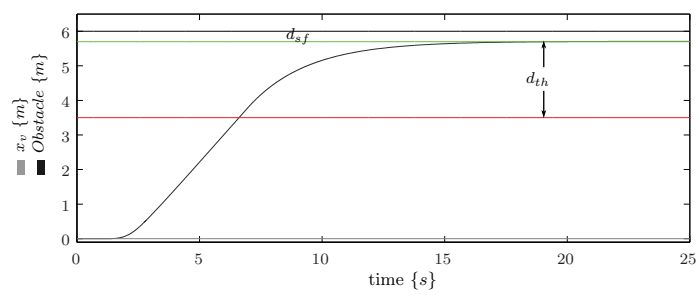

(c) The position of the slave.

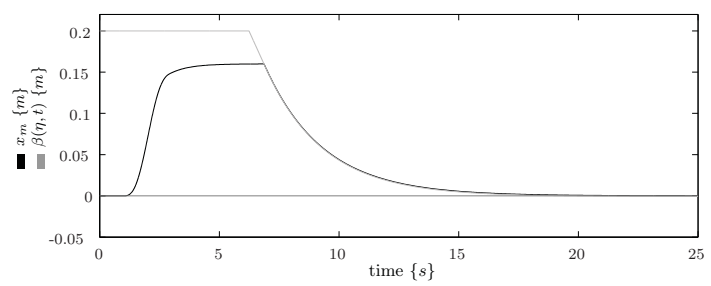

(b) The actual position of the master and its dynamic boundary.

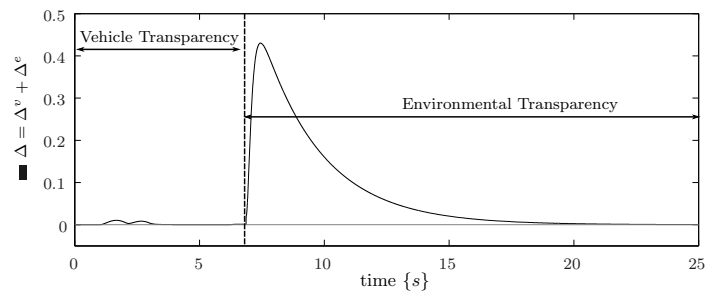

(d) Transparency deviation.

Figure 3.9: Results obtained from a task involving free-flight and obstacle avoidance.

\subsubsection{Admittance Framework}

Two more simulations have been run using two admittance tele-control architectures, two channel F-P, and two channel IR-A. The desired slave motion profile is similar to the first two simulations presented in the previous section. Since the obtained results follow the same trend, as far as the transparency deviation is concerned, only qualitative presentation is provided, while noting their differences.

When there is no network-induced imperfection in the tele-control loop, both the F-P and IR-A show similar transparency deviation. This very small deviation from ideal transparency is mainly due to the delay induced in the filter, which is included to represent the filtration of high frequency operator force input. When an environmental disturbance is applied, the transparency deviation of the IRA becomes larger than the F-P during the impedance identification process. In the presence of non-negligible time delays, however, a far better transparency is achieved using the IR-A architecture than its F-P counterpart. This observation is similar to the observation made when employing similar impedance tele-control frameworks.

A notable difference between the impedance and admittance frameworks is how the environmental disturbance is perceived by the operator. In impedance framework, it is perceived as environmental impedance, as in Figs. 3.7c and 3.8b. However, in admittance framework, it is displayed to the operator as added inertia, requiring more operator force input than actually required to move the slave alone at the same velocity. 


\subsection{Experiments}

In this section, the dynamics of the real slave, its controller and the experimental setup used to realize the longest intercontinental haptic teleoperation of an aerial robot are described. Extensive experimental results using both the impedance and admittance frameworks are also provided.

\subsubsection{The Real Slave}

Both the real aerial robot and its controller are part of the real slave system. In this work, a Mikrokopter quadrotor is used as the aerial robot. The linear translational dynamics of the quadrotor are approximated based on the assumption of quasi-stationary flight, and disregarding aerodynamic induced lift and rotor flapping effects. This leads to the approximation that the thrust $T=m_{s} g$ exactly compensates for the gravitational force.

The $x$ - and $y$-axes translational dynamics are identified and approximated by a second order transfer function from a stick position (pitch and roll for $x$ and $y$ axes, respectively) input to a velocity output, i.e.,

$$
H(s)_{x \mid y}=\frac{a_{(x, y)} \omega_{(x, y)}^{2}}{s^{2}+2 \zeta_{(x, y)} \omega_{(x, y)} s+\omega_{(x, y)}^{2}}
$$

where $a_{()}$is a gain; $\omega_{()}$and $\zeta_{()}$are the resonance frequency and the relative damping ratio of the quadrotor translational dynamics along the $x$ - and $y$-axes.

Similarly, the transfer functions describing the translational dynamics along the $z$-axis and the yaw from stick position input to position output are given by

$$
H(s)_{z, \theta}=\frac{a_{(z, \theta)}}{s^{2}+b_{(z, \theta)} s}
$$

where $a_{()}$and $b_{()}$are gains.

PID controllers on the desired velocity (in $x$ and $y$ ) and position $(z)$ of the real slave, which are defined by the velocities and positions of the virtual slave, are realized based on the above dynamics.

\subsubsection{Experimental Setup}

Fig. 3.10 shows the hardware components and the overall architecture used during the experiment. The main hardware components are briefly described below.

1. Haptic Interface: For impedance framework, the Omega6 device (Force Dimension, Nyon, Switzerland) is used as haptic interface. It has 6DOF position sensing and 3DOF force displaying capabilities. It is endowed with a real-time USB 2.0 controller that enables it to display a high fidelity force up to $12 \mathrm{~N}$ at a rate of $4 \mathrm{KHz}$. Additionally, the device has accurate gravity compensation. 


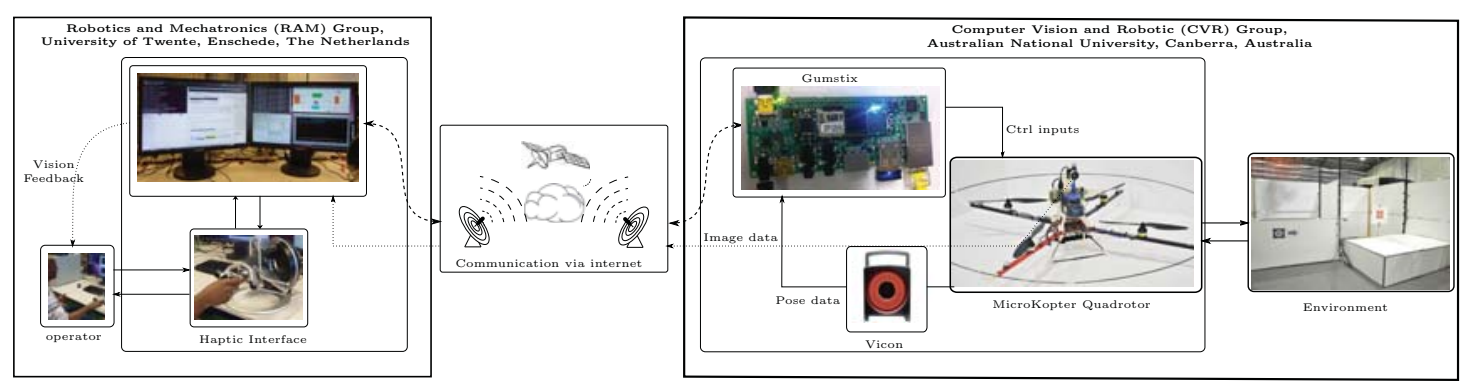

Figure 3.10: The hardware architecture of the experimental set-up used to realize the longest intercontinental haptic teleoperation of an aerial robot.

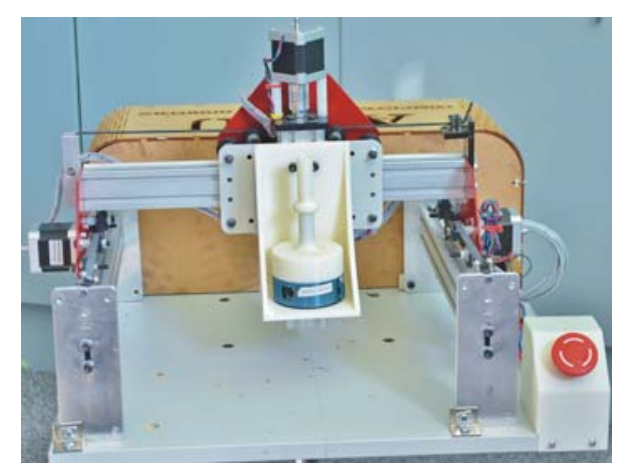

Figure 3.11: The Australian National University's custom built admittance haptic interface.

For admittance framework, a 3DOF admittance haptic interface, custom built at the Australian National University is used, see Fig. 3.11, [34]. It is capable of measuring the force and torque inputs from the operator using a JR3 6-axis force/torque sensor. The firmware of the joystick runs at $1 \mathrm{KHz}$ with a USB interface for receiving control command and feeding back information.

2. Base Station PC: A desktop PC running Ubuntu 10.04 (32 bit) operating system has been used as a ground station. It is used for computing the force rendered by the haptic interface to the operator and as a graphical user interface.

3. Aerial Robot: A Mikrokopter quadrotor equipped with on-board camera, laser scanner and computational resources is used. A gumstix on-board computer, running Linux with ROS, is integrated into the on-board system to receive commands from and transmit data back to the base station.

4. On-board Camera: An analogue on-board camera with 110 deg field of view is used. The operator is provided with image data coming only from this camera. This is to preserve the generality of the hardware framework for various applications as most remote environments, where teleoperation are conducted, are not equipped with off-board cameras. The images from the on-board camera are streamed back to the local base station through a 5.8 
$\mathrm{GHz}$ wireless video transmitter, and then it is displayed to the operator via Skype $^{\text {TM }}$.

5. Laser Scanner: A light weight Hokuyo UBG 04LX-F01 laser scanner (Hokuyo, Osaka, Japan) is mounted on the Mikrocopter, and is used to detect obstacles in the environment for collision avoidance. It works in a range of $4 \mathrm{~m}$, and provides 680 data points per scan over $240 \mathrm{deg}$ field of view at $35 \mathrm{~Hz}$.

6. Flight Area: A closed flight area of $2 \times 4 \times 6 \mathrm{~m}^{3}$, in which obstacles are presented is used as a flying arena. It is equipped with a visual tracking system.

7. Visual Tracking System: Vicon (Vicon, Oxford, UK) is used to provide the absolute position and attitude data with respect to the reference frame of its workspace at $200 \mathrm{~Hz}$. A velocity observer is implemented to estimate the velocity of the slave for the velocity controller.

The software environment is mainly based on the ROS and Matlab Simulink (MathWorks, Natrick, MA, USA).

\subsubsection{Experimental Results}

In this section, results illustrating the practical applicability and effectiveness of the proposed control architecture, both in impedance and admittance frameworks, are provided. The relevant system and control parameters that have been used during the experiments are given in Table. 3.1.

Table 3.1: Parameters \{in SI units\}

\begin{tabular}{|c|c|}
\hline \multicolumn{2}{|c|}{ Quadrotor (real slave) parameters (scalar) } \\
\hline$a_{x}=a_{y}=0.148$ & $a_{z}=0.05$ \\
\hline$\omega_{x}=\omega_{y}=1.187$ & $b_{z}=0.98$ \\
\hline$\zeta_{x}=\zeta_{y}=1.08$ & \\
\hline \multicolumn{2}{|c|}{ PID controller $\left(k_{p}\left(1+\frac{1}{s T_{i}}+\frac{s}{0.02 s+1} T_{d}\right)\right)$ parameters (scalar). } \\
\hline$k_{p(x)}=k_{p(y)}=70$ & $k_{p(z)}=98$ \\
\hline$T_{i(x)}=T_{i(y)}=3.5$ & $T_{i(z)}=11.7$ \\
\hline$T_{d(x)}=T_{d(y)}=0.23$ & $T_{d(z)}=1$ \\
\hline \multicolumn{2}{|c|}{ Virtual slave and master parameters $\left(I_{3 \times 3}\right.$ is a $3 \times 3$ identity matrix.) } \\
\hline$k_{c}=35 I_{3 \times 3}, d_{c}=70 I_{3 \times 3}$ & $d_{t}=17 I_{3 \times 3}$ \\
\hline $\bar{m}_{m}=0.1 I_{3 \times 3}$ & $d_{m}=0.2 I_{3 \times 3}$ \\
\hline
\end{tabular}

Note that all experiments have been conducted in an indoor environment and external disturbances are limited. Only obstacles are considered as external interaction forces. Hence, for the architectures that use the impedance reflection approach, only the offline identified model of the aerial robot is incorporated as the part of the virtual slave on the master side. 

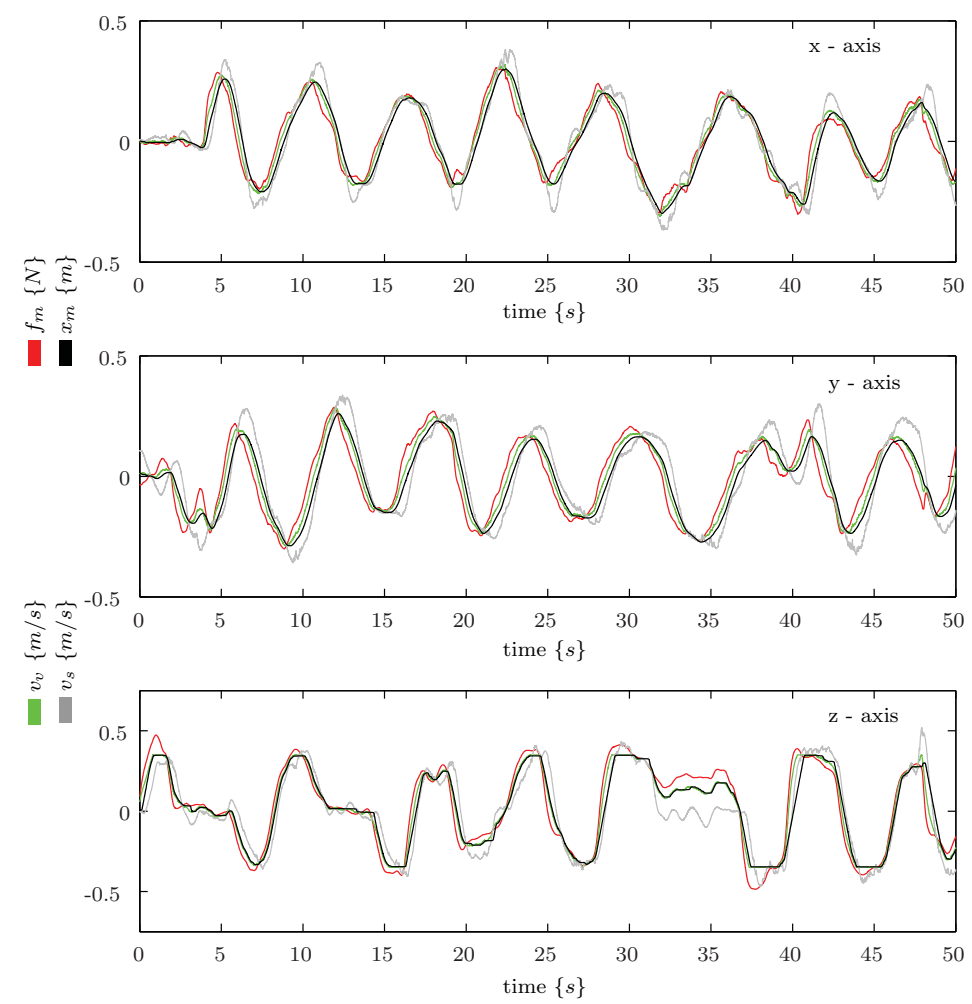

Figure 3.12: The scaled operator force command, the velocities of the virtual vehicle and the aerial robot, and scaled position feedback displayed to the operator.

\subsubsection{Admittance Framework}

In the next two experiments, the proposed controller architecture is tested in admittance framework, with and without time delays, in free-flight and obstacle avoidance tasks.

Free-flight with no time delay This experiment is the first test of the virtual slave concept with an admittance tele-control framework. Fig. 3.12 shows the velocities of the virtual and the real slaves, and the velocity reference derived from the filtered operator force input. The plots in this figure show that the virtual vehicle follows the operator command, and due to the viscoelastic coupling the velocity of the real slave follows this reference as well. The position feedback displayed to the operator during the course of this test is also shown in this figure.

The transparency deviation during this experiment is shown in Fig. 3.13. This deviation is mainly due to the delay induced in the low-pass filter that filters out high frequency operator force input, and the delay in the dynamic response of the admittance haptic interface in displaying the desired position feedback. Moreover, there exists a very small communication delay between the ground PC and the on-board processor of the aerial robot.

Obstacle avoidance using IR-A+DKB, with constant time delay In this experiment, impedance reflection and DKB are used for enhancing both vehicle 


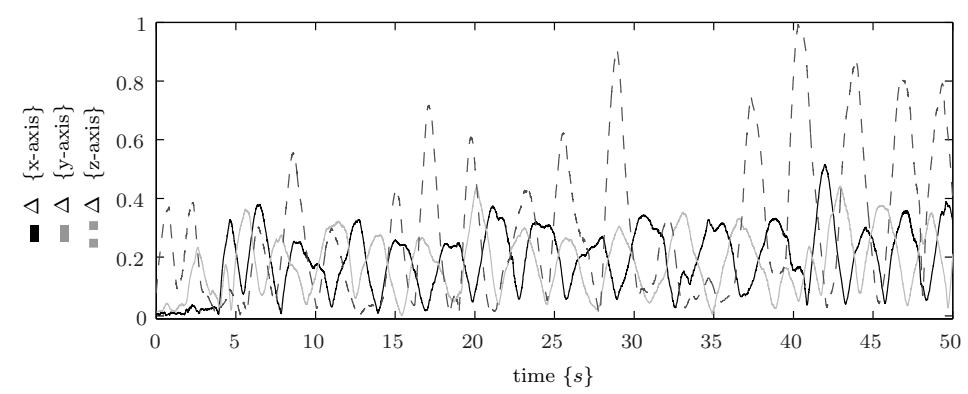

Figure 3.13: Transparency deviation in admittance-based teleoperation using the virtual slave concept without time delay. The deviations are given for the three traslational DOFs.

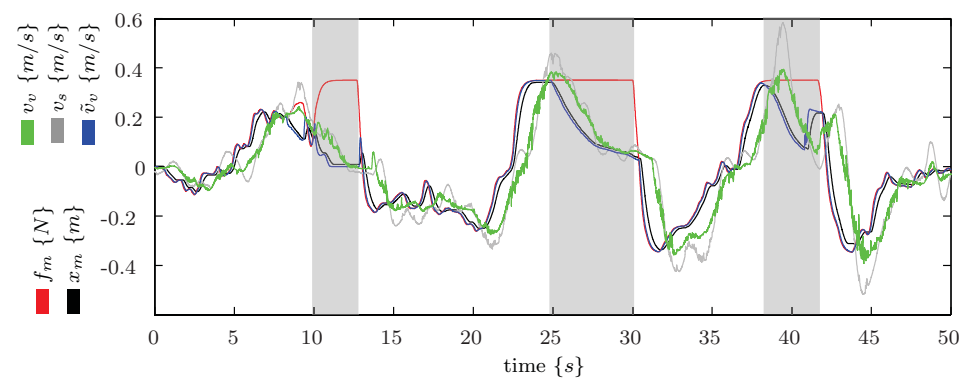

Figure 3.14: Experimental result from an obstacle avoidance test using IR-A+DKB, with the presence of constant time delay. The shaded regions indicate the duration that the DKB has been active.

and environment transparency under admittance configuration of the teleoperation system with $1.5 \mathrm{~s}$ round-trip constant time delay. This constant time delay is deliberately introduced in the control loop. During the course of this experiment, the slave aerial robot has been intentionally commanded by the operator to approach an obstacle in the front, i.e., along the x-axis. The aerial robot has flown normally till it reaches the threshold distance to the obstacle. Once within the threshold distance, the aerial robot has been slowed down by the DKB and eventually come to halt at the safety distance. Fig. 3.14 shows the time evolution of the translational movement along the $\mathrm{x}$-axis, where the shaded areas indicate the duration in which the DKB has been active. During the activation of the DKB, a clear separation of the operator's input force and velocity reference to the slave robot can be observed, which indicates that the operator is in contact with DKB because the aerial robot is approaching a potential obstacle; in the meantime, the velocity of the slave has also been decreasing and eventually brought to zero. It is worth noticing that, from 10 s to $15 \mathrm{~s}$, even when the robot arrives to the safety distance and the operator has deliberately given a forward flying command, the DKB successfully stopped the slave from further reaching the obstacle.

It can be clearly observed from the figure that the operator has been directly interacting with the virtual slave embedded in the master controller. This has enabled the operator to successfully teleoperate the aerial robot even in the presence of significant constant time delay. Attempts to perform similar experiments without the impedance reflection in the presence of the same time delay has proved to be 
difficult, illustrating the significance of the proposed approach.

\subsubsection{Impedance Framework}

In the next experiments, an impedance-based haptic tele-control architecture is used to perform internet-based applications. More specifically, the task is a navigation task that involves a human operator located at the University of Twente, The Netherlands, and a quadrotor-slave located at the Australian National University, Australia.

Intercontinental free-flight using the $\mathbf{P}-\mathbf{F}$ architecture This experiment shows the applicability of the impedance-based P-F architecture using the virtual slave concept (implemented only on the slave side), in the presence of variable time delays and significant packet losses. Fig. 3.15 shows satisfactory tracking of the scaled master position by the velocities of both the virtual and the real slave, despite the presence of time-varying delays shown in Fig. 3.16. Note that accurate and prolonged zero velocity commands seen in Fig. 3.15 are made possible due to the dead zone implemented around the neutral position of the impedance haptic interface.

Fig. 3.17 shows the haptic feedback displayed to the operator during the course of this test. The large transparency deviation shown in Fig. 3.18 is caused mainly by the non-negligible time-varying delays, and packet losses that account for $\approx$ $17 \%$ message loss. In the transparency analysis shown in the figure, transparency deviations due to non-zero inertia of the haptic interface and damping forces have not be counted for.

Intercontinental free-flight using the IR-I architecture This experiment is similar to the previous free-flight experiment using the impedance framework. However, in this case, the reflected impedance of the slave aerial robot is also embedded in the master controller. Similar to the IR-A case, the operator is able to tele-control the aerial with improved perception, even in the presence of variable time delays, see Figs. 3.19-3.22. The average packet loss registered in this experiment is $\approx 19.7 \%$. Similar to the previous experiment, transparency deviations due to non-zero inertia of the haptic interface and damping forces have not be counted for.

Maneuvering through a structure with obstacle avoidance In addition to the previous experiments illustrating the effectiveness of the control architecture presented in this chapter, the applicability of the control architecture presented in the previous chapter has also been demonstrated in an internet-based application. The task performed in this experiment is flying through the structure shown in Fig. 3.23. The operator maneuvers the aerial robot through the structure and avoids collision with the walls and the supporting poles with the aid of an obstacle avoidance algorithm. 

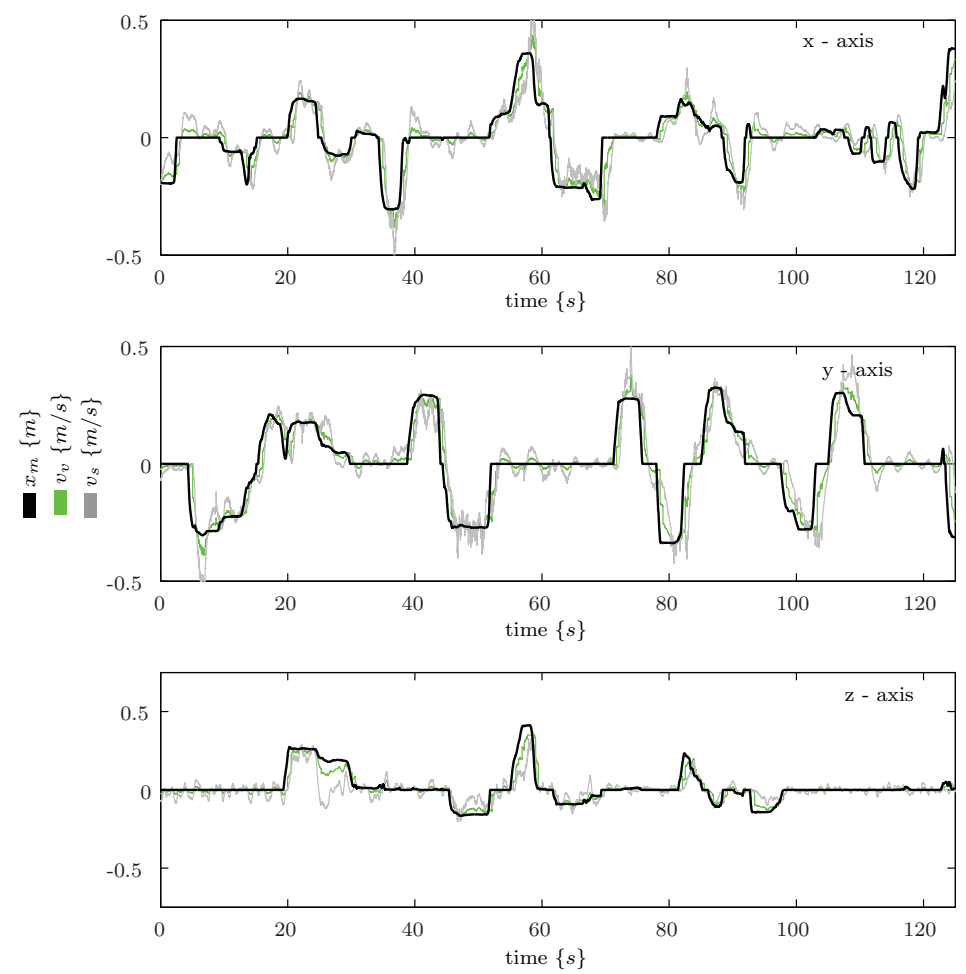

Figure 3.15: Scaled master position, velocity of the virtual and the real slaves, in the intercontinental test using the P-F architecture.
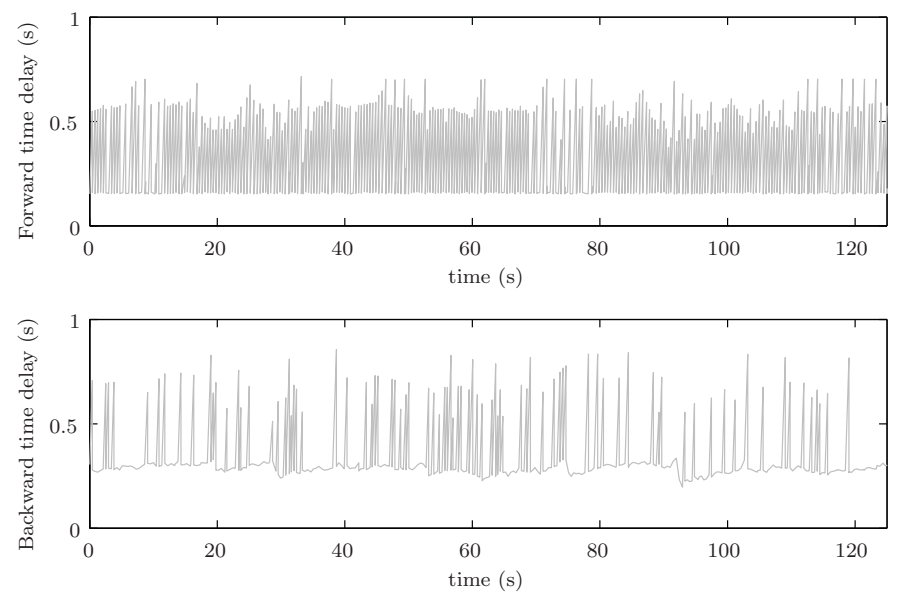

Figure 3.16: Communication channel delays during the course intercontinental test using the P-F architecture.

For obstacle avoidance, the low-level translational dynamics control of the aerial robot, particularly the $x$ and $y$, is augmented by obstacle avoidance forces. The obstacle avoidance force is based on the concept of optical flow that compares estimates of time-to-contact and maximum stopping time to produce a force that becomes noticeable only when the robots is performing a maneuver that may lead it to come close to collision. It is computed by [36].

$$
F_{\mathrm{ob}}=-\frac{k_{\mathrm{ob}}}{d_{\mathrm{ob}}} v_{s} \quad \text { if } d_{\mathrm{ob}}<d_{\mathrm{th}}
$$




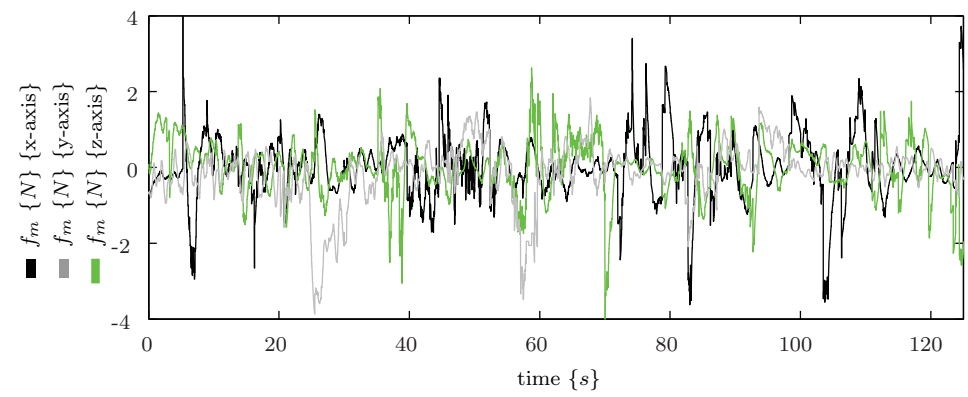

Figure 3.17: Force Feedback displayed to the operator during the course of the intercontinental test using the P-F architecture.

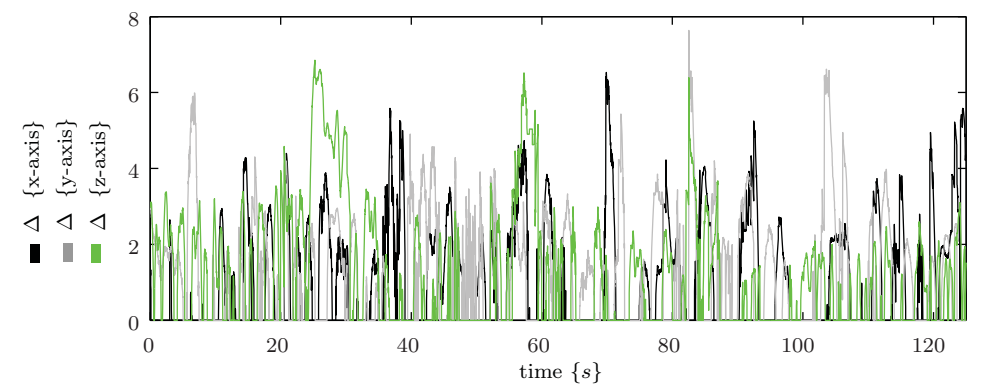

Figure 3.18: Transparency deviation in the intercontinental test using the P-F architecture.
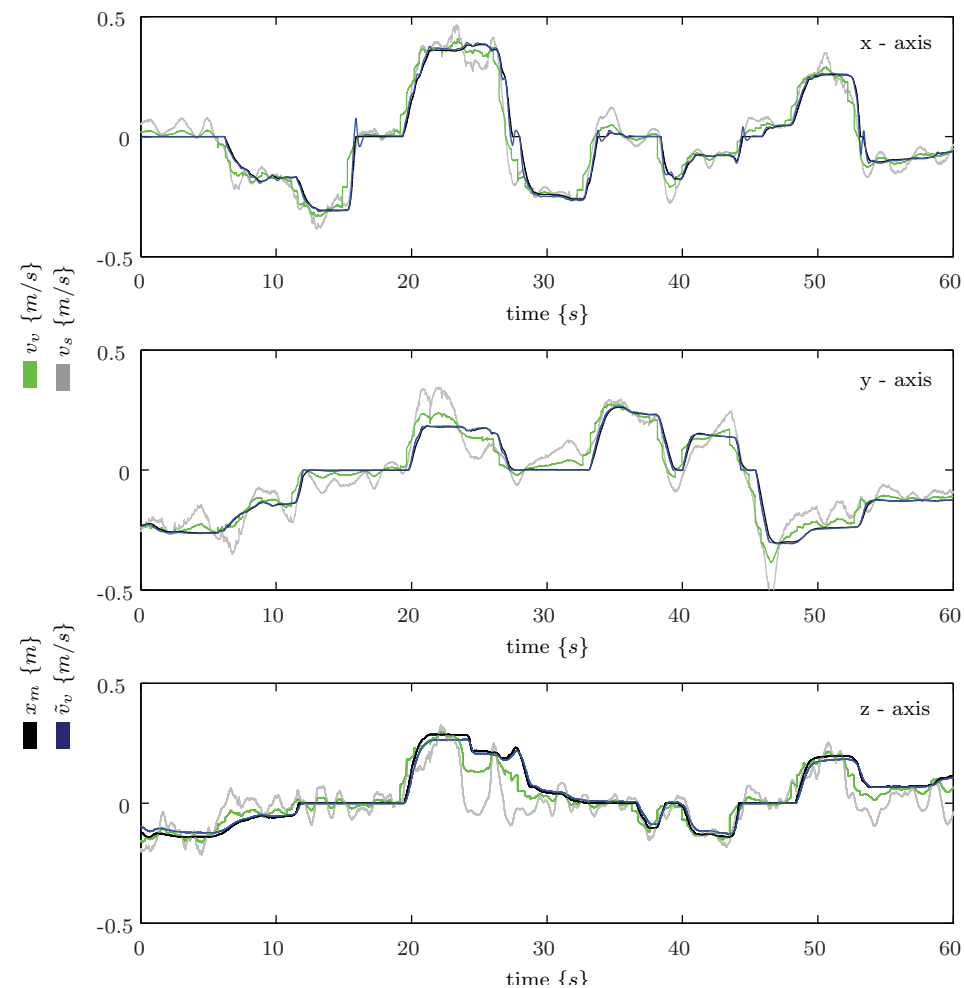

Figure 3.19: Scaled master position, velocity of the virtual and the real slaves, in the intercontinental test using the IR-I architecture.

where $k_{\mathrm{ob}}$ is a constant; $d_{\mathrm{ob}}$ is the displacement of the quadrotor from the obstacle, and $d_{\mathrm{th}}$ is a threshold, below which the obstacle avoidance force is applied.

The results of this experiment are depicted in Fig. 3.24-3.26. The command 

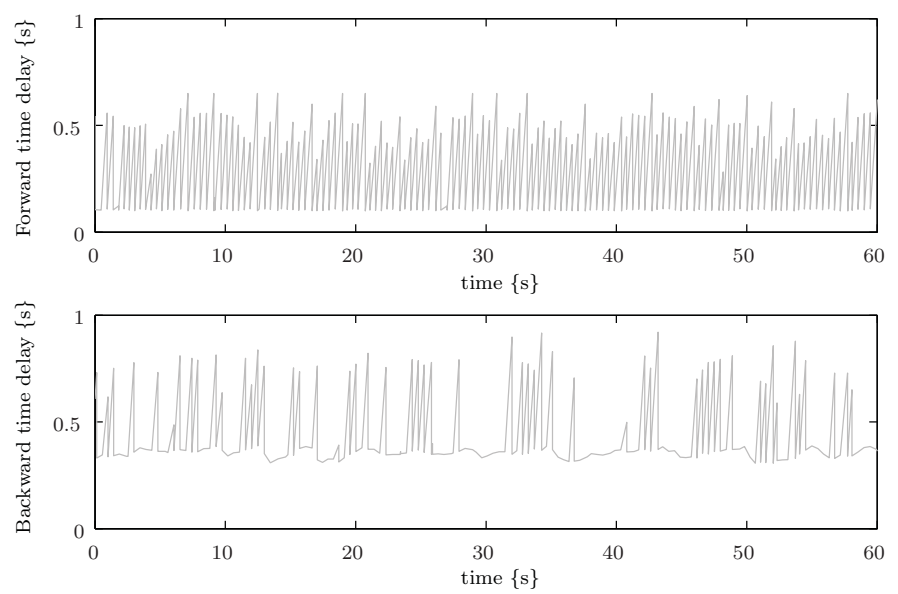

Figure 3.20: Communication channel delays during the course intercontinental test using the IR-I architecture.

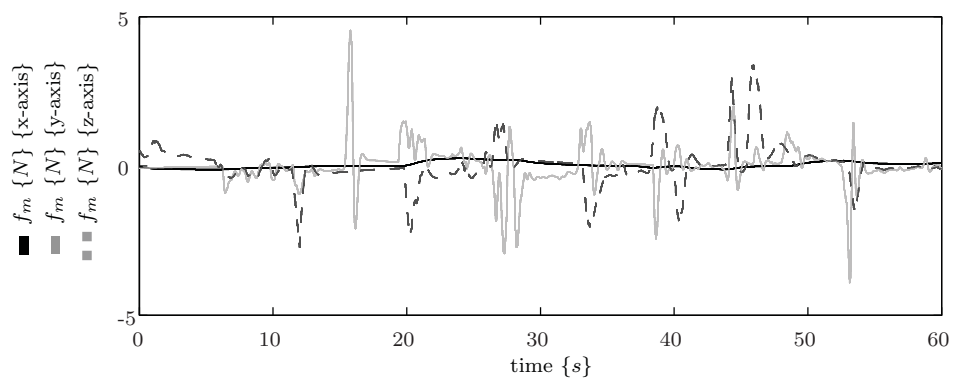

Figure 3.21: Force Feedback displayed to the operator during the course of the intercontinental test using the IR-I architecture.

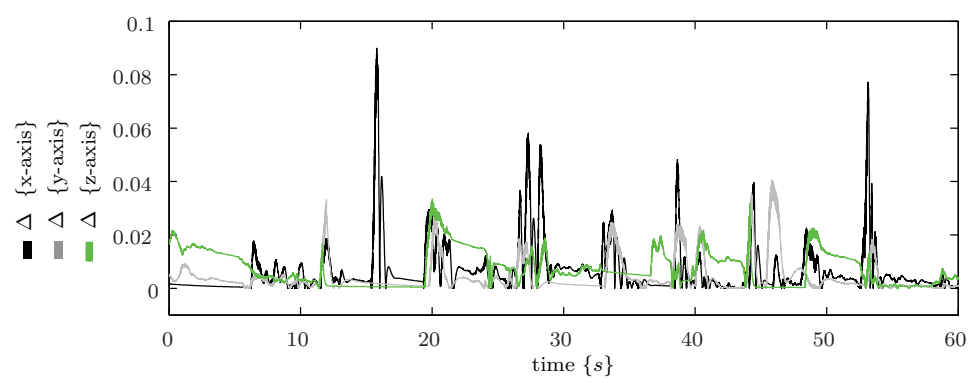

Figure 3.22: Transparency deviation in the intercontinental test using the IR-I architecture.

of the operator depicted is tracked by both slaves as shown in Fig. 3.24, until the obstacle avoidance force is applied on the robot. On the course of the experiment, the operator deliberately commanded the aerial towards the walls and poles. However, the obstacle avoidance force slows the robot down once it gets closer than 10 $\mathrm{cm}$. The environmental forces applied on the robot are also shown in Fig. 3.24 reduced by a scale of 10 for better comparison.

The deviation of the real slave and the virtual slave from the command during the application of the obstacle avoidance force is reflected back to the operator through the force feedback, see Fig. 3.25. The variable time delays registered in this experiment are depicted in Fig. 3.26. Average packet losses of $20.5 \%$ have been registered during this experiment. 


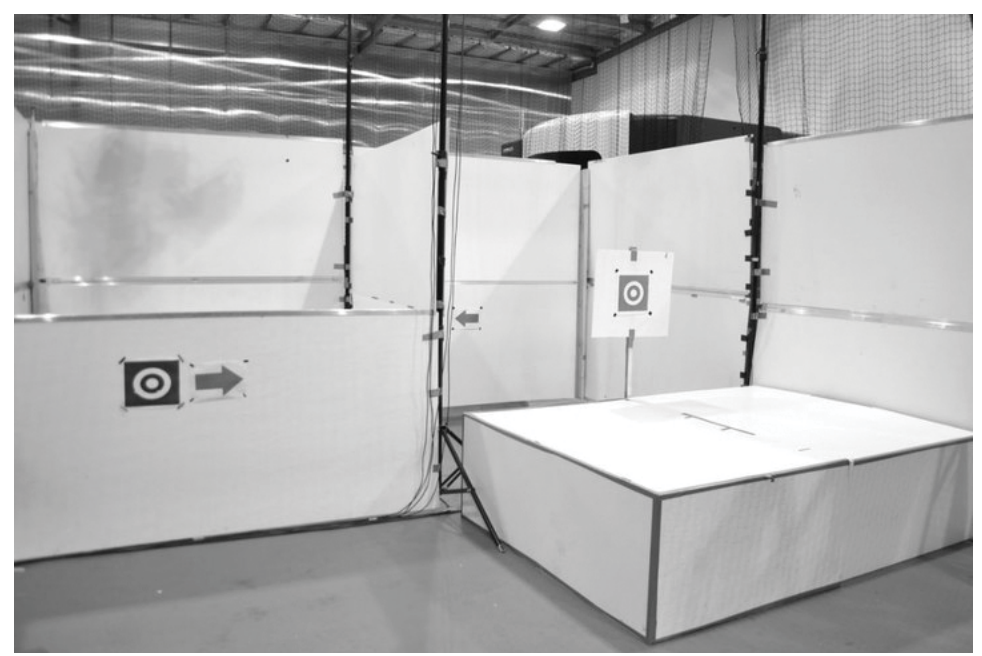

Figure 3.23: The structure through which the aerial robot is maneuvered without collision.

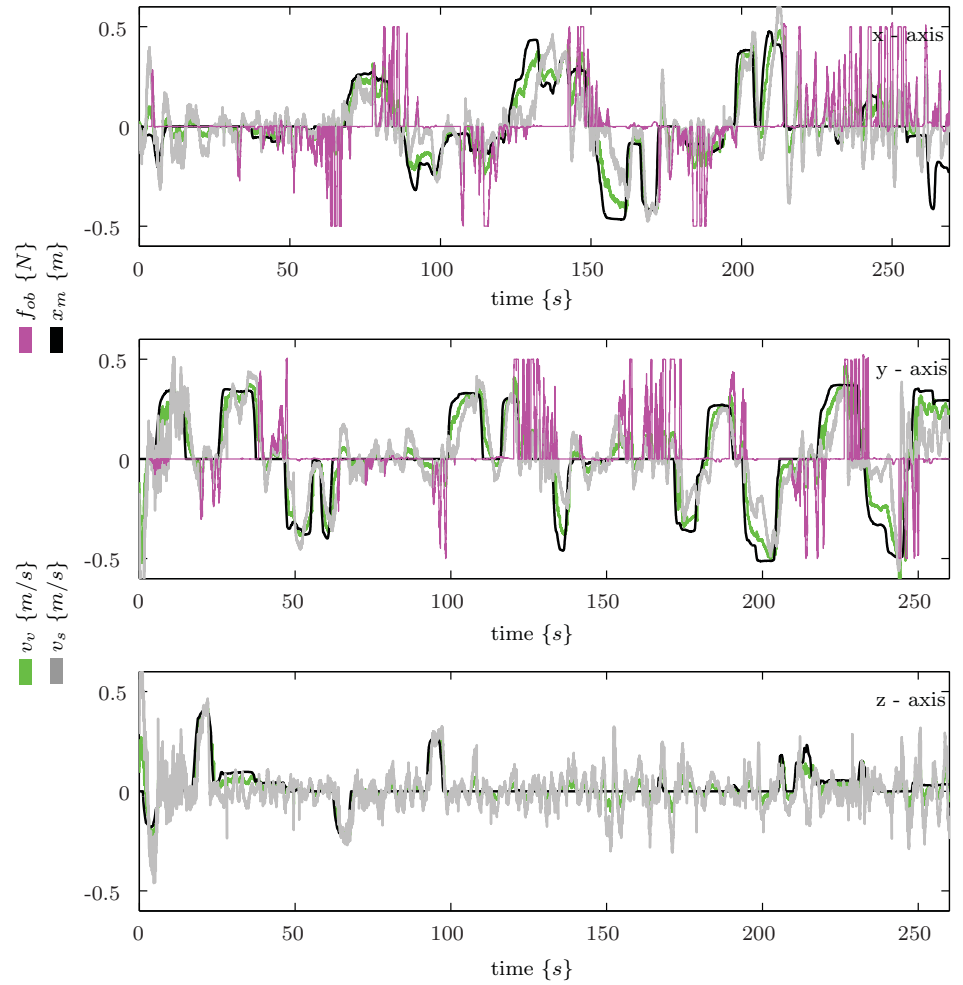

Figure 3.24: Plots of the scaled command of the operator, the velocities of the virtual and real slaves, and the scaled obstacle avoidance force.

\subsection{Discussion}

In this section, a general discussion based on the results obtained from the series of simulations and experiments are presented. Besides, a comparative evaluation of the admittance and the impedance control frameworks, with respect to their merits and practical implementations challenges, are provided. 


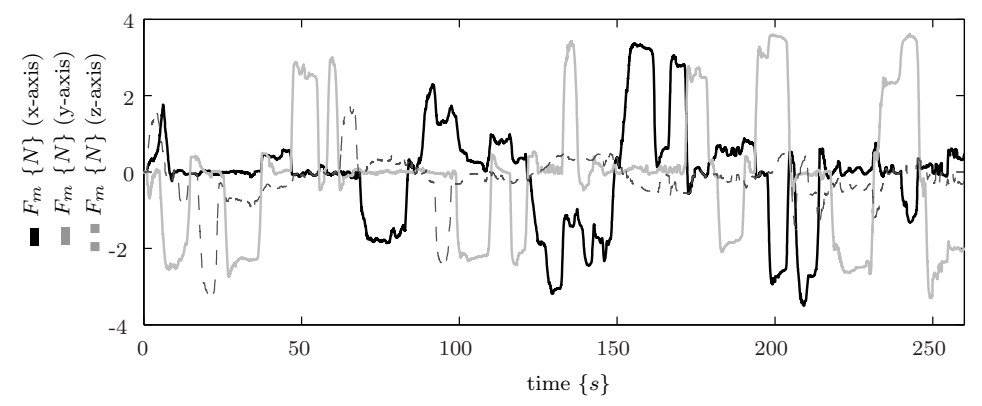

Figure 3.25: Haptic Feedbacks
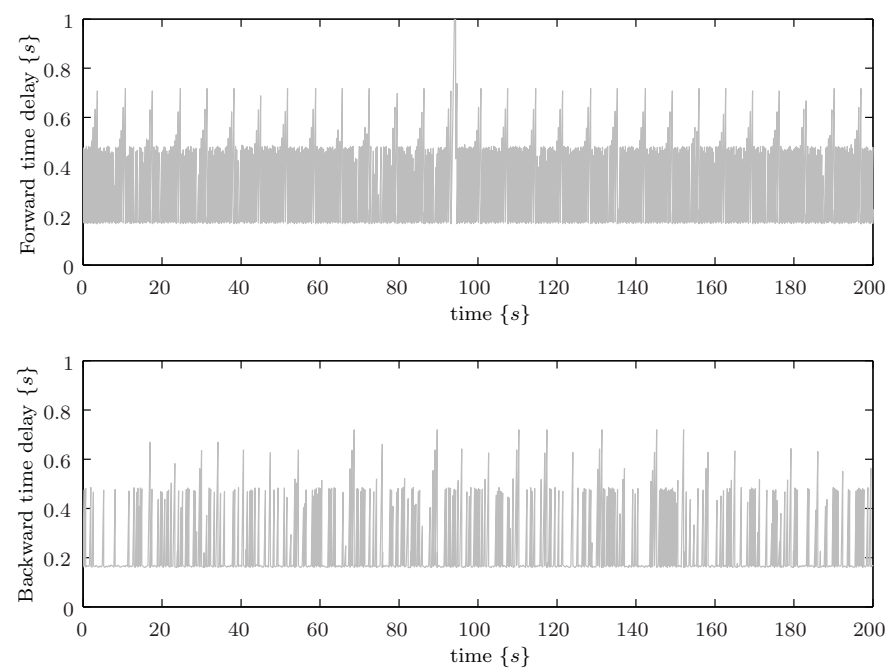

Figure 3.26: Time delays in the forward and backward traveling signals.

\subsubsection{General Comments on Results}

The simulations and experimental results demonstrate the significant improvement in the achievable transparency by using the proposed control architecture, in applications where the network-induced imperfections are non-negligible. Besides, they show the applicability of the proposed control architecture in both impedance and admittance frameworks.

The impedance reflection approach has enabled the operator to reliably interact with the remote slave/environment indirectly, through their identified model, which is embedded in the master controller. This, as a consequence, have greatly reduced the impact of network-induced imperfections, and have resulted in improved perception of the teleoperated slave/environment. This is primarily due to the time-wise collocation of the cause and effect at the operator's side. The results have also shown that the impedance reflection approach is significant in tele-control applications where the network-induced imperfections are non-negligible. In other applications, in which these imperfections are negligible, the approach's computational cost and delay due to identification process becomes more noticeable than its classical counterpart.

The DKB algorithm, on the other hand, has provided the operator with an in- 
tuitive kinesthetic feedback, resulting in environmental transparency. It has been shown that by dynamically changing the boundary of the haptic workspace, collision can be avoided even in the presence of time delay in the control loop. The DKB ensures collision avoidance by essentially bounding the velocity of the aerial robot smoothly in accordance with how closes it is to an obstacle. It has also been shown that obstacle avoidance can be achieved by augmenting the translational tracking controller with obstacle avoidance controller.

Furthermore, results have shown that avoiding switching for ease of implementation, has effects on the achievable transparency. To achieve a close to ideal vehicle and environment transparencies, switching is required based on the flight regime the aerial robot is in, i.e., free-flight or interaction.

In general, the proposed control architecture have shown to effectively circumvent the network-induced imperfections, leading to significantly improved transparency. Moreover, the DKB approach has enhanced the perception by providing distinct kinestethic cues, and has increased the task-performance by ensuring obstacle avoidance.

\subsubsection{General Comments on Practical Implementation of Ad- mittance and Impedance Based Haptic teleoperation of Aerial Robots}

The comparative comments provided in this section are mainly based on observations during simulations and practical realizations of the proposed control architecture. The comparison encompasses different aspects of each control architecture. Haptic Feedback during Free-flight: In impedance control architecture, the haptic feedback during a free-flight is the same as the force applied to the aerial robot, which is proportional to the deviation of the controlled state. In rate control, the controlled state is the velocity of the aerial robot. Hence, for velocity commands that fall within the bandwidth of the controller, the operator feels nonzero feedback when the aerial robot accelerates/decelerates. On the other hand, in the admittance architecture, any nonzero velocity command is felt by the operator as a reaction force, which is proportional to the velocity command. This notable difference between the two architectures is also analyzed in detail in [36].

Loosely speaking, in free-flight, the haptic channel is more extensively used in the admittance than the impedance framework. However, the fact that the force applied by the operator in the admittance framework is higher for the same motion profile of the slave also implies that it results in higher mechanical load to the operator than its impedance counterpart. This may stress the operator in free-flight teleoperation tasks that may take prolonged execution time.

Command Resolution and Precision: Unlike impedance haptic devices, admittance haptic devices do not require motion to detect the operator's input. As a result, the operator's force input, detected by admittance devices, has wider range than the operator's motion command, detected by impedance type haptic device. However, maintaining precise operator command is more difficult in admittance tele-control frameworks than impedance frameworks. 
Hovering Command: In rate-based impedance tele-control architecture, for hovering of the aerial robot, a zero velocity command is required. This command corresponds to the neutral position of the haptic interface, which is often difficult to manually detect. To overcome this limitation, a dead zone, which is easier to detect, is proposed in [71]. On the other hand, this is not an issue in admittance architecture, as releasing the haptic interface corresponds to a zero velocity command.

Intuitiveness: Interaction forces, such as obstacle avoidance and active interaction forces, are more naturally rendered in impedance tele-control architectures than admittance. In admittance frameworks, environmental forces are only perceived as added inertia.

Responsiveness: The response of the aerial robot is often faster in rate-based admittance tele-control architecture than its impedance counterpart. This is mainly due to the haptic interface and the employed mapping strategy. In admittance devices, sensing the operator's input force virtually does not require movement, whereas in impedance devices, it does. This motion in impedance devices, introduces delay in the response. Moreover, in position controlled aerial robots, rate-based tele-control essentially requires the integration of the position of the haptic device. This integration introduces additional delay in the response of the aerail robot, as perceived by the operator.

DKB Implementation and Perception: As show in Section 3.4.1 and noted in [34], implementation of the DKB requires additional contact dynamics to render a contact force in impedance framework. On the contrary, its implementation in admittance framework is direct. However, its perception in impedance framework is more intuitive. Moreover, the operator is in charge of the command modification in impedance framework, while the operator's input force is modified to ensure collision avoidance in admittance framework.

\subsection{Conclusion}

This chapter presented a tele-control architecture for aerial robots that enhances transparency in the presence of significant network-induced imperfections. First, since the classical definition of transparency only considers environment transparency, a general time-domain based transparency that also includes vehicle transparency has been defined. Mathematical evaluation of transparency deviation, including for time-delayed teleoperations, has been provided. In order to achieve improved transparency in the presence of network-induced imperfections, the passivitybased control architecture proposed in the previous chapter has been extended. The extensions include remote environment impedance reflection, and dynamic kinesthetic boundaries. The control architecture has been realized for the commonly used admittance and impedance tele-control frameworks.

The applicability and effectiveness of the proposed control architecture has been demonstrated in a series of simulations and experiments. The experiments included the longest intercontinental haptic teleoperation of aerial robots, which involved 
navigation of an aerial robot in Australian National University, Australia, from a master station at the University of Twente, The Netherlands. The simulations and experimental results demonstrated the improved transparency of the proposed control architecture in both control frameworks, with respect to the state of the art. Moreover, practical considerations that are necessary for effective utilization of the proposed architecture, and the hardware architecture that has been used to realize the longest intercontinental teleoperation of aerial robot have been presented. In addition, the merits and practical challenges of different aspects of impedance and admittance tele-control frameworks have been provided.

The presented definition of transparency, measure of transparency, and control architecture are general, hence, they can be applied to other fields of robotics as well. Moreover, the comparative evaluation of both admittance and impedance frameworks can be used as a basis for designing new controllers that combine the best features of each framework. Furthermore, the presented DKB approach can also be used for tasks that require active interaction, while ensuring smooth transition from free-flight to interaction, and limiting impact forces. 



\section{CHAPTER 4}

\section{Multimodal Tele-CONTRol architecture For AERIAL RoBOTS}

This Chapter presents a control architecture for haptic teleoperation of aerial robots involved in free-flight as well as active interaction. The goal of the control architecture is to enable the human operator to accomplish various types of tasks using a slave aerial robot, while achieving an improved telepresence through a multimodal human-machine interface. The slave control architecture is characterized by its unique capability of varying the apparent impedance of the controlled slave aerial robot as well as the interaction force, when in contact. Both the impedance and the interaction force are modulated by the human operator, based on the human operator's intention. The slave control algorithm also incorporates a slidingmode observer for state estimation, external force estimation, contact detection, and contact-point identification, which are required for both the impedance control and the regulation of the interaction force. The master control is also based on a variable impedance control architecture, and includes a sliding-mode observer to estimate the human operator's force. Moreover, a multimodal sensory feedback, which includes force and tactile feedbacks, is displayed to the operator to increase awareness of the remote environment, in order to increase the task-performance. The feasibility and effectiveness of the proposed control architecture are corroborated by extensive simulations and experimental results.

This chapter is an adapted composition of (Mersha et al. 2014c); A.Y. Mersha, S. Stramigioli, and R. Carloni, "Variable Impedance Control for Aerial Interaction", In Proceedings of the IEEE/RSJ International Conference on Intelligent Robots and Systems, 2014. Under review, and (Mersha et al. 2014d); A.Y. Mersha, M. Fumagalli, S. Stramigioli, and R. Carloni, "Multimodal Variable Impedance Control for Haptic Teleoperation of Interactive Aerial Robots", International Journal of Robotic Research, 2014. Under review. 


\subsection{Introduction}

Application of miniaturized unmanned aerial robots have been originally limited to tasks that do not involve active interaction with the environment, such as visionbased surveillance, forest fire detection, crop dusting, search and rescue [107]. However, recent technological advancement in the field of aerial robotics have shifted the focus of application to tasks that require active interaction with the environment, such as brushing, docking and sliding, power plant inspection by contact, aerial grasping, load transportation, and cooperative aerial assembling [59], [90], [87].

This paradigm shift has resulted in a wide range of potential application areas, where aerial robots can be considered. As the complexity of the tasks increases, the inclusion of the human in the control loop, either as a supervisor or a collaborator, becomes necessary or desirable. In such situations, teleoperation lends itself well to effectively accomplish complex tasks involving aerial interaction. However, it also poses interesting research challenges in controlling the often underactuated aerial robots in teleoperation setting, such as dealing with stability during a constrained motion, regulating time-varying interaction force, and ensuring stable and transparent interaction with the human operator.

\subsubsection{State of the Art}

In this section, literature in the field of aerial interaction and teleoperation of aerial robots that are relevant to the work presented here is briefly reviewed.

\subsubsection{Interactive Aerial Robots}

Few results have been reported in the field of aerial interaction so far. Hybrid position/force controller has been designed for a ducted-fan miniaturized aerial vehicle in [61]. In [3] and [102], similar controllers have been realized for a coaxial rotorcraft and a quadrotor endowed with a robotic manipulator, respectively. These hybrid controllers often rely on force/torque measurements obtained from on-board force sensors to detect contact and stabilize to a desired force set-point. Force sensors, besides their cost, additional dynamics, limited bandwidths and sensitivity to noise, limit the application scope due to small contact area, where the sensors are attached to. A hybrid controller has also been realized on a quadrotor in [7], where wrench estimator, instead of force sensor, is used. One of the limitations of this controller is the need for a priori choice of selection matrix that determines which DOFs are position or wrench controlled. In general, hybrid position/force controllers perform well in environments that are well-structured, and when the coupling between the position and force controlled DOFs are known.

A step toward achieving safety in the manipulation of unknown objects or interaction with unknown environment includes guaranteeing a stable interaction, even during an unexpected contact. In [81], a blind navigation controller that ensures robust stability, including during an unexpected contact, has been proposed. 
The pioneering work of Hogan on impedance control [32] has been widely used in interaction with unknown, unstructured and dynamically changing environments. Impedance control, unlike explicit force and position controllers, specifies the desired dynamic behavior between force and position [109]. In the field of aerial robotics, impedance control has been implemented on aerial manipulator in [21], [26]. Though interaction is only realized through the end-effector of the manipulator mounted on the aerial robot, results show that the impedance controller effectively handles the transition between free-flight and docking. In [25], a modified impedance control based on virtual mass has been proposed.

The main limitation of classical impedance controllers is their fixed impedance. Various tasks require different impedance behavior to attain a desired level of task performance. For instance, precise position tracking requires stiff dynamics, while probing or docking on unstructured and unfamiliar environment requires compliant dynamics, much like humans. Thus, no single impedance is optimal for every application [117], [8]. On the other hand, as force control is not often the goal of classical impedance controllers, it is difficult, if not impossible, to regulate interaction force when the position of the environment is not known a priori. Methods suggested in the literature to mend this limitation of impedance controllers mainly involve varying the position command [105], [95], or the stiffness of the impedance controller [55], [19]. The aforementioned works, use force sensors and regard one dimensional system. Moreover, most of them illustrate the applicability of the control algorithms in simulations.

\subsubsection{Teleoperation of Aerial Robots}

Although teleoperation is a field which has been researched for the past half century, its application to aerial robots is fairly new [58], [47], [111]. Recently, haptic teleoperation of aerial robots has been used for free-flight, obstacle avoidance, and target attraction tasks [48], [58], [10]. The proposed control frameworks include both admittance and impedance frameworks [36], [69], for single and multiple aerial robots [97], [22].

The research in haptic teleoperation of aerial robots have so far focused on addressing classical and unique challenges for navigation tasks that include obstacle avoidance [71], [36]. Unique challenges in haptic teleoperation of flying robots include underactuatedness of the aerial robots, workspace incompatibility between the master and the slave, and continuous energy dissipation even near hovering. The classical challenges are mainly due to network-induced imperfections such as time-varying delays and packet losses [67].

During navigation while avoiding obstacles, the haptic feedback indicates how close the approaching obstacles are with respect to the aerial robot. The various haptic-based obstacle avoidance algorithms differ only in the type of haptic feeback (resistive or repulsive) and the type of sensory data based on which they generate the obstacle avoidance force (vision, laser, telemetric sensors). Additionally or alternatively, the haptic feedback is used as an indication of the deviation of the current controlled state of the aerial robot with respect to the command. 


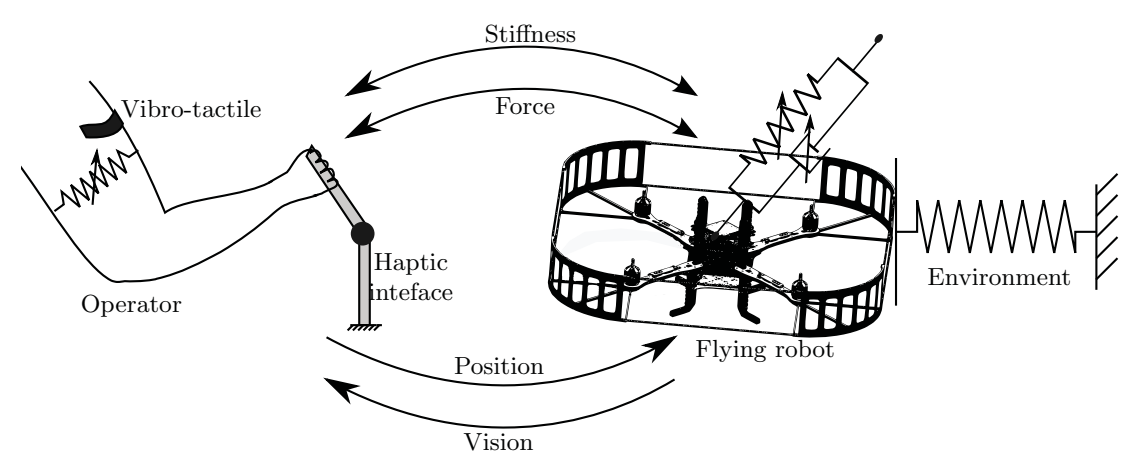

Figure 4.1: Conceptual representation of the proposed tele-control architecture. It shows a human operator commanding the desired impedance, force and configuration trajectories of the slave aerial robot, while obtaining vision, force, impedance feedbacks through human-machine interfaces constituting the complete master system.

The literature still lacks a control architecture for haptic teleoperation of aerial robots that are involved in active interaction. A step forward is to project the capabilities of classical bilateral teleoperation robots to teleoperation of interactive aerial robot. In an attempt to re-create direct interaction between the operator and manipulated object/environment, classical teleoperation algorithms have relied on the exchange of position and/or force signals between the master and the slave. Although significant achievements have been made using such tele-control algorithms, there is still a long way to go to achieve human-level manipulation capabilities.

\subsubsection{Contribution}

The main contribution of this work is a versatile bilateral teleoperation control architecture that is capable of varying the impedance of the controlled aerial robot and regulating time-varying interaction force, when contact is detected. The impedance of the controlled aerial robot is explicitly modulated based on the upper arm muscular activities of the human operator. Moreover, a multimodal feedback is displayed to the operator to increase the situational awareness of the aerial robot and/or environment. Fig. 4.1 shows the conceptual representation of the proposed tele-control architecture.

The slave controller is characterized by an inner-loop variable impedance controller and an outer-loop that modifies the impedance, when interaction is detected, in order to regulate the interaction force. This outer-loop controller is now on called "force controller", but in the context of impedance regulation, not as pure force controller. The force controller relies on a robust nonlinear sliding-mode observer for state estimation, external force estimation, contact detection, and contact-point identification.

The master controller, on the other hand, realizes a variable impedance behavior of the haptic interface that is responsible for displaying force feedback to achieve transparency. Besides force and vision feedback, vibrotactile feedback that corresponds to the impedance of the slave or the environment is displayed to the 


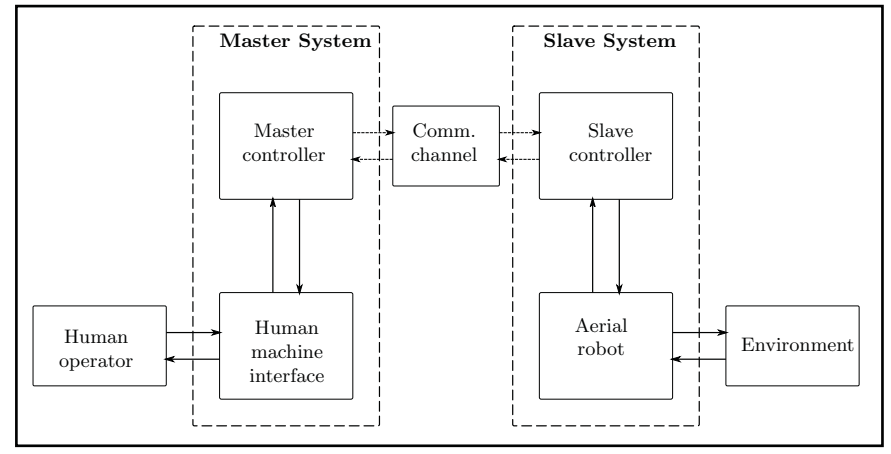

Figure 4.2: A generic bilateral tele-control scheme of aerial robots, composed of the seven most common functional blocks. Note that the block commonly representing "haptic interface" is replaced by "human-machine interface" to emphasize that the feedback to the operator is not only haptic but also other multimodal sensory feedback, including vision and tactile. Moreover, the operator's input measured by the human-machine interface also includes EMG signals, which are used for derivation of the desired impedance of the controlled aerial robot.

operator. A sliding-mode observer is also included in the master controller to estimate the states of the haptic interface and the operator's input force.

This work finds its way in various applications where different impedance and interaction force control provide higher performance as well as safety. Some of the applications include inspection of plants by applying a desired force, docking on a structure (including movable), physical interaction and cooperation with other aerial robots, and accidental but safe interaction with an environment (including humans).

The rest of the Chapter is structured as follows. Section 4.2 provides a brief background on the teleoperation control architecture, and benchmark models of the slave aerial robot and the haptic interface. Sections 4.3 and 4.4 provide the main contributions of this work, i.e., the variable impedance and the interaction force tracking slave controller, and the multimodal feedbacks to the operator, along with their rigorous mathematical analysis. Section 4.5 provides useful practical considerations for effective utilization and realization of the control architecture. Simulations and experimental results demonstrating the effectiveness of the proposed algorithm are given in Sections 4.6 and 4.7. Concluding remarks are given in Section 4.8 .

\subsection{Background}

In this section, a background on the general tele-control architecture, and the dynamic models of the slave aerial robot and the haptic interface are presented.

\subsubsection{Tele-control Architecture}

The tele-control architecture of the interactive aerial robot is similar to the classical bilateral teleoperation scheme composed of seven functional blocks, see Fig. 4.2. 
The architecture is composed of software and hardware components that enable bilateral communication between the human operator and the remote environment. The human-machine interface, which is composed of haptic interface, graphical user interface, vibrotactile motors and Electromyography (EMG) reader, is responsible to read the operator's intent and feed back the reaction of the slave and/or the environment to the operator's stimulus. The master controller elaborates the sensory data representing the operator's intent and communicates them to the slave controller, which is responsible for realizing the operator's intent by modifying the behavior of the slave robot, which is either in free-flight or directly interacting with the environment. The master controller also generates appropriate feedback to the operator to create situational awareness of the remotely teleoperated slave robot or environment.

As described in Section 4.1.2, the main goal of this work is to design and realize a flexible bilateral tele-control architecture with a user-modulated variable impedance and interaction force regulation without force measurement capabilities. Thus, the main focus blocks in the development are the master and the slave controllers, which are responsible to realize the proposed capabilities. Networkinduced imperfections, such as variable time delays and packet losses are not treated here. Interested readers are referred to Chapters 2 and 3, and references therein.

\subsubsection{Modeling}

To limit the mathematical complexity, planar dynamic models of both the slave aerial robot and the haptic interface are used as running examples to design their respective controllers. In fact, the proposed controller architecture is applicable mainly to any multidimensional aerial robot with VTOL capabilities, and any multidimensional haptic interface. The applicability of control algorithm for other aerial platforms, such as ducted-fans, is also demonstrated in Section 4.6. Moreover, its practical applicability is experimental demonstrated for a $3 \mathrm{D}$ aerial robot in Section 4.7.

\subsubsection{Notation}

With respect to Fig. 4.3,

- $\psi^{i}=\left[o_{i}, \overrightarrow{\hat{x}_{i}}, \vec{y}_{i}\right]$ denotes a right handed orthonormal coordinate frame $i . \psi^{0}$ and $\psi^{u}$ represent the inertial coordinate frame, and the body-fixed frame attached to the COM and aligned with the principal axis of the aerial platform. $\psi^{c}$ denotes the contact coordinate frame, whose $y$-axis is normal to the contacting surface.

- $R_{j}^{i} \in \mathbb{R}^{2 \times 2}$ is an orthonormal rotation matrix describing the orientation of $\psi^{i}$ with respect to $\psi^{j}$, and it also transforms coordinates from $\psi^{j}$ to $\psi^{i}$.

- $p_{j}^{i}=\left[\begin{array}{ll}x_{j}^{i}, & y_{j}^{i}\end{array}\right]^{T} \in \mathbb{R}^{2 \times 1}$ is a vector describing the displacement of the origin of $\psi^{j}$ from the origin of $\psi^{i}$ in coordinates of $\psi^{i}$. 


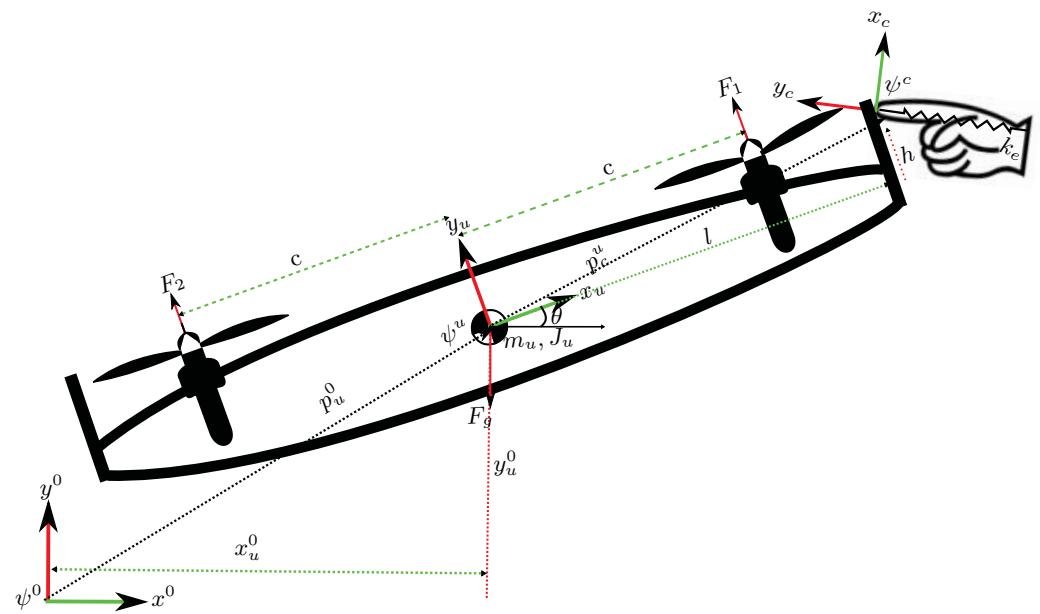

Figure 4.3: Illustration of a planar quadrotor interacting with an environment, which is characterized by a predominantly spring characteristics. The figure also shows the notations used throughout this Chapter. Note that the $y$-axis of $\psi^{c}$ is normal to the contacting surface, it is not necessarily aligned with the $x$-axis of $\psi^{u}$ or $x$-axis of $\psi^{0}$.

- $m_{u}$ and $J_{u}$ denote the mass and the rotational inertia of the planar aerial robot.

- $F_{1}$ and $F_{2}$ represent the actuation forces due to the propellers; $F_{g}$ and $F_{\text {ext }}$ represent the gravitational and the interactions forces, respectively.

- $c \in \mathbb{R}^{+}$is a nonzero constant lateral displacement of each propeller from $\psi^{u}$.

Moreover, for a generic vector $\kappa, \kappa[i]$ represents its $i^{\text {th }}$ element.

\subsubsection{Dynamic Model of an Aerial Robot}

Most commercially available VTOL aerial robots are characterized by fully actuated rotational dynamics and underactuated translational dynamics, which is also captured by the planar aerial robot considered in this work. With reasonable assumption of negligible aerodynamic effect in low velocity flights, their dynamics is given by a single planar rigid body in the planar special Euclidean configuration space $S E(2):=\left\{S O(2) \times \mathbb{R}^{2}\right\}$, where $S O(2)$ is the Lie group indicating the set of planar orthonormal rotational matrices. Various forces, including those due to actuation, gravity and contact, act on this planar aerial robot. To simplify the control design process, the following assumptions are made.

Assumption 4.1 Even though the actuation strategies in different platforms, such as ducted-fan, may induce small body forces, they are negligible or can be compensated by controller action. ${ }^{1}$ Hence, the actuator input $u \in \mathbb{R}^{3}$ of an underactuated

\footnotetext{
${ }^{1}$ Although small body forces and aerodynamically induced forces are assumed to be either negligible or compensated by controller action, the applicability, thereby, robustness of the proposed algorithm is demonstrated in simulation by incorporating those forces in the model (see Section 4.6). Besides, they are inevitably present during experimental realization (see Section 4.7).
} 
VTOL aerial robot, composed of the total thrust of the propellers and their net torque, has the following form,

$$
u^{T}=\overbrace{\left[\begin{array}{ll}
0 & F_{1}+F_{2}
\end{array}\right.}^{f_{\text {input }}^{u}} \overbrace{c\left(F_{1}-F_{2}\right)}^{\tau_{\text {input }}^{u}}]
$$

Assumption 4.2 A single interaction point between the aerial robot and the environment is considered. Furthermore, interaction is assumed to occur at the side periphery of the robot. Due to the specific choice of $\psi^{c}$, the interaction force $f_{\text {ext }}^{c}$ has the following form

$$
\left(f_{\text {ext }}^{c}\right)^{T}=\left[\begin{array}{ll}
0 & F_{\text {ext }}^{c}
\end{array}\right]
$$

Assumption 4.3 The environment is assumed to be predominantly characterized by a linear spring. ${ }^{2}$ The contact dynamics is given by

$$
F_{e x t}^{c}=k_{e} \Delta x^{c}=k_{e}\left(x_{*}^{c}-x_{e}^{c}\right)
$$

where $F_{e x t}^{c}$ denotes the force applied on the environment by the aerial robot; $k_{e}$ represents the environmental stiffness; $\Delta x^{c}$ denotes the penetration during interaction, in which $x_{*}^{c}$ and $x_{e}^{c}$ denote the position of the aerial robot during the occurrence of contact and the position of the environment, respectively, both along the $y$-axis of $\psi^{c}$.

Based on the above assumptions and following [66], the translational dynamics is given by

$$
m_{u} \ddot{p}_{u}^{0,0}=-D_{t} \dot{p}_{u}^{0,0}+f_{\text {input }}^{0}+f_{g}^{0}-f_{\text {ext }}^{0}
$$

where $D_{t}=\operatorname{diag}\left(\left[\begin{array}{ll}d_{x} & d_{y}\end{array}\right]\right)$, is the viscous damping coefficients; $f_{\text {input }}^{0}=R_{u}^{0} f_{\text {input }}^{u}$ is the total thrust of the propellers projected along $\psi^{0} ; f_{g}^{0}=\left[\begin{array}{ll}0 & F_{g}\end{array}\right]^{T}$ is the gravitational force, and $f_{\text {ext }}^{0}=R_{c}^{0} f_{\text {ext }}^{c}$ is the interaction force vector. The rotational dynamics is given by

$$
J_{u} \dot{\omega}_{u}^{u, 0}=-d_{\omega} \omega_{u}^{u, 0}+\tau_{\text {input }}^{0}-\tau_{\text {ext }}^{0}
$$

where $d_{\omega}$ is the viscous damping coefficient; $\tau_{\text {input }}^{u}$ is the actuator input, and $\tau_{\text {ext }}^{u}=$ $\left(p_{c}^{u}\right)^{T} S^{T}(1) R_{c}^{u} f_{\text {ext }}^{c}$ is the external torque, in which $S(1):=\left[\begin{array}{cc}0 & -1 \\ 1 & 0\end{array}\right]$.

\footnotetext{
2 In fact pure stiffness is the worst passive environment in terms of coupled stability (aerial robot + environment) [13]. Loosely speaking, it reduces the modulus margin of the coupled system more than its damped counterpart, and it even can sometimes lead to instability of the coupled system.
} 


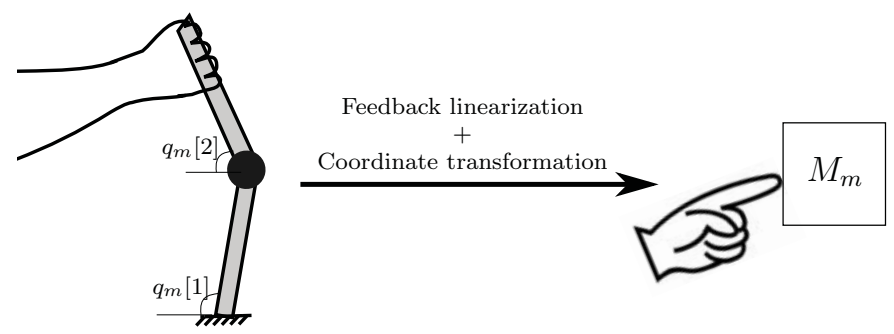

Figure 4.4: Illustration of the transformation of the dynamics of the haptic interface, as described by (4.6) and (4.7).

\subsubsection{Dynamic Model of a Haptic Interface}

The haptic interface is the principal component of the master system shown in Fig. 4.2. Impedance type haptic interface, which is considered in this work, is capable of sensing the position command of the operator and displaying force feedback. Often they are fully actuated, and their joint space dynamics is described by

$$
m_{m}\left(q_{m}\right) \ddot{q}_{m}+C_{m}\left(q_{m}, \dot{q}_{m}\right) \dot{q}_{m}+G_{m}\left(q_{m}\right)=\tau_{m}+J^{T}\left(q_{m}\right) f_{h}
$$

where $q_{m}$ denotes the joint space configuration; $m_{m}\left(q_{m}\right), C_{m}\left(q_{m}, \dot{q}_{m}\right)$ and $G_{m}\left(q_{m}\right)$ denote the generalized mass matrix of the haptic interface, the Coriolis and centrifugal matrix, and gravitational force vector, respectively. $\tau_{m}$ is the actuator input, which is responsible to both compensate for undesired parasitic dynamics and render the desired force feedback. $f_{h}$ is a Cartesian space force applied by the operator, which is transformed to its joint space equivalent through the Jacobian $J\left(q_{m}\right)$.

The most common point of interaction between the haptic interface and the operator is the tip of the haptic interface. By proper coordinate transformation and feedback linearization, [78], [69], (4.6) can be transformed to its equivalent in Cartesian coordinates, see Fig. 4.4. It dynamics in Cartesian space is given by

$$
m_{m} \ddot{p}_{m}^{0,0}=-d_{m} \dot{p}_{m}^{0,0}+f_{h}^{0}+f_{f b}^{0}
$$

where $p_{m}^{0}$ is the position of the tip of the haptic interface with respect to $\psi^{0} ; m_{m}$ and $d_{m}$ are the mass and damping parameters of the controlled haptic interface; $f_{h}$ and $f_{f b}$ denote the force applied by the human operator, and the force feedback, respectively.

\subsection{The Slave Control Architecture}

Due to the underactuation of aerial robots, often a cascaded control structure is employed to control the underactuated translational dynamics at the expense of some of the rotational DOFs. In this planar case, the translational dynamics along the $x$-axis is controlled at the expense of the pitch dynamics, i.e., the fully actuated pitch dynamics is controlled in such a way that a desired dynamics along $x$-axis is attained. The rotational dynamics of the aerial robot given in (4.5) is 


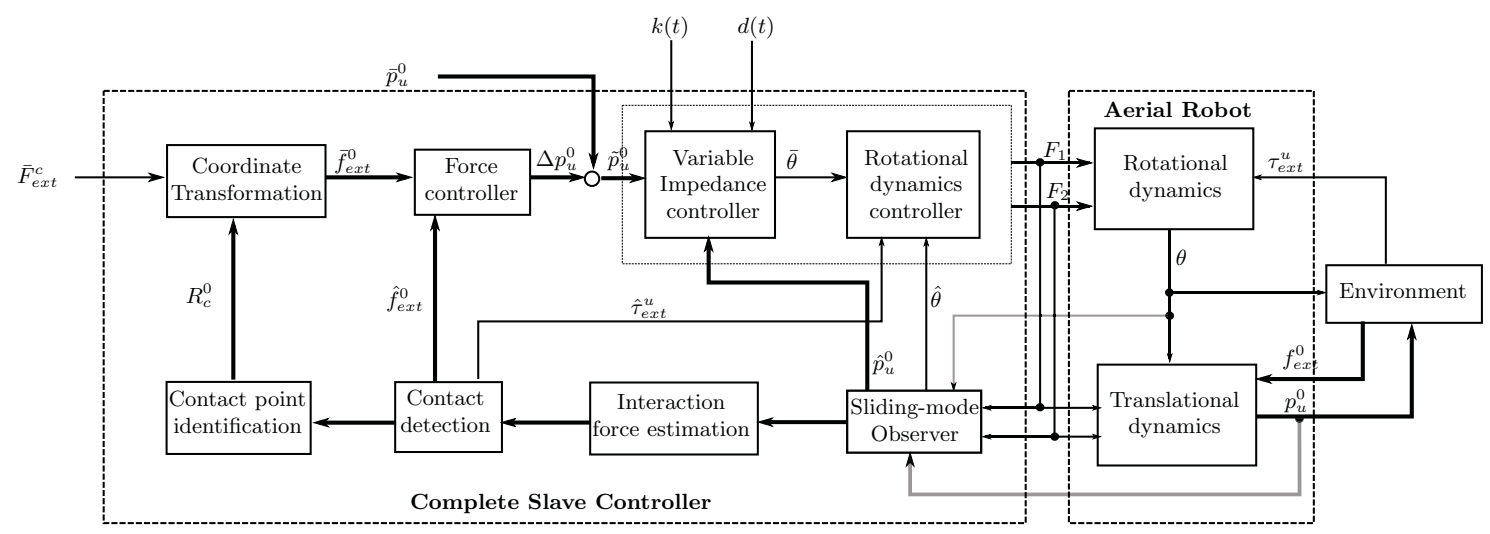

Figure 4.5: Block diagram representation of the slave controller, which is composed of an innerloop variable impedance controller and outer-loop force controller. The architecture shows the modulation of the stiffness, damping, position, and interaction force of the slave aerial robot. Note that the position and the interaction force of the slave are not simultaneously modulated as that is not possible. $F_{e x t}^{c}$ is modulated by the operator only when interaction is detected, otherwise, $\bar{p}_{u}^{0}$ is commanded by the operator. The gray signals indicate measurement data.

fully actuated and, with reliable estimation of the external torque $\tau_{\text {ext }}^{u}$, robust exponential stability can be attained [37]. The design of the pitch dynamics control is not the focus of this Chapter, and therefore, it is not treated any further. Interested readers are referred to, among others, [66], [37], [80] and Appendix A. Based on this consideration, $\theta$, along with $F_{1}+F_{2}$, i.e., $f_{\text {input }}^{0}$, is considered as a control input to the translational dynamics given in (4.4).

Fig. 4.5 shows the proposed control architecture of the slave robot, whose details are provided in the next sections.

\subsubsection{Variable Impedance Controller}

In this section, a variable impedance controller for the translational dynamics of the aerial robot is proposed. The main objective of impedance controller is to establish a certain dynamic relationship between the external force and position. The desired impedance is often a second order mass-spring-damper system given by

$$
m(t) \ddot{e}_{p}+d(t) \dot{e}_{p}+k(t) e_{p}=-f_{e x t}^{0}
$$

where $e_{p}:=p_{u}^{0}-\tilde{p}_{u}^{0}$ denotes the position error, in which $\tilde{p}_{u}^{0}$ is the virtual position to the impedance controller; $m(t), d(t), k(t) \in \mathbb{R}^{+}$represent the desired mass, damper and stiffness, respectively.

Note that $\tilde{p}_{u}^{0}$ is referred as virtual position to distinguish it from position command that is, in general, used in the context of a position control. In fact, in the absence of external force, the virtual position is the same as the position command, and tracking the virtual position is the goal of the impedance controller. However, during interaction, staying away from the virtual position in order to apply a force is part of the goal of the impedance controller. In this work, $\tilde{p}_{u}^{0}$ is the composition of the position command $\bar{p}_{u}^{0}$ and the modification due the action of the force 
controller $\Delta p_{u}^{0}$ (see Section 4.3.2) i.e.,

$$
\tilde{p}_{u}^{0}=\bar{p}_{u}^{0}+\Delta p_{u}^{0}
$$

To achieve the desired impedance, as given in (4.8), the control input to (4.4) is

$$
f_{\text {input }}^{0}=\left(m_{u}-m(t)\right) \ddot{p}_{u}^{0,0}+m(t) \ddot{\tilde{p}}_{u}^{0,0} d(t) \dot{e}_{p}-k(t) e_{p}+D_{t} \dot{p}_{u}^{0,0}-f_{g}^{0}
$$

In this study, only the stiffness $k(t)$ of the desired impedance is modulated by the operator based on noninvasive sensory measurement of the muscular activity of the operator (see Section 4.4.1). While keeping the target inertia $m(t)=m_{u}$, the damping parameter $d(t)$ is varied according to $k(t)$ to obtain a critically damped response. If the desired relative damping ratio is $\xi$, then the damping is tuned as

$$
d(t)=d(k(t))=2 \xi \sqrt{m_{u} k(t)}
$$

To ensure smooth adaptation of the variable stiffness and damping parameters of the desired impedance, the following adaptation laws are employed:

$$
\begin{gathered}
\dot{k}=-\alpha_{k}(k-\bar{k}) \\
\dot{d}=\dot{\bar{d}}-\alpha_{d}(d-\bar{d})
\end{gathered}
$$

where the bar indicates the reference values of the stiffness and damping, and $\alpha_{k}, \alpha_{d} \in \mathbb{R}^{+}$are constant gains.

Proposition 4.1 The variable impedance controlled aerial robot, whose dynamics is given by (4.4), (4.10)-(4.13) is an input to state stable (ISS) system with input $f_{\text {ext }}^{0}$ and $\bar{k}$.

Proof: Consider a candidate ISS-Lyapunov function $V=\frac{1}{2} m_{u} \dot{e}_{p}^{T} \dot{e}_{p}+\frac{1}{2} k e_{p}^{T} e_{p}+$ $\frac{1}{2} e_{d}^{T} e_{d} \geq 0$, for $e_{d}:=d-\bar{d}$. The time derivative of the $V$ results in

$$
\begin{aligned}
\dot{V} & =m_{u} \dot{e}_{p}^{T} \ddot{e}_{p}+k e_{p}^{T} \dot{e}_{p}+\frac{\left\|e_{p}\right\|^{2}}{2} \dot{k}+e_{d} \dot{e}_{d} \\
& =-d\left\|\dot{e}_{p}\right\|^{2}-\alpha_{k} \frac{\left\|e_{p}\right\|^{2}}{2} k-\alpha_{d} e_{d}^{2}+\alpha_{k} \frac{\left\|e_{p}\right\|^{2}}{2} \bar{k}-\dot{e}_{p}^{T} f_{\text {ext }}^{0}
\end{aligned}
$$

Let $0<\vartheta<1$, then,

$$
\begin{aligned}
\dot{V} & =-d\left\|\dot{e}_{p}\right\|^{2}+d \vartheta\left\|\dot{e}_{p}\right\|^{2}-d \vartheta\left\|\dot{e}_{p}\right\|^{2}-\alpha_{k} \frac{\left\|e_{p}\right\|^{2}}{2} k+ \\
& +\alpha_{k} \frac{\left\|e_{p}\right\|^{2}}{2} \vartheta k-\alpha_{k} \frac{\left\|e_{p}\right\|^{2}}{2} \vartheta k-\alpha_{d} e_{d}^{2}+\alpha_{k} \frac{\left\|e_{p}\right\|^{2}}{2} \bar{k}-\dot{e}_{p}^{T} f_{e x t} \\
& =-d(1-\vartheta)\left\|\dot{e}_{p}\right\|^{2}-\alpha_{k} \frac{\left\|e_{p}\right\|^{2}}{2} k(1-\vartheta)-\alpha_{d} e_{d}^{2} \\
& -\alpha_{k} \frac{\left\|e_{p}\right\|^{2}}{2}(\vartheta k-\bar{k})-\dot{e}_{p}^{T}\left(d \vartheta \dot{e}_{p}+f_{e x t}^{0}\right) \\
& <-d(1-\vartheta)\left\|\dot{e}_{p}\right\|^{2}-\alpha_{k} \frac{\left\|e_{p}\right\|^{2}}{2} k(1-\vartheta)-\alpha_{d} e_{d}^{2}<0
\end{aligned}
$$

provided that

$$
k>\frac{1}{\vartheta} \bar{k} \text { and }\left|\dot{e}_{p}\right|>\frac{1}{d \vartheta}\left|f_{\text {ext }}^{0}\right|
$$

The result of the above proof can be interpreted as follows. 
- For system trajectories fulfilling (4.15), $\dot{V}$ is negative definite, implying that the system trajectories approach the ball of radius $r:=\frac{1}{\vartheta} \sqrt{\bar{k}^{2}+\frac{1}{d^{2}}\left\|f_{\text {ext }}^{0}\right\|}$.

- If the system trajectories are within the ball of radius $r$ defined above, they stay in the ball.

Note that by invoking Barbalat's lemma [44], it can be seen that the unforced system $\left(f_{\text {ext }}^{0}=0\right.$ and $\left.\dot{k}=0\right)$ is globally asymptotically stable.

Remark 4.1 In the absence of external force, the control law (4.10) reduces to a position controller with variable proportional and derivative gains. Nevertheless, the control goal of an impedance controller is still different from that of a position controller.

\subsubsection{Force Controller}

The force controller is an outer-loop control that modifies the desired position trajectories of the impedance controller, see Fig. 4.5. Intuitively, the force controller's action can be considered as one that modifies the rest-length of the spring of the impedance controller given in (4.10). The force controller modifies the desired slave position command, $\bar{p}_{u}^{0}$ of the operator, as given in (4.9).

To achieve reasonable tracking performance of a time-varying reference force trajectory and zero steady state error, a proportional-integral control law is employed. If $\bar{F}_{\text {ext }}^{c}$ denotes the bounded user-defined force along the direction of interaction with bounded first derivative, the force control is given by

$$
m_{u} \Delta \ddot{p}_{u}^{0,0}+d(t) \Delta \dot{p}_{u}^{0,0}+k(t) \Delta p_{u}^{0}=\bar{f}_{e x t}^{0}-k_{p} e_{f}-k_{i} \int e_{f} d t
$$

where $e_{f}:=f_{\text {ext }}^{0}-\bar{f}_{\text {ext }}^{0}$, in which $\bar{f}_{\text {ext }}^{0}=R_{c}^{0}\left[\begin{array}{ll}0 & \bar{F}_{\text {ext }}^{c}\end{array}\right]^{T} ; k_{p}$ and $k_{i}$ denote the proportional and integral control gains. The contact detection and contact point identification that are required for the application of the above force control law are given in the next section.

The force controlled aerial robot dynamics can be rewritten in differential form by combining (4.8), (4.9) and (4.16), i.e.,

$$
m_{u}\left(\dddot{p}_{u}^{0,0}-\dddot{\ddot{p}}_{u}^{0,0}\right)+d(t)\left(\ddot{p}_{u}^{0,0}-\ddot{\bar{p}}_{u}^{0,0}\right)+k(t)\left(\dot{p}_{u}^{0,0}-\dot{\bar{p}}_{u}^{0,0}\right)=-\tilde{k}_{p} \dot{e}_{f}-k_{i} e_{f}
$$

where $\tilde{k}_{p}=1+k_{p}$.

Proposition 4.2 A properly tuned force control law (4.16) results in ISS system with inputs $\dot{\bar{f}}_{\text {ext }}^{0}$ and $\bar{f}_{\text {ext }}^{0}$. 
Proof: Without loss of generality, consider interaction with a vertical wall, a constant stiffness and damping of the impedance controller, $x_{e}^{0}=0$, and $\bar{p}_{u}^{0}=0$. Following Assumption 3, (4.8), (4.9) and (4.16), the closed loop dynamics of the force controlled DOF can be rewritten as

$$
\begin{aligned}
& m_{u} \dddot{p}_{u}^{0,0}[1]+d \ddot{p}_{u}^{0,0}[1]+\left(k+\tilde{k}_{p} \tilde{k}_{e}\right) \dot{p}_{u}^{0,0}[1]+k_{i} \tilde{k}_{e} p_{u}^{0}[1] \\
& =\tilde{k}_{p} \ddot{\bar{f}}_{\text {ext }}^{0}[1]+k_{i} \bar{f}_{\text {ext }}^{0}[1]
\end{aligned}
$$

where $\tilde{k}_{e} \approx k_{e}{ }^{3}$

Clearly, (4.18) can be represented by linear state space form of $\dot{x}=A x+B u$, with

$$
\begin{aligned}
& x^{T}=\left[p_{u}^{0}[1], \dot{p}_{u}^{0,0}[1], \ddot{p}_{u}^{0,0}[1]\right], \quad u^{T}=\left[\dot{\bar{f}}_{\text {ext }}^{0}[1], \bar{f}_{e x t}^{0}[1]\right] \\
& A=\left[\begin{array}{ccc}
0 & 1 & 0 \\
0 & 0 & 1 \\
-\frac{k_{i} \tilde{k}_{e}}{m_{u}} & -\frac{k+\tilde{k}_{p} \tilde{k}_{e}}{m_{u}} & -\frac{d}{m_{u}}
\end{array}\right], \quad B=\left[\begin{array}{cc}
0 & 0 \\
0 & 0 \\
\frac{\tilde{k}_{p}}{m_{u}} & \frac{k_{i}}{m_{u}}
\end{array}\right]
\end{aligned}
$$

Linear systems are ISS if the system matrix is Hurwitzian [1]. Given that $m_{u}, d, k \in \mathbb{R}^{+}$, the system matrix $A$ of the above system is Hurwitzian provided that

$$
k_{i}<\frac{d}{m_{u}}\left(\tilde{k}_{p}+\frac{k}{\tilde{k}_{e}}\right)
$$

To make the proposed force controller robust against wide range of environmental stiffness, the control parameters can be tuned in such a way that the force controlled aerial robot is stable independently of $\tilde{k}_{e}$, i.e.,

$$
k_{i}<\frac{d}{m_{u}} \tilde{k}_{p}
$$

Once the boundedness of the response from the desired force trajectory to the position of the aerial robot is ensured, the ISS of the force controlled dynamics can now be illustrated. If $v:=m_{u}\left(\dddot{p}_{u}^{0,0}-\dddot{\bar{p}}_{u}^{0,0}\right)+d\left(\ddot{p}_{u}^{0,0}-\ddot{\bar{p}}_{u}^{0,0}\right)+k\left(\dot{p}_{u}^{0,0}-\dot{\bar{p}}_{u}^{0,0}\right),(4.17)$ can be rewritten as

$$
\begin{aligned}
& \tilde{k}_{p} \dot{e}_{f}+k_{i} e_{f}=-v \\
\Leftrightarrow \quad & \dot{e}_{f}+\frac{k_{i}}{\tilde{k}_{p}} e_{f}=-\frac{1}{\tilde{k}_{p}} v
\end{aligned}
$$

Note that (4.21) is a first order differential equation driven by a decaying input $v$. As a result, the above system is ISS provided that $k_{p}, k_{i} \in \mathbb{R}^{+}$.

\subsubsection{Sliding-mode Observer}

The estimation of the interaction force is formulated as state and disturbance estimation problem, by means of a second order sliding-mode observer. The main idea behind sliding-mode observers is the injection of a discontinuous term, which

\footnotetext{
3 To be more precise, $\tilde{k}_{e}=k_{e}+k_{g}$, where $k_{g}$ represents the effect of the change in geometry resulting from the differential Jacobian [11], and coupling with the rotational dynamics. Since $k_{g}$ is not significant, it is disregarded. Note that disregarding $k_{g}$ does not affect the stability analysis as it can be consider as a bounded exogenous perturbation.
} 
depends on the output, in order to force the error dynamics to remain on a sliding surface [115].

A cascaded sliding-mode observer is considered for the states and external force estimation. ${ }^{4}$ If a fast inner-loop observer for the rotational dynamics is assumed, the state space representation of the dynamics of the aerial robot used for observation can be given as a second order decoupled dynamics along each DOF, as follows:

$$
\left\{\begin{array}{l}
\dot{x}_{1}^{i}=x_{2} \\
\dot{x}_{2}^{i}=-\frac{d^{i}}{m^{i}} x_{2}+\frac{1}{m^{i}} u^{i}+\frac{1}{m^{i}} v^{i} \\
y^{i}=x_{1}^{i}
\end{array}\right.
$$

where the superscript $i \in\{x, y, \theta\}$ denotes the two translational DOFs along $x$ and $y$ axes, and the rotational DOF; $x=\left[\begin{array}{cc}x_{1}^{i} & x_{2}^{i}\end{array}\right]^{T}$ are the state variables, in which $x \in\left\{x_{u}^{0}, y_{u}^{0}, \theta\right\} ; d^{i} \in\left\{d_{x}, d_{y}, d_{\omega}\right\} ; m^{i} \in\left\{m_{u}, J_{u}\right\}$; by abuse of notation, $u^{i}$ collects the control input and other state independent forces such as gravity; $v^{i}$ is a bounded disturbance due to interaction, i.e., $\left\|v^{i}\right\| \leqslant \rho^{i}<\infty$. $\rho^{i}$ is a constant, representing the estimated maximum value of the disturbance along axis $i$.

Note that the dynamics of each DOF of the aerial robot, as given in (4.22), is observable. Therefore, there exists a Leuenberger observer with gain $L^{T}=$ $\left[\begin{array}{ll}L_{1} & L_{2}\end{array}\right]$ such that the eigenvalues of $(A-L C)$ are in the open left half of the complex plane. Besides the linear observer, a second order sliding-mode term that depends on the output is injected to the designed observer to ensure robust estimation of the states. The injected discontinuous term is further used to estimate the interaction force.

Inspired by [76], the designed observer is given by,

$$
\left\{\begin{array}{l}
\dot{\hat{x}}_{1}^{i}=\hat{x}_{2}^{i}-L_{1} e_{1}^{i}-k_{1}\left|e_{1}^{i}\right|^{1 / 2} \operatorname{sign}\left(e_{1}^{i}\right) \\
\dot{\hat{x}}_{2}^{i}=-\frac{d^{i}}{m^{i}} \hat{x}_{2}^{i}-L_{2} e_{1}^{i}-k_{2} \operatorname{sign}\left(e_{1}^{i}\right)+\frac{1}{m^{i}} u^{i}
\end{array}\right.
$$

where $e_{1}^{i}:=\hat{x}_{1}^{i}-x_{1}^{i}$ and $e_{2}^{i}=\dot{e}_{1}^{i}$ and

$$
\operatorname{sign}(a)= \begin{cases}\frac{a}{\|a\|} & \text { for } a \neq 0 \\ 0 & \text { for } a=0\end{cases}
$$

From (4.22) and (4.23), the estimation error dynamics is

$$
\left\{\begin{array}{l}
\dot{e}_{1}^{i}=e_{2}^{i}-L_{1} e_{1}^{i}-k_{1}\left|e_{1}^{i}\right|^{1 / 2} \operatorname{sign}\left(e_{1}^{i}\right) \\
\dot{e}_{2}^{i}=-\frac{d^{i}}{m^{i}} e_{2}^{i}-L_{2} e_{1}^{i}-k_{2} \operatorname{sign}\left(e_{1}^{i}\right)-\frac{1}{m^{i}} v^{i}
\end{array}\right.
$$

Proposition 4.3 A properly tuned observer given in (4.23) asymptotically estimates the states of the aerial robot in finite time $T$, even in the presence of bounded disturbance (primarily from the interaction force) [See the Appendix $C$ for the proof].

\footnotetext{
${ }^{4}$ Cascaded sliding-mode observer is chosen only for its simplicity. A nonlinear sliding-mode observer can also be designed for the complete systems without the cascaded structure as [76]. Such choice does not really hinder the development of the proposed control architecture.
} 
Based on the above proposition, for $t>T, e_{1}^{i} \rightarrow 0 \Rightarrow \hat{x}_{1}^{i} \rightarrow x_{1}^{i}$ and $e_{2}^{i} \rightarrow 0 \Rightarrow$ $\hat{x}_{2}^{i} \rightarrow x_{2}^{i}$. Thus, the error dynamics can be approximated as

$$
\left\{\begin{array}{l}
\dot{e}_{1}^{i} \approx 0 \\
\dot{e}_{2}^{i} \approx-k_{2} \operatorname{sign}\left(e_{1}^{i}\right)-\frac{1}{m^{i}} v^{i} \approx 0
\end{array}\right.
$$

Once the system's state trajectories reach the sliding surface, the discontinuous output injection signal relation obtained in (4.26) approximates the disturbance along axis $i$, i.e.,

$$
\hat{v}^{i}=-m^{i} k_{2} \operatorname{sign}\left(e_{1}^{i}\right)
$$

\subsubsection{Force Estimation}

In the literature, various methods have been proposed to recover the continuous time signal form of the unknown disturbance from the switching signal. The methods include low-pass filtering [6], nonlinear saturated function, boundary layering [116]. Here, the disturbance is estimated by low-pass filtering and thresholding $\hat{v}^{i}$, i.e.,

$$
\dot{\tilde{v}}^{i}=\frac{\hat{v}^{i}-\tilde{v}^{i}}{\tau_{f}}
$$

where $\tau_{f}$ is the time constant of the first order low-pass filter, and $\tilde{v}^{i}$ is the filtered version of $\hat{v}^{i}$. $\tilde{v}^{i}$ is further modified as follows.

$$
\bar{v}^{i}= \begin{cases}\tilde{v}^{i} & \text { for }\left|\tilde{v}^{i}\right|>\delta \\ 0 & \text { Otherwise }\end{cases}
$$

where $\delta$ is a small positive number that increases robustness against false contact detection. $\bar{v}^{i}$ is in fact the estimate of the interaction force.

$$
\begin{aligned}
& \hat{f}_{\text {ext }}^{0}=\left[\begin{array}{ll}
\bar{v}^{x} & \bar{v}^{y}
\end{array}\right]^{T} \\
& \hat{\tau}_{\text {ext }}^{u}=\bar{v}^{\theta}
\end{aligned}
$$

\subsubsection{Contact Detection and Contact Point Identification}

Contact is detected based on the magnitude of the estimates of the interaction force. Let $s$ be a binary that represents the contact detection, such that

$$
s= \begin{cases}1 & \text { if }\left\|\hat{f}_{\text {ext }}^{0}\right\|>\sigma \\ 0 & \text { Otherwise }\end{cases}
$$

where $\sigma$ is a positive constant that denotes the threshold beyond which contact is detected.

Once contact is detected, the point of contact needs to be identified to apply the force control law. The contact point can be uniquely defined by $\left(p_{c}^{u}, R_{c}^{0}\right)$ pairs representing its displacement from the $\mathrm{COM}$ of the aerial robot and its orientation 


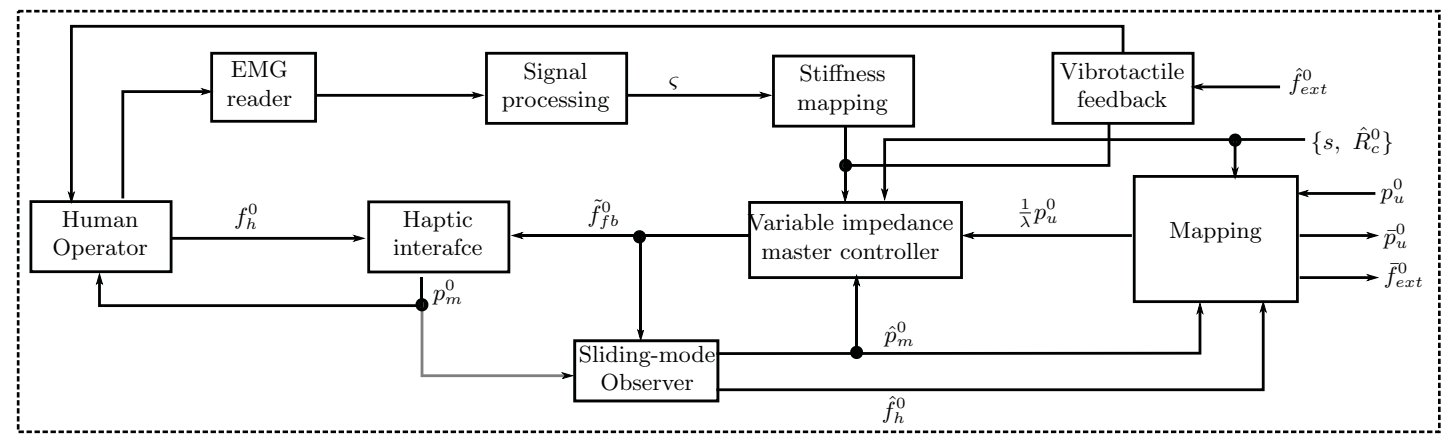

Figure 4.6: Block diagram representation of the master control architecture, which is responsible for mapping the operator's command and displaying multimodal feedbacks to restore a sense of direct interaction. The gray signals indicate measurement data.

with respect to $\psi^{0} . R_{c}^{0}$ is estimated based on the external force estimate given by (4.30), i.e.,

$$
\hat{R}_{c}^{0}=\left[\begin{array}{cc}
\frac{\hat{f}_{e x t}^{0}[2]}{\left\|\hat{f}_{e x t}^{0}\right\|} & \frac{\hat{f}_{e x t}^{0}[1]}{\left\|\hat{f}_{x x t}^{0}\right\|} \\
-\frac{\hat{f}_{e x t}^{0}[1]}{\left\|\hat{f}_{e x t}^{0}\right\|} & \frac{\hat{f}_{e x t}^{0}[2]}{\left\|\hat{f}_{e x t}^{0}\right\|}
\end{array}\right]
$$

On the other hand, based on Assumption 2, only the $y$-axis coordinate of $p_{c}^{u}$, i.e., $p_{c}^{u}[2]$, needs to be determined. Its estimate is given by

$$
p_{c}^{u}[2]= \begin{cases}\frac{p_{c}^{u}[1] \hat{f}_{e x t}^{u}[2]-\hat{\tau}_{e x t}^{u}}{\hat{f}_{\text {ext }}^{u}[1]} & \text { if }\left|\hat{f}_{\text {ext }}^{u}[1]\right|>\varrho \\ 0 & \text { otherwise }\end{cases}
$$

where $\varrho \in \mathbb{R}^{+}$is a small number introduced to avoid numerical singularity, and $\hat{f}_{\text {ext }}^{u}=R_{0}^{u} \hat{f}_{\text {ext }}^{0}$.

The estimated configuration of the contact point can also be used, among others, to

- make sure the detection is within the physical boundary of the slave aerial robot.

- construct the Jacobian ( $J$ mentioned in Section 4.3.2) of the contact point if one needs to modify the apparent impedance as seen from the contact point.

\subsection{The Master Control Architecture and Multi- Modal Feedback}

This section discusses the master controller, which is responsible for detecting the intention of the operator and displaying feedback to create situational awareness of the controlled slave and/or its environment.

Master-slave workspace incompatibility is one of the main issues in haptic teleoperation of aerial robot [67]. However, to focus on the main contributions of this work, without loss of generality, a position to position mapping with a mapping 
scale $\lambda$ is considered in this work, i.e., $\bar{p}_{u}^{0,0}=\lambda p_{m}^{0,0}$. Interested readers in mapping strategies are referred to [36], [84], [53], [68] and Chapter 2.

Fig. 4.6 shows the proposed control architecture of the master system. The main points addressed in the subsequent sections are the stiffness mapping, force feedback and vibrotactile feedback.

\subsubsection{Stiffness Mapping}

For stiffness command for the variable impedance controller, an inspiration is drawn from the human arm. Humans change their arm impedance by changing their muscle length through sophisticated neuromuscular activities. These activities can be observed through EMG signals. Although detailed analysis on derivation of stiffness command from EMG signals is a separate research topic and is beyond the scope of this work, a proper motivation and a rough sketch on how it is incorporated in the proposed control architecture is provided.

Estimation of the human arm stiffness based on a second order dynamic model of the arm and EMG signals has been proposed in the literature, [104], [75]. Recently, the use of compound command of human arm position and stiffness has been proposed for unilateral telemanipulation [2]. Similarly, impedance command based on grip force have been used in open-loop control of the impedance of a 1D slave robot, [117].

EMG signals acquired from muscular contraction have also been widely used for control of hand prosthesis [52]. They are preferred over other signals because they are stable, adaptable and easily acquired using noninvasive methodologies. Besides, the aforementioned characteristics, the use of EMG signals as stiffness command is motivated by recent studies that indicated approximated linear dependency of force/stiffness to muscular activation/co-activation [104].

Although the operator's hand (point of interaction with the haptic interface) stiffness is a function of the arm movement and EMG signals, by assuming slow arm movement, the stiffness command for the slave controller is derived solely from EMG signals, acquired from upper arm muscular movements of the operator by using electrodes. After EMG acquisition, the signals are processed as follows,

- High-pass filtration: to remove low frequency signals stemming from motion artifacts and instability of the skin-electrode interface.

- Rectification: to generate unidirectional signal.

- Low-pass filtration: to remove high frequency noise and irrelevant details.

- Mapping: to map the processed EMG signal to appropriate stiffness command. The chosen mapping function is given by

$$
\bar{k}(t)=\frac{\bar{k}_{\max }-\bar{k}_{\min }}{\varsigma_{\max }-\varsigma_{\min }} \varsigma(t)+\bar{k}_{\min }
$$




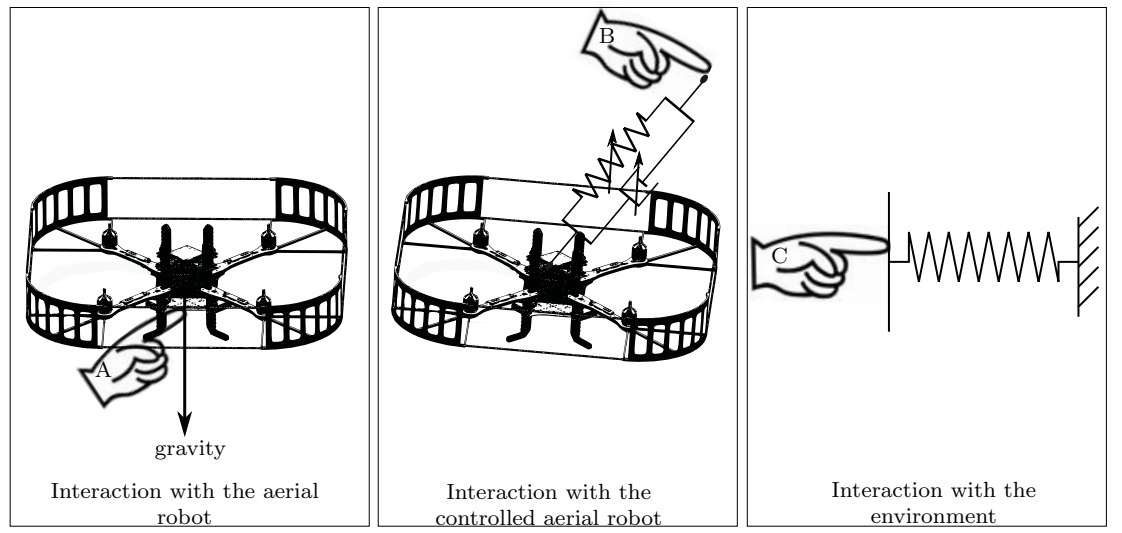

Figure 4.7: Exemplary alternatives of direct interactions that can be emulated by the teleoperator during free-flight and interaction.

where $\varsigma$ denotes the strength of the processed EMG signal, in which $\varsigma_{\min }=0$; $\bar{k} \in \mathbb{R}^{+}$is the stiffness command.

\subsubsection{Force Feedback}

In haptic teleoperation, the force feedback is primarily aimed at restoring the sense of direct interaction of the operator with the manipulated object, usually the environment. In haptic teleoperation of aerial robots, however, the flight regime includes a considerable free-flight, in which, the manipulated object of interest is the slave aerial robot. Therefore, depending on the flight regime, the proposed force feedback attempts to establish direct interaction with the aerial robot and/or the environment.

The first two illustrations in Fig. 4.7 show alternatives by which direct interaction can be emulated during free-flight. The first attempts to create direct maneuvering of the aerial robots by the operator, where the operator is also responsible to compensate for the action of gravity, friction and other undesired dynamics. However, such situation quickly exhausts the operator during the teleoperation of the aerial robot. Instead, the second alternative attempts to establish the operator's interaction with the impedance controlled aerial robot, where unwanted forces and higher dynamics are already hidden by the action of the slave controller. In this case, the force feedback during free-flight, $f_{f f}^{0}$, is equivalent to the force required to maneuver the aerial robot that flies in gravityless and frictionless environment. It is given by

$$
f_{f f}^{0}=\frac{1}{\lambda}\left\{-m_{u} \ddot{\bar{p}}_{u}^{0,0}+d(t) \dot{e}_{p}+k(t) e_{p}\right\}
$$

The third alternative in Fig. 4.7 attempts to establish a direct interaction with the manipulated environment during interaction. In this scenario, the force feedback $f_{f i}^{0}$ is the scaled version of the estimated external force given by (4.30), i.e.,

$$
f_{f i}^{0}=-\frac{1}{\lambda} \hat{f}_{e x t}^{0}
$$


Since the aerial robot has multiple DOFs, simple switching between the above two forces depending on the flight regime does not capture all possible scenarios along the different DOFs. For instance, the aerial robot can interact with an environment along the $x$ - axis while sliding along the $y$ - axis. Therefore, once contact is detected, the component of $f_{f f}^{0}$ along the axis of interaction (i.e., $y$ - axis of $\psi^{c}$, see also Assumption 4.2) is replaced by the interaction force. In this manner, a sense of direct interaction with the environment and the aerial robot, along the axis of interaction and other axis orthogonal to it is established, respectively. Mathematically, the force feedback $f_{f b}^{0}$ displayed to the operator is given by

$$
f_{f b}^{0}=f\left(f_{f f}^{0}, f_{f i}^{0}\right)= \begin{cases}f_{f f}^{0} & \text { when } s=0 \\
\hat{R}_{c}^{0}\left[\begin{array}{l}
\left.f_{f f}^{c}[1]\right] \\
f_{f i}^{c}[2]
\end{array}\right] & \text { othewise }\end{cases}
$$

where $s$ is as given in (4.31); $f_{f f}^{c}=\hat{R}_{0}^{c} f_{f f}^{0}$ and $f_{f i}^{c}=\hat{R}_{0}^{c} f_{f i}^{0}$.

The force feedback given in (4.37) guarantees the operator to intuitively interact with the aerial robot and/or the environment, depending on contact. However, it requires switching between (4.35) and (4.36) along the axis transiting from freeflight to interaction or vice versa. To avoid switching, it is proposed to display a modified force feedback generated by a variable impedance master controller.

In fact, $f_{f f}^{0}$ in (4.35) can be achieved by rendering the mass of the controlled haptic device (given in (4.7)) as $m_{m}=m_{u}$, and using the same spring and damper as in the impedance controller of the slave. But this spring and damper act on the scaled version of $e_{p}$ and $\dot{e}_{p}$, i.e., $\frac{1}{\lambda} e_{p}$ and $\frac{1}{\lambda} \dot{e}_{p}$. The master controller also includes an additional variable spring and damper, $k(t, s)$ and $d(t, s)$, which are modulated by contact. $k(t, s)$ acts on the deviation of the position of the master from its slave equivalent, i.e., $\tilde{e}_{p}=\left(p_{u}^{0,0}-\bar{p}_{u}^{0,0}\right)$. When contact is detected, the spring constant along the axis of the contact is smoothly increased from zero to a large value that can be rendered by the haptic interface, i.e., $k(t, s) \in\left[0, k_{\max }\right]$. The damper $d(t, s)$ is changed accordingly to achieve critical damped response. As such, a correspondence between the position of the master and the slave can be established, even during interaction, while tracking a desired interaction force. The adaptation laws of $k(t, s)$ and $d(t, s)$ are similar to (4.12) and (4.13).

The modified force feedback $\tilde{f}_{f b}^{0}$ is given by

$\tilde{f}_{f b}^{0}=\frac{1}{\lambda} \hat{R}_{c}^{0}\left[\begin{array}{c}m_{u} \ddot{\bar{p}}_{u}^{c, 0}[1]+d(t) \dot{e}_{p}^{c}[1]+k(t) e_{p}^{c}[1] \\ m_{u} \ddot{\bar{p}}_{u}^{c, 0}[2]+d(t) \dot{e}_{p}^{c}[2]+k(t) e_{p}^{c}[2]+d(t, s) \dot{\tilde{e}}_{p}^{c}[2]+k(t, s) \tilde{e}_{p}^{c}[2]\end{array}\right]$

where $e_{p}^{c}$ is the position error of the aerial robot expressed in coordinates of $\psi^{c}$.

Proposition 4.4 The controlled dynamics of the haptic interface (4.7) with haptic feedback (4.38) is ISS with input $f_{h}^{0}, \dot{p}_{u}^{0,0}, p_{u}^{0}, \Delta \dot{p}_{u}^{0,0}$, and $\Delta p_{u}^{0}$.

Proof: $\quad$ The proof is similar to the proof of Proposition 4.1.

Remark 4.2 The force feedback during free-flight, given by (4.35), is just one type and can be different depending on the employed impedance controller. 
Remark 4.3 When displaying $\tilde{f}_{f b}^{0}$ in (4.38), there is a slight deviation from ideal feeling of direct interaction during transients from free-flight to contact. However, in low velocity flights, the inertial forces responsible for the deviation are negligible. Alternatively, in contact phase, the apparent inertia of the aerial robot along the axis of interaction can be modified by control action to a negligible amount. $\triangleleft$

\subsubsection{Vibrotactile Feeback}

The use of vibrotacticle feedback is proposed to supplement the haptic feedback, with an ultimate goal of improving the operator's awareness of the manipulated object. Here, the vibrotactile feedback is used to close the stiffness control loop, which, otherwise, is open [2]. In the presented control architecture, the use of two sets of motors that are capable of providing tactile feedback with varying frequencies are proposed. The first set provides cue about the stiffness of the impedance controller. The second set, on the other hand, is used for discrimination of environmental stiffness. The environmental stiffness is estimated based on the estimate of the interaction force (4.32) and Assumption 4.3. A linear relation between the stiffness of the manipulated object and frequency of vibration is used. Note that the activation of the second set of motors also indicates the occurrence of contact.

\subsubsection{Operator Force Estimation}

The force command for the slave's force controller is derived from the operator. This force is estimated using a sliding-mode observer, similar to the one presented in Section 4.3.3. Unlike the slave aerial robot, the most common point of interaction between the operator and the master is a single point and is often the tip of the haptic interface. Even if a force sensor can also be employed, an observer is used to maintain the generality of the proposed control architecture. Moreover, such estimation of the operator's force can also be used for secondary tasks, when the goal is not even force tracking.

\subsection{Practical Considerations}

For effective utilization of the proposed tele-control architecture, besides the practical considerations provided in the previous chapters, certain additional considerations are recommended, of which some of them are described here.

Tuning Force Controller Parameters: Since the proposed force controller is impedancebased (indirect/implicit force control), its bandwidth should be less than the bandwidth of the inner-loop variable impedance controller. Therefore, the force control parameters, $k_{p}$ and $k_{i}$, should be selected by taking this into account.

Moreover, to limit unnecessary buildup, the integral action of the force controller is replaced by a nonlinear saturated integral action that is reset when there in no interaction. 


$$
\xi= \begin{cases}k_{i} \int e_{f} d t & \text { when } s=1 \text { and }|\xi|<\xi_{\max } \\ \xi_{\max } & \text { when } s=1 \text { and }|\xi|>\xi_{\max } \\ 0 & \text { otherwise }\end{cases}
$$

where $\xi_{\max }$ is the saturation limit of the integral action.

Smooth Trajectory Generation: In general, the inner-loop variable impedance controller intrinsically handles the transition between interaction and free-flight of the aerial robot in a smooth manner. Moreover, the reference trajectory generated to track the desired interaction force is smooth as well. Nevertheless, in transiting from contact to free-flight, disengaging the force control loop quickly results in rapid change in the reference trajectory. To avoid such situations, a $5^{\text {th }}$ order polynomial is used to generate a smooth trajectory that fulfills both the initial and final acceleration, velocity and position constraints. The final value constraints are

$$
\Delta \ddot{p}_{u}^{0,0}\left(t_{0}+\delta t\right)=\Delta \dot{p}_{u}^{0,0}\left(t_{0}+\delta t\right)=\Delta p_{u}^{0}\left(t_{0}+\delta t\right)=0
$$

where $t_{0}$ denotes the time at which the trasition from contact to free-flight starts, and $\delta t$ denotes a priori set time-interval, within which, the force controller fully disengages.

Sign function: Similar to [116], the sign(·) function can be replaced by a saturation function

$$
\operatorname{sat}(\mathrm{a}, \epsilon)= \begin{cases}\frac{a}{\|a\|} & \text { for }\|a\|>\epsilon \\ \frac{a}{\epsilon} & \text { for }\|a\| \leq \epsilon\end{cases}
$$

where $\epsilon$ is a threshold, below which the $\operatorname{sign}(\cdot)$ function is approximated.

Such approximation of the $\operatorname{sign}(\cdot)$ function minimizes the switching, which is computationally demanding. However, the error dynamics becomes bounded rather than converging to zero.

The Force Estimator: Properly selecting the time constant of the low-pass filter in Section 4.3.3 is enough to reasonably reconstruct the external force $F_{\text {ext }}$ without introducing significant delay. However, in the presence of low-frequency noise, additional filters can replace or be used in combination with the low-pass filter.

$\epsilon$ in (4.41) can also be selected depending on the magnitude of the noise signal. As such, the chattering that can be caused by the sign $(\cdot)$, mainly due to the noise signal, can be greatly reduced. Note that, however, the smaller $\epsilon$, the better the state estimation becomes. Hence, there is a trade-off between the achievable estimation accuracy and reduction of chattering.

Thrust approximation: The thrust generated by the propeller/s of the aerial robot are required by the sliding-mode observer algorithm, see (4.22). However, direct measurement of the thrust is often not available. Nonetheless, it can be approximated by off-line identification, similar to [63], [7].

Vibrotactile Feedback: The range of frequencies used for tactile feedback should, in general, fall within the optimal frequency range to activate the mechanoreceptors of the skin.

It is also recommended to use more than one motor in each set of the tactile feedback device, and alternate among them while generating the feedback. This 
is to avoid unconscious filtration of the feedback, as the mechanoreceptors become less sensitive after a prolonged stimulation at the same place.

Scaling Force Feedback: Due to the workspace incompatibility between the master and the slave, master motion is scaled to the slave motion at the expense of the force feedback reduction. Hence, significant scaling down of the force feedback may lead to degradation of sensitivity of the slave's motion or the interaction force. To mitigate such problems, the force feedback $\tilde{f}_{f b}^{0}$ can be further scaled depending on the operator's convenience.

\subsection{Simulations}

Several simulations have been run in the software package 20Sim (Controllab Products B.V., Enschede, The Netherlands) and representative results are presented in this section. A bond graph model of a planar ducted-fan aerial robot is used as the slave aerial robot [66], [80]. The model also includes aerodynamic effects and uncertainties that are disregarded during controller design. The operator is modeled as a variable PD controller on the desired position of the master. Without loss of generality, the scaling factor $\lambda=1$ is used in all simulations. All the nominal control and model parameters used in the simulations are given in Table 4.1.

Table 4.1: Parameters used in simulation \{in SI units $\}$

\begin{tabular}{|c|c|}
\hline Operator's PD parameters & Environmental stiffness \\
\hline$k_{m} \in[1,14], d_{m} \in[2.24,8.37]$ & $k_{e}=1000$ \\
\hline \multicolumn{2}{|c|}{ Ducted-fan parameters } \\
\hline$m_{u}=1.25, J_{u}=0.015$ & $d_{x}=d_{y}=1, d_{\omega}=0.1$ \\
\hline Impedance controller & Force controller \\
\hline$k \in[1,14], d \in[2.24,8.37]$ & $k_{p}=3, k_{i}=4$ \\
\hline \multicolumn{2}{|c|}{ Sliding-mode observer } \\
\hline$k_{1}=2, k_{2}=90$ & $L_{1}=3, L_{2}=60$ \\
\hline$\tau_{f}=0.1, \delta=0.15, \sigma=0.25$ & $\varrho=0.1, \rho^{x}=\rho^{y}=10, \rho^{\theta}=100$ \\
\hline
\end{tabular}

\subsubsection{Variable impedance in free-flight}

In this simulation, the performance of the proposed control algorithm is illustrated in a free-flight task. During the course of this simulation, a variable stiffness and a periodic trajectory are commanded by the operator. Moreover, disturbance forces, $f_{d}^{0}=-f_{\text {ext }}^{0}=[-3,0]^{T} \mathrm{~N}$ and $f_{d}^{0}=\left[-\frac{3 \sqrt{3}}{2},-1.5\right]^{T} \mathrm{~N}$ are applied on the aerial robot in the time intervals between $2.5-5.6 \mathrm{~s}$ and $12.5-15.6 \mathrm{~s}$ at $\mathrm{h}=0.1 \mathrm{~m}$ and $\mathrm{h}=-0.06 \mathrm{~m}$, respectively. While the observer is active, the force controller is not connected to the inner-loop impedance controller. As such, the different functionalities of the proposed controller are illustrated separately.

The results from this simulation are depicted in Fig. 4.8. In particular, Fig. 4.8a shows that the positions of the aerial robot (dashed-line) reasonably track 
the reference position trajectories (continuous-line) commanded by the operator. The pitch reference generated based on the lateral reference trajectory, due to the underactuation (see Section 4.3), is also shown in the figure. As can be seen from Fig. $4.8 \mathrm{~b}$, for similar motion profile, the tracking performance is improved when the impedance of the controller is higher (Fig. 4.8c), as theoretically expected. When the external forces are applied, only the translational dynamics along the direction of application is affected. The affected translational dynamics behave similar to a second order damped physical system, whereas the rotational dynamics attempts to reject the torque induced due to the external forces (Fig. 4.8a).

The force feedback displayed to the operator is shown in Fig. 4.8d. Observe that the force feedback is dominated by aerial robot's inertial forces during transients. Once the robots is in steady state, the force feedback is dominated by the potential force of the impedance controller, which is equivalent to the external force applied on the robot.

Fig. 4.8e shows the external forces applied to the aerial robot, and their estimation based on the algorithm provided in Section 4.3.3. Except a slight difference, the estimated force is similar to the actual force, illustrating the applicability of the estimation algorithm. A closer look at Fig. 4.8e shows the slight difference between the actual and estimated forces, which is due to the lags induced by the contact detection threshold and low-pass filter, see Section 4.3.3. Fig. 4.8f shows the time evolution of the identified point of external force application.

\subsubsection{Interaction force regulation}

In this simulation, force regulation along the $x$-axis, while maintaining constant altitude is considered. Fig. 4.9a shows the performance of the variable impedancebased interaction force tracking controller. Once contact is detected, the force applied by the operator is mapped as a command for the force controller. As detailed out in Section 4.3.2, the force controller modifies the virtual position of the impedance controller in order to track the desired time-varying interaction force (Fig. 4.9b). The proposed controller is able to track the desired interaction force command in the presence of time-varying impedance (Fig. 4.9c). Due to the underactuation of the aerial robot, time-varying interaction forces are tracked by changing the tilting angle of the robot (Fig. 4.9d). Fig. 4.9a also shows the force feedback displayed to the operator during the course of this simulation. It is worth noticing the position and the force correspondence between the human operator and the environment during interaction, and the position and the force correspondence between the human operator and the aerial robot during free-flight, thereby, the transparency of the proposed controller.

\subsubsection{Sliding on an inclined surface}

This simulation involves docking, undocking and interaction force tracking while sliding on a surface inclined at an angle of $15 \mathrm{deg}$ from the vertical axis. As can be seen in Figs. 4.10a and 4.10b, the lateral and altitude trajectories are well tracked. When the aerial robot docks on the surface $t \approx 6 \mathrm{~s}$, the force controller kicks in and 


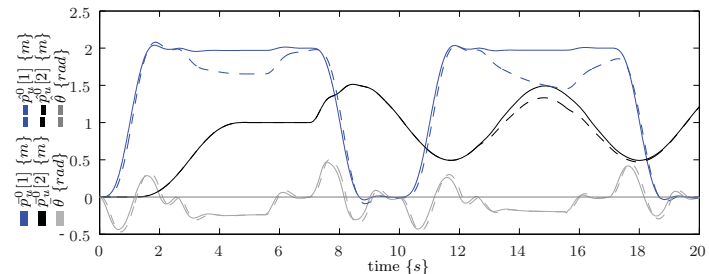

(a) The reference and actual slave trajectories.

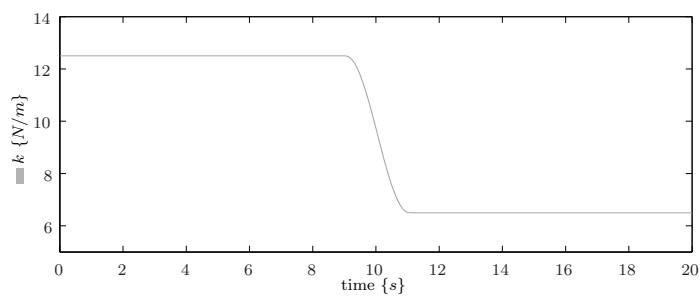

(c) The stiffness of the impedance controller.

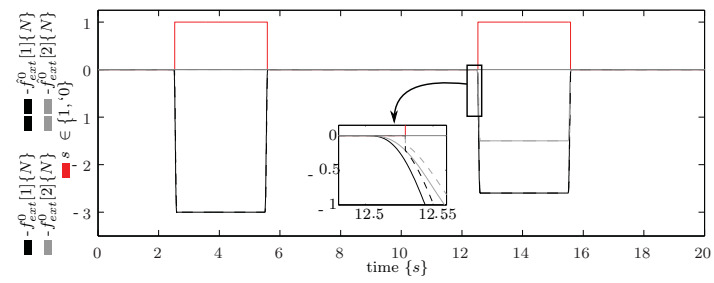

(e) External forces and their estimation. $s$ is the binary that indicates contact detection.

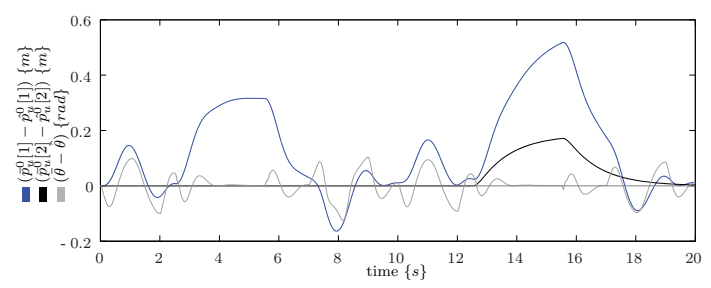

(b) Tracking error.

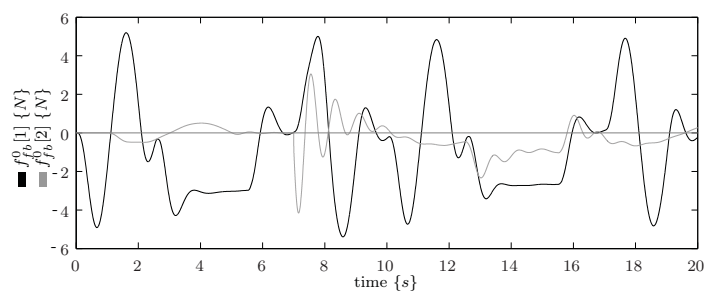

(d) Force Feedback

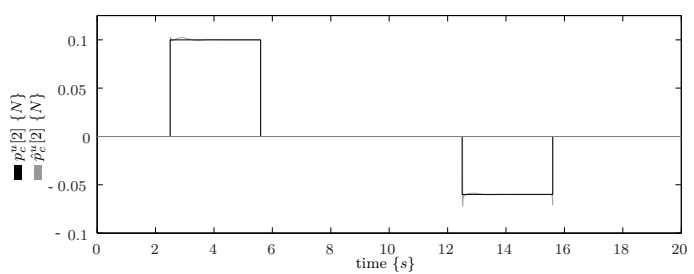

(f) Actual and identified point of external force application.

Figure 4.8: Results the variable impedance in free-flight simulation (in teleoperation mode).

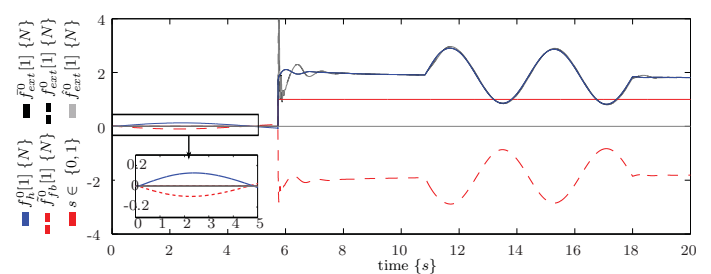

(a) The operator force, the force feedback displayed to the operator, the reference force, and the actual interaction force trajectories. After contact is detected $\bar{f}_{e x t}^{0}=f_{h}^{0}$.

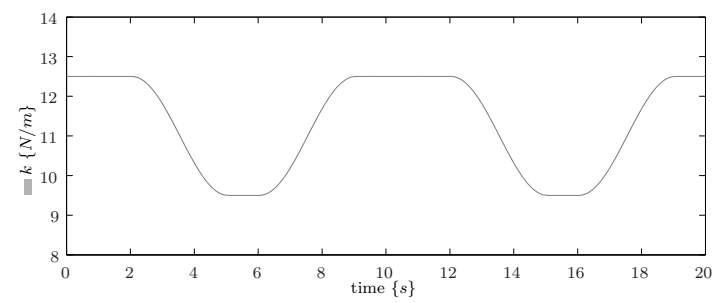

(c) Stiffness of the impedance controller

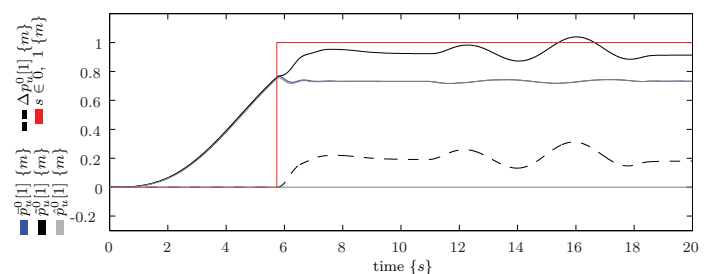

(b) Operator's position command, modified position due to the force controller, resultant position reference for the impedance controller, and actual slave position.

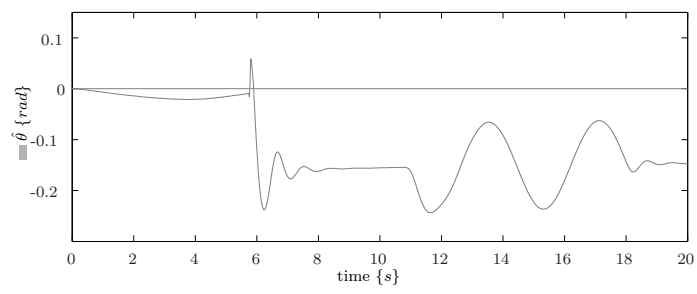

(d) pitch trajectory.

Figure 4.9: Results of the interaction force regulation simulation (in teleoperation mode). 


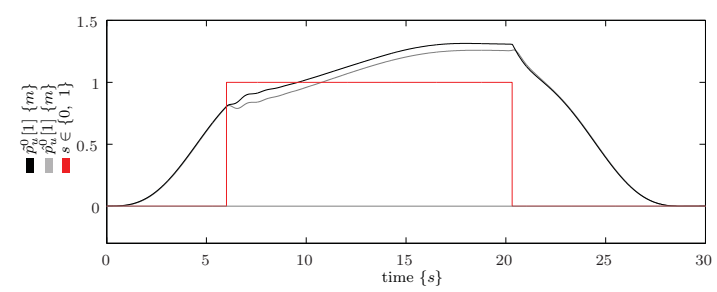

(a) The virtual position of the impedance controller and the position of the aerial robots along the lateral direction, both expresed in $\psi^{0}$.

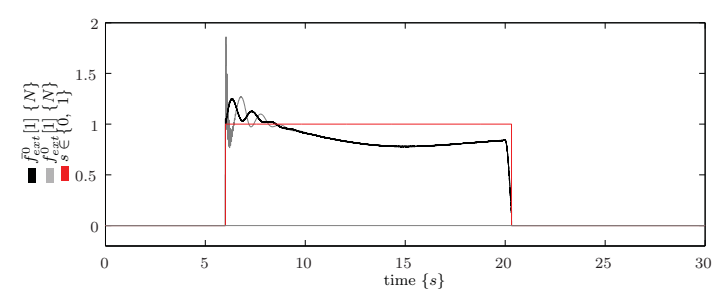

(c) The $x$ - component of the desired and actual interaction forces, both expresed in $\psi^{0}$.

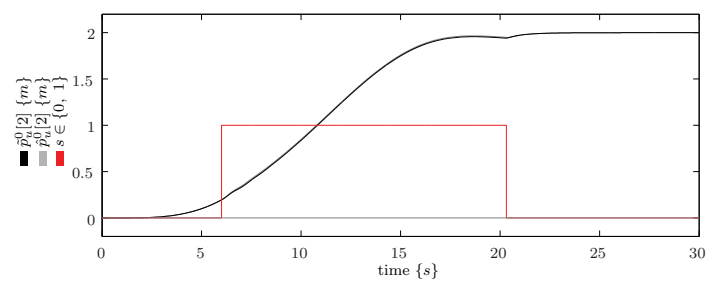

(b) The virtual position of the impedance controller and the position of the aerial robots along the vertical direction, both expresed in $\psi^{0}$.

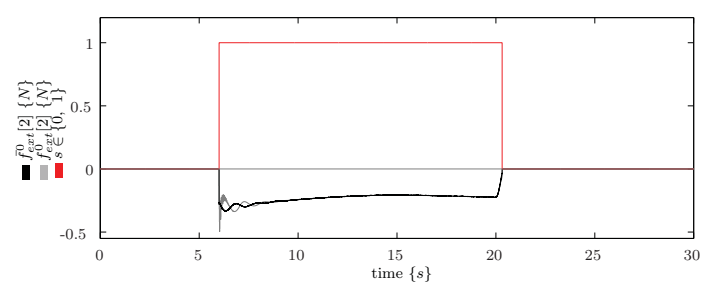

(d) The $y$ - component of the desired and actual interaction forces, both expresed in $\psi^{0}$.

Figure 4.10: Results of force regulation while sliding on inclined surface simulation (in teleoperation mode).

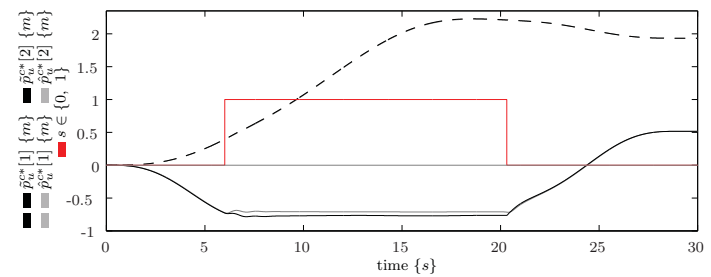

(a) The virtual position of the impedance controller and the position of the aerial robots, both expresed in $\psi^{c *}$.

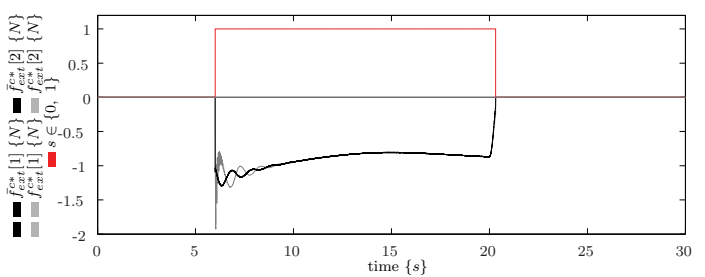

(b) The desired and actual interaction forces, both expresed in $\psi^{c *}$.

Figure 4.11: Positions and interaction force trajectories given in Fig. 4.10, expressed in $\psi^{c *}$, a coordinate frame which has the same origin as $\psi^{0}$, but the same orientation as $\psi^{c}$.

the desired interaction force is regulated along both $x$ and $y$ axis of $\psi^{0}$ (Figs. 4.10c and $4.10 \mathrm{~d})$. The interaction force perpendicular to the inclined surface is tracked, while simultaneously tracking a desired position along the inclined surface. For better intuition, the positions and the interaction forces, expressed in a coordinates of $\psi^{c *}$, whose origin and orientation are the same as $\psi^{0}$ and $\psi^{c}$, respectively, are shown in Figs. 4.11a and 4.11b. It is clear from these figures that, during contact, the motion along the $y-$ axis of $\psi^{c}$ is constrained, while it is free to slide along its $x-$ axis. At $t \approx 20.3 \mathrm{~s}$, the aerial robot undocks from the surface. 


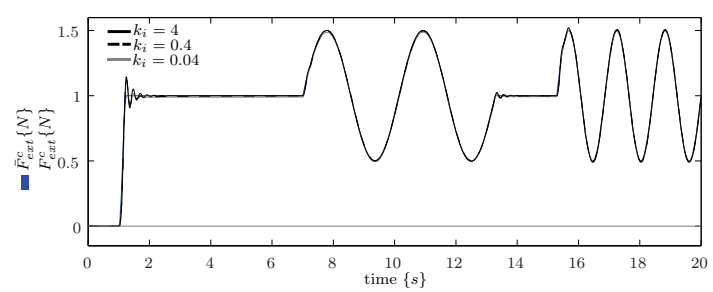

(a) Effect of varying $k_{i}$, and $k_{p}=0$ and $k_{e}=$ 1000.

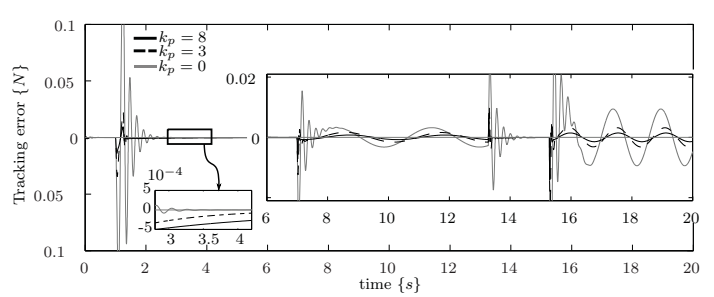

(c) Tracking error for varying $k_{p}$, when $k_{i}=4$ and $k_{e}=1000$.

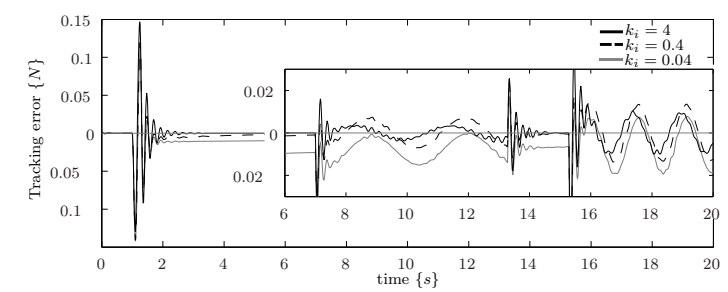

(b) Tracking error of varying $k_{i}$, when $k_{p}=0$ and $k_{e}=1000$.

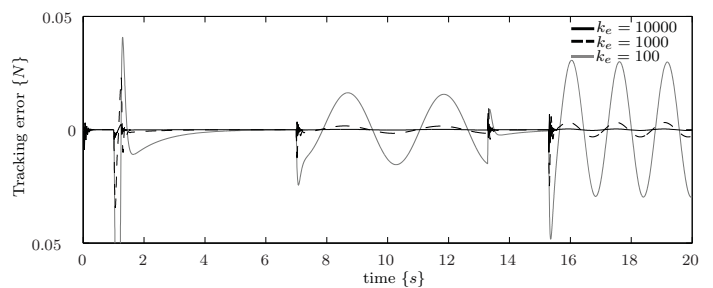

(d) Tracking error for varying $k_{e}$, when $k_{i}=4$ and $k_{p}=3$.

Figure 4.12: Results demonstrating the effects of $k_{p}, k_{i}$ and $k_{e}$ on the performance of interaction force regulation.

\subsubsection{Effects of $k_{p}, k_{i}$ and $k_{e}$ on force regulation performance}

Multiple simulations have been run to demonstrate the effects of the force controller parameters, $k_{p}$ and $k_{i}$, and the environmental stiffness, $k_{e}$, on the performance of force regulation. The desired force trajectory consists of a step reference of $1 \mathrm{~N}$ and periodic sine function with frequencies of $2 \mathrm{~Hz}$ and $4 \mathrm{~Hz}$, both with an amplitude of $0.5 \mathrm{~N}$.

Fig. 4.12a shows the effect of $k_{i}$ on the performance of the force tracking. Though it peaks higher for a step reference, the tracking error generally decreases and rapid convergence is achieved as $k_{i}$ increase (Fig. 4.12b). As it can easily be deduced from (4.21), this is a direct consequence of the increase in the bandwidth of the force controller as $k_{i}$ increases. Note also that for similar control parameters, the tracking error increases as the frequency of the reference increases.

Increasing $k_{p}$ appears to decrease the tracking error, see Fig. 4.12c. This is primarily due to the attenuation effect that $k_{p}$ has on the position tracking error, which acts as an input to the closed loop force controlled system, as described by (4.21). However, closer look at Fig. $4.12 \mathrm{c}$ reveals that increasing $k_{p}$, in fact, slows the convergence of the tracking error to zero. As expected from (4.21), increasing $k_{p}$ results in lower bandwidth, thereby, higher time constant. Therefore, from the above observations, increasing both $k_{i}$ and $k_{p}$ without saturating the actuator input and violating the stability constraint given in (4.20), provides higher tracking performance.

On the other hand, varying $k_{e}$ also affects the force tracking performance. Fig. $4.12 \mathrm{~d}$ shows results from different environments. The tracking performance deteriorates as the environment becomes more compliant. This is mainly due to 


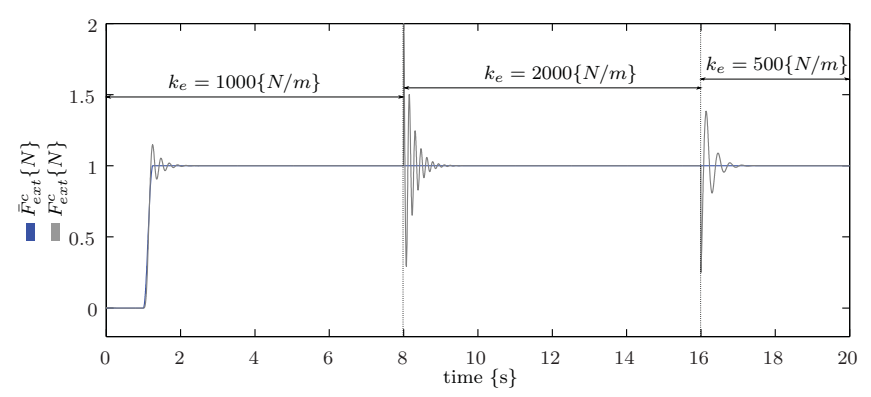

Figure 4.13: Performance of the force tracking controller during sudden change in environmental stiffness.

the bandwidth limiting effect that the environmental stiffness has on the innerloop impedance controller. During interaction, the resultant of the stiffness of the impedance controller and the environment significantly affects the bandwidth of the impedance controller. In general, lower resultant stiffness results in poor force tracking performance.

\subsubsection{Variable and nonlinear environmental stiffness}

Fig. 4.13 shows the performance of the force controller during sudden change of environment. As discussed in Section 4.3.2, the force controller is robust as long as its controller parameters are tuned to be robust against any linear environmental spring. Hence, the sudden changes in environmental stiffness to both lower and higher values results only in perturbation. Though the magnitude of perturbation depends on the change in environmental stiffness, the interaction force settles to the desired reference in due time.

Though the force controller is designed assuming environments that are characterized predominantly by linear spring (Assumption 4.3), its feasibility has been investigated for nonlinear environments as well. The environment is still assumed to be characterized by spring, whose dynamics is described by Hunt-Crossely contact model [38], i.e.,

$$
F_{e x t}^{0}= \begin{cases}k_{e}\left(\Delta x^{c}\right)^{n} & \text { for } \Delta x^{c}>0 \\ 0 & \text { otherwise }\end{cases}
$$

where $k_{e}$ denotes the contact stiffness, and $n$ is a constant real number, which is often between 1 and 2 .

It can be seen from Fig. 4.14 that the tracking performance in the case of nonlinear environment is less that its linear counterpart. The performance is better for $n$ close to 1 , higher values of $k_{e}$, and slowly varying references. Nevertheless, the tracking error remains bounded throughout. 


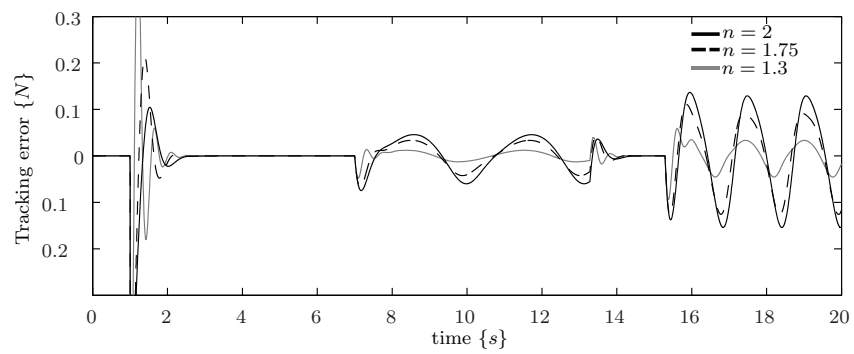

Figure 4.14: Performance of the force tracking controller during interaction with nonlinear environment.

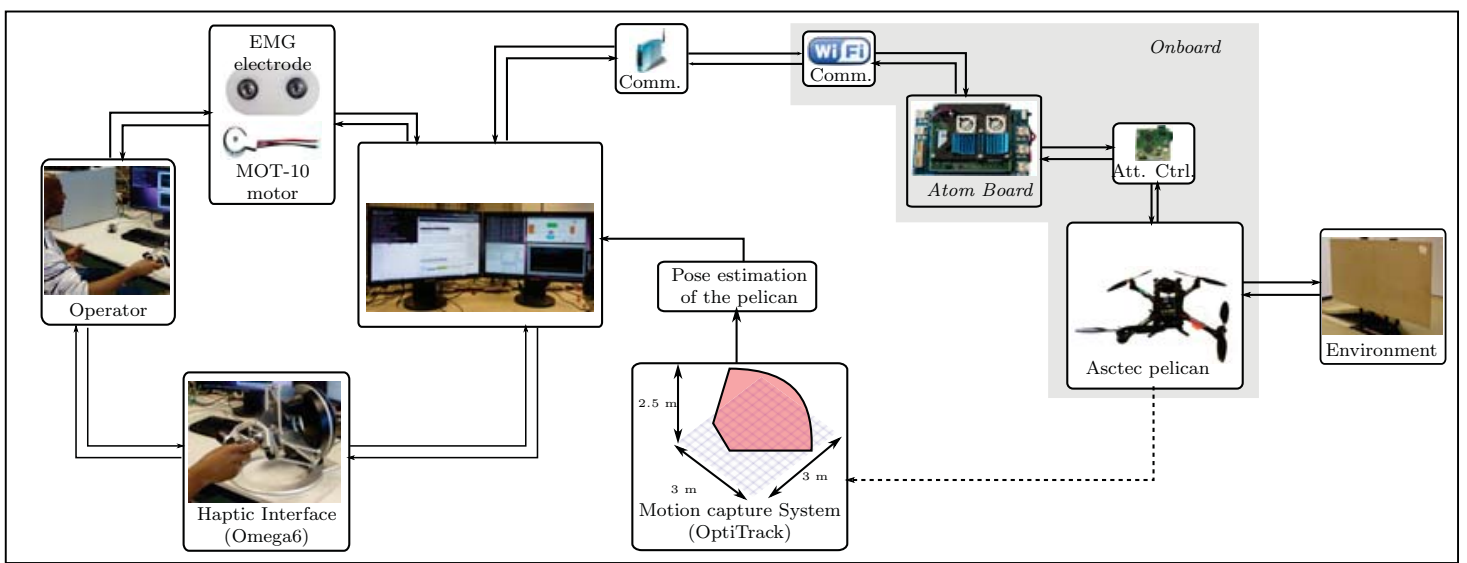

Figure 4.15: The complete hardware architecture.

\subsection{Experiments}

In this section, the experimental setup and results demonstrating the feasibility of the proposed control architecture are provided.

\subsubsection{Experimental Setup}

The implemented software architecture is based on ROS and MATLAB Simulink (MathWorks, Natick, MA, USA). Fig. 4.15 shows the hardware components used during practical realization of the proposed control architecture. The hardware architecture is similar to Chapter 2, but extended with additional components to realize the unique capabilities of the proposed controller. The human-machine interface is composed of ground PC, Omega6 (Force Dimension, Nyon, Switzerland) haptic interface, MOT-10 enclosed pager vibrator motor, and EMG electrodes. The slave robots is the Asctec Pelican quadrotor (Ascending Technologies GmbH, Krailling, Germany). The flight arena is of size $3 m \times 3 m \times 2.5 m$, and its equipped with Optitrack (NaturalPoint, Inc. Corvallis, OR, USA) external position system. 


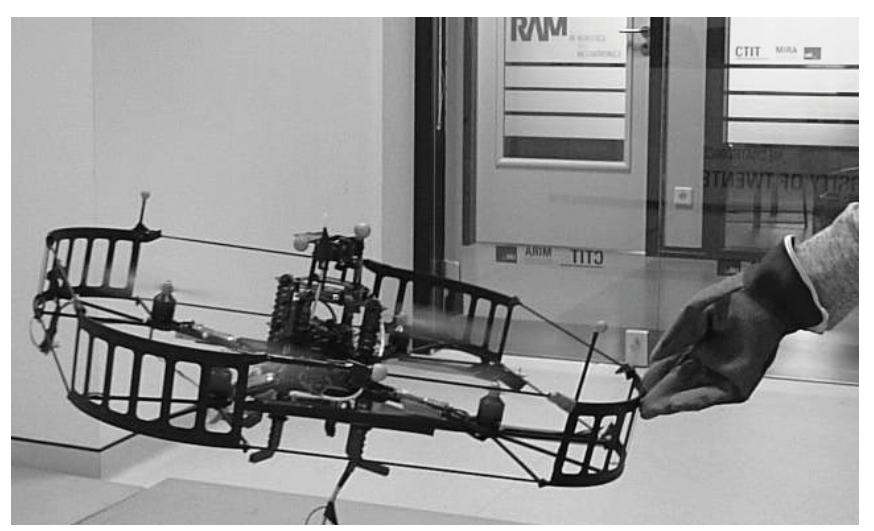

Figure 4.16: Snapshot of the experiment that involves free-flight with intermittent interaction force, applied on the quadrotor by a human.

\subsubsection{Results}

The practical applicability and effectiveness of the proposed control architecture is demonstrated in both autonomous and teleoperated free-flights and interactions. Some of the representative results are presented here.

\subsubsection{Autonomous}

For autonomous free-flight and interaction tests, the slave controller presented in Section 4.3 have been implemented on the Asctec Pelican quadrotor. The flight trajectory, impedance and desired interaction forces (when contact occurs) have been prescribed a priori.

Variable impedance in free-flight + Intermittent interaction In this experiment, a periodic trajectory in the $x y$ plane is tracked, while maintaining a constant altitude of $z=0.85 \mathrm{~m}$. In the course of this experiment, an intermittent external force is applied on the quadrotor by the human, as shown in Fig. 4.16. The intermittent forces are applied during the intervals $0-7 \mathrm{~s}, 77-79 \mathrm{~s}, 135-137 \mathrm{~s}$, and $172-174 \mathrm{~s}$. These intervals are indicated by shaded regions in the plots given in Fig. 5.11. In the course of this experiment, the stiffness of the impedance controller is changed from 50 to $100 \mathrm{~N} / \mathrm{m}$ at $t=86 \mathrm{~s}$. To illustrate the different capabilities of the proposed controller separately, the outer-loop force controller has not been activated during this experiment.

Figs. $4.17 \mathrm{a}$ and $4.17 \mathrm{~b}$ show that, in the absence of external force, the impedance controller reasonably tracks the desired lateral and longitudinal positions of the quadrotor. As theoretically expected, for the same motion profile, the tracking performance is improved when the stiffness of the impedance controller is increased, see Fig. 4.17c. When the external force is applied, only the translational dynamics along the direction of force application is affected. The affected translational dynamics behaves similar to a second order damped system. 


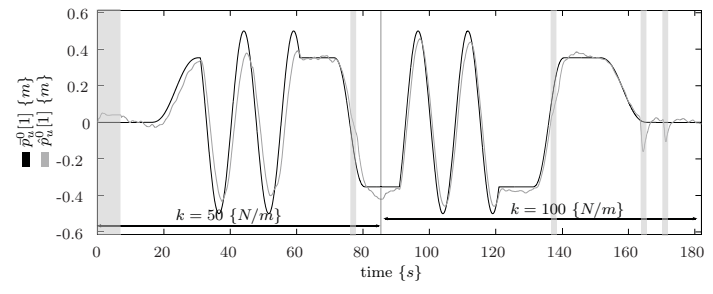

(a) Desired and actual lateral positions of the aerial robot.

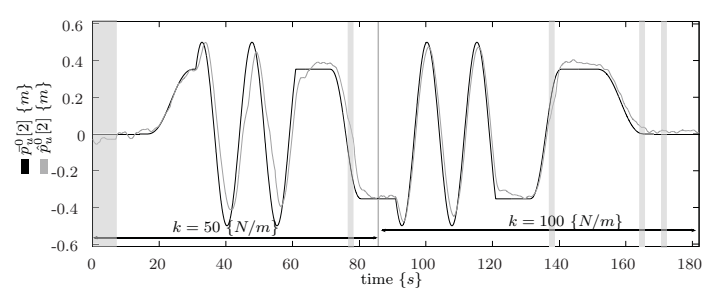

(b) Desired and actual longitudinal positions of the aerial robot.

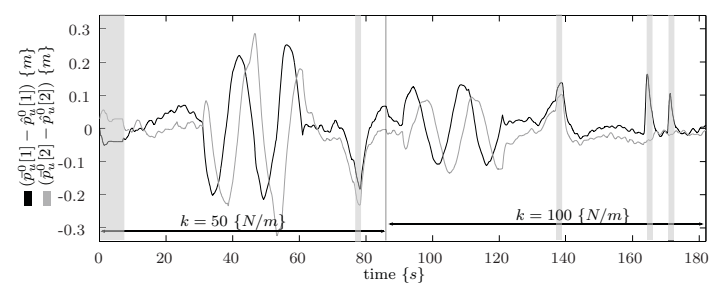

(c) Tracking errors along the lateral and longitudnal directions.

Figure 4.17: Experimental results from a task involving a periodic trajectory tracking and intermittent interaction. The time intervals during which, the quadrotor is perturbed by the external force applied by the user, are indicated by shaded regions.

Sliding on a surface This experiment involves both free-flight and interaction tasks. It shows the capability of sliding while applying a desired interaction force. The aerial robot is required to slide along the $y$ - axis, while maintaining a constant altitude of $z=0.75 \mathrm{~m}$, and applying a $1 \mathrm{~N}$ force along the $x$ - axis, when contact is detected. Unlike the assumptions in Section 4.2.2, interaction with the environment occurs at a surface, and the environment is highly nonlinear and has uneven surface. Similar to the previous experiment, the stiffness of the impedance controller is changed from 50 to $100 \mathrm{~N} / \mathrm{m}$ at $t=86 \mathrm{~s}$.

Fig. 4.18a shows the lateral position tracking performance. Once contact occurs around $t=40 \mathrm{~s}$, the position tracking performance in the direction of contact becomes poor. After the estimated external force exceeds the threshold, beyond which contact is detected, the force controller modifies the virtual position of the impedance controller, $\tilde{p}_{u}^{0}$, by superposing $\Delta p_{u}^{0}$ on the original command $\bar{p}_{u}^{0}$. The sliding motion is shown in Fig. 4.18b. As expected, the tracking performance improves as the stiffness of the impedance control increases. However, the tracking performance is lower than that obtained in the previous experiment. This is due to the unevenness of the sliding surface, which sometimes results in sudden jumps (for example, see Fig. $4.18 \mathrm{~b}$ around $t=67 \mathrm{~s}$ ). Frictional forces acting at the contact surface also affect the tracking performance. Fig. $4.18 \mathrm{c}$ shows the capability of the controller to maintain a desired interaction force while sliding on the surface. 


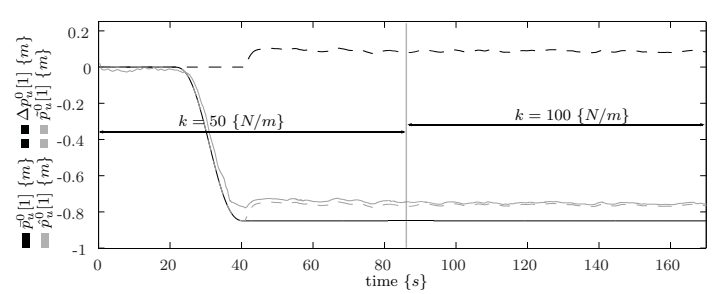

(a) Desired, modified, virtual, and actual lateral positions of the aerial robot.

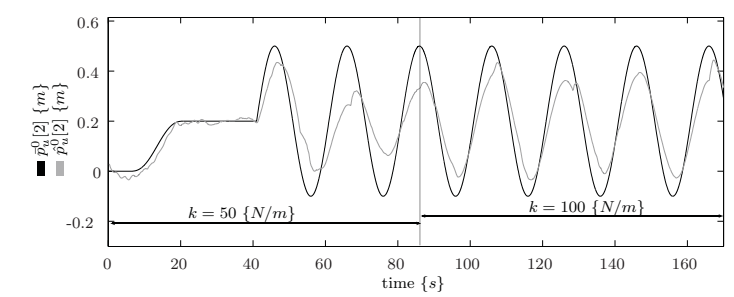

(b) Desired and actual longitudinal positions of the aerial robot.

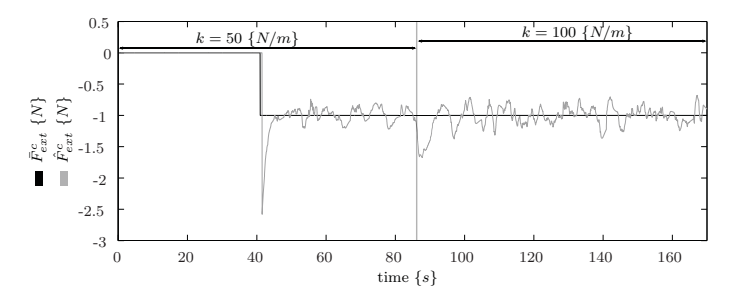

(c) Desired and actual interaction forces of the aerial robot.

Figure 4.18: Experimental results from a task involving sliding on a surface, while maintaining a constant interaction force.

Time-varying interaction force tracking In this experiment, time-varying interaction force tracking capability of the proposed controller is shown. A periodic interaction force trajectory is tracked, while almost hovering, as shown in Fig. 4.19. In the course of this experiment, the stiffness of the impedance controller is changed from 50 to $90 \mathrm{~N} / \mathrm{m}$ at $t=31 \mathrm{~s}$.

Fig. 4.20 shows the performance of the variable impedance-based interaction force regulator. As detailed out in Section 4.3, once contact is detected, the force controller modifies the virtual position of the impedance controller according to the desired time-varying interaction force. Due to the underactuation of the aerial robot, time-varying interaction forces are tracked by changing the tilting angle of the robot. The result shows a satisfactory interaction force tracking performance of the controlled aerial robot in the presence of time-varying impedance, when interacting with a non-ideal environment.

\subsubsection{Teleoperation}

The proposed control architecture has been also tested in a semi-experiment, in which the human and all hardware components, except the aerial robot and the environment, have been included. The environment and the identified dynamics of the aerial robot have been simulated in real-time Linux machine. A mapping scale of $\lambda=8$ between the master and the slave positions is used in all the teleoperation semi-experiments. 


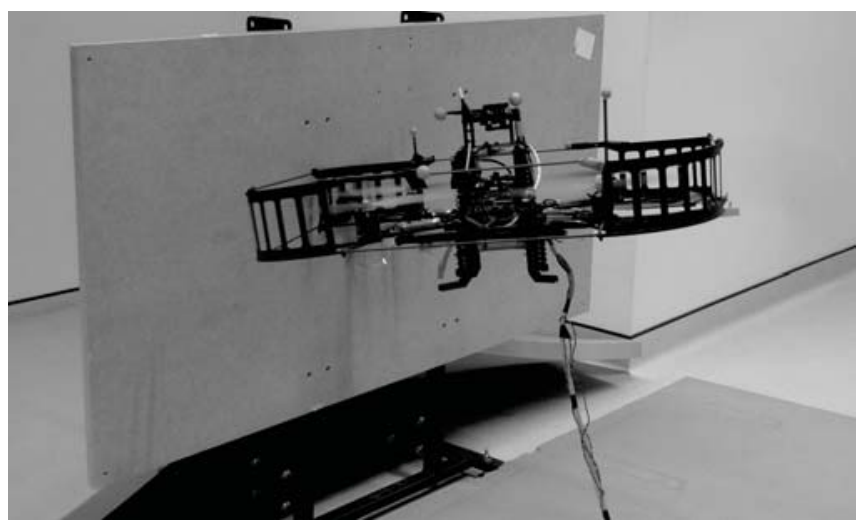

Figure 4.19: Snapshot from time-varying interaction force regulation experiment.

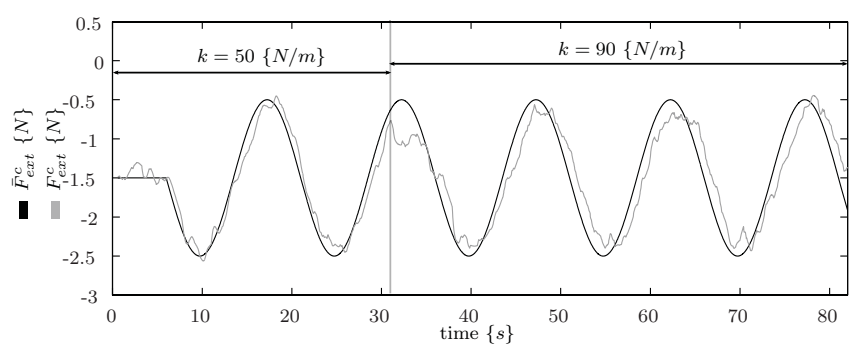

Figure 4.20: Desired and actual time-varying interaction force trajectories.

Free-flight Figs. 4.21a and 4.21b show the position trajectories and the stiffness of the slave commanded by the operator. Similar to the simulation result in Section 4.6.0.1, the tracking error during free flight is lower for higher impedance values of the slave controller and low frequency reference trajectories, see Figs. 4.21a-4.21c. Figs. 4.21b and 4.21d show the force feedback and the PWM for the linear resonant actuator vibration motor, which is responsible for the tactile feedback.

Interaction This experiment involves regulation of interaction force commanded by the operator while the aerial robot is interacting with a vertical wall. Fig. 4.22a shows the operator's force and estimate of the interaction force. As it can be seen from this figure, the time-varying operator's force, which is estimated based on the sliding-mode observer, is reasonably tracked by the force controller. Fig. 4.22b shows the time evolution of the stiffness of the impedance controller of the slave aerial robot during this experiment.

\subsection{Conclusion}

This Chapter considered the application of aerial robots for telerobotic tasks that require variable impedance and regulation of time-varying interaction force. A 


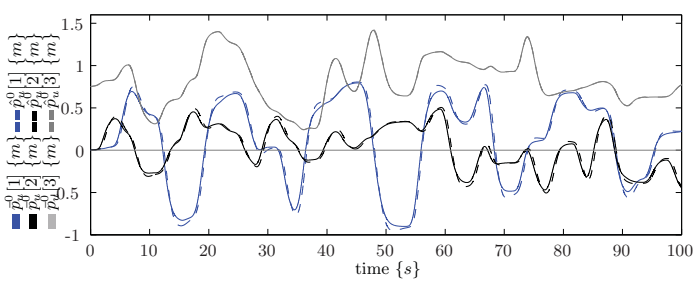

(a) Slave and scaled master positions.

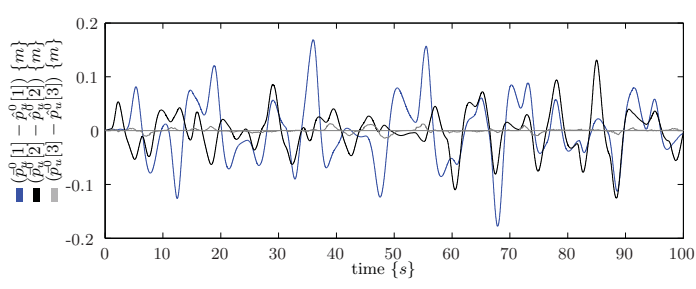

(c) Tracking error.

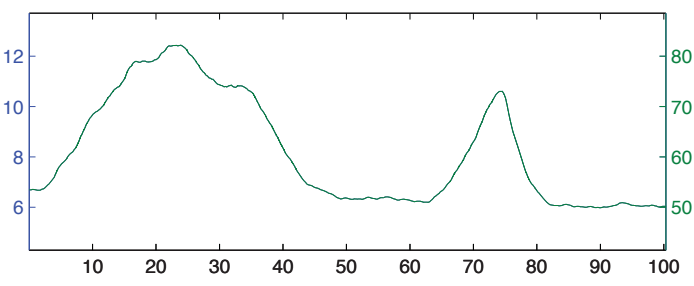

(b) The stiffness command and the PWM of the vibrotactile feedback, labeled on the left and right vertical axes, respectively.

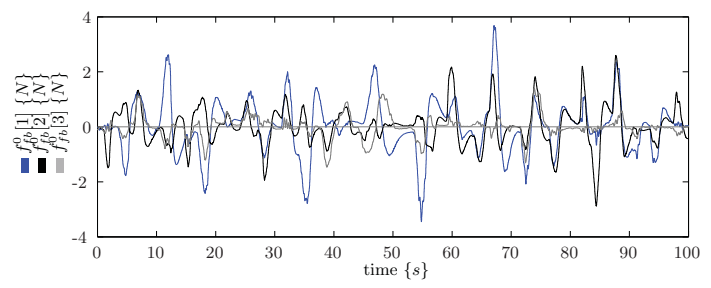

(d) Force Feedback.

Figure 4.21: Semi-Experimental results from teleoperation of the variable impedance controlled aerial robot in free-flight.

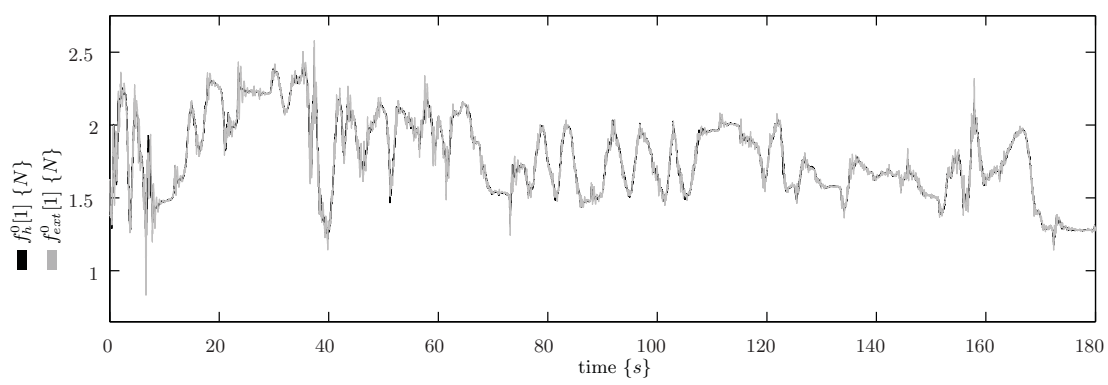

(a) The commanded operator's force and the estimated interaction force.

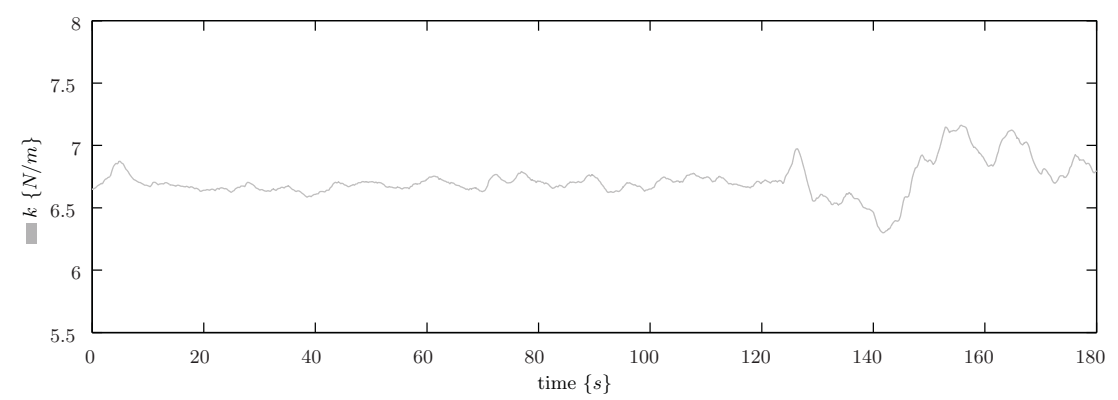

(b) The stiffness of the impedance controller.

Figure 4.22: Semi-Experimental results from teleoperation of the variable impedance controlled aerial robot in interactive task. 
versatile control architecture that consists of a variable impedance master and slave controllers, and a multimodal feedback has been proposed. Moreover, a rigorous mathematical treatment of the proposed architecture along with control tuning methods and practical considerations has been presented.

The proposed slave controller enables the deployment of aerial robots for wide range of tasks, which include docking on movable pads, regulation of forces in cooperative aerial manipulation. The proposed state observation and interaction force estimation algorithms also relieve the need for force sensors, which are, among others, costly and noise sensitive. Moreover, the multimodal feedbacks displayed to the operator have increased the situational awareness about the remotely manipulated aerial robot/environment. Extensive simulation and experimental results on different aerial platforms, in both autonomous and teleoperated tasks, corroborate all the claims made in this Chapter. 


\section{CHAPTER 5}

\section{A Control Architecture for Aerial MANipUlators}

This chapter slightly deviates from the previous chapters and presents autonomous free-flight control architecture for aerial manipulators. Recent researches in the field of aerial robotics have started developing dexterous manipulation systems, to be mounted on aerial vehicles, in order to perform task specific aerial interactions. These manipulators are often designed to be as light-weighted as possible, so that their dynamic disturbance to the flying platform is not significant. As such, classical UAV controllers can still be used without significant modification. However, such an approach, though modular, essentially limits the application only to manipulators with certain dynamics, diminishes the achievable tracking performance of the UAV, and reduces the task spectrum they are deployed in. This chapter presents a method for exploiting the dynamic influence of a manipulator as an additional actuation to control the often underactauted flying platform in free-flight. A nonlinear control law, based on the unified dynamics of the complete system, is designed. The main objective of controller is improving the tracking performance and the maneuverability of the flying platform by exploiting the dynamic influence of the manipulation system. Moreover, certain guidelines on how to efficiently exploit the dynamics of the manipulator are also provided. The achieved improved trajectory tracking performance of the proposed controller over a classical controller is illustrated both in simulation and experiments.

This chapter is an extended version of (Mersha et al. 2014b); A.Y. Mersha, S. Stramigioli, and R. Carloni, "Exploiting the dynamics of a manipulator for control of an aerial robot", In Proceedings of the IEEE International Conference on Robotics and Automation, 2014. 


\subsection{Introduction}

\subsubsection{Background}

Since the invention of UAVs, their applications have been mainly limited to tasks where no active interaction with the environment is involved. Typical applications include vision-based surveillance, forest fire detection, crop dusting, search and rescue [107]. However, recent technological advancements in the field of aerial robotics have expanded their application spectrum in the civil sector, including tasks that require active aerial interaction. Aerial inspection by contact [26], aerial grasping [91], load transportation and cooperative aerial assembling [87] are some of the resounding research achievements in aerial interaction.

UAVs, endowed with robotic manipulators and hereafter referred as aerial manipulators, have been employed for most of the aforementioned applications that require physical interaction with the environment. The appended manipulators are often designed to be light-weighted [64],[43], and are commonly placed as close to the COM of the UAV as possible [90], [64]. The primary motivations for such design and placement choices are to avoid significant change in the inertia property of the UAV and/or to keep the influence of the dynamics of the manipulator and the gravitational bias torque due to the manipulator minimal. As such, existing classical UAV controllers can still be used without major modification.

Commonly, the UAV-base controllers of aerial manipulators that have been employed as yet are variants of classical PID controllers [90], [64], [62]. They attempt to reject the static/dynamic disturbances on the UAV that are also consequences of the manipulator's motion. Loosely speaking, the role of the UAV controllers during interaction tasks is to emulate a rigid-floating base that robustly rejects disturbances including those due to the dynamics of the manipulator. On the other hand, during free-flight, the movement of the manipulator is constrained (remains static) [26], [43], and the static shift of COM and change in the rotational inertia of the aerial manipulator are possibly compensated by the integral action of the UAV's PID controller. Some works even suggested to compensate for the shift in the position of the COM by placing a counterbalancing mass on the opposite side of the UAV [42], which even increases the overall mass and rotational inertia of the complete system.

These approaches certainly limit the class of deployable aerial manipulators to those with certain mechanical configuration and dynamics. Besides, during freeflight, which is the main flight regime considered in this work, the use of classical UAV controllers for aerial manipulator results in poor tracking performance and even leads to some stability issues.

\subsubsection{Contribution}

The main contribution of this work is a free-flight control architecture for aerial manipulators based on their unified dynamics. The architecture exploits the dynamic disturbance of the manipulator on the UAV to improve the trajectory tracking 
performances of the underactuated UAV-base and to allow faster maneuvers. In this context, the chapter also proposes guidelines for the efficient exploitation of the manipulator dynamics during both mechanical and controller design phases.

The advantage of the proposed controller over classical PID controllers is twofold. First, since it is based on the unified dynamics, better tracking performance and robustness can be achieved. Second, it exploits the dynamics of a manipulator to achieve a better maneuverability. This is a new and novel approach of exploiting the dynamics of a manipulator to improve the tracking performance and increase maneuverability of an aerial manipulator by expanding its flight-envelop. The claim on the achieved better maneuverability and tracking performance of the proposed controller over the classical UAV controllers is backed up by simulations and experimental results using a quadrotor UAV on which a robotic manipulator is mounted.

The remainder of the chapter is organized as follows. Section 5.2 briefly presents the kinematic and dynamics of a benchmark planar aerial manipulator. In Section 5.3 , the state of the art in free-flight control of aerial manipulators and analysis of their limitations are provided. In Sections 5.4 and 5.5, the proposed control architecture and the stability analysis of the controlled system are presented. Illustrative simulations and experimental results are given in Sections 5.6 and 5.7, respectively. Detailed discussions about the obtained results and how to efficiently exploit the proposed architecture for related applications is provided in Section 5.8. Finally, concluding remarks are drawn in Section 5.9.

\subsection{Dynamics of an Aerial Manipulator}

Inspired by the ubiquity of quadrotors as research platforms, this section provides the kinematics and dynamics of a planar quadrotor UAV on which a 1DOF manipulator with a prismatic joint is mounted. The motivation behind using the simplified planar dynamics is to provide easy and intuitive insight about its dynamics and the proposed controller. Besides, it limits the mathematical complexity while still capturing the main features of its 3D counterpart, which is used for experimental verification.

Fig. 5.1 shows the planar aerial manipulator with labeled parameters and variables that are required to describe its kinematics and dynamics. With respect to Fig. 5.1, the following are defined:

- $\psi^{0}=\left[o_{0}, \overrightarrow{\hat{x}_{0}}, \vec{y}_{0}\right]$ and $\psi^{u}=\left[o_{u}, \overrightarrow{\hat{x}_{u}}, \overrightarrow{\hat{y}_{u}}\right]$ denote the planar inertial coordinate frame and the body-fixed coordinate frame, which is attached to the COM of the UAV.

- $c \in \mathbb{R}^{+}$is a nonzero constant lateral displacement of each propeller from $\psi^{u}$.

- $h \in \mathbb{R}^{+}$is a constant vertical displacement of the COM of the manipulator from $\psi^{u}$.

- $\ell \in \mathbb{R}$ is a lateral displacement of the COM of the manipulator from $\psi^{u}$. 


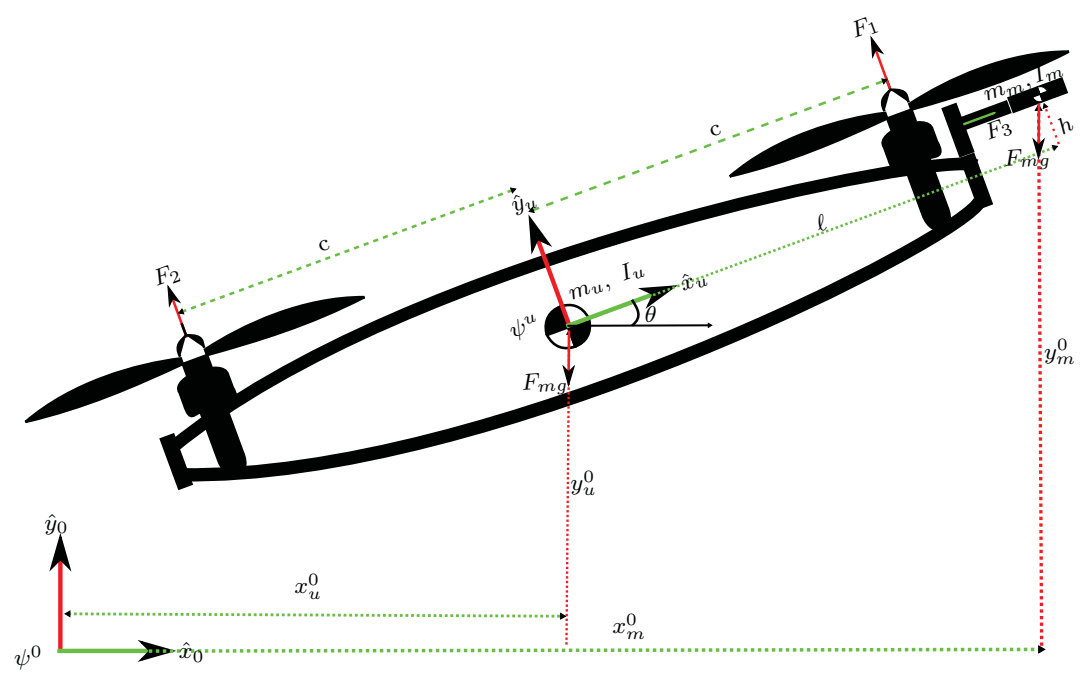

Figure 5.1: Planar aerial manipulator consisting of a planar quadrotor on which a 1D robotic manipulator with a prismatic joint is appended.

- $R_{u}^{0} \in \mathbb{R}^{2 \times 2}$ is the orthonormal rotational matrix describing the orientation of $\psi^{0}$ with respect to $\psi^{u}$, and it transforms coordinates from $\psi^{u}$ to $\psi^{0}$.

- $m$ and $I$ denote the mass and the rotational inertia of the manipulator (with subscript $m$ ) and of the planar UAV (with subscript $u$ ).

- $F_{u g}, F_{m g}$ represent the gravitational forces of the UAV and the manipulator, respectively.

- $F_{1}, F_{2}, F_{3}$ represent the actuation forces of the two propellers and of the manipulator.

Moreover, for generic vectors and matrix $x, x_{a}$ and $M$

- $x_{i}$ indicates the $i^{\text {th }}$ entry of the vector $x$.

- $x_{a_{i}}$ indicates the $i^{\text {th }}$ entry of the vector $x_{a}$.

- $m_{i j}$ indicates $i^{\text {th }}$ row and $j^{\text {th }}$ column entry of the matrix $M$.

- $M_{i j, k l}\left(M_{i j, k l}^{-1}\right)$ indicates a sub-matrix of $M\left(M^{-1}\right)$ that contains entries of $i^{\text {th }}-j^{\text {th }}$ row and $k^{\text {th }}-l^{\text {th }}$ column.

For scalars

- $\kappa_{j}^{i}$ denotes the $\kappa \in\{\theta, x, y\}$ (rotational, lateral or vertical) coordinates of the generalized configuration of $j \in\{m, u\}$ (manipulator or UAV) with respect to $i \in\{0, u\}\left(\psi^{0}\right.$ or $\left.\psi^{u}\right)$.

- $\dot{\kappa}_{j}^{k, i}$ denotes the $\kappa \in\{\theta, x, y\}$ component of the generalized velocity of $j \in\{m, u\}$ with respect to $i \in\{0, u\}$ in coordinates of $k \in\{0, u\}$. 
Let $p_{u}^{0}:=\left[\begin{array}{ll}x_{u}^{0} & y_{u}^{0}\end{array}\right]^{T}$ and $p_{m}^{u}:=\left[\begin{array}{ll}x_{m}^{u} & y_{m}^{u}\end{array}\right]^{T}=\left[\begin{array}{ll}\ell & h\end{array}\right]^{T}$, with reference to Fig. 5.1 , the following geometric relation of the generalized planar configuration of the aerial manipulator can be derived, i.e.,

$$
\left\{\begin{array}{l}
\theta_{m}=\theta_{u}=\theta \\
p_{m}^{0}=p_{u}^{0}+R_{u}^{0} p_{m}^{u}
\end{array}\right.
$$

Based on (5.1), the velocity relation is given by

$$
\left\{\begin{aligned}
\omega_{m}^{m, 0} & =\omega_{u}^{u, 0}=\dot{\theta} \\
\dot{p}_{m}^{0,0} & =\dot{p}_{u}^{0,0}+R_{u}^{0} \dot{p}_{m}^{u, u}+\dot{R}_{u}^{0} p_{m}^{u} \\
& =\dot{p}_{u}^{0,0}+\dot{\ell} R_{u}^{0}\left[\begin{array}{l}
1 \\
0
\end{array}\right]+\omega_{u}^{u, 0} R_{u}^{0} S(1) p_{m}^{u}
\end{aligned}\right.
$$

where $S(1)=\left[\begin{array}{cc}0 & -1 \\ 1 & 0\end{array}\right]$ is a skew symmetric matrix; $\dot{p}_{m}^{u, u}=\dot{\ell}[1,0]^{T}$ due to the $1 \mathrm{D}$ prismatic joint.

A unified dynamics of the complete system is derived by using the Newton-Euler approach. The considered benchmark (planar aerial manipulator) is composed of two planar rigid bodies (the manipulator and the UAV-base, each with 3DOFs) that are connected by a 1D prismatic joint. Due to this joint, the complete aerial manipulator has only 4DOFs. The first three equations describing the closed form of the dynamics of the aerial manipulator are obtained by eliminating the generalized internal forces between the two subsystems, i.e.,

$$
\begin{gathered}
m_{u} \ddot{p}_{u}^{0,0}+m_{m} \ddot{p}_{m}^{0,0}=R_{u}^{0}\left[\begin{array}{c}
0 \\
F_{1}+F_{2}
\end{array}\right]+\left[\begin{array}{c}
0 \\
F_{u g}+F_{m g}
\end{array}\right] \\
\left(I_{u}+I_{m}\right) \dot{\omega}_{u}^{u, 0}+m_{m}\left(S(1) p_{m}^{u}\right)^{T} R_{0}^{u} \ddot{p}_{m}^{0,0}=c\left(F_{1}-F_{2}\right)+\left(p_{m}^{u}\right)^{T} R_{0}^{u}\left[\begin{array}{c}
0 \\
F_{m g}
\end{array}\right]
\end{gathered}
$$

The dynamics of the fourth DOF can be obtained by resolving the translational motion of the manipulator with respect to $\psi^{0}$ along its line of motion, i.e., along $\vec{x}_{u}$,

$$
m_{m}\left[\begin{array}{ll}
1 & 0
\end{array}\right] R_{0}^{u} \ddot{p}_{m}^{0,0}=F_{3}+\left[\begin{array}{ll}
1 & 0
\end{array}\right] R_{0}^{u}\left[\begin{array}{c}
0 \\
F_{m g}
\end{array}\right]
$$

For brevity, the complete dynamics given in (5.3) - (5.5), with the addition of viscous damping, is compactly represented by

$$
M\left(x_{1}\right) \dot{x}_{2}+C\left(x_{1}, x_{2}\right) x_{2}+D x_{2}+G\left(x_{1}\right)=B\left(x_{1}\right) u
$$

where $x_{1}^{T}:=\left[\begin{array}{llll}x_{u}^{0}, & y_{u}^{0}, \quad \theta, \quad \ell\end{array}\right]$ and $x_{2}:=\dot{x}_{1} ;$ the input $u^{T}:=\left[\begin{array}{lll}F_{1} & F_{2} & F_{3}\end{array}\right]$; $M\left(x_{1}\right)=M^{T}\left(x_{1}\right), C\left(x_{1}, x_{2}\right), D, B\left(x_{1}\right)$ and $G\left(x_{1}\right)$ denote the generalized mass, centrifugal and Coriolis, viscous damping, input matrices, and the gravitational 
force vector, respectively, and are given by

$$
\begin{gathered}
M\left(x_{1}\right)=\left[\begin{array}{cccc}
m_{u}+m_{m} & 0 & -m_{m}(\ell \sin (\theta)+h \cos (\theta)) & m_{m} \cos (\theta) \\
* & m_{u}+m_{m} & m_{m}(\ell \cos (\theta)-h \sin (\theta)) & m_{m} \sin (\theta) \\
* & * & I_{u}+I_{m}+m_{m}\left(\ell^{2}+h^{2}\right) & -m_{m} h \\
* & * & * & m_{m}
\end{array}\right] \\
C\left(x_{1}, x_{2}\right)=\left[\begin{array}{cccc}
0 & 0 & -m_{m}\{2 \dot{\ell} \sin (\theta)+(\ell \cos (\theta)-h \sin (\theta)) \dot{\theta}\} & 0 \\
0 & 0 & m_{m}\{2 \dot{\ell} \cos (\theta)-(\ell \sin (\theta)+h \cos (\theta)) \dot{\theta}\} & 0 \\
0 & 0 & 2 m_{m} \ell \dot{\ell} \\
0 & 0 & -m_{m} \ell \dot{\theta} & 0
\end{array}\right] \\
D=\operatorname{diag}\left(d_{x}, d_{y}, d_{\theta}, d_{\ell}\right) \\
G\left(x_{1}\right)=\left[\begin{array}{c}
\left(m_{u}+m_{m}\right) g \\
(\ell \cos (\theta)-h \sin (\theta)) m_{m} g \\
m_{m} g \sin (\theta)
\end{array}\right], \quad B\left(x_{1}\right)=\left[\begin{array}{ccc}
-\sin (\theta) & -\sin (\theta) & 0 \\
\cos (\theta) & \cos (\theta) & 0 \\
c & -c & 0 \\
0 & 0 & 1
\end{array}\right]
\end{gathered}
$$

\subsection{Problem Formulation}

This section briefly revisits the current approaches in control of aerial manipulators during free-flight, and provides analysis on their applicability and limitations.

UAVs often have a fully actuated rotational dynamics and an underactuated translational dynamics, except the privileged axis in the direction of the thrust vector [37]. They are also characterized by coupled dynamics between their translation and rotational DOFs [80], [66]. In the considered planar aerial manipulator, there exists a strong coupling between the pitch and the lateral dynamics.

The commonly used approach to control the position of the underactuated translational DOF of such underactuated UAVs is by controlling their rotational dynamics. Hence, the effect of the manipulator on the pitch dynamics is primarily analyzed. The current state of the art in free-flight control of aerial manipulator is to keep the manipulator stationary and employ the existing UAV-base controller [64], [43], [90]. In such cases, by disregarding the effect of the translational dynamics, the pitch dynamics given by (5.4) reduces to

$$
I^{\prime} \dot{\omega}_{u}^{u, 0}+d_{\theta} \omega_{u}^{u, 0}-\left(p_{m}^{u}\right)^{T} R_{0}^{u}\left[\begin{array}{c}
0 \\
F_{m g}
\end{array}\right]=c\left(F_{1}-F_{2}\right)
$$

where $I^{\prime}:=I_{u}+I_{m}+m_{m}\left(\ell^{2}+h^{2}\right)$.

The two main approaches proposed in the literature to compensate for the static bias torque due to the action of gravity on the manipulator's mass are:

- Mechanical: mounting compensating mass on the opposite side of the UAV, [42].

- Control: leaving it for the integral controller action of the UAV's controller, [64], [90]. 


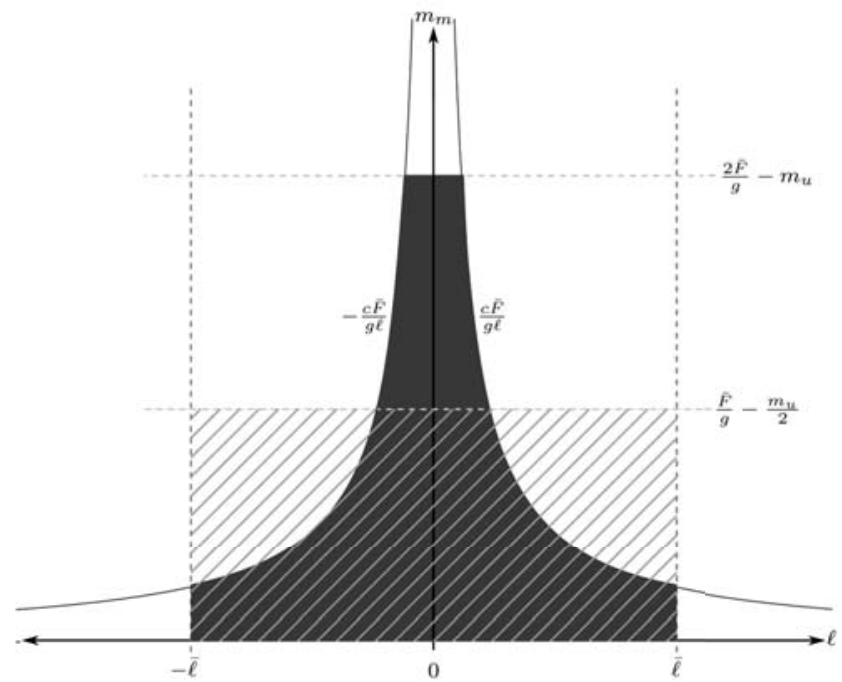

Figure 5.2: The possible combination of $m_{m}$ and $\ell$ that keeps the system statically stable. The region for the system with compensating mass is indicated by diagonal strips, while without the added mass is indicated by shade. The limits on $\ell$ are imposed by the design choice, whereas the upper bound on $m_{m}$ is as given in Table 5.1 .

These approaches are revisited here with respect to both static and dynamic stability. For the aerial manipulator to remain statically stable, the total thrust generated by the propellers should balance the action of gravity on the system. Moreover, the bias torque, due to the force of gravity on the mass of the manipulator with respect to the COM of the UAV, should be balanced by the torque generated by the total thrust. Mathematically,

Force constraint

$$
\left\{\begin{array}{cc}
\Sigma F=0=F_{1}+F_{2}-\left(m_{u}+m_{m}\right) g & \text { PID } \\
\Sigma F=0=F_{1}+F_{2}-\left(m_{u}+2 m_{m}\right) g & \text { Compensating mass + PID }
\end{array}\right.
$$

Torque constraint

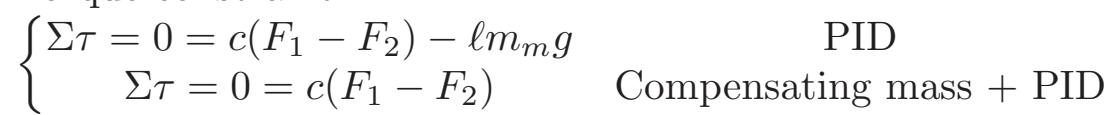

If $F_{1}, F_{2} \in[\underline{F}, \bar{F}]$ denote the thrust generated by each propeller, where $\underline{F}>0$, then, following (5.8), $m_{m}$ and $\ell$ should satisfy the constraints in Table 5.1 to remain statically stable.

Table 5.1: Constraints for Static Stability

\begin{tabular}{|c|c|c|}
\hline & Compensating mass + PID & PID \\
\hline Torque Constraint & - & $m_{m}<\frac{c \bar{F}}{g \ell}$ \\
\hline Force Constraint & $m_{m} \leq \frac{\bar{F}}{g}-\frac{m_{u}}{2}$ & $m_{m} \leq \frac{2 \bar{F}}{g}-m_{u}$ \\
\hline
\end{tabular}

Fig. 5.2 shows the possible combinations of $m_{m}$ and $\ell$ in both cases. The use of compensating mass essentially eliminates the constraint on $\ell$. However, it halves 


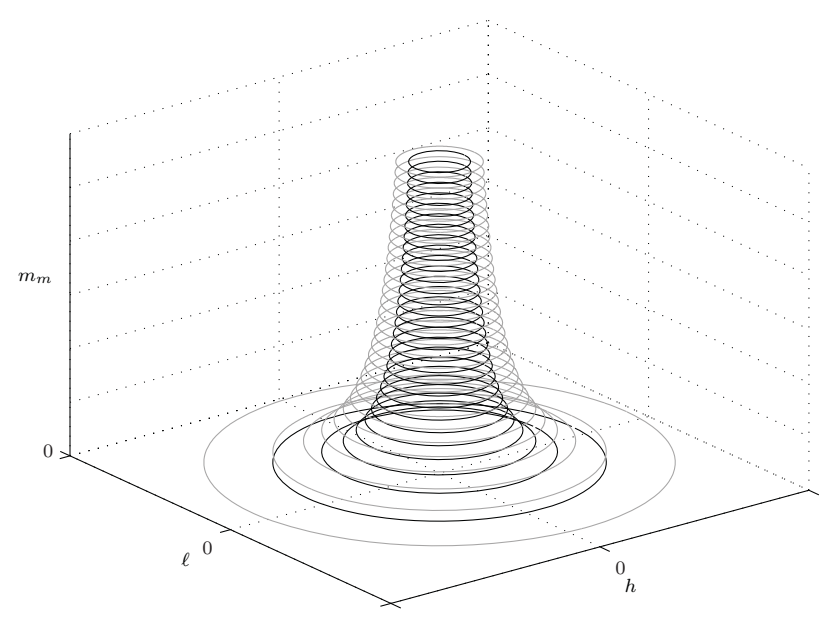

Figure 5.3: The possible combination of $m_{m}, \ell$ and $h$ that keeps the system dynamically stable. The region for the system with compensating mass is indicated by dark lines, while without the added mass is indicated by gray lines.

the maximum mass of the manipulator that can be statically supported by the thrust of the propellers compared to the case without it.

The dynamic stability of the system highly depends on the type of controller and its parameters. Assuming the input in (5.7) is within the propellers' capability, the stability boundary of the ubiquitous PID controller is explored. Consider a PID controller with structure $\left\{k_{P}+\frac{1}{s} k_{I}+s k_{D}\right\}$ and positive constants; then $m_{m}$ and $\ell$ should satisfy the constraint given in Table 5.2 to remain dynamically stable. ${ }^{1}$

Table 5.2: Constraints for Dynamic Stability

\begin{tabular}{|c|c|}
\hline Compensating mass + PID & PID \\
\hline$\ell^{2}+h^{2} \leq \frac{1}{2 m_{m}} \frac{k_{P}\left(d_{\theta}+k_{D}\right)}{k_{I}}$ & $\ell^{2}+h^{2} \leq \frac{1}{m_{m}} \frac{k_{P}\left(d_{\theta}+k_{D}\right)}{k_{I}}$ \\
\hline
\end{tabular}

Fig. 5.3 shows the region of possible combinations of $m_{m}, \ell$ and $h$, for which the system with and without compensating mass will remain stable under the same PID controller designed for the UAV-base alone. This result is similar to [90] for added load on a miniaturized helicopter. It is clear from the figure that the added compensating mass further limits the range of allowable parameters of the manipulator compared to its counterpart without it.

For a given UAV-base, the region in Fig 5.3 can be widened by tuning the PID controllers in such a way that the controlled system has large stability margins. However, pushing the limit of the PID controller for a wider range of parametric disturbance or bias may result in unacceptable deterioration in performance.

The tracking performance is dependent on the bandwidth, which can be computed from the closed loop system obtained by the PID controller and (5.7). Fig. 5.4 shows the effect of the inertia of the manipulator and the compensating mass

\footnotetext{
${ }^{1}$ The expression given in Table 5.2 is obtained by disregarding $I_{u}$ (often it is relatively insignificant) and applying the Routh-Hurwitz stability criterion on the closed loop dynamics of the system.
} 


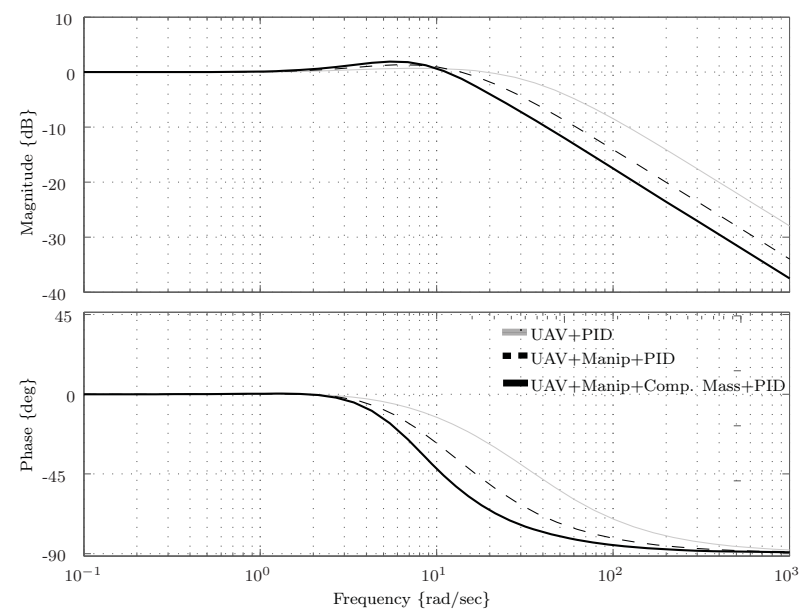

Figure 5.4: Effect of the manipulator and the compensating mass inertia on the bandwidth of the controlled aerial manipulator.

on the bandwidths of the closed loop system. The higher the added inertia, the lower the bandwidth of the controlled aerial manipulator becomes. It is worth noting that lower bandwidth on the pitch dynamics may have consequences on the stability of the complete system. Since a cascaded control approach is usually adopted to address the underactuation of UAVs, poor tracking performance in the pitch dynamics may even result in loss of stability in the lateral dynamics.

The proposed controller, whose design is presented in detail in the sequel, addresses the above limitations. In particular, it improves the stability robustness without compromising the performance of the complete system.

\subsection{Controller Design}

In this section, the proposed nonlinear controller design is presented. The controller relies on an input-output linearization and stable zero dynamics. To facilitate the control design, the standard state space representation of the dynamics of the planar aerial manipulator is used.

Let the states of the system be $x^{T}:=\left[\begin{array}{ll}x_{1}^{T} & x_{2}^{T}\end{array}\right] \in \mathbb{R}^{8}$. The state space representation of the dynamics given by (5.6) can be written as

$$
\left\{\begin{array}{c}
\overbrace{\left[\begin{array}{c}
\dot{x}_{1} \\
\dot{x}_{2}
\end{array}\right]}^{\dot{x}}=\overbrace{\left[\begin{array}{l}
x_{2} \\
-M^{-1}\left(x_{1}\right)\left\{C\left(x_{1}, x_{2}\right) x_{2}+D x_{2}+G\left(x_{1}\right)\right\}
\end{array}\right]}^{f(x)}+\overbrace{\left[\begin{array}{c}
0 \\
M^{-1}\left(x_{1}\right) B\left(x_{1}\right)
\end{array}\right]}^{g(x)} u \\
y=\left[\begin{array}{lll}
x_{u}^{0} & y_{u}^{0} & \ell
\end{array}\right]^{T}
\end{array}\right.
$$

where $f(x)$ and $g(x)$ are the drift and input vector fields, respectively; $h(x)$ is a vector of smooth functions; $u, y \in \mathbb{R}^{3}$ are the input and output vectors of the system. 


\subsubsection{Feedback Linearization}

The feedback linearization procedure transforms the nonlinear dynamic behavior of the system to a linear one by means of nonlinear feedback. As such, any preferred linear controller is designed for the feedback linearized system [83], [40].

It is easily verifiable that the dynamics of the system described by (5.9) is flat with respect to the flat output vector $y$ [56], [63], implying that the system is dynamic feedback linearizable. However, it is not static feedback linearizable with respect to $y$, [83]. Nonetheless, static feedback linearization approach and cascaded control strategy, [80], [66], is chosen over dynamic feedback linearization to reduce the complexity of the controller. With the aim of ultimately controlling $y$, an intermediate output vector $z^{T}=\left[\begin{array}{lll}y_{u}^{0} & \theta & \ell\end{array}\right]$ is defined. The planar aerial manipulator has the following properties with respect to $z$ :

i) It has a relative degree vector $r=\left[\begin{array}{lll}r_{1} & r_{2} & r_{3}\end{array}\right]$, where

$$
L_{g_{j}} L_{f}^{k} h_{i}(x)=0
$$

$L$ is the Lie derivative, $\forall j|1 \leq j \leq 3, \forall k| k<r_{i}-1, \forall i \mid 1 \leq i \leq 3$. For the above system, $r_{i}=2$.

ii) The $3 \times 3$ matrix given by

$$
\alpha(x)=\left(\begin{array}{lll}
L_{g_{1}} L_{f} h_{1}(x) & L_{g_{2}} L_{f} h_{1}(x) & L_{g_{3}} L_{f} h_{1}(x) \\
L_{g_{1}} L_{f} h_{2}(x) & L_{g_{2}} L_{f} h_{2}(x) & L_{g_{3}} L_{f} h_{2}(x) \\
L_{g_{1}} L_{f} h_{3}(x) & L_{g_{2}} L_{f} h_{3}(x) & L_{g_{3}} L_{f} h_{3}(x)
\end{array}\right)=M_{24,14}^{-1}\left(x_{1}\right) B\left(x_{1}\right)
$$

has a rank $(\alpha(x))=3, \forall x \in \mathbb{R}^{8} \mid\{c=0\}$, where $c$ is the displacement from the geometric center of the quadrotor to the propellers. Since $c \neq 0, \alpha(x)$ is always invertible.

Based on properties i) \& ii), it can be deduced that the system dynamics is input-output ( $z$ as output) linearizable with

$$
u=\alpha^{-1}\left(x_{1}\right)\left(-\beta\left(x_{1}, x_{2}\right)+v\right)
$$

where,

$$
\beta\left(x_{1}, x_{2}\right)=-M_{24,14}^{-1}\left(x_{1}\right)\left(C\left(x_{1}, x_{2}\right) x_{2}+D x_{2}+G(x)\right)
$$

$v \in \mathbb{R}^{3}$ is a new input vector to the feedback linearized system. After the linearization, (5.9) reduces to

$$
\left[\begin{array}{c}
\dot{x}_{1} \\
\dot{x}_{2_{1}} \\
\dot{x}_{2_{2,4}}
\end{array}\right]=\left[\begin{array}{c}
x_{2} \\
\varrho\left(x_{1}, x_{2}, v\right) \\
v
\end{array}\right]
$$

where,

$$
\begin{aligned}
\varrho\left(x_{1}, x_{2}, v\right)= & -\left\{v_{1}+g+\frac{d_{y}}{m_{u}+m_{m}} \dot{x}_{1_{2}}\right\} \tan \left(x_{1_{3}}\right) \\
& -\frac{1}{m_{u}+m_{m}}\left\{d_{x} \dot{x}_{1_{1}}+\frac{m_{m}}{\cos \left(x_{1_{3}}\right)}\left(v_{3}-h v_{2}-\ell \dot{x}_{1_{3}}^{2}\right)\right\}
\end{aligned}
$$


Since the dimension of the state is 8 and its total relative degree is 6, (5.9) is partially linearizable. This is also evident in (5.10). The remaining two states, $x_{1_{1}}$ and $x_{2_{1}}$, belong to the internal dynamics. Now, the zero dynamics of the system can be obtained by computing the control input $v$ that results in $z=0$, i.e., $z=0 \Rightarrow v=0$. Hence, the system's zero dynamics is described by

$$
\left\{\begin{array}{l}
\dot{x}_{1_{1}}=x_{2_{1}} \\
\dot{x}_{2_{1}}=-\frac{d_{x}}{m_{u}+m_{m}} x_{2_{1}}
\end{array}\right.
$$

Clearly, (5.11) is a linear system, whose eigen values are $\lambda_{1}=-\frac{d_{x}}{m_{u}+m_{m}}$ and $\lambda_{2}=0$. Thus, the zero dynamics of the planar aerial manipulatoris stable.

\subsubsection{State Feedback and Feed-forward Controller}

To achieve a good tracking performance, a combination of state feedback and feedforward controller can be designed for the decoupled linear dynamics of the last three DOFs of the feedback linearized system, i.e., $\left[\begin{array}{lll}y_{u}^{0} & \theta & \ell\end{array}\right]$. It follows that

$$
v=\dot{\bar{x}}_{2_{2,4}}+K_{d}\left(\bar{x}_{2_{2,4}}-x_{2_{2,4}}\right)+K_{p}\left(\bar{x}_{1_{2,4}}-x_{1_{2,4}}\right)
$$

where $K_{p}=\operatorname{diag}\left(k_{p y}, k_{p \theta}, k_{p \ell}\right), K_{d}=\operatorname{diag}\left(k_{d y}, k_{d \theta}, k_{d \ell}\right) \in \mathbb{R}^{3 \times 3}$ are positive definite diagonal matrices representing the proportional and derivative gains of the feedback controller; the bar indicates the desired reference of the respective states.

Because of the underactuation of the system, the lateral dynamics in the present form cannot be controlled directly. However, control of the lateral position is of primary importance in most free-flights. To address this problem, $\bar{x}_{1_{3}}$ is generated in such a way that the desired lateral trajectory is tracked. Under a reasonable assumption that the manipulator and pitch dynamics are time scale separated from the lateral dynamics, it follows that

$$
\dot{x}_{2_{1}} \approx \varrho\left(x_{1}, x_{2}, v_{1}\right)
$$

where

$$
\varrho\left(x_{1}, x_{2}, v_{1}\right)=-\left(v_{1}+g+\frac{d_{y}}{m_{u}+m_{m}} \dot{x}_{1_{2}}\right) \tan \left(x_{1_{3}}\right)-\frac{d_{x}}{m_{u}+m_{m}} \dot{x}_{1_{1}}
$$

Now, $\varrho\left(x_{1}, x_{2}, v_{1}\right)$ can be considered as a virtual input to the lateral dynamics. To track a lateral reference trajectory, the desired virtual input $\bar{\varrho}\left(x_{1}, x_{2}, v_{1}\right)$ can be designed as

$$
\bar{\varrho}\left(x_{1}, x_{2}, v_{1}\right)=\dot{\bar{x}}_{2_{1}}+k_{d}\left(\bar{x}_{2_{1}}-x_{2_{1}}\right)+k_{p}\left(\bar{x}_{1_{1}}-x_{1_{1}}\right)
$$

For the pitch dynamics, the reference trajectory $\bar{x}_{1_{3}}$ can be uniquely extracted from (5.10) and (5.14) with additional constraints of $\left\|\bar{x}_{1_{3}}\right\|_{\infty}<\frac{\pi}{2}$ (to avoid overturning) and $\underline{v}_{1}>-g$ (since the aerial robot considered has only unidirectional thrust).

To increase the robustness of the designed controller against measurement error, parametric uncertainties and static disturbances, saturated integral control action can be introduced to the control laws given in (5.12) and (5.14). 


\subsubsection{Manipulator Trajectory Planning}

It has been shown in the previous subsections that the manipulator's position $\left(x_{1_{4}}=\ell\right)$ can be independently controlled, see (5.10) and (5.12). But from a task viewpoint, free-flight in this case, the main DOFs are the vertical and lateral dynamics. Thus, the manipulator's DOF, which is usually constrained during freeflight, can be exploited for secondary tasks.

While this extra DOF can be exploited for a wide range of secondary tasks, here the dynamics of this DOF is exploited to achieve a better maneuverability by expanding the flight envelope of the aerial manipulator with respect to its constrained counterpart. Since the pitch dynamics is very crucial for control of the overall system, due to the underactuation, it is proposed to generate the manipulator's path based on the desired pitch acceleration $\left(\dot{\bar{x}}_{2_{3}}=\ddot{\theta}_{d}\right)$.

With an eye on Fig. 5.1, the path is generated to move the COM of the robotic manipulator in order to influence the pitch dynamics by

- changing the rotational inertia of the complete system

- modifying the influence of gravitational torque

- exploiting the toque due to the actuator force on the prismatic joint

A $5^{\text {th }}$ order polynomial function is used to generate a smooth trajectory up to its second derivative, fulfilling 6 constraints, $\bar{x}_{1_{4}}\left(t_{i}\right)=x_{1_{4}}\left(t_{i}\right), \bar{x}_{2_{4}}\left(t_{i}\right)=x_{2_{4}}\left(t_{i}\right)$, $\dot{\bar{x}}_{2_{4}}\left(t_{i}\right)=\dot{x}_{2_{4}}\left(t_{i}\right)$ and $\bar{x}_{1_{4}}\left(t_{f}\right)=\ell \in[\underline{\ell}, \bar{\ell}], \bar{x}_{2_{4}}\left(t_{f}\right)=0, \dot{\bar{x}}_{2_{4}}\left(t_{f}\right)=0$; where $t_{i}$ and $t_{f}$ denote the initial and final time.

$$
\bar{x}_{1_{4}}(t)=a_{5} t^{5}+a_{4} t^{4}+a_{3} t^{3}+a_{2} t^{2}+a_{1} t+a_{0}
$$

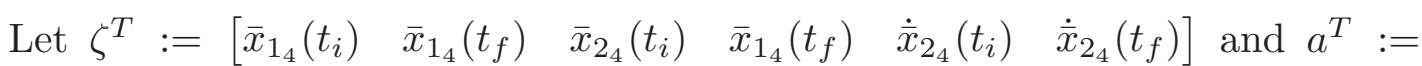
$\left[\begin{array}{llllll}a_{5} & a_{4} & a_{3} & a_{2} & a_{1} & a_{0}\end{array}\right]$, then the coefficients of the polynomial function $a_{i}$, for $i=1,2,3,4,5$, can be computed as

$$
a=\delta\left(t_{i}, t_{f}\right) \zeta
$$

where $\delta \in \mathbb{R}^{6 \times 6}$ is a mapping matrix obtained by evaluating (5.15) and its first and second derivatives at $t_{i}$ and $t_{f}$.

In the path generation, $t_{f}=t_{i}+\Delta$, where $\Delta$ is a variable through which the rate of change of the path is related to $\dot{\bar{x}}_{2_{3}}$. In this work, $\Delta$ is selected from set of possible values that are inversely proportional to $\dot{\bar{x}}_{2_{3}}$, i.e., the higher the desired pitch acceleration, the faster the desired change in the position of the COM of the manipulator becomes.

Fig. 5.5 shows the complete schematic representation of the proposed controller. The cascaded feature of the controlled system is clearly visible in the figure.

Remark 5.1 Due to actuator saturation, not all reference trajectories can be faithfully tracked with a desired rate of convergence. Therefore, infeasible references that lead to actuator saturation are modified to feasible references. 


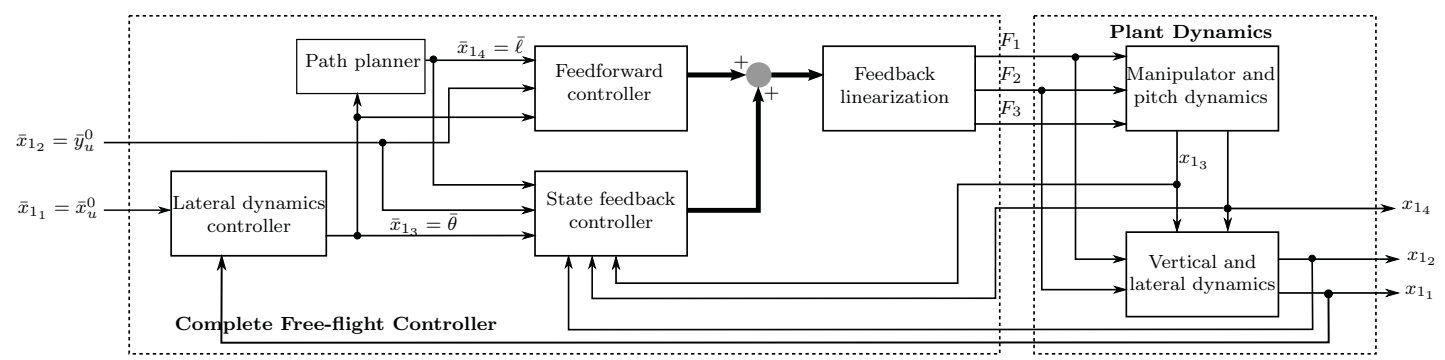

Figure 5.5: The proposed free-flight controller is based on the unified dynamics of the aerial manipulator that exploits the dynamics of the robotic manipulator mounted on it. The labeled signals also represent their first and second time derivatives. The states with and without the bar indicate the reference and actual states, respectively.

\subsection{Stability Analysis}

The stability of the aerial manipulator with the proposed controller can be shown in two steps. First, the stability of the two subsystems (the feedback linearizable and the internal dynamics) is shown independently. Later, the stability of their interconnection dynamics is presented.

\subsubsection{Stability of the Feedback Linearizable Dynamics}

As shown in Section 5.4.1, the feedback linearizable dynamics of the aerial manipulator are the $y_{u}^{0}, \theta$ and $\ell$ dynamics. Using the intermediate output $z$, define the error vector $e_{1}:=\bar{z}-z$, and its rate $e_{2}:=\dot{e}_{1}$. With reference to (5.10) and (5.12), the error dynamics of the controlled linearizable part of the system is then given by

$$
\left(\begin{array}{c}
\dot{e}_{1} \\
\dot{e}_{2}
\end{array}\right)=\left(\begin{array}{cc}
0_{3 \times 3} & I_{3 \times 3} \\
-K_{p} & -K_{d}
\end{array}\right)\left(\begin{array}{l}
e_{1} \\
e_{2}
\end{array}\right)
$$

where $I_{3 \times 3}$ and $0_{3 \times 3}$ are $3 \times 3$ identity and zero matrices.

Proposition 5.1 The control input of the system $u$ given in Sec. 5.4.1, in which the intermediate control input $v$ is defined by (5.12), exponentially stabilizes the vertical, rotational and manipulator dynamics of the aerial manipulator.

Proof: Direct substitution of $u$ and $v$ in (5.9) results in an error dynamics given in (5.17), whose system matrix is Hurwitzian as long as $K_{p}$ and $K_{d}$ are positive definite matrices. Hence, $e_{1}$ and $e_{2}$ exponentially converge to zero, ensuring the tracking of a desired trajectory.

Remark 5.2 If an integral action with integral constant $K_{i}=\operatorname{diag}\left(k_{i y}, k_{i \theta}, k_{i \ell}\right)$ is appended to (5.12), Proposition 5.1 still holds if the entries of $K_{i}$ are tuned as $k_{i j}>0$ and $k_{i j}<k_{p j} k_{d j}$, for $j \in\{y, \theta, \ell\}$. 


\subsubsection{Stability of the Lateral Dynamics}

Assume $\bar{\varrho}\left(x_{1}, x_{2}, v_{1}\right)=\varrho\left(x_{1}, x_{2}, v_{1}\right)$, substituting $\bar{\varrho}\left(x_{1}, x_{2}, v_{1}\right)$ given by $(5.14)$ in the lateral dynamics of (5.10) yields

$$
\left(\begin{array}{c}
\dot{e}_{3} \\
\dot{e}_{4}
\end{array}\right)=\overbrace{\left(\begin{array}{cc}
0 & 1 \\
-k_{p x} & -k_{d x}
\end{array}\right)}^{A}\left(\begin{array}{l}
e_{3} \\
e_{4}
\end{array}\right)+\overbrace{\left(\begin{array}{l}
0 \\
1
\end{array}\right)}^{b} \gamma
$$

where $e_{3}:=\bar{x}_{1_{1}}-x_{1_{1}}, e_{4}:=\dot{e}_{3}$ and $\gamma:=\varrho\left(x_{1}, x_{2}, v\right)-\bar{\varrho}\left(x_{1}, x_{2}, v_{1}\right)$.

Proposition 5.2 If $\gamma \longrightarrow 0$, the virtual control input $\varrho\left(x_{1}, x_{2}, v_{1}\right)$ exponentially stabilizes the lateral dynamics.

Proof: $\quad \gamma \longrightarrow 0 \Leftrightarrow \varrho\left(x_{1}, x_{2}, v\right) \approx \bar{\varrho}\left(x_{1}, x_{2}, v_{1}\right)$. Then, substituting $\bar{\varrho}\left(x_{1}, x_{2}, v_{1}\right)$ in the lateral dynamics of (5.10) results in an autonomous error dynamics with system matrix $A$ given by (5.18). $A$ is Hurwitzian provided that $k_{p x}, k_{d x}>0$. As a result, $e_{3}$ and $e_{4}$ exponentially converge to zero, ensuring the tracking of a desired lateral trajectory.

Remark 5.3 Intuitively, $\gamma \longrightarrow 0$ can be achieved by tuning the control parameters of the pitch and manipulator dynamics in a way that their dynamics is faster than the lateral dynamics.

Proposition 5.3 If $\gamma \neq 0$ and $\|\gamma\|_{\infty}<\sigma<\infty$, $\bar{\varrho}\left(x_{1}, x_{2}, v_{1}\right)$ results in bounded input bounded state (BIBS) stable system. Alternatively stated, the resulting $d y$ namics in (5.18) is Input-State stable (ISS) [1].

Proof: If $\gamma \neq 0$, substituting $\bar{\varrho}\left(x_{1}, x_{2}, v_{1}\right)$ in the lateral dynamics results in a linear error dynamics given by (5.18), with input $\gamma$. Using the well known solution of first order linear system, the requirement on the system's ISS is equivalently translated as, by abuse of notation, the error trajectories $\left(e=\left[\begin{array}{ll}e_{3} & e_{4}\end{array}\right]^{T}\right)$ fulfilling the following condition [1]

$$
|\mathbf{e}(t)| \leq\left\|e^{A t}\right\| e(0)+\left(\|b\| \int_{0}^{\infty} e^{A s} d s\right)\|\gamma\|_{\infty}
$$

It is trivial to observe that the above inequality always holds because $A$ is Hurwitzian.

Remark 5.4 Considering the fact that the system has saturated actuator inputs and $\left\|x_{1_{3}}\right\|_{\infty}<\frac{\pi}{2} \Rightarrow 0<\left|\cos \left(x_{1_{3}}\right)\right|<1$, the assumption that $\|\gamma\|_{\infty}<\sigma$ is a mild restriction on $x_{2_{3}}^{2}$ being bounded. 


\subsubsection{Stability of the Cascaded System}

To show the stability of the two connected systems, one needs to observe the interconnection dynamics. Due to mainly the assumption that $\bar{\varrho}\left(x_{1}, x_{2}, v_{1}\right) \approx$ $\varrho\left(x_{1}, x_{2}, v_{1}\right)$, the controlled lateral dynamics given by $(5.18)$ is perturbed by $\varrho=$ $\varrho\left(x_{1}, x_{2}, v_{1}\right)-\bar{\varrho}\left(x_{1}, x_{2}, v_{1}\right)$. As a result, the lateral error dynamics becomes

$$
\left(\begin{array}{c}
\dot{e}_{3} \\
\dot{e}_{4}
\end{array}\right)=\left(\begin{array}{cc}
0 & 1 \\
-k_{p x} & -k_{d x}
\end{array}\right)\left(\begin{array}{l}
e_{3} \\
e_{4}
\end{array}\right)+\left(\begin{array}{l}
0 \\
1
\end{array}\right) \gamma+\left(\begin{array}{l}
0 \\
1
\end{array}\right) \tilde{\varrho}
$$

Based on the growth rate conditions for stability of cascaded systems, provided in [88], and following similar procedures as in [89], it can be shown that $e_{3}$ and $e_{4}$ are clearly defined and there exists a finite time $t \in \mathbb{R}$ in which $\tilde{\varrho} \longrightarrow 0$.

\subsection{Simulations}

In this section, simulation results that validate the performance of the proposed controller are presented. The system model and control parameters used in the simulation are given in Table 5.3.

Table 5.3: Model and Control Parameters \{in SI units\}.

\begin{tabular}{|c|c|}
\hline \multicolumn{2}{|c|}{ Planar Aerial Manipulator parameters } \\
\hline$m_{u}=1.3, m_{m}=0.2$ & $I_{u}=0.15, I_{m}=0.005$ \\
\hline$c=0.2, h=0.05$ & $g=9.8, \ell \in[0.2$ \\
\hline \multicolumn{2}{|c|}{ Control Parameters } \\
\hline $\bar{F}_{1}=\bar{F}_{1}=12$ & $\bar{F}_{3}=5$ \\
\hline \multicolumn{2}{|c|}{ Cons } \\
\hline$k_{(p x)}=k_{(p y)}=24.5$ & $k_{(p \theta)}=400, k_{(p \ell)}=1000$ \\
\hline$k_{(d x)}=k_{(d y)}=10$ & $k_{(d \theta)}=210, k_{(d \ell)}=490$ \\
\hline$k_{(i x)}=k_{(i y)}=8$ & $k_{(i \theta)}=k_{(i \ell)}=100$ \\
\hline
\end{tabular}

\subsubsection{Tracking Performance and Robustness}

In this simulation, it is shown that a desired lateral and vertical trajectories are robustly tracked by the proposed controller in the presence of initial measurement error, random Gaussian measurement noise, and parametric uncertainties. ${ }^{2}$ Fig. $5.6 \mathrm{a}$ shows the reference and actual states of the planar aerial manipulator. It can be seen in Fig. 5.6b that the tracking error is larger at the beginning mainly due to initial measurement error and it quickly converges to nearly zero. It can be seen that the desired trajectories are robustly tracked despite the presence of parametric uncertainties and measurement errors. Fig. 5.7 shows the applied input forces during this task.

\footnotetext{
${ }^{2}$ A suitable state variable filter has been implemented to filter out significant part of the measurement noise.
} 

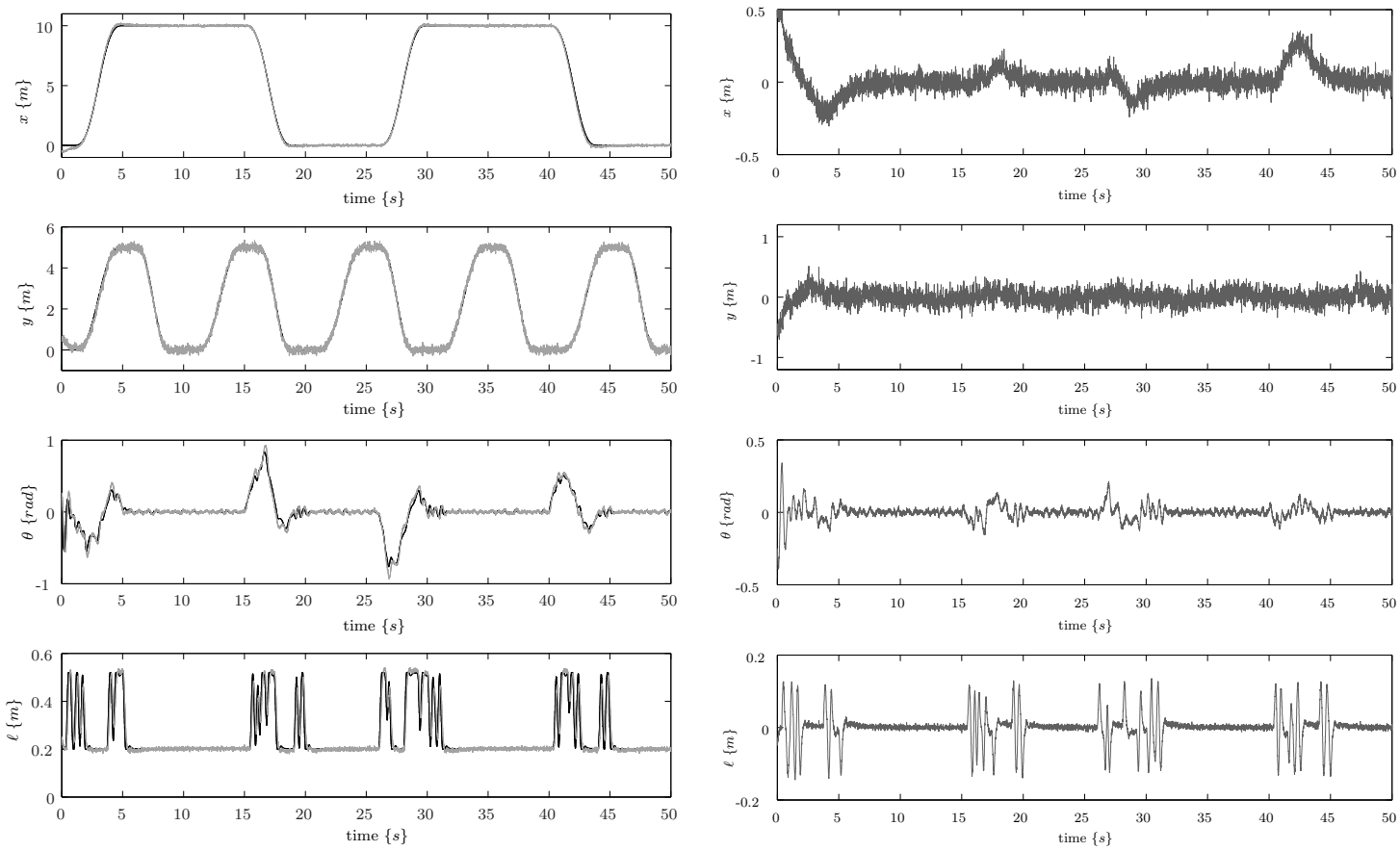

(a) States of the planar aerial manipulator. The references are indicated by dark lines, whereas

(b) Error in tracking. the actual states are indicated by gray.

Figure 5.6: Tracking performance of the proposed controller.

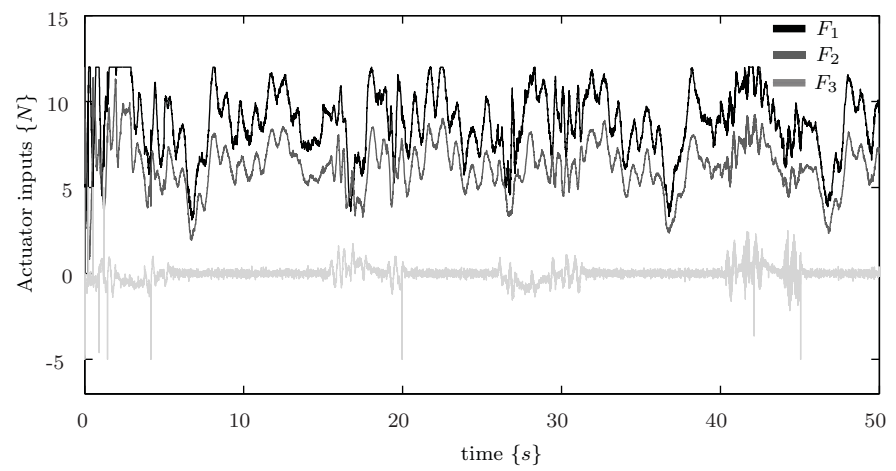

Figure 5.7: Actuator inputs.

\subsubsection{Maneuverability Comparison}

This simulation compares the achievable maneuverability of the aerial manipulator using different tracking controllers and position of the manipulator. Due to the underactuation of the lateral dynamics and its strong coupling with the pitch dynamics, aggressive correction of error in the pitch trajectory is fundamental for the control of the lateral dynamics. Thus, the comparison is made by particularly observing the pitch dynamics. 


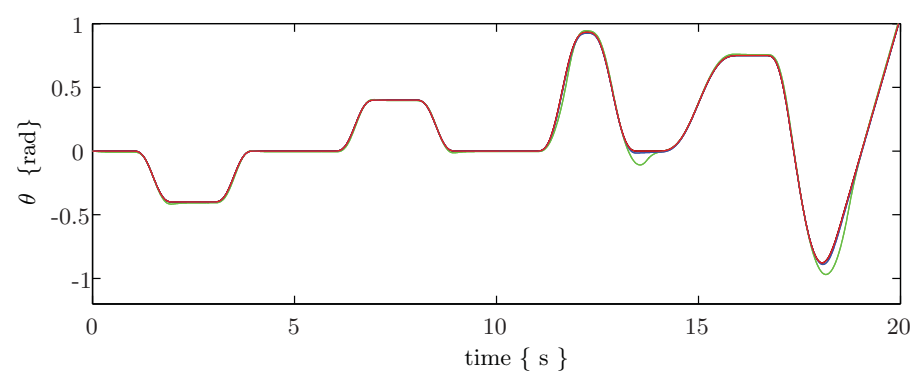

(a) Pitch trajectory

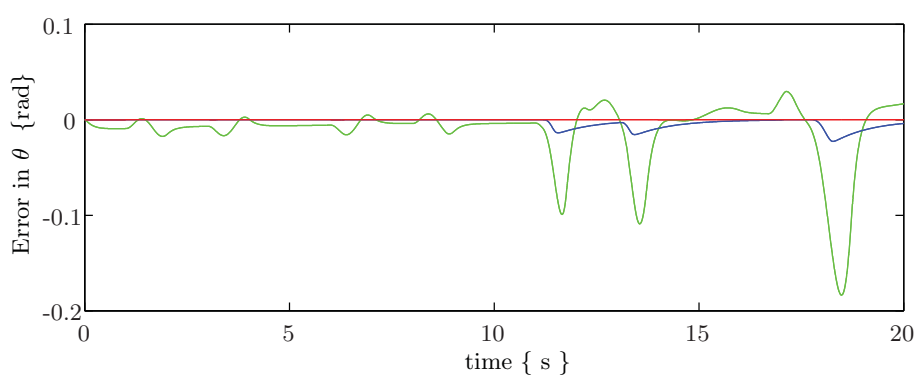

(b) Pitch tracking error

Figure 5.8: Comparison of reference pitch tracking capabilities of different controllers. PID controller (green), the proposed controller with the motion of the manipulator constrained at $\frac{\bar{\ell}+\underline{\underline{\ell}}}{2}$ (blue), and the controller that exploits the dynamics of the manipulator (red).

The comparison is made between the classical PID controller, the proposed controller with the motion of the manipulator constrained at the center, i.e., $\frac{\bar{\ell}+\underline{\ell}}{2}$, and the controller that exploits the dynamics of the manipulator. Fig. 5.8a and $5.8 \mathrm{~b}$ show the performance of the three controllers while tracking a representative desired pitch trajectory. As can be seen in the figure, the controllers designed based on the unified dynamics of the aerial manipulator outperform the PID controller in the entire trajectory. However, in the second half of the trajectory where the maneuver requires more input torque than that can be delivered by the thrust alone, the performance of the proposed controller that exploits the dynamics of the manipulator is better than the rest. It can be seen in Fig. 5.9 that by exploiting the dynamics of the robotic manipulator, the thrust required for maneuvering is less than required by the other controllers for the same trajectory.

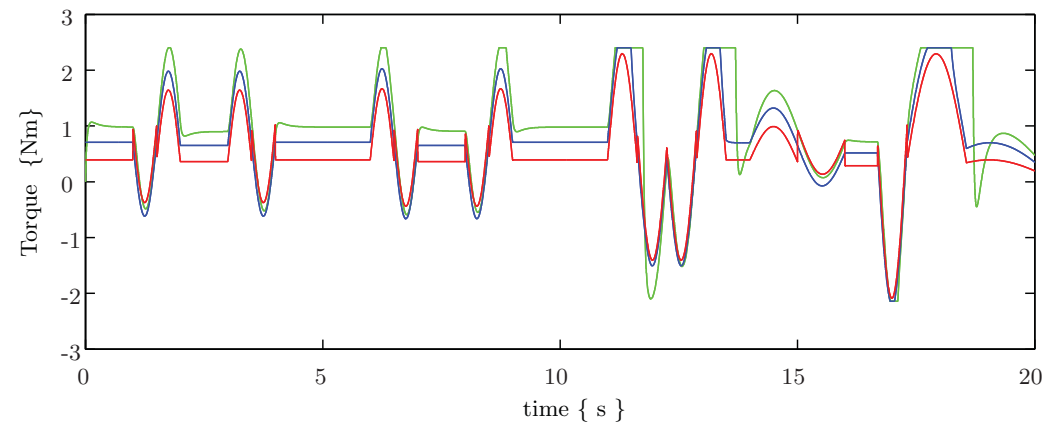

Figure 5.9: Torques applied to the pitch dynamics that correspond to Fig. 5.8a. 


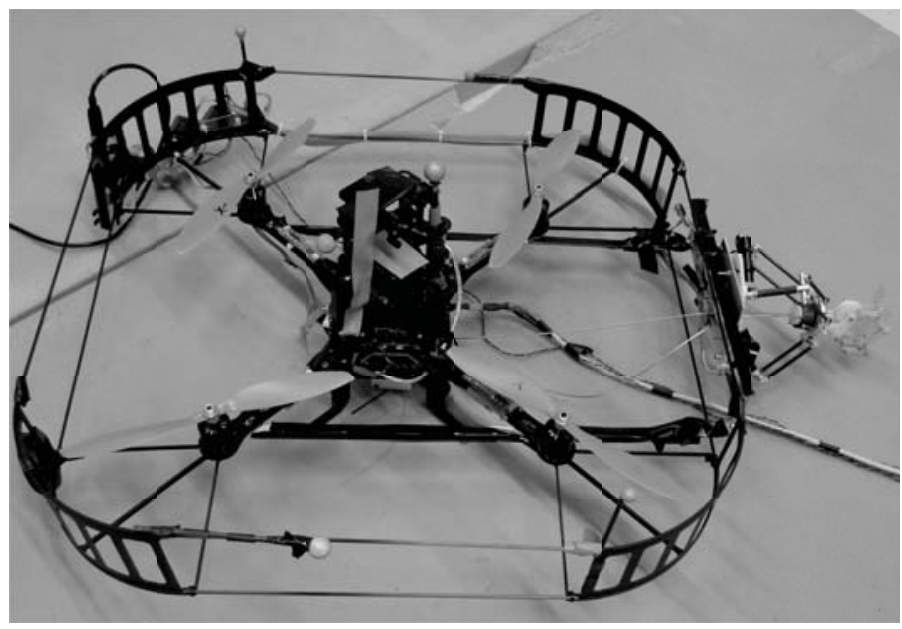

Figure 5.10: The aerial manipulator used for experimentation.

\subsection{Experiments}

The experimental setup and results that verify the claims that are made in this work are presented in this section.

\subsubsection{Experimental Setup}

The aerial manipulator used in the experiment is mainly composed of a delta manipulator [43], on which a gripper is mounted, and the Asctec pelican quadrotor, see Fig 5.10. The manipulator was designed by the University of Twente as a part of the EU AIRobots project [59] for aerial inspection by contact. It has three actuated DOFs in the Cartesian space. It is mounted on the quadrotor at $45 \mathrm{deg}$ of the $x y$ plane of $\psi^{u}$. It is equipped with a joint space position controller. For more details on the delta manipulator, interested readers are referred to [43].

On the other hand, the gripper has single DOF. It is closed and remains static during the entire experiment. It is essentially an added mass on the tip of the delta manipulator. As a result, the combined manipulator and gripper has a dominant behavior of a mass concentrated at the end-effector of the manipulator.

During the experiments, the absolute pose of the UAV-base is provided by OptiTrack (NaturalPoint, Inc. Corvallis, OR, USA) external tracking system on the basis of passive reflective markers attached on the UAV. The position of the end-effector of the manipulator, on which the mass is assumed to be concentrated at, is estimated based on joint angles obtained from the motor encoders. The control and communication software structure is mainly implemented in ROS and Matlab Simulink (MathWorks, Natick, MA, USA). 

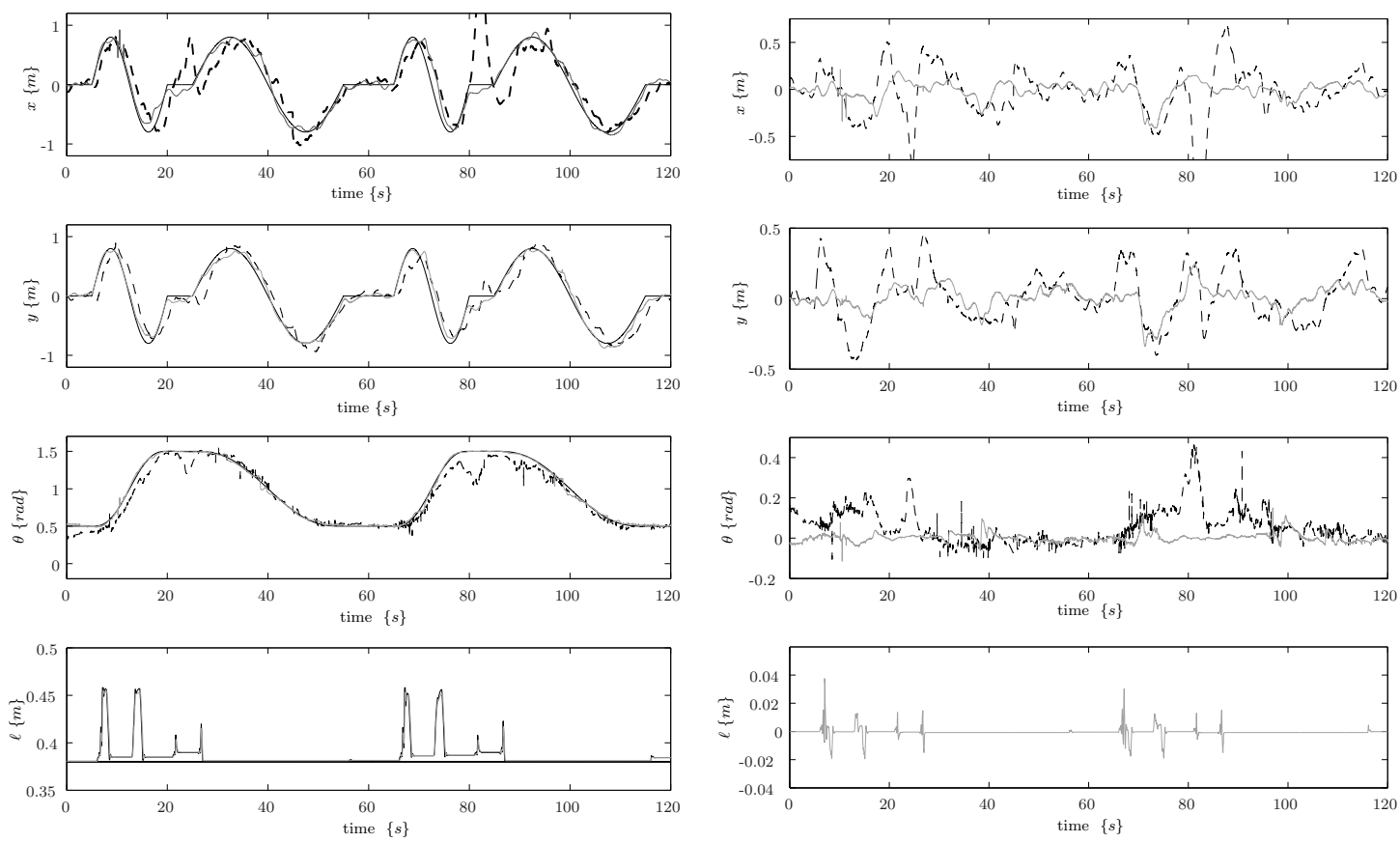

(a) States of the planar aerial manipulator.

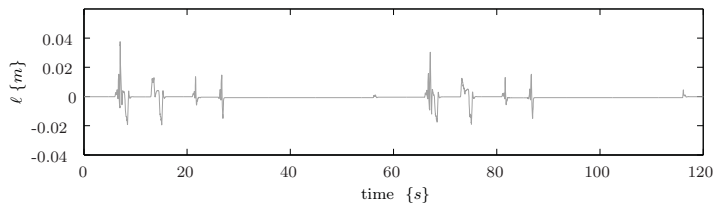

(b) Error in tracking.

Figure 5.11: Comparison of the tracking capabilities of the proposed controller (gray) and the PID controller with compensating mass (dashed). The reference is indicated by solid dark line.

\subsubsection{Experimental Results}

The experimental tests illustrate both the applicability of the proposed controller, with respect to robustness and effectiveness, and its improved performance over the state of the art. To that end, tracking performance of two different controllers in the presence of initial measurement error and system parameter uncertainties are evaluated. The implemented controller are a PID controller with compensating mass and the proposed controller that exploits the dynamics of the manipulator.

Fig. 5.11a shows the reference and real Cartesian positions of the UAV and the translational elongation of the manipulator. Fig. 5.11b shows the tracking error. Both figures clearly show that the proposed controller results in a better and satisfactory tracking performance than the PID controller with compensating mass. The slow response and poor tracking performance of the PID controller is mainly attributed to the higher inertia of and the larger gravitational effect on the aerial manipulator than the UAV alone, for which the control parameters has been originally tuned.

Remark 5.5 For fair comparison, all the controllers used both in simulations and experiments are tuned in a way that they fulfill the same control performance requirements with respect to their nominal model parameters. Besides parametric uncertainties, dynamic uncertainties that are not considered in the control design 
phase, such are aerodynamic effects, have been included in simulation and are inevitably present during experiments.

\subsection{Discussion}

The simulation and experimental results confirm that improved maneuverability, better tracking performance, and robustness can be achieved with the proposed controller. The improved maneuverability stems from the fact that its flight envelop is expanded by changing the rotational inertia and the gravitational torque, and tentatively $^{3}$ exploiting the actuation system of the manipulator. The improved performance is mainly attributed to the use of the unified dynamics of the aerial manipulator during controller design phase. As such, a feed-forward term primarily compensates for the disturbance due to the dynamics of the manipulator. The robustness is greatly improved for similar reason, which otherwise would be highly limited or even compromised due the significant constraint that the disturbance of the manipulator imposes on the stability margin of the closed loop system.

The main significance of the result is that manipulators of different type (various structures and properties) can now be designed for aerial manipulation tasks without fear of stability issues. This approach refocuses the main design requirement of the manipulator back to the task they are aspired to be used for, by essentially eliminating the constraint imposed on the mechanical design due to controller choice [43], [21]. With respect to the analysis presented in Section 5.3, the proposed controller expands the region of possible combination of $m_{m}, \ell$ and $h$ that will still keep the system dynamically stable (see Fig. 5.3) without compromising the achievable bandwidth of the system. The only constraint is imposed by the physical capability of the UAV.

In Section 5.7.2, the improved capability of the proposed controller has been shown using a manipulator, which is originally designed to perform aerial interaction without significantly influencing the dynamics of the UAV-base. Furthermore, the flight arena used during the experiment has limited workspace that do not permit very aggressive maneuvers, where the significance of the controller would be greater. As a consequence, the achieved promising results should be considered as proof of concept, not as the limit of performance that can be achieved with the controller.

From mechanical viewpoint, manipulators that have significant mass, large workspace and decoupled DOFs along the axis of the UAV can be more efficiently exploited during free-flight. Having significant mass with larger workspace increases the significance and the range of gravitational torque that can be exploited as additional actuation for the attitude dynamics. Besides, it results in a wide range of additional inertia with respect of the COM of the UAV that influences the

\footnotetext{
${ }^{3}$ The actuation system of the manipulator can only be exploited by the UAV so long as the manipulator is accelerating/decelerating. Since the manipulator has limited workspace with respect to the UAV-base, if it accelerates, then it also decelerates to come to rest. As a consequence, every positive energy supplied to the UAV during acceleration will be extracted during deceleration, or vice-verse.
} 
dynamics. Moreover, having decoupled DOFs helps in avoiding conflicting control actions along the various DOFs. Conflicting control requirements should also be handled carefully during positioning of the manipulator on the UAV, provided that such positioning does not hinder the interaction task they are originally intended for.

Remark 5.6 It should be emphasized that this work shows how to exploit the dynamics of a manipulator, primarily intended for interaction task, for significantly improving free-flight performance of UAVs.

\subsection{Conclusion}

In this chapter, a new control architecture for aerial manipulators, which is based on their unified dynamics of an aerial manipulator and exploits the additional dynamic disturbance of the manipulation system for free-flight control of the UAV-base has been presented. A clear comparison between the state of the art and the proposed controller is also provided. From performance and maneuverability points of view, the proposed controller has shown superiority over the classical UAV controllers. Moreover, systematic methods to efficiently exploit the manipulation system have been provided.

The proposed architecture relaxes the light-weight requirement and placement restrictions, and as a consequence, it extends the application spectrum that aerial manipulators can be considered. It also opens opportunities for using manipulators of different mechanical configuration and dynamic property. Furthermore, this work can be used as a basis for exploiting aerially transported loads and external interaction forces to improve the tracking or other secondary task performance of UAVs. 



\section{CHAPTER 6}

\section{Conclusions and Recommendations}

In this chapter, the main contributions of this thesis with respect to the goals, as described in Section 1.3.1, are summarized. Furthermore, recommendations for possible future research directions are provided.

\subsection{Conclusions}

The control architectures proposed in this thesis can be broadly classified into teleoperation and autonomous controllers for aerial robots and aerial manipulators. Although the slave controllers designed in the context of teleoperation for aerial robots can be used autonomously, and there are overlapping design requirements, their challenges, performance measuring metrics and ultimate goals are quite distinct. Hence, the conclusions are provided separately.

\subsubsection{Teleoperation}

The main objectives of a tele-control architecture are ensuring stability and achieving transparency to attain improved task-performance, without being hindered by the physical and/or intellectual separation of the operator and the task. To achieve those objectives in haptic teleoperation of aerial service robots, advanced control architectures were presented in this thesis. With this respect, the conclusions drawn in the first three main chapters of this thesis are recapitulated according to the main theme of each chapter.

\section{Generic and passive}

Chapter 2 of this thesis first identified peculiar challenges in the emerging field of haptic teleoperation of aerial robots, such as pervasive energy dissipation due 
to gravity and friction, underactuation of the aerial robot, and workspace incompatibility between the master haptic interface and slave aerial robot. A control architecture that addressed both the classical and peculiar challenges was proposed. This architecture is characterized by a high-level teleoperation control loop and a low-level dynamic regulation control loop. The high-level loop consists of a variable impedance controlled master system and a virtual slave that serves as a proxy for the real slave aerial robot. The employment of the virtual slave results in numerous benefits, such as, hiding the underactuation of the real slave from the human operator, and separating the energy required to maintain the flight from the energy required to maneuver the aerial robot.

A mapping strategy that bridges the gap between workspace and precision also added greatly to the flexibility of the proposed control architecture [68]. Operator initiated switching between a rate-based mapping to overcome workspace incompatibility, and a pose-based mapping to achieve precision, can occur at anytime during the course of an action, while preserving the passivity of the control loop. The fundamental principle to ensure passivity of the teleoperation control loop is to simply exchange energy-neutral-behavior-modifying reference signals across the unreliable communication channel. These signals in turn, modulate the local energy tanks of the master and the slave (primary and backup energy tanks), from which energy required to accomplish a desired task is consumed. As such, the desired behavior of the master and the slave is attained by the action of their respective controllers, provided that there is energy in their local tanks.

The clear distinction between high-level teleoperation control loop and low-level dynamic regulation control loop made the proposed control architecture generic. The high-level control loop works in a plug-and-play fashion without fear of destroying its passivity. The yearly Integration Weeks and Review Meetings of the AIRobots project were convenient platforms to test the generic nature of the proposed algorithm. Fig. 6.1 depicts snap shots taken during the Integration Weeks and Review Meetings, illustrating the applicability of the proposed algorithm with any type of haptic device, slave aerial robot, low-level controllers, and different human operators.

\section{Transparent}

The other important aspect in realization of a haptic tele-control architecture is transparency, which is related to performance. Transparency in haptic teleoperation of aerial robots was the focus of Chapter 3. Since the classical definition of transparency only considers interactive tasks with the environment, where the slave robot is a part of the tool used to manipulate the environment, in this thesis, transparency has been comprehensively defined for haptic teleoperation of aerial robots, which includes a significant free-flight regime. A time-domain definition of transparency for haptic teleoperation of mobile robots was given, in which the transparency in free-flight and interactive tasks is identified as vehicle and environment transparencies, respectively. Vehicle transparency considers the slave aerial robot as a manipulandum rather than part of the teleoperator used to manipulate 


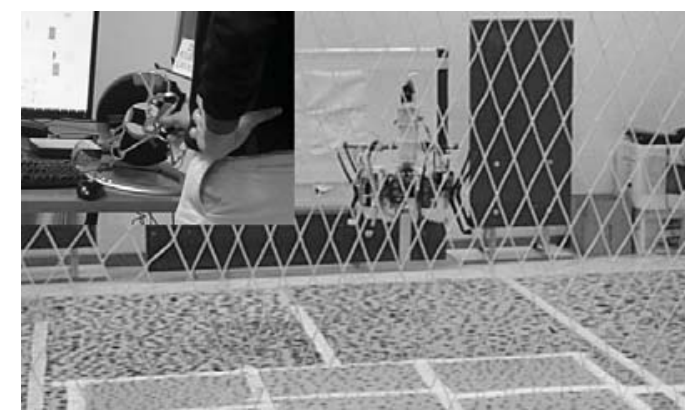

(a) The ducted-fan of the University of Bologna teleoperated using an Omega6 haptic interface.

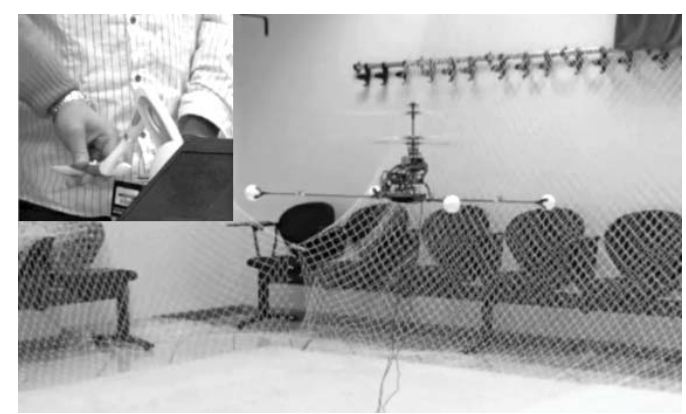

(b) A Coaxial rotor-carft from ETH-Züich teleoperated using a Phantom Omni haptic interface.

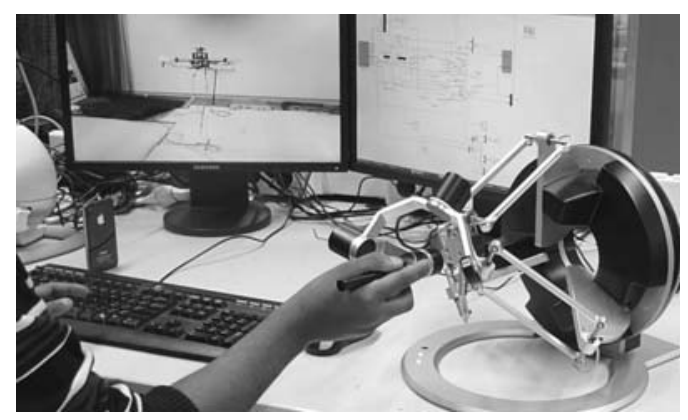

(c) An Asctect pelican quadrotor teleoperated using an Omega6 haptic interface.

Figure 6.1: Illustrations of the applicability of the control architecture proposed in Chapter 2 with different parts developed by the consortium of the AIRobots projects, such as slave aerial robots with different mechanical configurations, and their low-level controllers.

the environment, as in the classical definition of transparency. Moreover, mathematical definitions of ideal transparency and measure of transparency deviations were provided.

With aim of achieving improved transparency in the presence of networkinduced imperfections, extensions of the control architecture presented in Chapter 2 were proposed. The control architecture was realized for the commonly used admittance and impedance tele-control architectures. Different variants of the control architecture were evaluated with respect to their achievable transparencies. Moreover, transparency enhancing methodologies, such as impedance reflection and dynamic kinesthetic boundary, were implemented for both the admittance and impedance frameworks.

The control architecture was practically realized in an internet application in an intercontinental setting in the presence of significant time-varying delays and packet losses. Fig. 6.2 shows a snap shot of the longest intercontinental haptic teleoperation of aerial robots, which involved navigation of an aerial robot through a cluttered environment without colliding with obstacles. The experiment was conducted between the Australian National University, Australia, and the University of Twente, The Netherlands. 


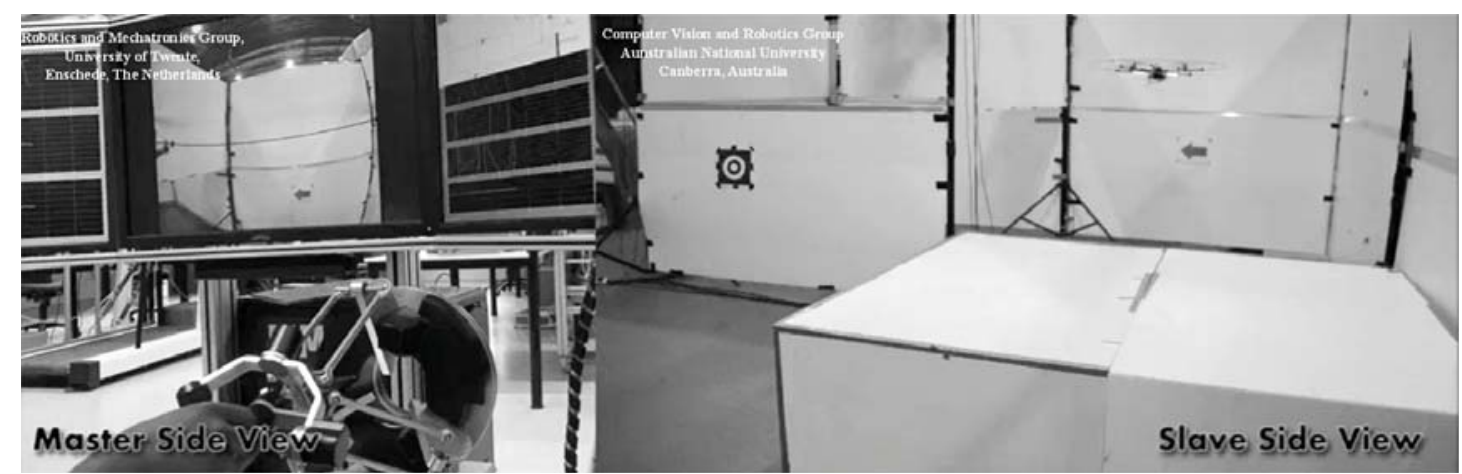

Figure 6.2: A snap shot of the longest intercontinental haptic teleoperation of aerial robots everaerial robot!VTOL UAV!quadrotor.

\section{Multimodal and versatile}

In a step towards achieving human-like manipulation capability through a telecontrol architecture, Chapter 4 proposed a versatile variable impedance control architecture for interactive aerial robots. Beyond classical teleoperation that relies on exchange of position (velocity)/force information to execute the task, the proposed controller also varies the impedance of the controlled aerial robot according to the operator impedance, which is derived by observing the operator's arm muscular activities. The designed slave controller enables stable tracking of a desired force trajectory once contact with the environment is established. The master system, on the other hand, interprets the operator's intention, which are elaborated as a desired impedance, position or force (depending on the occurrence of contact). Besides, it displays haptic feedback to achieve improved vehicle and/or environmental transparencies. Moreover, a tactile feedback closes the impedance control loop, displaying information about the current impedance of the controlled slave aerial robot and/or environment.

The ability of the controlled aerial robot to display a variable impedance enabled it to achieve higher performance in various tasks that can not be achieved with fixed impedance. In addition, the slave controller's ability to regulate a timevarying interaction force made it appealing for various tasks that require interaction with a certain level of force, while ensuring safety and desired-level of task performance. The fact that the interaction force regulation relies on desired operator force and actual interaction force, both estimated using a sliding-mode observer, highly added to the flexibility of the proposed control. Furthermore, the realization of the variable impedance controller on the master side, ensures acceptable level of transparency both in free-flight and interaction.

Simulations and experimental results demonstrated the feasibility of the proposed architecture. Its stable and transparent interaction with nonlinear environments was also illustrated. Results also showed effects of the different control parameters on the performance of the controller, providing more insight as to how the parameters need to be tuned in order to achieve a desired performance. 


\subsubsection{Autonomous}

Unlike haptic tele-control architectures, autonomous controllers enable the execution of a desired task without an energetic coupling with the human operator. The main design goals in autonomous controllers are stability and task-performance. The low-level controllers employed in Chapters 2 and 3 are trajectory tracking controllers with and without obstacle avoidance capability [80], [10], [66]. However, the slave controller in Chapter 4, and the autonomous controller presented in Chapter 5, were newly developed in light of the emerging field of aerial service robotics, which requires active interaction with the environment.

\section{Intrinsic safety and flexibility}

In the ever expanding field of service robotics, in which robots are used for manipulation of objects with different types of geometry and dynamic properties, safety has higher precedence. Hence, inclusion of safety measures during controller design is important, as in for example [81]. Acknowledging the importance of safety and due to the characteristics of impedance controllers to safely handle unexpected transition between free-flight and interaction, a variable impedance controller was designed in Chapter 4. The autonomous capability of the slave controller was demonstrated in [73]. The inner-loop variable impedance controller, the outer-loop force controller, and the sliding-mode observer enable the controlled aerial robot to track a desired time-varying interaction force without the use of force sensors while displaying a variable impedance. It was shown that the controller robustly interacts in a stable manner with both linear and nonlinear environments, including those whose properties change suddenly.

\section{Modular vs Unified}

Chapter 5 focused on the design of an autonomous free-flight controller for aerial manipulators. It revisited the current state of the art in free-flight control of aerial manipulators, which relies on modular approach. In the modular approach, the controllers of the aerial robot and the robotic manipulator are designed independently. In particular, the aerial robot controller is designed to robustly reject both static and dynamic disturbances induced by the robotic manipulator. However, the analysis given in this chapter showed that the robustness is achieved at the expense of performance, which may also result in some stability issues. More importantly, such approach also imposes significant limitations on the dynamic properties of the robotic manipulator that can be appended to the aerial robot.

The new controller presented in the chapter was designed based on the unified dynamics of the aerial manipulator. As a result, the stability robustness of the controlled aerial manipulator and its performance improved greatly. Furthermore, the unified approach essential removes the constraints imposed on the properties on robotic manipulators that can be appended due to the choice of a controller designed based on the modular approach. It was also shown that by further exploiting the dynamics of the robotic manipulator during free-flight, it was possible to even 
expand the flight envelop, with respect to its other counterparts. It was concluded that with the proposed framework, a number of manipulators with wide range of dynamics and applications can be employed, while their dynamics is exploited to achieve better performance in control of UAVs during free-flight.

\subsubsection{Overall Conclusion}

In this thesis, advanced teleoperation and autonomous control architectures for aerial service robots were proposed. In particular, teleoperation controllers for aerial robots and autonomous free-flight controller for aerial manipulators were presented. The teleoperation algorithms in the first three main chapters of this thesis can be directly applied to aerial manipulators by modifying the point of interest to the end-effector of the aerial manipulator.

The feasibility of the control architectures and their effectiveness were demonstrated by extensive simulations and experiments that involve realistic scenarios, beyond just proof-of-concept. Practical considerations that are necessary for effective utilization of the proposed architectures were also provided. Moreover, control parameter tuning methodologies were discussed by illustrating their effect on stability and task-performance. Although the proposed control architectures were tailored for aerial service robots, they can be applied without or with minor modification to other fields of robotics.

\subsection{Recommendations}

Even if this thesis addressed numerous research questions that arose in the field of aerial service robotics, there are still many more open questions. Some of the open questions, in the context of this research, are recommended for future research.

\section{Towards industrial applications}

The proposed algorithms were experimentally tested extensively in realistic scenarios. However, they were conducted in indoor laboratory environments, which were equipped with external positioning systems, and where external disturbances were limited, such as wind gust. Although experiments were conducted in the AIRobots' mockup environments, not all real-time challenges were present. Hence, as a step towards industrial applications, the control architectures need to be tested in outdoor as well as GPS denied environments. This requires measurement data from on-board sensors and data fusion to estimate the states of the aerial robots, which in turn needs sensors and high-capacity computational boards that would impose significant constraint on the energy autonomy of the aerial service robot due to their heavy payloads. Hence, future works need to address safety, robustness to environmental disturbance, reliable and robust data fusion from on-board exteroceptive and proprioceptive sensors, and energy autonomy, cohesively. 


\section{Shared and flexible human-robot interaction}

In the tele-control architectures presented in this thesis, the human operator played a supervisory role. The operator defined and continually monitored the task, while the controlled aerial robot enjoyed tracking, and obstacle avoidance autonomy. However, emerging and next generation applications require strong cooperation between the operator and the robot. This cooperation needs strong symbiotic relation between the two, and possession of autonomy. Successful cooperation between the human and robot can be exploited in teleoperation settings as well. But, such cooperation goes beyond just interaction through position/force. As a step forward in this direction, multimodal interaction between the operator and the slave was realized in the control architecture presented in Chapter 4. Nevertheless, establishing full-fledged cooperation requires understanding of the intentions (including emotional states) of the operator and the robot, multimodal communication between the two, and robust autonomy. To that end, inclusion of other ways of communication and various level of autonomy that may evolve during the course of action need to be investigated.

\section{Psycho-physical analysis}

Although through extensive experimental results, the applicability of the proposed tele-algorithms without significant mental overload was observed, it is important to conduct psycho-physical analysis. Detailed psycho-physical evaluation on the merit of each algorithm, especially the architecture presented in Chapter 4, with respect to operator convenience and task performance, needs to be investigated. This will definitely provide more insight on how to better exploit the proposed algorithms.

\section{Additional recommendations}

- It was observed that network-induced imperfections impose significant trait on stability and performance of the proposed teleoperation algorithms. Despite the fact that the proposed tele-control architectures essentially neutralized the trait on stability, the effect on performance is still not fully overcome. In this direction, it is recommended to exploit the information about the amount of time-varying delays and packet losses in the network to improve reliability of the signals exchanged, and to further use this information in predictive tele-control architectures.

- In addition to the spring and damper, it is beneficial to consider modulation of the apparent inertia of the controlled aerial robot in the control architecture proposed in Chapter 4, mainly to limit impact forces during aerial interaction. Moreover, employment of a 3D contact dynamics in the architecture will improve the performance of simultaneous interaction force regulation and sliding on a surface. Furthermore, future works should consider extension of the proposed control algorithm to accommodate multiple interaction points, and robust online identification of environmental impedance parameters. 
- The free-flight control architecture presented in Chapter 5 can be further extended in order to exploit the dynamics of the load transported by the manipulator. It is worth applying an adaptive control paradigm to identify the inertia property of the grasped object, and exploit the combined dynamics of the manipulator and the load accordingly. 
Appendices 



\section{APPENDIX A}

\section{Modeling and Control of underactuated AERIAL RoBots}

This appendix presents a generic model and a controller design approach for a class of underactuated aerial vehicles, namely for unmanned aerial vehicles whose primary support against gravity is thrust. The approach followed is based on energetic consideration and uses the formalisms of port-Hamiltonian systems and bond graphs. The controller is designed for both setpoint stabilization and trajectory tracking tasks. The competency of the model and the performance of the controller are validated in simulation.

This appendix is an adapted version of (Mersha et, al, 2011); Mersha, A.Y., Carloni, R., and Stramigioli, S., "Port-based Modeling and Control of Underactuated Aerial Vehicles", In Proceedings of the IEEE International Conference on Robotics and Automation, 2011. 


\section{A.1 Introduction}

Miniaturized UAVs are in general characterized by underactuatedness, highly nonlinear and inherently coupled dynamics, which often make the design of autonomous controller challenging and, at the same time, interesting research problem. While earlier controller design attempts are based on linear control techniques, in recent years, the trend has shifted to designing nonlinear control laws to enhance performance and improve controllability in a wide range of the flight envelope [80], [37]. However, most of the controllers designed as of now are aeromechanical platform specific.

Generally, closer look at the actuation strategies of common UAVs, whose primary support against gravity is thrust, such as VTOL UAVs, reveals their similarity. They are often characterized by fully actuated rotational and underactuated translational dynamics. Utilizing this fact and making few reasonable assumptions near hovering, i.e, low velocity flights, their dynamics can be simplified and converge to having similar form irrespective of their type and mechanism of actuation. This can be utilized to the benefit of devising a general control strategy for this class of flying vehicles.

In this appendix, a generic model and controller design for both setpoint stabilization and trajectory tracking by using the port-based framework is presented. The motivation behind using such an approach is that it enables treating controllers as dynamical systems, which exchange energy with the plant with an ultimate goal of influencing the dynamics of the plant to behave as desired. This approach, which is quite different from the classical method of treating controllers as signal processors, also enables to incorporate information about the structure of the physical system for ease of designing the controller and tuning its parameters with more intuitive insight [86].

The contribution of this appendix, other than enriching existing literature on the port-Hamiltonian framework, lies in providing a unified model and an elegant controller design approach for the class of VTOL UAVs.

\section{A.2 Dynamic Model}

In this Section, the dynamic model of a generic VTOL UAV and its bond graph representation are presented.

\section{A.2.1 Equation of Motion}

The equation of motion of UAVs is often described by means of two coordinate frames, i.e., $\psi^{0}$ and $\psi^{b}$, which represent the inertial and the body fixed coordinate frames, respectively. The choice of $\psi^{b}$ at the center of gravity, aligned with the principal axis, simplifies the inertial tensor to a diagonal matrix, thereby reducing computational load. 
The dynamic equation of a generic UAV can be derived by considering it as a rigid body in the special Euclidean configuration space $S E(3)=\left\{S O(3) \times \mathbb{R}^{3}\right\}$, where $S O(3)$ is the Lie group indicating the set of orthonormal rotational matrices. Various wrenches act on this rigid body, such as thrust, gravity, lift, drag, antitorque, and gyroscopic precession.

Considering the gravitational attraction force as an external wrench applied to a rigid body rather than stored potential energy, the equation of motion of a generic UAV expressed in $\psi^{b}$ in port-Hamiltonian form is given by

$$
\left\{\begin{array}{l}
\dot{P}_{b}^{b}=\widetilde{P}_{b}^{b} \frac{\partial H\left(P_{b}^{b}\right)}{\partial P_{b}^{b}}+W_{b}^{b} \\
T_{b}^{b, 0}=\frac{\partial H\left(P_{b}^{b}\right)}{\partial P_{b}^{b}}
\end{array}\right.
$$

where the generalized momentum $P_{b}^{b}=I_{b}^{b} T_{b}^{b, 0}$ and twist $T_{b}^{b, 0}$ are the state and the passive output of the UAV, respectively. Moreover; the energy of the system is described by the Hamiltonian function $H\left(P_{b}^{b}\right)=\frac{1}{2}\left(P_{b}^{b}\right)^{T}\left(I_{b}^{b}\right)^{-1} P_{b}^{b}, \widetilde{P}_{b}^{b}=$ $\left(\begin{array}{cc}\widetilde{P}_{b-\omega}^{b} & \widetilde{P}_{b-v}^{b} \\ \widetilde{P}_{b-v}^{b} & 0\end{array}\right)$, in which $\widetilde{P}_{b-\omega}^{b}$ and $\widetilde{P}_{b-v}^{b}$ are skew symmetric matrices composed of the first and the last three elements of $P_{b}^{b} . W_{b}^{b}$ collects all wrenches acting on the UAV, including those due to the control action.

It is common to describe the generalized configuration of flying vehicles in a six dimensional vector $q$, consisting of Euler angle representation of the orientation (the roll $\phi$, the pitch $\vartheta$ and the yaw $\psi$ with respect to $x, y, z$ axes, respectively) and the position $(x, y, z)$. The kinematic relation between $T_{b}^{b, 0}$ and $\dot{q}$ is given by $\dot{q}=Z T_{b}^{b, 0}$, where $\mathrm{Z}$ is a $6 \times 6$ transformation matrix given by $Z=\left(\begin{array}{cc}G & 0 \\ 0 & R_{b}^{0}\end{array}\right)$, in which, $R_{b}^{0}$ and $G$ are $3 \times 3$ rotational and transformation matrices, respectively. $G$ transforms angular velocity of a body to rate of change of Euler angles.

\section{A.2.2 Bond Graph Representation}

A simplified bond graph representation of a generic UAV is given in Fig. A.1a. In particular, the UAV is composed of a rigid body on which various wrenches, collected by $W_{b}^{b}$, act. In the bond graph, $\mathbb{M} \mathbb{G} \mathbb{Y}$-element represents the gyration term, and $\mathbb{I}$ the inertial tensor of the UAV, which is characterized by the internal energy of the system described by the energy function $H\left(P_{b}^{b}\right)$. The constitutive equation at the 1 -junction, representing $T_{b}^{b, 0}$, results in (A.1).

For better insight, especially during the controller design, wrenches collected by $W_{b}^{b}$ can be further split into: gravity wrench $W_{\text {grav }}^{g}$, expressed in $\psi^{g}$ which has the same orientation as $\psi^{0}$ but fixed at the center of gravity; the wrench $W_{\text {cont }}^{b}$ due to control action, and other wrenches acting on the UAV expressed in $\psi^{0}$ and $\psi^{b}$, collected by $W_{b}^{0}$ and $W_{b 1}^{b}$, respectively (see Fig. A.1b). The two $\mathbb{M T T F}$ elements in the figure represent coordinate transformation. It can be noted that the UAV model is power continuous.

Due to the modular nature of the port-Hamiltonian framework and the bond graph representation, detailed modeling of other physical domains contributing to 


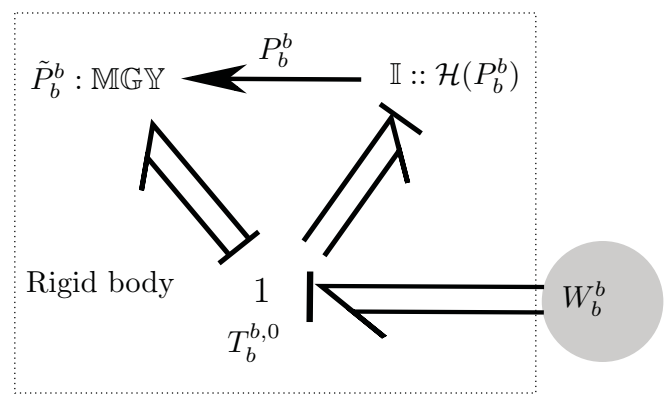

(a) UAV model

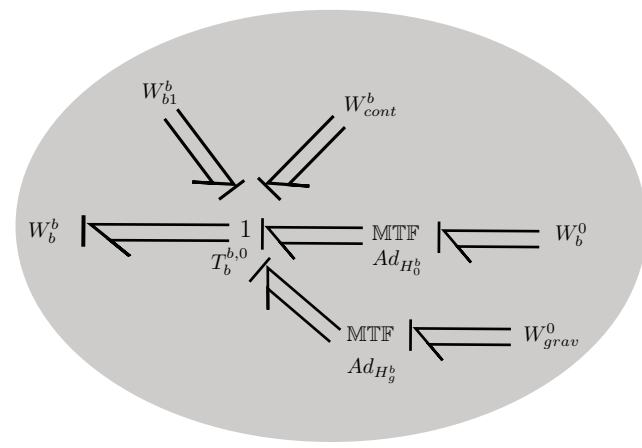

(b) Extended model of $W_{b}^{b}$

Figure A.1: Bond graph representation a generic UAV model - (a) The power continuous Dirac structure of the UAV is realized by interconnecting a modulated gyrator, i.e. a $\mathbb{M} \mathbb{G} Y$-element, a 1-junction, an inertial element $\mathbb{I}$ and external port $(u, y)=\left(-W_{b}^{b}, T_{b}^{b, 0}\right)$. (b) The generalized wrench $W_{b}^{b}$, acting on the UAV, is given by the sum of different wrenches. Note that $\mathbb{M} \mathbb{G} \mathbb{Y}$ and $\mathbb{M T F}$ are power conserving elements.

the dynamic behavior of the UAV can be added by appropriate interconnection. However, unless detailed study on the dynamic behavior of each part of the UAV or optimization during design phase is required, the model represented in Fig. A.1a is adequate for controller design.

\section{A.3 Controller Design}

In this Section, a generic controller is designed in port Hamiltonian formalism for hovering and trajectory tracking tasks. The controller design strategy is based on treating controllers as dynamical systems exchanging energy with the plant, i.e, the UAV. This approach enables to recast controller design as finding dynamic system and its interconnection with the system so as to influence its dynamic behavior as desired [86].

The control problem in UAVs is to design a control law that enables the stabilization and/or the tracking of the trajectories of $x, y, z$ and $\psi$ by using four control inputs such as thrust, AOA, swash plate angles, which are platform specific. To keep the controller design methodology generic, specific control inputs are not considered at this point. Rather, the desired effective wrench on the vehicle as a consequence of the control action is considered. To simplify the discussion, first a controller for hovering tasks is presented.

\section{A.3.1 Stabilization Controller}

Stabilization or hovering is a set point regulation problem. The desired equilibrium states (Configuration $q$ and Momentum $P$ ) of the UAV are of the form $(q, P)=$ $(\bar{q}, 0)$, where $\bar{q}$ is the desired configuration. This problem, in the approach followed 
here, can be recast as finding a dynamic system and proper interconnection that transforms the controlled plant to a passive system by shaping its original energy in such a way that the energy function of the complete system has a global and unique minimum at the desired configuration. For mechanical systems, it is adequate to shape their potential energy since their allowable equilibrium states naturally correspond to the minimum value of the kinetic energy.

Consider a controller with a potential energy of the form $H(q)=\frac{1}{2}(q-\bar{q})^{T} K_{p}(q-$ $\bar{q})$, which attains its minimum at the desired configuration $\bar{q}$ for some positive definite matrix $K_{p}$. By using (A.1), the controlled port-Hamiltonian system, with total energy function $H(q, P)=\frac{1}{2}(q-\bar{q})^{T} K_{p}(q-\bar{q})+\frac{1}{2}\left(P_{b}^{b}\right)^{T}\left(I_{b}^{b}\right)^{-1} P_{b}^{b}$, becomes

$$
\left\{\begin{aligned}
\left(\begin{array}{c}
\dot{q} \\
\dot{P}
\end{array}\right) & =\left(\begin{array}{cc}
0 & Z \\
-Z^{T} & \widetilde{P}_{b}^{b}
\end{array}\right)\left(\begin{array}{c}
\frac{\partial H(q, P)}{\partial q} \\
\frac{\partial H(q, P)}{\partial P}
\end{array}\right)+\left(\begin{array}{c}
0 \\
I
\end{array}\right) u^{\prime} \\
y & =\left(\begin{array}{ll}
0 & I
\end{array}\right)\left(\begin{array}{c}
\frac{\partial H(q, P)}{\partial q} \\
\frac{\partial H(q, P)}{\partial P}
\end{array}\right)
\end{aligned}\right.
$$

By comparing (A.2) of the controlled system with the autonomous port-Hamiltonian system given by (A.1), it is possible to find the relations

$$
u^{\prime}=W_{b}^{b}+Z^{T} K_{p}(q-\bar{q}) \text { and } y=T_{b}^{b, 0}
$$

The controlled port-Hamiltonian system is lossless and can be stabilized at the desired equilibrium state $\bar{q}$ by simply employing a dissipative control law of the form $u^{\prime}=-K_{d} y=-K_{d} T_{b}^{b, 0}$, for some positive definite matrix $K_{d}$. Now, back tracking to find the original control law yields

$$
W_{b}^{b}=-Z^{T} K_{p}(q-\bar{q})-K_{d} T_{b}^{b, 0}
$$

which is physically interpreted as a combined action of a spring (with a constant $K_{p}$ ) and a damper (with a constant $K_{d}$ ). Both $K_{p}$ and $K_{d}$ are $6 \times 6$ matrices.

In bond graph, the reshaping of the original energy can be realized by adding a storage element $\mathbb{C}$, which stores potential energy of the desired form, whereas damping injection is realized by connecting an $\mathbb{R}$ element to $T_{b}^{b, 0}$. The constitutive equation of efforts at the 1-junction representing $T_{b}^{b, 0}$ in Fig. A.2a results in (A.3).

The desired resultant behavior of $W_{b}^{b}$ given in (A.3) and shown in Fig. A.2a requires $W_{\text {cont }}^{b}$, the wrench due to controller action, to provide the desired springdamper action and compensate for other wrenches acting on the UAV, represented by $W_{\text {comp }}^{b}$ (see Fig. A.2b). It follows that

$$
W_{\text {cont }}^{b}=W_{b}^{b}-W_{\text {comp }}^{b}
$$

which gives the desired behavior of the wrench acting on the UAV due to the control action to hover around the desired set point. Note that since the UAV is underactuated, the maximum degree of freedom one can have control over is equal to the number of independent control inputs, often four. Since in most cases the DOFs one wants to have control over are $x, y, z$ and $\psi$, references of $\phi$ and $\vartheta$ are constrained by the demand on the desired set points of the rest. These references are computed from (A.4) but are platform dependent, see Section A.4. 


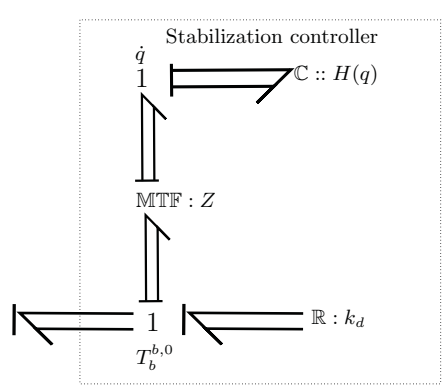

(a) Desired $W_{b}^{b}$

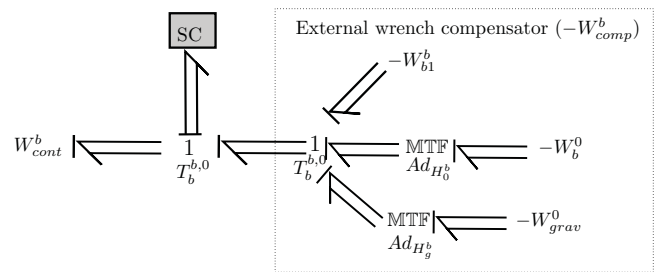

(b) Desired $W_{\text {cont }}^{b}$

Figure A.2: Bond graph representation of stabilization controller (SC) - (a) The desired behavior of $W_{b}^{b}$ is given by a spring $\mathbb{C}$ and a damper $\mathbb{R}$. (b) $W_{\text {cont }}^{b}$ is the desired wrench due to the actual controller actions. To result in the required behavior of $W_{b}^{b}, W_{\text {cont }}^{b}$ need to provide both the spring-damper effect (Block $S C$ ) and compensate for other external wrenches $W_{\text {comp }}^{b}$. The content of the SC Block in (b) is the one shown in (a).

It is very crucial to notice that the control over the translational DOFs, which are not directly actuated, comes at the expense of the rotational DOFs whose references are constrained. Hence, it is clear that the desired forces of the control wrench are obtained by the coordinated control of the orientation of the UAV along $x, y$ and the thrust. This indirect control of the underactuted translational dynamics through the fully actuated rotational dynamics results in a cascaded structure.

The final step in the controller design is to map the desired $W_{\text {cont }}^{b}$ onto the actual controller inputs represented by a four dimensional vector $\zeta$. For ease of mapping, $W_{\text {comp }}^{b}$ is approximated by collecting wrenches of less relevance, such as small body forces and unknown disturbances, in $\Delta W_{\text {comp }}^{b}$. This term is considered as perturbation and is neglected during mapping. It follows that

$$
\begin{gathered}
W_{\text {comp }}^{b}=W_{\mathrm{comp}_{\mathrm{n}}}^{b}+\Delta W_{\mathrm{comp}}^{b} \\
\Rightarrow W_{\mathrm{cont}}^{b} \approx W_{b}^{b}-W_{\mathrm{comp}_{\mathrm{n}}}^{b}
\end{gathered}
$$

To conclude the stabilization controller design, the mapping of $W_{\text {cont }}^{b}$ to actual platform specific actual control inputs $\zeta$ such as thrust, AOA and swash plate angle can be generalized by a function $\varrho(\cdot)$

$$
\zeta=\varrho\left(W_{\text {cont }}^{b}\right)
$$

\section{A.3.2 Trajectory Tracking Controller}

The main strategy proposed to achieve trajectory tracking is stabilizing the portHamiltonian systems of the error dynamics of the UAV. By doing so, the control technique proposed in the previous subsection can be employed to stabilize the vehicle to the desired trajectory.

Unlike hovering, only reshaping of potential energy is not enough for tracking problems. This is apparent since the desired equilibrium states are of the 
form $(q, P)=(\bar{q}, \bar{P})$. Therefore, the kinetic energy of the system also needs to be reshaped to address this problem. Define the error in the configuration as $q_{e}(t)=q(t)-\bar{q}(t)$, and the momentum error as $P_{e}(t)=P(t)-\bar{P}(t)=I_{b}^{b}\left(T_{b}^{b, 0}-\right.$ $\left.\bar{T}_{b}^{b, 0}\right)=I_{b}^{b} T_{e}^{b, 0}$. The error dynamics of the system, whose Hamiltonian function is $H\left(q_{e}, P_{e}\right)=\frac{1}{2} q_{e}^{T} K_{p} q_{e}+\frac{1}{2} P_{e}^{T}\left(I_{b}^{b}\right)^{-1} P_{e}$ with $K_{p}$ a positive definite matrix, is given in port-Hamiltonian form by

$$
\left\{\begin{aligned}
\left(\begin{array}{c}
\dot{q}_{e} \\
\dot{P}_{e}
\end{array}\right) & =\left(\begin{array}{cc}
0 & Z \\
-Z^{T} & \widetilde{P}_{b}^{b}
\end{array}\right)\left(\begin{array}{c}
\frac{\partial H\left(q_{e}, P_{e}\right)}{\partial q_{e}} \\
\frac{\partial H\left(q_{e}, P_{e}\right)}{\partial P_{e}}
\end{array}\right)+\left(\begin{array}{c}
0 \\
I
\end{array}\right) u_{e} \\
y_{e} & =\left(\begin{array}{ll}
0 & I
\end{array}\right)\left(\begin{array}{c}
\frac{\partial H\left(q_{e}, P_{e}\right)}{\partial q_{e}} \\
\frac{\partial H\left(q_{e}, P_{e}\right)}{\partial P_{e}}
\end{array}\right)=T_{e}^{b, 0}
\end{aligned}\right.
$$

Based on the fact that the natural equilibrium states of the above lossless controlled port-Hamiltonian system fulfills the desired trajectory tracking goal, i.e, $\left(q_{e}(t), P_{e}(t)\right)=(0,0) \Leftrightarrow q(t)=\bar{q}(t)$ and $P(t)=\bar{P}(t)$, it can be asymptotically stabilized by damping injection, i.e., $u_{e}=-K_{d} y_{e}=-K_{d} T_{e}^{b, 0}$, for any positive definite matrix $K_{d}$. Following similar line of reasoning and procedure as in the stabilization case, $W_{b}^{b}$ for trajectory tracking is

$$
W_{b}^{b}=\dot{\bar{P}}-\widetilde{P}_{b}^{b} \bar{T}_{b}^{b, 0}-Z^{T} K_{p} q_{e}-K_{d} T_{e}^{b, 0}
$$

and $W_{\text {cont }}^{b}$ is computed by using (A.5). Finally, this desired wrench behavior can be mapped into actual control inputs that are platform specific as in the stabilization case by using (A.6). The bond graph representation of the proposed trajectory tracking controller is shown in Fig. A.3.

Note that stabilization is a special case of trajectory tracking in which the set points $\bar{q}$ are fixed points, thereby resulting in a zero valued $\dot{\bar{q}}$ and $\bar{P}$. Simplification of (A.8) during a setpoint stabilization results in (A.3).

\section{A.3.3 Robustification}

The performance of the tracking controller designed in the previous section degrades as the discrepancy between the actual UAV and its model used in the controller design increases. So, to robustify the controller, an integral action on the configuration error $q_{e}$ is appended to the current control laws given in (A.3) and (A.8). To limit the integral action to a certain level in order to avoid unnecessary build up, deterioration of performance and sometimes instability as a consequence, a nonlinear integral action of the form given in (A.9) is used.

$$
\lambda\left(q_{e}\right)= \begin{cases}\bar{\lambda} & q_{e}(t)>\bar{q}_{e} \\ K_{I} \int q_{e}(v) d v & \bar{q}_{e} \leq q_{e}(t) \leq \underline{q}_{e} \\ \underline{\lambda} & q_{e}(t)<\underline{q}_{e}\end{cases}
$$

where $\bar{\lambda}$ and $\underline{\lambda}$ are the maximum and minimum allowable integral action respectively. $K_{I}$ is a $6 \times 6$ constant matrix.

It follows that, using (A.5), (A.8) and (A.9), the complete desired behavior of $W_{\text {cont }}^{b}$ becomes 


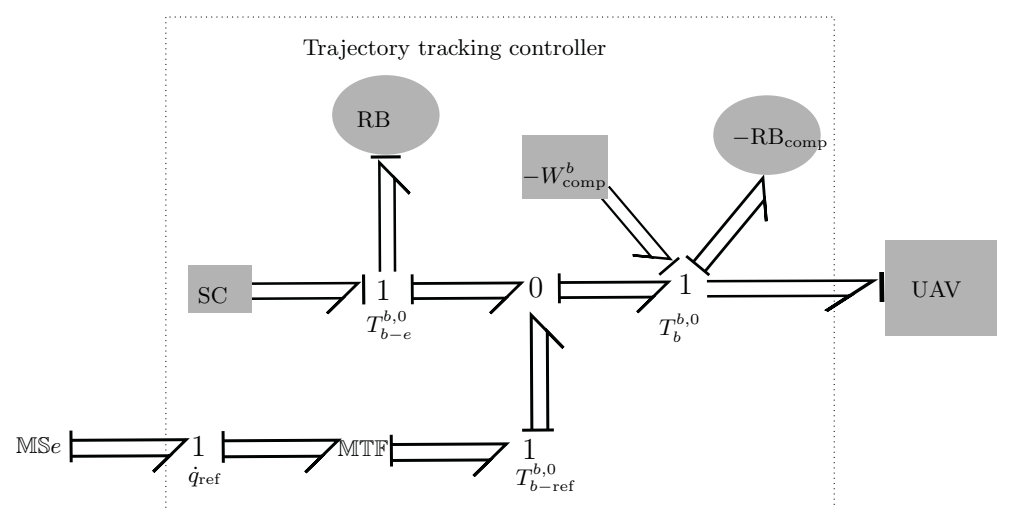

Figure A.3: Bond graph representation of the trajectory tracking controller. The block $-W_{\text {comp }}^{b}$ compensates for the external wrenches acting on the UAV. The $R B_{\text {comp }}$ block is a rigid body representation to compensate for the UAV's dynamics. To preserve the original structure, a target rigid body $R B$ is run by the error dynamics. This is then stabilized by a spring-damper action as in the stabilization case. The actual desired wrench that act onto the UAV is obtained by computing the effort on the bond entering the UAV. It gives (A.8).

$$
W_{\text {cont }}^{b}=\dot{\bar{P}}-\widetilde{P}_{b}^{b} \bar{T}_{b}^{b, 0}-Z K_{p} q_{e}-K_{d} T_{e}^{b, 0}-\lambda\left(q_{e}\right)-W_{\text {comp }}^{b}
$$

Note that in (A.10), had it not been for the integral action introduced to quell uncertainties, the parameters $K_{p}$ and $K_{d}$ which are associated to spring and damper, could have been tuned from physical considerations. But, during the presence of integral action, a tuning method from linear control analysis can be used, $[65]$.

\section{A.4 Results}

In this Section, simulation results illustrating the performance of the proposed controllers are given with an application example using a Ducted Fan Miniaturized Aerial Vehicle (DFMAV). First, a brief description of the DFMAV is provided, [80]. Then, the generic controller designed in the previous sections is systematically adapt to this platform by specifying the mapping of desired wrench to actual control inputs. Finally, representative simulation results are given.

\section{A.4.1 Brief Description of the Ducted Fan UAV}

The DFMAV is an aeromechanical platform with simple mechanics and it has intrinsically safe propeller, which is enclosed in a shroud. It also has a good thrust generation capability due to the presence of an annular body part [80]. The DFMAV is a thrust propelled UAV in which the vanes, immersed in the down wash of the main propeller, are responsible for controlling the orientation of the UAV. The DFMAV contains three sets of independent vanes, whose AOA (denoted $a, b$, c) are controlled by servo motors. 
Similar to other UAVs, the dynamic equation of the DFMAV is given by (A.1), but the wrench $W_{b}^{b}$ acting on it is platform and operating condition specific. The wrench $W_{b}^{b}=\left(\tau^{b} f^{b}\right)^{T}$ near hover condition is given by

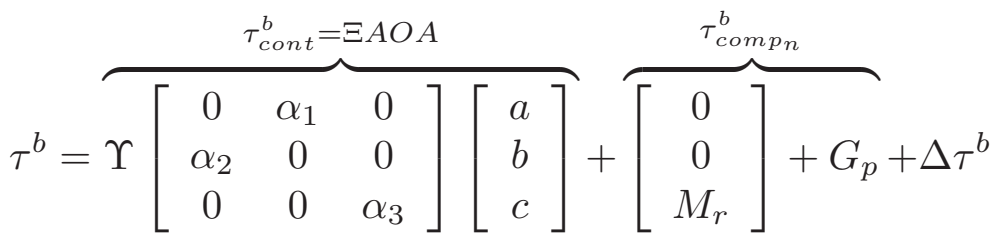

$$
\begin{aligned}
& f^{b}=\overbrace{\left[\begin{array}{c}
0 \\
0 \\
\Upsilon
\end{array}\right]}^{F_{\text {cont }}^{b}}-\overbrace{R_{0}^{b}\left(\left[\begin{array}{c}
0 \\
0 \\
m g
\end{array}\right]-\varkappa\left[\begin{array}{c}
\dot{x} \\
\dot{y} \\
0
\end{array}\right]\right)}^{F_{\text {comp }}^{b}}+\Delta f^{b}
\end{aligned}
$$

where $M_{r}=\eta \Upsilon$ is the reaction torque, $G_{p}$ is the gyroscopic precision due to the propeller, $\Upsilon$ is the thrust, $g$ is the gravity and $\varkappa$ is a constant in the momentum drag and $\alpha_{i}$ for $i=1,2,3$ are also constants. Other wrenches, including disturbances, are collected by $\Delta$ and are neglected during the control design. For detailed treatment and associated formulae, readers are referred to [65].

The control problem for the DFMAV can be restated as designing four control inputs $\zeta=(\Upsilon, a, b, c)$ such that the UAV is able to track the desired trajectories of $\bar{x}, \bar{y}, \bar{z}, \bar{\psi}$. First, the wrench acting on the vehicle is categorized into relevant and negligible as in (A.4) and (A.5) (see (A.11) and (A.12)). Then, the desired behavior of $W_{\text {cont }}^{b}$ given in (A.10), along with A.12), is used to compute the desired roll and pitch references as

$$
\left(\begin{array}{c}
\bar{\phi} \\
\bar{\vartheta}
\end{array}\right)=\left(\begin{array}{c}
\arctan \left(\frac{c_{\bar{\vartheta}} s_{\bar{\phi}} W_{\text {cont }}^{0}[4]-c_{\bar{\vartheta}} c_{\bar{\phi}} W_{\text {cont }}^{0}[5]}{W_{\text {cont }}^{0}[6]}\right) \\
\arctan \left(\frac{c_{\bar{\phi}} W_{\text {cont }}^{0}[4]+s_{\bar{\phi}} W_{\text {cont }}^{0}[5]}{W_{\text {cont }}^{0}[6]}\right)
\end{array}\right)
$$

To avoid overturning and artificial singularity due to using Euler angles, additional constraint on the reference orientation is imposed by $\|\bar{\phi}(t)\|_{\infty}<\frac{\pi}{2}$ and $\|\bar{\vartheta}(t)\|_{\infty}<$ $\frac{\pi}{2}$.

Similarly, the reference thrust is given by

$$
\bar{\Upsilon}=\frac{W_{c o n t}^{0}[6]}{c_{\bar{\phi}} c_{\bar{\vartheta}}}
$$

Note that unique extraction of $\bar{\Upsilon}, \bar{\phi}$ and $\bar{\vartheta}$ are only possible as long as $W_{b}^{0}[4: 6]$ obtained from (A.12) is not a zero vector and $W_{b}^{0}[6]>-m g$. Finally, from (A.10) and (A.12), the desired AOA is computed as

$$
A O A_{\text {ref }}=\Xi^{-1}\left(W_{\text {cont }}^{b}\right)[1: 3]
$$

(A.14) and (A.15) represent the mapping of $W_{\text {cont }}^{b}$ to actual controller inputs $\zeta$ given in (A.6) in its generic form. 


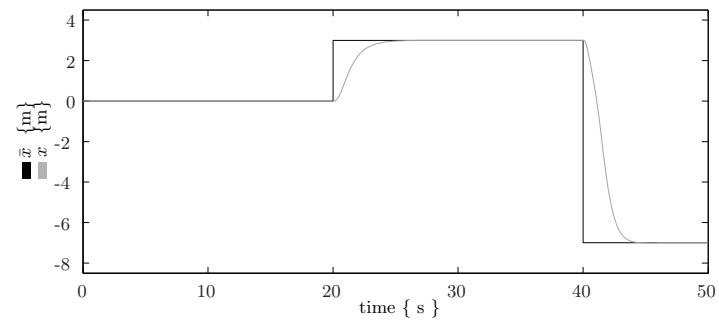

(a) $x$-position

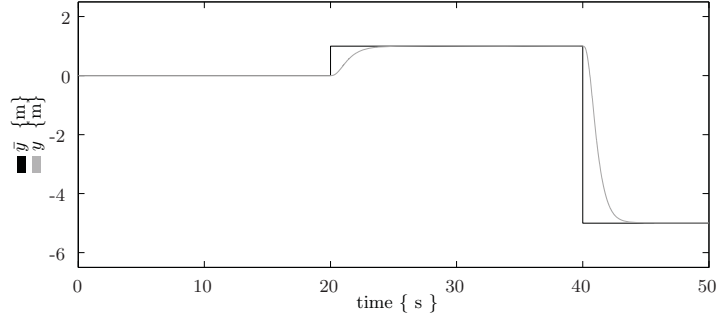

(b) $y$-position

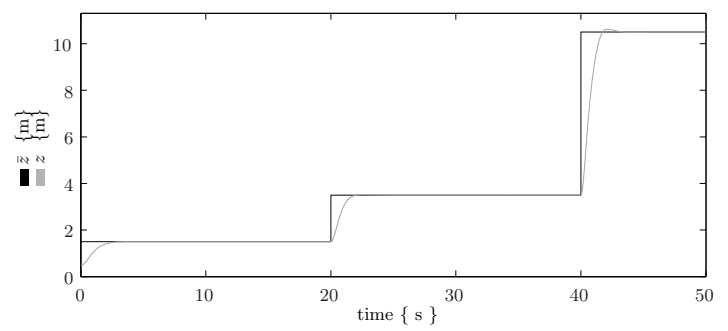

(c) $z$-position

Figure A.4: Position of the DFMAV in set-point stabilization task.

\section{A.4.2 Simulation Results}

In this sextion validating simulation results of both hovering and tracking tasks using the DFMAV are given. To test the robustness of the proposed controller, different nominal and actual inertial parameters and gravity are used in all simulations.

\section{A.4.2.1 Simulation I - Hovering}

In this simulation, the UAV starts at an initial position $(0,0,0.5)$ and hovers around the following three points, while the yaw attitude is kept at zero, i.e.,

$$
\begin{cases}p 1=(0,0,1.5) & \text { for } t<20 \\ p 2=(3,1,3.5) & \text { for } 20 \leq t \leq 40 \\ p 3=(-7,-5,10.5) & \text { for } t>40\end{cases}
$$

The results shown in Fig. A.4 illustrate that the controller is able to stabilize the DFMAV at the three desired positions as expected.

\section{A.4.2.2 Simulation II - Trajectory tracking}

In this simulation, the task performed includes both trajectory tracking and stabilization at stationary points, in the presence of initial configuration error and bounded disturbances. The main goal of this simulation is to demonstrate how effective the controller is in different flight regimes and its robustness even in the presence of disturbance and uncertainties. 


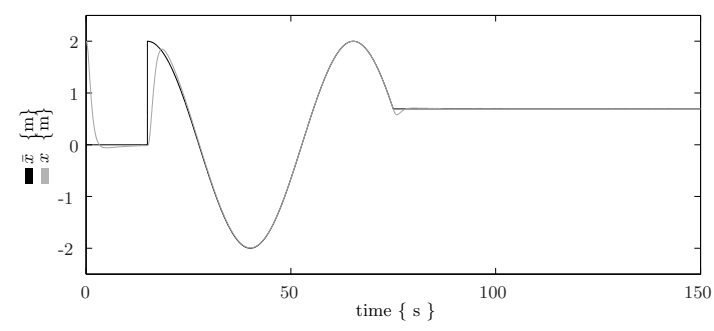

(a) $x$-position

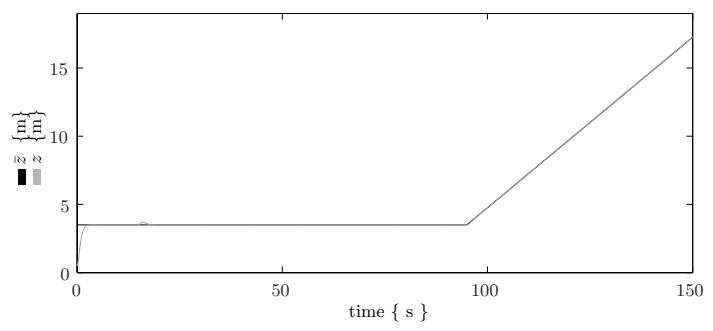

(c) $z$-position

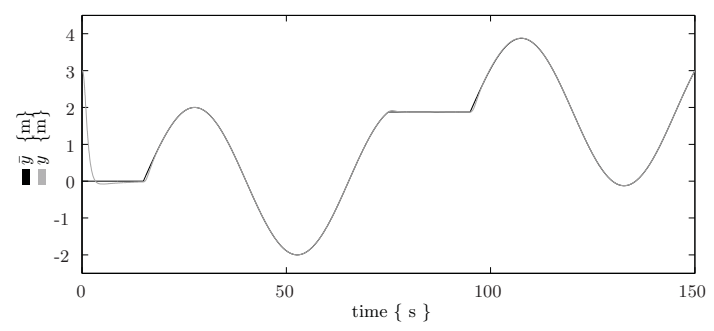

(b) $y$-position

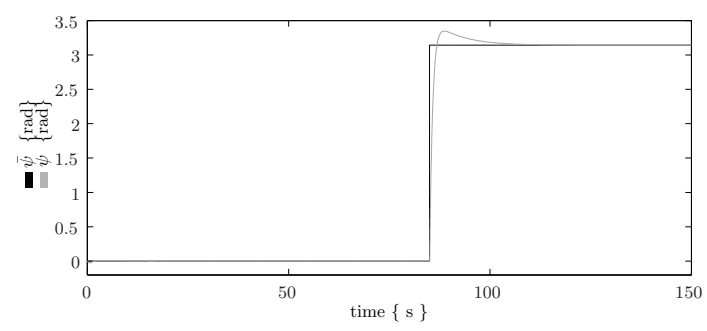

(d) yaw

Figure A.5: Position and yaw of the DFMAV in trajectory tracking task.

The initial position of the DFMAV is $(2,3,0.5)$, and the desired trajectory profiles are

$$
\begin{aligned}
& x(t)= \begin{cases}0 & 0<t \leq 15 \\
2 \cos \left(\frac{t-15}{8}\right) & 15<t \leq 75 \\
2 \cos \left(\frac{60}{8}\right) & t>75\end{cases} \\
& y(t)= \begin{cases}0 & 0<t \leq 15 \\
2 \sin \left(\frac{t-15}{8}\right) & 15<t \leq 75 \\
2 \sin \left(\frac{60}{8}\right) & 75<t \leq 95 \\
2 \cos \left(\frac{t-95}{8}\right)+2 \sin \left(\frac{60}{8}\right) & t>95\end{cases} \\
& z(t)= \begin{cases}3.5 & 0<t \leq 95 \\
0.25(t-95) & t>95\end{cases} \\
& \psi(t)= \begin{cases}0 & t \leq 95 \\
\pi & t>95\end{cases}
\end{aligned}
$$

The results shown in Fig. A.5 illustrate that, despite the presence of parametric uncertainty, measurement error and disturbance, the controller manages to robustly track the reference trajectories.

\section{A.5 Conclusions}

In this appendix, a generic dynamic model for a class of underactuated UAV and an intuitive controller design for hovering and trajectory tracking tasks have been presented. The mathematical tool of port-Hamiltonian system, together with the 
graphical modeling language of bond graph, was used to exploit the structural information of the physical system and provide more insight. Applicability of both the generic dynamic model and controller to specific platforms was shown in a case study via simulation. 


\section{APPENDIX B}

\section{PARAMETER TUNing}

The reliability performance of the virtual vehicle is measured based on how close its states are to the states of the real slave, provided that there is energy in the primary energy tank. To achieve a higher reliability performance, the parameters of the virtual slave system should be tuned carefully.

For ease of understanding the procedure, consider a one dimensional representation of the virtual vehicle and the real slave, see Fig. B.1. $d_{r}$ represents the viscous damping coefficient of the real slave; $F_{c}$ and $F_{d}$ represent the controller action of the virtual slave and the disturbance force applied on the real slave. In this analysis it is assumed that the low-level controller of the real slave compensates for gravity and other aerodynamic effects. Besides, the analysis is for operating conditions where there is adequate energy in the primary energy tank.

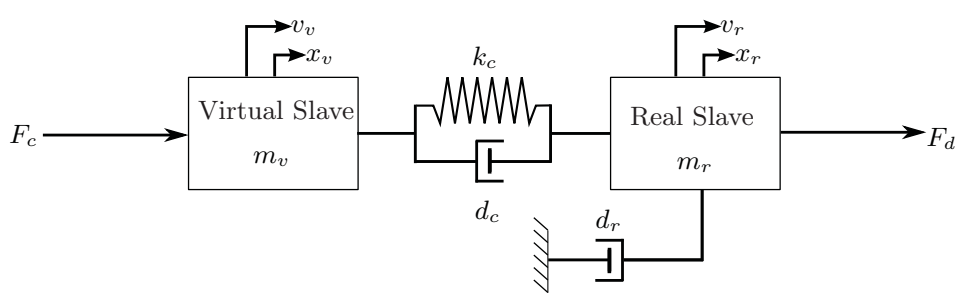

Figure B.1: Simplified 1D representation of the virtual and real slaves.

The dynamic map from $F_{c}$ and $F_{d}$ to the positions $x_{v}$ and $x_{r}$ and the velocities $v_{v}$ and $v_{r}$ is given by

$$
\left(\begin{array}{l}
x_{v} \\
x_{r} \\
v_{v} \\
v_{r}
\end{array}\right)=\left(\begin{array}{ll}
H_{11} & H_{12} \\
H_{21} & H_{22} \\
H_{31} & H_{32} \\
H_{41} & H_{42}
\end{array}\right)\left(\begin{array}{l}
F_{c} \\
F_{d}
\end{array}\right)
$$


where $H_{i j}$, for $i=1,2,3,4$ and $j=1,2$ are the transfer equations and are given by

$$
\begin{gathered}
H_{11}=\frac{1}{s} H_{31}=\frac{m_{r} s^{2}+\left(d_{c}+d_{r}\right) s+k_{c}}{d(s)} \\
H_{12}=H_{21}=\frac{1}{s} H_{32}=\frac{1}{s} H_{41}=\frac{d_{c} s+k_{c}}{d(s)} \\
H_{22}=\frac{1}{s} H_{42}=\frac{m_{v} s^{2}+d_{c} s+k_{c}}{d(s)}
\end{gathered}
$$

where $d(s)=m_{v} m_{r} s^{3}+\left\{\left(m_{v}+m_{v}\right) d_{c}+m_{v} d_{r}\right\} s^{2}+\left\{\left(m_{v}+m_{r}\right) k_{c}+d_{c} d_{r}\right\} s+k_{c} d_{r}$.

From (B.1), $x_{v} \approx x_{r}$ and $v_{v} \approx v_{r}$ can be deduced if $H_{11} \approx H_{21}, H_{12} \approx H_{22}$ and $H_{31} \approx H_{41}, H_{32} \approx H_{42}$. To guarantee the state correspondence between the two slaves therefore, the magnitudes of the virtual slave parameters should be tuned as follows:

$$
\begin{gathered}
m_{v}<<m_{r}, d_{c}>>d_{r}, d_{c}>>m_{r}, \\
k_{c}>>d_{r} \text { and } k_{c}>>m_{r}
\end{gathered}
$$

Note that while selecting $d_{c}$ and $k_{c}$, limitations due to actuator's saturation must also be taken into account. 


\section{APPENDIX $C$}

\section{Proof of Proposition 4.3}

Following [76], consider a candidate Lyapunonve function $V(\Lambda)=\Lambda^{T} \Sigma \Lambda$, where

$$
\begin{aligned}
& \Lambda^{T}=\left[\left|e_{1}^{i}\right|^{1 / 2} \operatorname{sign}\left(e_{1}^{i}\right) \quad e_{1}^{i} e_{2}^{i}\right] \\
& \Sigma=\frac{1}{2}\left[\begin{array}{ccc}
\left(4 k_{2}+k_{1}^{2}\right) & k_{1} L_{1} & -k_{1} \\
k_{1} L_{1} & \left(2 L_{2}+L_{2}^{2}\right) & -L_{1} \\
-k_{1} & -L_{1} & 2
\end{array}\right]
\end{aligned}
$$

$V(\Sigma)$ has the following characteristics

i) The symmetric matrix $\Sigma$ is positive definite for $k_{1}, k_{2}>0, L_{1}, L_{2}>0$. Thus, $V(\Sigma)$ is a continuous, radially unbounded positive definite function and satisfies

$$
\lambda_{\min }\{\Sigma\}\|\Lambda\|^{2} \leq V(\Sigma) \leq \lambda_{\max }\{\Sigma\}\|\Lambda\|^{2}
$$

where $\|\Lambda\|=\left|e_{1}^{i}\right|+\left(e_{1}^{i}\right)^{2}+\left(e_{2}^{i}\right)^{2}$.

ii) $V(\Sigma)$ is continuously differentiable except at $e_{1}=0$.

Note also the following relations for $\left\{x \in \mathbb{R}^{3} \mid x \neq 0\right\}$,

- $\operatorname{sign}(x)=\frac{x}{|x|}$

- $x \operatorname{sign}(x)=\left(|x|^{1 / 2} \operatorname{sign}(x)\right)^{2}$

- $\frac{d}{d t}\left(|x|^{1 / 2} \operatorname{sign}(x)\right)=\frac{1}{2|x|^{1 / 2}} \dot{x}$

Now, taking the time derivative of $V(\Sigma)$ results in

$$
\begin{aligned}
\dot{V} & =\dot{\Lambda}^{T} \Sigma \Lambda+\Lambda^{T} \Sigma \dot{\Lambda} \\
& =-\frac{1}{\left|e_{1}^{i}\right|^{1 / 2}} \Lambda^{T} \Delta_{1} \Lambda-\Lambda^{T} \Delta_{2} \Lambda-\frac{2 d^{i}}{m^{i}}\left(e_{2}^{i}\right)^{2}+\Gamma^{T} \Lambda
\end{aligned}
$$


where

$$
\begin{aligned}
& \Delta_{1}=\frac{k_{1}}{2}\left[\begin{array}{ccc}
\left(2 k_{2}+k_{1}^{2}\right) & 0 & -k_{1} \\
0 & \left(2 L_{2}+5 L_{1}^{2}\right) & -\left(3 L_{1}+\frac{d^{i}}{m^{i}}\right) \\
-k_{1} & -\left(3 L_{1}+\frac{d^{i}}{m^{i}}\right) & 1
\end{array}\right] \\
& \Delta_{2}=L_{1}\left[\begin{array}{ccc}
\left(k_{2}+2 k_{1}^{2}\right) & 0 & 0 \\
0 & \left(L_{2}+L_{1}^{2}\right) & -\left(L_{1}+\frac{d^{i}}{2 m^{i}}\right) \\
0 & -\left(L_{1}+\frac{d^{i}}{2 m^{i}}\right) & 1
\end{array}\right] \\
& \Gamma^{T}=-v^{i}\left[\begin{array}{ccc}
\frac{k_{1}}{m^{i}} & \frac{L_{1}}{m^{i}} & -\frac{2}{m^{i}}
\end{array}\right]
\end{aligned}
$$

Since $v^{i}$ is bounded, i.e., $\left|v^{i}\right|<\rho^{i}$, it is easy to verify the following inequality.

$$
\Gamma^{T} \Lambda \leq \frac{1}{\left|e_{1}^{i}\right|^{1 / 2}} \Lambda^{T} \Delta_{3} \Lambda+\Lambda^{T} \Delta_{4} \Lambda
$$

where,

$$
\begin{aligned}
& \Delta_{3}=\frac{k_{1}}{2}\left[\begin{array}{ccc}
\frac{2 \rho^{i}}{m^{i}} & 0 & \frac{2 \rho^{i}}{m^{i} k_{1}} \\
0 & 0 & 0 \\
\frac{2 \rho^{i}}{m^{i} k_{1}} & 0 & 0
\end{array}\right] \\
& \Delta_{4}=L_{1}\left[\begin{array}{ccc}
\frac{\rho^{i}}{m^{i}} & 0 & 0 \\
0 & 0 & 0 \\
0 & 0 & 0
\end{array}\right]
\end{aligned}
$$

From (C.2) and (C.3), one can deduce

$$
\dot{V} \leq-\frac{1}{\left|e_{1}^{i}\right|^{1 / 2}} \Lambda^{T} \Delta_{5} \Lambda-\Lambda^{T} \Delta_{6} \Lambda
$$

where $\Delta_{5}=\Delta_{1}-\Delta_{3}$ and $\Delta_{6}=\Delta_{2}-\Delta_{4}$.

To shown that $\dot{V}$ is negative definite, it is sufficient to show that $\Delta_{5}$ and $\Delta_{6}$ are positive definite matrices. These matrices are positive definite for the following observer parameters

$$
\begin{aligned}
& k_{1}>0 \\
& k_{2}>\frac{3 \rho^{i}}{m^{i}}+2\left(\frac{\rho^{i}}{k_{1} m^{i}}\right)^{2} \\
& L_{1}>0 \\
& L_{2}>\frac{1}{2}\left(3 L_{1}+\frac{d^{i}}{m^{i}}\right)^{2}
\end{aligned}
$$

Provided that (C.5) is fulfilled, the following relation holds.

$$
\begin{aligned}
\dot{V} & \leq-\frac{1}{\left|e_{1}^{i}\right|^{1 / 2}} \Lambda^{T} \Delta_{5} \Lambda \\
& \leq-\frac{1}{\left|e_{1}^{i}\right|^{1 / 2}} \lambda_{\min }\left\{\Delta_{5}\right\}\|\Lambda\|^{2}
\end{aligned}
$$

From the relation given in (C.1) and the fact that $\Delta_{5}$ is positive definite for observer values fulfilling (C.5), it readily follows that

$$
\dot{V}(\Lambda) \leq-\alpha V^{1 / 2}(\Lambda)
$$


where

$$
\alpha=\frac{\lambda_{\min }^{1 / 2}\{\Sigma\} \lambda_{\min }\left\{\Delta_{5}\right\}}{\lambda_{\max }^{1 / 2}\{\Sigma\}}
$$

To estimate the convergence time, consider the solution of the differential equation

$$
\dot{x}=-\alpha x^{1 / 2}, \text { for } \alpha>0 \text { and } x(0)>0
$$

which is

$$
x(t)=\left(x^{1 / 2}(0)-\frac{\alpha}{2} t\right)^{t}
$$

Following the comparison principle in [44], $V(t)<x(t)$ for $V(\Lambda(0))<x(0)$. Hence, $V(\Lambda(t))$, thereby, $\Lambda(t)$ converges to the origin with in finite time $T=$ $\frac{2 V^{1 / 2}(\Lambda(0))}{\alpha}$. 



\section{BIBLIOGRAPHY}

[1] Agrachev, A. A., Morse, A. S., Sontag, E. D., Sussmann, H. J., And Utkin, V. I. Nonlinear and Optimal Control Theory. Springer-Verlag, Berlin Heidelberg, 2008.

[2] Ajoudani, A., Tsagarakis, N. G., And Bicchi, A. Tele-impedance: Teleoperation with impedance regulation using a body-machine interface. The International Journal of Robotics Reserach 31, 13 (2012), 1643-1656.

[3] Alexis, K., Huerzeler, C., and Seigwart, R. Hybrid modeling and control of a coazial unmanned rotorcraft interacting with its environment through contact. In Proceedings of the IEEE International Conference on Robotics and Automation (2013).

[4] Aziminejad, A., Tavakoli, M., Patel, R., and Moallem, M. Transparent Time-Delayed Bilateral Teleoperation Using Wave Variables. IEEE Transactions on Control Systems Technology 16, 3 (May 2008), 548-555.

[5] Beelen, M. J., Naus, G. J., van de Molengraft, M. R. J., And Steinbuch, M. Force feedback control design for nonideal teleoperators. Control Engineering Practice 21, 12 (2013), 1694-1705.

[6] Bejarano, F., Poznyak, A., And Fridman, L. hierarchical second-order sliding mode observer for linear time invariant systems with unknown inputs. International Journal of System Science 38, 10 (2007), 793-802.

[7] Bellens, S., De Schutter, J., And Bruyninckx, H. A hybrid pose / wrench control framework for quadrotor helicopters. In Proceedings of the IEEE International Conference on Robotics and Automation (2012).

[8] Blank, A., Okamura, A., And Whitcomb, L. Task-dependent impedance improves user performance with a virtual prosthetic arm. In Proceedings of the IEEE International Conference on Robotics and Automation (2011).

[9] Burri, M., Nikolic, J., Hurzeler, C., Caprari, G., and Siegwart, R. Aerial service robots for visual inspection of thermal power plant boiler 
systems. In Proceedings of the International Conference on Applied Robotics for the Power Industry (2012).

[10] Carloni, R., Lippiello, V., D’Auria, M., Fumagalli, M., Mersha, A., Stramigioli, S., And Siciliano, B. Robot vision: Obstacle-avoidance techniques for unmanned aerial vehicles. IEEE Robotics Automation Magazine 20, 4 (Dec 2013), 22-31.

[11] Chen, S.-F., And KaO, I. Conservative congruence transformation for joint and cartesian matrices of robot hands and fingures. The International Journal of Robotics Reserach 19, 9 (2000), 835-847.

[12] Chopra, N., Spong, M. W., Hirche, S., And Buss, M. Bilateral teleoperation over the internet: the time varying delay problem. In Proceedings of the American Control Conference (2003).

[13] Colgate, E., And Hogan, N. An analysis of contact instability in terms of passive physical equivalents. In Proceedings of the IEEE International Conference on Robotics and Automation (1989).

[14] Diolaiti, N., And Melchiorri, C. Haptic tele-operation of a mobile robot. In Proceedings of the IFAC Symposium on Robot Control (2003).

[15] Duindam, V., Macchelli, A., Stramigioli, S., And H. Bruyninckx, E. Modeling and control of complex physical systems. Springer Verlag, 2009.

[16] Dzul, A., Hamel, T., And Lozano, R. Modeling and nonlinear control for a coaxial helicopter. In Proceedings of the IEEE International Conference on Systems, Man and Cybernetics (2002).

[17] Erickson, D., Webber, M., And Sharf, I. Contact Impedance Estimation for Robotic Systems. The International Journal of Robotics Research 22, 1 (2003), 41-57.

[18] Farkhatdinov, I., and Ryu, J.-H. Switching of Control Signals in Teleoperation Systems: Formalization and Application. In IEEE/ASME Int. Conference on Advanced Intelligent Mechatronics (2008).

[19] Ferraguti, F., Secchi, C., And Fantuzzi, C. A tank-based approach to impedance control with variable stiffness. In Proceedings of the IEEE International Conference on Robotics and Automation (2013).

[20] Fong, T., And Thorpe, C. Vehicle Teleoperation Interfaces. Kluwer Academic Publishers, 2001.

[21] Forte, F., Naldi, R., Macchelli, A., And Marconi, L. Impedance control of an aerial Manipulator. In Proceedings of the American Control Conference (2012). 
[22] Franchi, A., Giordano, P. R., Secchi, C., Son, H., and Bulthoff, H. A passivity-based decentralized approach for the bilateral teleoperation of a group of uavs with switching topology. In Proceedings of the IEEE International Conference on Robotics and Automation (2011).

[23] Franken, M. C. J. Control of Haptic Interaction: An Energy-Based Approach. PhD thesis, University of Twente, 2011.

[24] Franken, M. C. J., Misra, S., and Stramigioli, S. Friction compensation in energy-based bilateral telemanipulation. In Proceedings of IEEE/RSJ International Conference on Intelligent Robots and Systems (2010).

[25] Fumagalli, M., And Carloni, R. A modified impedance control for physical interaction of UAVs. In Proceedings of the IEEE/RSJ International Conference on Intelligent Robotic Systems (2013).

[26] Fumagalli, M., Naldi, R., Macchelli, A., Carloni, R., Stramigioli, S., AND MARCONI, L. Modeling and control of a flying robot for contact inspection. In Proceedings of the IEEE International Conference on Intelligent Robots and Systems (2012).

[27] Goertz, R. Mechanical master-slave manipulator. Nucleonics (1954), 4546.

[28] Handlykken, M., And Turner, T. Control system analysis and synthesis for a six degree-of-freedom universal force-reflecting hand controller. In Preceedings of the IEEE International Conference on Decision and Control including the Symposium on Adaptive Processes (1980).

[29] Hannaford, B. A design framwork for teleoperators with kinestetic feedback. IEEE Transaction in Robotics and Automation 5, 4 (Dec. 1989), 426434 .

[30] Hannaford, B., And Ryu, J. Time-domain passivity control of haptic interfaces. IEEE Transactions on Robotics and Automation 18, 1 (2002), $1-10$.

[31] Hirabayashi, T., Yamamoto, Yano, H., and Iwata, H. Experiment on teleoperation of underwater backhoe with haptic information. In Proceedings of the International Symposium on Automation and Robotics in Construction (2006).

[32] Hogan, N. Impedance control: an approach to manipulation: parts i-iii. ASME Journal of Dynamic Systems, Measurement and Control 10\%, 1 (aug 1985), 1-24.

[33] Hollis, R., ANd Salcudean, S. Lorentz levitation technology: a new approach to fine motion robotics, teleoperation, haptic interfaces and vibration isolation. In Proceedings of the International Symposium on Robotics Research (1993). 
[34] Hou, X., And Mahony, R. Dynamic kinesthetic boundary for haptic teleoperation of aerial robotic vehicles. In Proceedings of IEEE/RSJ International Conference on Intelligent Robots and Systems (2013).

[35] Hou, X., And Mahony, R. An intuitive multimodal haptic interface for teleoperation of aerial robots. In Proceedings of the IEEE International Conference on Robotics and Automation (2014).

[36] Hou, X., Mahony, R., And Schill, F. Representation of Vehicle Dynamics in Haptic Teleoperation of Aerial Robots. In Proceedings of the Australasian Conference on Robotics and Automation (2013).

[37] Hua, M. D., Hamel, T., Morin, P., and Samson, C. A control approach for thrust-propelled underactuated vehicles and its application to VTOL drones. IEEE Transaction on Automatic Control 54, 8 (2009), 18371853.

[38] Hunt, K., And Crossley, F. Coefficient of restitution interpreted as damping in vibroimpact. The ASME Journal of Applied Mechanics 42 (June 1975), 440-445.

[39] HürZELER, C. Modeling and design of unmanned rotorcraft systems for contact based inspection. PhD thesis, The Swiss Federal Institute of Technology (ETH, Zürich), 2013.

[40] Isidori, A. Nonlinear Control Systems, 3rd ed. Springer-Verlag New York, Inc., 1995.

[41] Kawashima, K., Tadano, K., Sankaranarayanan, G., and HanNAFORD, B. Model-based passivity control for bilateral teleoperation of a surgical robot with time delay. In Proceedings of IEEE/RSJ International Conference on Intelligent Robots and Systems (2008).

[42] Keemink, A. Design, realization and analysis of a manipulation system for uavs. Master's thesis, University of Twente, 2012.

[43] Keemink, A., Fumagalli, M., Stramigioli, S., and Carloni, R. Mechanical design of manipulation system for unmanned aerial vehicle. In Proceedings of the IEEE International Conference on Robotics and Automation (2012).

[44] Khalil, H. Nonlinear Systems, 3rd ed. Prentice Hall, 2002.

[45] Kim, J., Chang, P. H., And Park, H.-S. Two-channel transparency optimized control architecture in bilateral teleoperation with time delay. IEEE Transaction in Control Systems Technology 21, 1 (Jan. 2013), 40-51.

[46] Kristoffersson, A., Coradeschi, S., And Loutfi, A. A Review of Mobile Robotic Telepresence. Advances in Human-Computer Interaction (2013), $1-17$. 
[47] Lam, T., Boschloo, H., Mulder, M., and van Paassen, M. Artificial force field for haptic feedback in uav teleoperation. IEEE Transactions on Systems, Man and Cybernetics, Part A: Systems and Humans 39, 6 (2009), 1316-1330.

[48] Lam, T., Mulder, M., and van Paassen, M. Haptic feedback for UAV tele-operation - force offset and spring load modification. In Proceedings of the International Conference on Systems, Man and Cybernetics (2006).

[49] Lam, T., Mulder, M., and van PaAssen, M. Haptic Feedback in uninhabited aerial vehicle teleoperation with time delay. Journal of Guidance, Control, and Dynamics 31, 6 (2008), 1728-1739.

[50] Lawrence, D. A. Designing teleoperator architectures for transparency. In Proceedings of the IEEE International Conference on Robotics and Automation (1992).

[51] LaWrence, D. A. Stability and transparency in bilateral teleoperation. IEEE Transactions on Robotics and Automation 9, 5 (March 1993), 624-637.

[52] Lazaro, J., Abuan, D., Linsangan, N., And Panganiban, A. Surface electromyography signal for control of myoelectric prosthesis of the upperlimb using independent component analysis. Journal of Automation and Control Engineering 2, 1 (2014).

[53] Lee, D., Franchi, A., Robuffo Giordano, P., Son, H., And Bülthoff, H. Haptic teleoperation of multiple unmanned aerial vehicles over the internet. In Proceedings of the IEEE International Conference on Robotics and Automation (2011).

[54] LeE, D., AND Xu, D. Feedback r-passivity of Lagrangian systems for mobile robot teleoperation. In Proceedings of the IEEE International Conference on Robotics and Automation (2011).

[55] LeE, K., ANd Buss, M. Force tracking impedance control with variable target stiffness. In Proceedings of the IFAC World Congress (2008).

[56] Levine, J. Analysis and Control of Nonlinear Systems, vol. 115. SpringerVerlag Berlin Heidelberg, 2009.

[57] Lim, J.-N., Ko, J.-P., AND LEE, J.-M. Internet-based teleoperation of a mobile robot with force reflection. In Proceedings of the IEEE International Conference on Robotics and Automation (2006).

[58] Mahony, R., Schill, F., Corke, P., and Oh, Y. A new framework for force feedback teleoperation of robotic vehicles based on optical flow. In Proceedings of the IEEE International Conference on Robotics and Automation (2009). 
[59] Marconi, L., Basile, L., Caprari, G., Carloni, R., Chiacchio, P., Huerzeler, C., Lippiello, V., Naldi, R., Janosch, N., Siciliano, B., Stramigioli, S., AND Zwicker, E. Aerial service robotics: the airobots perspective. In Proceedings of the IEEE International Conference on Applied Robotics for the Power Industry (2012).

[60] Marconi, L., And NAldi, R. Robust full degree-of-freedom tracking control of a helicopter. Automatica 43, 11 (Nov. 2007), 1909-1920.

[61] Marconi, L., And Naldi, R. Control of aerial robots: Hybrid force and position feedback for a ducted fan. Control Systems, IEEE 32, 4 (Aug 2012), $43-65$.

[62] Maza, I., Kondak, K., Bernand, M., and Ollero, A. Multi-UAV Cooperation and Control for Load Transportation and Deployment. Journal of Intelligent and Robotic Systems 57, 1 (2010), 417-449.

[63] Mellinger, D., and Kumar, V. Minimum snap trajectory generation and control for quadrotors. In Proceedings of the IEEE International Conference on Robotics and Automation (2011).

[64] Mellinger, D., Shomin, M., And Kumar, V. Control of Quadrotors for Robust Perching and Landing. In International Powered Lift Conference (2006).

[65] Mersha, A. Y. Modeling and robust control of unmanned aerial vehicles. Master's thesis, University of Twente, 2010.

[66] Mersha, A. Y., Carloni, R., and Stramigioli, S. Modeling and control of underactuated aerial vehicles. In Proceedings of the IEEE International Conference on Robotics and Automation (2011).

[67] Mersha, A. Y., Hou, X., Mahony, R., Stramigioli, S., Corke, P., AND CARLONI, R. The longest intercontinental teleoperation of flying robot: a step towards real-time application. In Proceedings of the IEEE/RSJ International Conference on Intelligent Robots and Systems (2013).

[68] Mersha, A. Y., Rüesch, A., Stramigioli, S., And Carloni, R. A Contribution to Haptic Teleoperation of Aerial Vehicles. In Proceedings of the IEEE/RSJ International Conference on Intelligent Robots and Systems (2012c).

[69] Mersha, A. Y., Stramigioli, S., and Carloni, R. Bilateral teleoperation of underactuated aerial vehicles: the virtual slave concept. In Proceedings of the IEEE International Conference on Robotics and Automation (2012a).

[70] Mersha, A. Y., Stramigioli, S., and Carloni, R. Switching-based Mapping and Control for Haptic Teleoperation of Aerial Robots. In Proceedings of the IEEE/RSJ International Conference on Intelligent Robots and Systems (2012b). 
[71] Mersha, A. Y., Stramigioli, S., and Carloni, R. On bilateral teleoperation of aerial robots. IEEE Transactions on Robotics 30, 1 (Feb 2014a), $258-274$.

[72] Mersha, A. Y., Stramigioli, S., and Carloni, R. Exploiting the dynamics of a robotic manipulator for control of UAVs. In Proceedings of the IEEE International Conference on Robotics and Automation (2014b).

[73] Mersha, A. Y., Stramigioli, S., And Carloni, R. A variable impedance control for aerial interaction. In Proceedings of the IEEE/RSJ International Conference on Intelligent Robots and Systems (2014c).

[74] Minelu, M., And Podobnik, J. Haptics for Virtual Reality and Teleoperation, 1st ed. Springer-Dordrecht Heidelberg, 2012.

[75] Mobasser, F., and Hashtrudi-ZaAd, K. A method for online estimation of human arm dynamics. In Proceedings of the $28^{\text {th }}$ Annual International Conference of the IEEE Engineering in Medicine and Biology Society (2006).

[76] Moreno, J., And Osorio, M. A lyapunov approach to second-order sliding mode controllers and observers. In Proceedings of the IEEE Conference on Decision and Control (2008).

[77] Munir, S., AND Book, W. J. Internet based teleoperation using wave variables. In Proceedings of the IEEE/ASME International Conference on Advanced Mechatronics (2001).

[78] Murray, R. M., Sastry, S. S., And Zexiang, L. A Mathematical Introduction to Robotic Manipulation, 1st ed. CRC Press, Inc., 2.

[79] Naerum, E., And Hannafrod, B. Global transparency analysis of the lawrence teleoperator acrhitecture. In Proceedings of the IEEE International Conference on Robotics and Automation (2009).

[80] Naldi, R., Gentili, L., Marconi, L., And Sala, A. Design and experimental validation of a nonlinear control law for ducted fan miniature aerial vehicle. Control Engineering Practice 18, 7 (2010), 677-836.

[81] Naldi, R., Torre, A., And Marconi, L. Robust blind navigation for miniature ducted-fan aerial robot. In Proceedings of the American Control Conference (2013).

[82] Niemeyer, G., And Slotine, J. J. E. Telemanipulation with Time Delayes. The International Journal of Robotics Reserach 23, 9 (2004), 873-890.

[83] NiJmeijer, H., AND VAN DER Schaft, A. Nonlinear dynamical control systems. Springer-Verlag New York, Inc., 1990. 
[84] Omari, S., Hua, M.-D., Ducard, G., and Hamel, T. Bilateral haptic teleoperation of vtol uavs. In Proceedings of the IEEE International Conference on Robotics and Automation (2013).

[85] Orsag, M., Korpela, C., And Oh, P. Modeling and control of mm-uav: mobile manipulating unmanned aerial vehicle. Journal of Intelligent and Robotic Systems (2013), 227-240.

[86] Ortega, R., van der Schaft, A. J., Mareels, I., And Maschke, B. Putting energy back in control. IEEE Control Systems Magazine 21, 2 (April 2001), 18-33.

[87] Palunko, I., Cruz, P., And Fierro, R. Agile Load Transportation: Safe and Efficient Load Manipulation with Aerial Robots. IEEE Robotic and Automation Magazine 19, 3 (2012), 69-79.

[88] Panteley, E., And Loria, A. Growth rate conditions for stability of cascaded time-varying systems. Automatica 37, 3 (2001), 453-460.

[89] Pflimlin, J., Hamel, T., Soueres, P., And Mahony, R. A hierarchical control strategy for the autonomous navigation of a ducted fan flying robot. In Proceedings of the IEEE International Conference on Robotics and Automation (2006).

[90] Pounds, P., Bersak, D. R., And Dollar, A. M. Stability of small-scale uav helicopters and quadrotors with added payload mass under pid control. Autonomous Robots 33, 1-2 (Aug. 2012), 129-142.

[91] Pounds, P., Mahony, R., And Corke, P. Modelling and control of a large quadrotor robot. Control Engineering Practice 18, 7 (Feb. 2010), 691-699.

[92] Raptis, I. A., And Valavanis, K. P. Linear and Nonlinear Control of Small-Scale Unmanned Helicopters. Springer, 2011.

[93] Razi, K., Yazdanpanah, M.-J., Ghidary, S., Najafabadi, H., Zareinejad, M., Seifabadi, R., And Rezaei, S. Position coordination of a linear teleoperation system with constant time delay. In Proceedings of the IEEE/RSJ International Conference on Intelligent Robots and Systems (2007)

[94] Rifä̈, H., Duc Hua, M., Hamel, T., and Morin, P. Haptic-based bilateral teleoperation of underactuated unmanned aerial vehicles. In Proceedings of the IFAC World Congress (2011).

[95] Roy, J., And Whitcomb, L. Adaptive force control of position/velocity controlled robots: theory and experiment. IEEE Transactions on Robotics and Automation 18, 2 (2002), 121-137. 
[96] R.Suzuki, Matsumoto, T., Konno, A., Hoshino, Y., Go, K., Oosedo, A., And UChIYAma, M. Teleoperation of a tail-sitter VTOL UAV. In Proceedings of the IEEE/RSJ International Conference on Intelligent Robots and Systems (2010).

[97] Rüesch, A., Mersha, A. Y., Stramigioli, S., and Carloni, R. Kinetic scrolling-based Position Mapping for Haptic Teleoperation of Unmanned Aerial Vehicles. In Proceedings of the IEEE International Conference on Robotics and Automation (2012).

[98] Ryll, M., Bülthoff, H., And Giordano, P. First flight tests for a quadrotor uav with tilting propellers. In Proceedings of IEEE International Conference on Robotics and Automation (2013).

[99] Salcudean, S. E., Ku, S., And Bell, G. Performance measurement in scaled teleoperation for microsurgery. First Joint Conference of Computer Vision, Virtual Reality, and Robotics in Medicine and Medical Robotics and Computer-Assisted Surgery (March 1997 1997), 789-798.

[100] Salcudean, S. E., Zhu, M., Zhu, W.-H., And Hashtrudi-ZaAd, K. Transparent bilateral teleoperation under position and rate control. The International Journal of Robotics Research 19, 12 (Dec. 2000), 1185-1202.

[101] Schill, F., Hou, X., And Mahony, R. Admittance mode framework for haptic teleoperation of hovering vehicles with unlimited workspace. In Proceedings of the Australasian Conference on Robotics and Automation (2010).

[102] Scholten, J. L., Fumagalli, M., Stramigioli, S., and Carloni, R. Interaction Control of an UAV Endowed with a Manipulator. In Proceedings of the IEEE International Conference on Robotics and Automation (2013).

[103] Secchi, C., Stramigioli, S., And Fantuzzi, C. Control of Interactive Robotic interfaces: A Port-Hamiltonian Approach. Springer Verlag, 2007.

[104] Selen, L., van DieËn JH, AND Beek, P. Impedance modulation and feedback corrections in tracking targets of variable size and frequency. Journal of Neurophysiology 96 (2006), 2750-2759.

[105] Seraji, H., And Richard, C. Force tracking in impedance control. In Proceedings of the IEEE International Conference on Robotics and Automation (1993).

[106] Sheridan, T. B. Space teleoperation through time delay: review and prognosis. IEEE Transaction in Robotics and Automation 9, 5 (Oct. 1993), 592606.

[107] Siciliano, B., And Khatib, Q., Eds. Springer Handbook of Robotics. Springer, 2008. 
[108] Siegwart, R., And Nourbakhsh, I. R. Introduction of autonomouse mobile robots, 2nd ed. The MIT press, 2004.

[109] Stramigioli, S. Modeling and IPC Control of Interactive Mechanical Systems: A Coordinate-free Approach. Springer Verlag, 2001.

[110] Stramigioli, S., Fasse, E. D., And Willems, J. C. A rigorous framework for interactive robot control. International Journal of Control 75, 11 (2002), $1486-1503$.

[111] Stramigioli, S., Mahony, R., And Corke, P. A novel approach to haptic tele-operation of aerial robot vehicles. In Proceedings of the IEEE International Conference on Robotics and Automation (2010).

[112] Tavakoli, M., Patel, R., Moallem, M., and Aziminejad, A. Haptics for Teleoperated Surgical Robotic Systems. 2008.

[113] Torre, A., Naldi, R., Ricco, A., Mengoli, D., and Marconi, L. An over-actuated modular platform for aerial inspection and manipulation. In Proceedings of the IEEE/RSJ International Conference on Intelligent Robots and Systems (2013).

[114] Tzafestas, C., Velanas, S., and Fakiridis, G. Adaptive impedance control in haptic teleoperation to improve transparency under time-delay. In Proceedings of the IEEE International Conference in Robotics and Automation (2008).

[115] Utkin, V. Sliding modes in control optimizations. Springer Verlag, 1992.

[116] Veluvolu, K. C., And Chai, Y. High-gain observers with sliding mode for state and unknown input estimations. IEEE Transactions on Industrial Electronics 56, 9 (sept 2009), 3386-3393.

[117] Walker, D., Salisbury, J., And Niemeyer, G. Demonstrating the benefits of variable impedance to telerobotic task execution. In Proceedings of the IEEE International Conference on Robotics and Automation (2011).

[118] Yokоконлi, Y., And Yoshikawa, T. Bilateral Control of Master-Slave Manipulators for Ideal Kinesthetic Coupling-Formulation and Experiment. In Proceedings of the IEEE International Conference on Robotics and Automation (1992). 


\section{About the Author}

Abeje Yenehun Mersha was born on August 14 $4^{\text {th }}$ 1984, in Addis Ababa, Ethiopia. He attended his elementary and secondary education at Lideta Catholic Cathedral School, Addis Ababa, Ethiopia. After scoring straight A's in his Matriculation, he joined Mekelle University in 2002 to pursue his undergraduate studies in Electrical Engineering. He received his BSc. degree with Very Great Distinction from Mekelle Univerity, Mekelle, Ethiopia, in 2007. During his graduation, he was awarded a Gold Medal for his academic excellence from the Faculty of Science and Technology, Mekelle University.

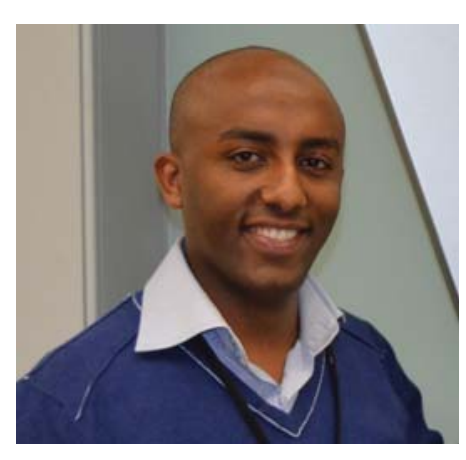

During his stay in Mekelle University as a student, he had actively participated in various extracurricular activities in different capacities. Most notably, he was the president of the Students' Council.

After his graduation, he worked as an assistant lecturer in the Department of Electrical Engineering, Mekelle University, for a year. He was also dean of Students of Mekelle University Main Campus (Endayesus) .

In 2008, he received a full Huygens Scholarship to pursue his graduate studies in the University of Twente. In 2010, he completed his MSc. in Electrical Engineering and graduated with honors (Cum Laude).

Right after, he started his PhD in Robotics and Mechatronics (formerly Control Engineering) group at the University of Twente. His PhD research, reported in this thesis, was part of a Collaborative project ITC-248669 supported by the European Community under the $7^{\text {th }}$ Framework Programme entitled "Innovative aerial service robots for remote inspections by contact (AIRobots)".

His research interests, besides tele-robotics, include robotics, mechatronics, nonlinear control, dynamic modeling and simulation of physical systems. Besides academia, his hobbies include photography, playing football and volleyball, swimming, running, table tennis, watching movies and reading biographies. 